Florida International University FIU Digital Commons

\title{
Analysis and Modeling of Advanced Power Control and Protection Requirements for Integrating Renewable Energy Sources in Smart Grid,
}

Amirhasan Moghadasiriseh

Florida International University, amogh004@fiu.edu

DOI: $10.25148 /$ etd.FIDC000255

Follow this and additional works at: https://digitalcommons.fiu.edu/etd

Part of the Electrical and Computer Engineering Commons

\section{Recommended Citation}

Moghadasiriseh, Amirhasan, "Analysis and Modeling of Advanced Power Control and Protection Requirements for Integrating Renewable Energy Sources in Smart Grid," (2016). FIU Electronic Theses and Dissertations. 2469.

https://digitalcommons.fiu.edu/etd/2469 


\section{FLORIDA INTERNATIONAL UNIVERSITY}

Miami, Florida

\section{ANALYSIS AND MODELING OF ADVANCED POWER CONTROL AND PROTECTION REQUIREMENTS FOR INTEGRATING RENEWABLE ENERGY SOURCES IN SMART GRID}

A dissertation submitted in partial fulfillment of the requirements for the degree of DOCTOR OF PHILOSOPHY in ELECTRICAL ENGINEERING by Amirhasan Moghadasiriseh 


\section{To: Interim Dean Ranu Jung}

College of Engineering and Computing

This dissertation, written by Amirhasan Moghadasiriseh, and entitled Analysis and Modeling of Advanced Power Control and Protection Requirements for Integrating Renewable Energy Sources in Smart Grid, having been approved in respect to style and intellectual content, is referred to you for judgment.

We have read this dissertation and recommend that it be approved.

Osama A. Mohammed

Nezih Pala

Sakhrat Khizroev

Walid Saad

Arif I. Sarwat, Major Professor

Date of Defense: March 29, 2016

The dissertation of Amirhasan Moghadasiriseh is approved.

Interim Dean Ranu Jung College of Engineering and Computing

Andrés G. Gil

Vice President for Research and Economic Development and Dean of the University Graduate School

Florida International University, 2016 
(C) Copyright 2016 by Amirhasan Moghadasiriseh All rights reserved. 


\section{DEDICATION}

I would like to dedicate this dissertation to my lovely wife, Niloufar, to my beloved parents, Rahmat and Ensi, and to my sister, Maryam, and my brother Alireza. The completion of this work would not have been possible without their encouragement, support, understanding, patient, and most of all love. 


\section{ACKNOWLEDGMENTS}

First, I would like to express my sincere appreciation and gratitude to my major Professor, Arif I. Sarwat, for his support, outstanding guidance, and endless encouragement during the last three years of my doctoral study. I would also like to show my gratitude to the members of my committee for their support, insightful comments, and constructive suggestions, in particular, Dr. Osama A. Mohammed, Nezih Pala, Dr. Sakhrat Khizroev, and Dr. Walid Saad.

I would like to acknowledge the partial research support provided from the National Science Foundation (NSF) throughout my years of research. I also acknowledge the doctoral DEA fellowship from FIU graduate school during the spring semester of 2016 of my studies at Florida International University. Next, I would like to thank my colleagues in Energy, Power and Sustainability Group for creating a wonderfully collaborative work environment.

Finally, I am thankful for the staff of ECE department at FIU, especially to Mrs. Pat Brammer and Mr. Oscar Silveria for their great commitment to student services. 


\author{
ABSTRACT OF THE DISSERTATION \\ ANALYSIS AND MODELING OF ADVANCED POWER CONTROL AND \\ PROTECTION REQUIREMENTS FOR INTEGRATING RENEWABLE ENERGY \\ SOURCES IN SMART GRID \\ by \\ Amirhasan Moghadasiriseh \\ Florida International University, 2016 \\ Miami, Florida \\ Professor Arif I. Sarwat, Major Professor
}

Attempts to reduce greenhouse gas emissions are promising with the recent dramatic increase of installed renewable energy sources (RES) capacity. Integration of large intermittent renewable resources affects smart grid systems in several significant ways, such as transient and voltage stability, existing protection scheme, and power leveling and energy balancing. To protect the grid from threats related to these issues, utilities impose rigorous technical requirements, more importantly, focusing on fault ride through requirements and active/reactive power responses following disturbances. This dissertation is aimed at developing and verifying the advanced and algorithmic methods for specification of protection schemes, reactive power capability and power control requirements for interconnection of the RESs to the smart grid systems.

The first findings of this dissertation verified that the integration of large RESs become more promising from the energy-saving, and downsizing perspective by introducing a resistive superconducting fault current limiter (SFCL) as a self-healing equipment. The proposed SFCL decreased the activation of the conventional control scheme for the wind power plant (WPP), such as dc braking chopper and fast pitch angle control systems, thereby increased the reliability of the system. 
A static synchronous compensator (STATCOM) has been proposed to assist with the uninterrupted operation of the doubly-fed induction generators (DFIGs)-based WTs during grid disturbances. The key motivation of this study was to design a new computational intelligence technique based on a multi-objective optimization problem (MOP), for the online coordinated reactive power control between the DFIG and the STATCOM in order to improve the low voltage ride-through (LVRT) capability of the WT during the fault, and to smooth low-frequency oscillations of the active power during the recovery.

Furthermore, the application of a three-phase single-stage module-integrated converter (MIC) incorporated into a grid-tied photovoltaic (PV) system was investigated in this dissertation. A new current control scheme based on multivariable PI controller, with a faster dynamic and superior axis decoupling capability compared with the conventional PI control method, was developed and experimentally evaluated for three-phase PV MIC system.

Finally, a study was conducted based on the framework of stochastic game theory to enable a power system to dynamically survive concurrent severe multi-failure events, before such failures turn into a full blown cascading failure. This effort provides reliable strategies in the form of insightful guidelines on how to deploy limited budgets for protecting critical components of the smart grid systems. 


\section{TABLE OF CONTENTS}

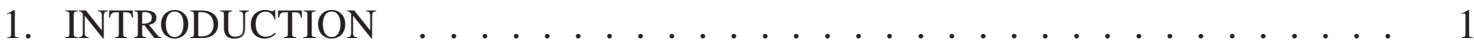

1.1 General Statement of Problem Area . . . . . . . . . . . . . . . . . . . 1

1.2 Motivation and Purpose of Research . . . . . . . . . . . . . . . . 11

1.3 Literature Review . . . . . . . . . . . . . . . . . . . . . . . . . . . 12

1.4 Research Objectives and Original Contributions . . . . . . . . . . . . 20

1.5 Dissertation Organization . . . . . . . . . . . . . . . . . . . . . 23

2. A COMPREHENSIVE INVESTIGATION OF LVRT IMPROVEMENT METHODS FOR FIXED-SPEED WIND POWER GENERATORS . . . . . . . . . . 26

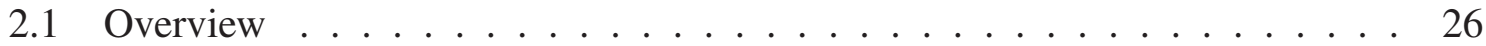

2.2 FSIG-based wind turbine $\ldots \ldots \ldots \ldots$

2.2 .1 Steady-state operation . . . . . . . . . . . . . . . . . . 27

2.2.2 Transient-state operation . . . . . . . . . . . . . . . . . . . . . . . . . . 29

2.3 Review of series-connected solutions . . . . . . . . . . . . . . . . 32

2.3.1 Thyristor-controlled series compensation (TCSC) . . . . . . . . . . . . 32

2.3.2 Dynamic voltage restorer (DVR) . . . . . . . . . . . . . . . . . . 34

2.3.3 Series dynamic braking resistor (SDBR) . . . . . . . . . . . . 36

2.3.4 Magnetic energy recovery switch (MERS) . . . . . . . . . . . . 38

2.3.5 Fault current limiter (FCL) . . . . . . . . . . . . . . . . . . . 40

2.4 Review on shunt-connected solutions . . . . . . . . . . . . . . . . . . . 44

2.4.1 Static var compensator $(\mathrm{SVC}) \ldots \ldots \ldots$. . . . . . . . . . . . 44

2.4.2 Static synchronous compensator (STATCOM) . . . . . . . . . . . . 46

2.4.3 Superconducting dynamic synchronous condenser (SDSC) . . . . . . . . 48

2.5 Review on hybrid-connected solutions . . . . . . . . . . . . . . . . 49

2.6 Technical-economical evaluation study of the LVRT methods . . . . . . . . 51

2.6.1 Technical comparative study . . . . . . . . . . . . . . . . . . . 51

2.6 .2 Economical comparative study . . . . . . . . . . . . . . . . . . 54

2.7 Simulation results and performances comparison $\ldots \ldots \ldots \ldots$

2.8 Summary . . . . . . . . . . . . . . . . . . . 63

3. APPLICATION OF SUPERCONDUCTIVITY DEVICES ON WIND POWER

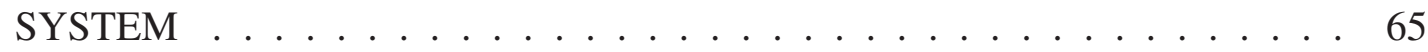

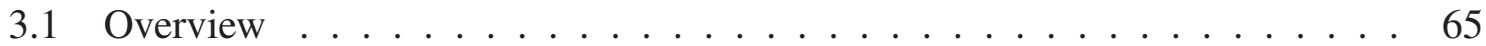

3.2 LVRT Improvement of FSIG WTs Using UPQC and SFCL . . . . . . . . 65

3.2.1 FSIG-Based Wind turbine . . . . . . . . . . . . . . . . . . 65

3.2.2 Proposed UPQC System … . . . . . . . . . . . . . . . . . . . . 67

3.2.3 Electro-Thermal Modeling of a Resistive SFCL . . . . . . . . . . . . . . 68

3.2.4 Numerical Simulation Analysis . . . . . . . . . . . . . . . . . . . 70

3.3 LVRT Improvement of PMSG WTs Using a Resistive SFCL . . . . . . . 74

3.3.1 Modeling of the PMSG-Based Wind Turbine . . . . . . . . . . . 76 
3.3.2 Power Control Strategy . . . . . . . . . . . . . . . . . . . . . . . . . . 80

3.3.3 Electro-Thermal Modeling of a RSFCL . . . . . . . . . . . . . . . 82

3.3.4 Numerical Simulation Analysis . . . . . . . . . . . . . . . . . . . . 84

3.3.5 Optimal Scheme Performance . . . . . . . . . . . . . . . . . . . . . 92

3.4 Power Management of DC grid Involving PMSG WTs and SMES _. . . . . 98

3.4.1 Motivation and related works . . . . . . . . . . . . . . . . . . . 98

3.4 .2 Overall System Configuration . . . . . . . . . . . . . . . . . . . . . . . 99

3.4 .3 SMES System Description . . . . . . . . . . . . . . . . . . . 100

3.4.4 Control Scheme in Standalone Mode . . . . . . . . . . . . . . . . . . 103

3.4.5 Simulation Results and Discussion . . . . . . . . . . . . . . . . 108

3.5 Economic and Practical Feasibility of the RSFCL . . . . . . . . . . . . 112

3.6 Summary . . . . . . . . . . . . . . . . . . . . . . . . 115

4. IMPROVING OPERATIONAL RESILIENCE OF RENEWABLE ENERGY SYS-

TEM USING A STATCOM . . . . . . . . . . . . . . . . . . . . 117

4.1 Overview . . . . . . . . . . . . . . . . . . . . . . 117

4.2 DFIG Wind Farm Model . . . . . . . . . . . . . . . . . . . . 118

4.2 .1 Wind Turbine Model . . . . . . . . . . . . . . . . . . . . . . . 118

4.2.2 Drive Train Model . . . . . . . . . . . . . . . . . . . . . . . . . . . . . . 119

4.2 .3 Generator Model . . . . . . . . . . . . . . . . . . . . . . . . . . . . . . 119

4.3 Description of the Control Systems . . . . . . . . . . . . . . . . . . 120

4.3.1 Rotor side Converter (RSC) Controller . . . . . . . . . . . . . . . . . . 121

4.3.2 Grid side Converter (GSC) Controller . . . . . . . . . . . . . . . . 122

4.3.3 STATCOM Controller . . . . . . . . . . . . . . . . . . . . . . . 123

4.4 Coordinated Reactive Power Control . . . . . . . . . . . . . . . . . . . . 123

4.4 .1 MOP Model Formulation . . . . . . . . . . . . . . . . . . . . . . 125

4.4 .2 Solution Method . . . . . . . . . . . . . . . . . . . . . . 128

4.4.3 Tuning the FLC Model Using NSA . . . . . . . . . . . . . . . . . . . 129

4.5 Computer Simulation Study _ . . . . . . . . . . . . . . . . . . 136

4.5.1 Power System Case Study ． . . . . . . . . . . . . . . . . . 136

4.5.2 Validation of the NSA Algorithm . . . . . . . . . . . . . . 137

4.5.3 Simulation Case Studies . . . . . . . . . . . . . . . . . . . . . . . . 141

4.6 Summary . . . . . . . . . . . . . . . . . . . . . . . . . . . . . . . 144

5. ACTIVE AND REACTIVE POWER CONTROL OF THREE-PHASE SINGLE-

STAGE GRID-TIED PV MODULE-INTEGRATED CONVERTER . . . . . . 146

5.1 Overview . . . . . . . . . . . . . . . . . . . . 146

5.2 Configuration and Modulation Strategy _. . . . . . . . . . . . . . 147

5.3 State-Space Average Modeling of Grid-Tied CSI-Based MIC . . . . . . . . . 152

$5.3 .1 \quad$ Large-Signal Model . . . . . . . . . . . . . . . . . . . . 153

5.3.2 Small-Signal Model . . . . . . . . . . . . . . . . . . . . . . . 157

5.3.3 Open-Loop Stability Analysis . . . . . . . . . . . . . . . . . . . . . 159

5.4 Power Control System for Three-Phase CSI-Based MIC . . . . . . . . . . . 162 
5.4.1 Comparative Study of Conventional and Multivariable PI-Based $d q$ Current Control . . . . . . . . . . . . . . . . . . 163

5.4.2 Active and Reactive Power Control Implementation . . . . . . . . . . . 165

5.5 Simulation and Experimental Verification . . . . . . . . . . . . . . 168

5.5.1 Simulation Results . . . . . . . . . . . . . . . . . . . . . . . . . . . . . 169

5.5.2 Experimental Results . . . . . . . . . . . . . . . . . . . . . . . 173

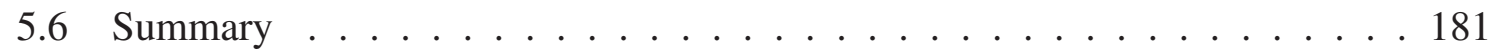

6. EXPLORING RELIABLE STRATEGIES FOR PROTECTING SMART GRID UNDER MULTI-FAILURE EVENTS . . . . . . . . . . . . . . . . . 183

6.1 Overview . . . . . . . . . . . . . . . . . . . 183

6.2 System Model and Problem Formulation . . . . . . . . . . . . . . . . . 184

6.3 Stochastic Game-theoretic Approach . . . . . . . . . . . . . . . . . . . . 187

$6.3 .1 \quad$ Nash Equilibrium Strategies _ . . . . . . . . . . . . . . . . . 188

6.4 Game Solution . . . . . . . . . . . . . . . . . . . . . . . . . . 191

6.5 Simulation Results and Analysis . . . . . . . . . . . . . . . . . . . . 193

6.6 Summary . . . . . . . . . . . . . . . . . . . . . . . 202

7. CONCLUSION AND FUTURE WORK . . . . . . . . . . . . . 205

7.1 Conclusion . . . . . . . . . . . . . . . . . . . . . . 205

7.2 Future Work . . . . . . . . . . . . . . . . . . . . . . . . . . . . 209

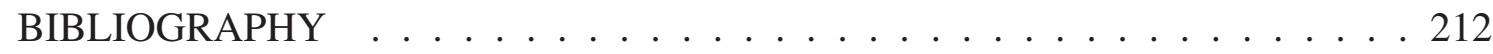

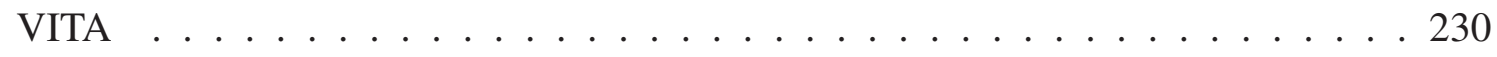




\section{LIST OF TABLES}

TABLE

PAGE

2.1 Technical comparison of LVRT improvement methods for FSIG-based WTs. 52

$2.2 \quad$ FSIG-based WT parameters. . . . . . . . . . . . . . . 60

2.3 Synchronous generator parameters. . . . . . . . . . . . 60

2.4 Parameters of FACTS devices. . . . . . . . . . . . . . . . 60

3.1 Characteristics of the wind farm induction generator. . . . . . . . . 71

3.2 Parameters of SFCL. . . . . . . . . . . . . . . 71

3.3 Characteristics of the wind farm induction generator. . . . . . . . . . 86

3.4 Parameters of SFCL . . . . . . . . . . . . . . . 86

3.5 Limits of Variables for Optimization Problem. . . . . . . . . . . . . . . . 94

3.6 Achieved Optimal Alternatives Using AHP Method. . . . . . . . . . . . . 98

3.7 Optimization Results of The SMES Coil. . . . . . . . . . . . . . . 109

4.1 Normalized Simulated Annealing (NSA) Algorithm . . . . . . . . . . . 130

4.2 Parameters Used to Generate Initial Sigmoid Membership Functions for Input and Output Variables . . . . . . . . . . . . . . . . 132

4.3 Parameters Used to Generate Tunable Sigmoid Membership Functions for Output Variables ... . . . . . . . . . . . . 132

4.4 Fuzzy Rules Generated by NSA . . . . . . . . . . . . . . . . . 134

4.5 Parameters of the $1.5-\mathrm{MW}$ DFIG . . . . . . . . . . . 137

4.6 Pareto Optimality Results Using NSA Algorithm. . . . . . . . . . . . . 138

4.7 Comparison of the NSA Performance with NSGA-II . . . . . . . . . . . 140

$5.1 \quad$ System Parameters . . . . . . . . . . . . . . . . 159

6.1 Shed Load due to Failures in the IEEE 14-Bus System. . . . . . . . . . . . . 195

6.2 Shed Load due to Failures in the IEEE 39-Bus System . . . . . . . . . . . 200

6.3 Nash Equilibrium Strategies of Operator for Various Budgets . . . . . . . . 200

6.4 Nash Equilibrium Strategies of Operator for Various Budgets. . . . . . . . . 201 


\section{LIST OF FIGURES}

FIGURE

PAGE

1.1 Danish grid codes. (a) LVRT requirement. (b) Reactive power support re-

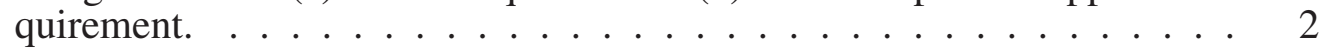

1.2 Different wind generator technologies. . . . . . . . . . . . . 4

1.3 Grid-connected PV system architectures. (a) Centralized inverter. (b) Multistring inverter. (c) AC module-integrated converter (MIC) or also called micro-inverter. ................... 7

1.4 Resistive superconducting fault current limiter [22]. . . . . . . . . . . . 9

1.5 An illustrative example of the smart grid with RES and its abstracted undirected graph, where red stars represent several failure scenarios occur in the smart grid system. . . . . . . . . . . . . . . . . 10

1.6 Four options for connecting PV MIC systems. (a) MIC with line-frequency transformer. (b) MIC with high-frequency transformer. (c) Single-stage z-source MIC. (d) Single-stage current source MIC. . . . . . . . . . . .

2.1 The main components of the wind turbine based on FSIG. . . . . . . . . . 27

2.2 The output power of a wind turbine as a function of the wind speed. . . . . . 27

2.3 Conventional pitch angle control used in FSIG-based wind turbine. . . . . . 28

2.4 Modified pitch angle control used in FSIG-based wind turbine. . . . . . . . . 31

2.5 Classified LVRT capability enhancement methods. . . . . . . . . . . . . 32

2.6 TCSC module installed outside the wind farm with the basic control scheme. 33

2.7 Principle operation of the dynamic voltage restorer and power flow during the voltage dip compensation. . . . . . . . . . . . 34

2.8 Single-phase vector diagram. (a) Voltage dip compensation with DVR. (b) Voltage dip compensation once the phasor has been rotated. . . . . . . . 35

2.9 Various types of SDBR; (a) Single-stage scheme. (b) Two-stage switching scheme. (c) Variable resistor scheme using soft-starter. . . . . . . . . . 37

2.10 Single-phase vector diagram for voltage dip compensation with SDBR. . . . 38

2.11 Circuit configuration of the MERS for controlling the series-injected voltage. 38

2.12 Switching patterns for one current cycle. (Left part) voltage and current waveform of MERS; (Right part) the flow of the current through the MERS for the different areas illustrated on the left. . . . . . . . . . . 39 
2.13 Fault current limiter topology. (a) Bridge-type FCL (BFCL). (b) Modified configuration of BFCL. . . . . . . . . . . . . . 40

2.14 Modified configuration of BFCL installed outside the wind farm with the control scheme. . . . . . . . . . . . . . . . . . . . 42

2.15 Operation of resistive SFCL installed in transmission line including fault current and voltage profile at the wind turbine terminal.

2.16 Shunt compensation system for wind driven induction generator using SVC along with the basic control system. . . . . . . . . . . . . . . 45

2.17 The structure of the FSIG-based WT along with STATCOM connected to wind turbine terminal. . . . . . . . . . . . . . . . 46

2.18 The HTS DSC Concept (a) Structure of the SDSC. (a) Var curve for conventional synchronous and HTS DSC machines. . . . . . . . . . . . 48

2.19 The structure of the proposed system: FSIG-based wind turbine with UPQC and SFCL connected to the grid. . . . . . . . . . . . . . . . . 49

2.20 The structure of the proposed system: FSIG-based wind turbine with current source UPQC and SFCL connected to the dc link. . . . . . . . . . . .

2.21 Configuration of unified compensation system (UCS) connected to wind tur-

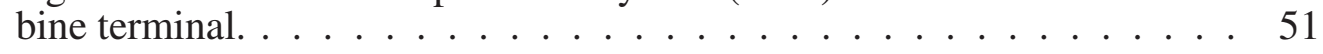

2.22 Installed cost of wind power projects in three area; 2010, 2011, and 2015. . . 55

2.23 An operating cost comparison between FACTS devices. (a) SVC, TCSC, and UPFC. (b) SVC and STATCOM. . . . . . . . . . . . . . 56

2.24 Average and estimated overall cost of LVRT solutions for operating range of around 100 MVar. . . . . . . . . . . . . . . . 58

2.25 Single-line diagram of the power network including a series-connected or shunt-connected compensator. . . . . . . . . . . . . . . . . 59

2.26 FSIG terminal voltage at PCC . . . . . . . . . . . . . 61

2.27 FSIG active power output at PCC . . . . . . . . . . . . . 61

2.28 Reactive power absorbed by FSIG. . . . . . . . . . . . . . . . 62

2.29 Stator current of the FSIG. . . . . . . . . . . . . . . . . . . . . 63

2.30 FSIG rotor speed. . . . . . . . . . . . . . . . . . . . 63

3.1 Structure of the proposed system: FSIG-based wind turbine with UPQC and SFCL connected to the grid. . . . . . . . . . . . . . . 66 
3.2 Pitch angle Control used in FSIG-based wind turbine. . . . . . . . . . . . 67

3.3 Proposed control structure of the UPQC to compensate voltage sag and current harmonic. ........................ 69

3.4 Current harmonics compensation. (a) Load and grid current. (b) Shunt active

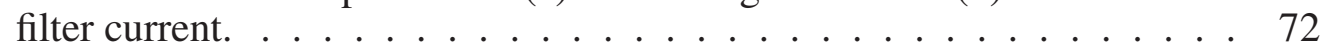

3.5 Voltage sags compensation without SFCL. (a) Grid voltage and series APF voltage. (b) PCC voltage with compensation. . . . . . . . . . . . 72

3.6 Performance evaluation of the SFCL model. (a) Fault current without and with SFCL in a single-phase system. (b) Resistance variation and temperature rise. . . . . . . . . . . . . 73

3.7 Voltage-dip characteristic at the generator terminal. . . . . . . . . . . 74

3.8 Voltage sags compensation with SFCL. (a) Grid voltage and series APF voltage. (b) PCC voltage with compensation. . . . . . . . . . . . . . 74

3.9 Active and reactive power delivered to the grid at PCC . . . . . . . . 75

3.10 Proposed combinatorial PMSG-based WPP and RSFCL. . . . . . . . . . 76

3.11 Subsystems model used in PMSG-based wind turbine. . . . . . . . . . . . 77

3.12 Wind turbine characteristics. . . . . . . . . . . . . 77

3.13 Conventional pitch angle control used in FSIG-based wind turbine. . . . . . 78

3.14 Proposed power control strategy for WPP. (a) Control block diagram of the generator-side VSC. (b) Control block diagram of the grid-side VSC. . . 80

3.15 Equivalent circuit of proposed system in fault condition. . . . . . . . . . 83

3.16 Combinatorial PMSG-based WT and RSFCL model using SimPower Systems. 85

3.17 RSFCL model response. (a) Fault current waveform without and with RSFCL in a single-phase system. (b) Resistance variation in flux flow and normal state. (c) Temperature rise. . . . . . . . . . . . . .

3.18 Operation of the proposed combinatorial WPP and RSFCL during and after fault (a) Voltage profile at wind farm terminal (b) dc-link voltage with and without RSFCL. (c) Active and reactive power at the PCC without the RSFCL. (d) Active and reactive power at the PCC with the RSFCL. .

3.19 Operational setting for proposed system. (a) Wind speed. (b) Wind Direction. (c) Nacelle yaw control performance. . . . . . . . . . . . . . . 89

3.20 Power delivered to the grid at PCC with and without RSFCL. (a) Active power output. (b) Reactive power output. . . . . . . . . . . . . . . 90 
3.21 Dynamic performance of the turbine side with and without applying the RS-

FCL. (a) Rotational turbine speed. (b) Aerodynamic rotor torque. . . . . 91

3.22 Dynamic performance of the generator side with and without applying the

RSFCL. (a) Rotational generator speed. (b) Generator torque. . . . . . . 92

3.23 Hierarchy process for optimal combinatory PMSG-based WT and RSFCL. . 95

3.24 Multi-objective optimization using AHP, Pareto front for three criteria. . . 97

3.25 Combination of PMSG- based on VSWT and SMES system in de microgrid. 99

3.26 Estimated energy of the SMES according to lower and upper coil current limits.101

3.27 Cross section of multi-layer solenoid coil. . . . . . . . . . . . . . . . 102

3.28 Control diagram of PMSG-based variable speed wind turbine. . . . . . . . . 104

3.29 Generator side controller of PMSG. . . . . . . . . . . . . . . . . . . . 104

3.30 Control diagram of dc-de converter with SMES coil. . . . . . . . . . . . 106

3.31 Energy management strategies for the standalone DC Microgrid. . . . . . . . 107

3.32 Progress of the solution of the proposed optimization considering $V_{\text {coil }}$ as an objective function. . . . . . . . . . . . . . . . . . . . 109

3.33 System operation during normal charging/discharging mode (Case 1). (a) Wind speed. (b) SMES coil current. (c) Power generated by the wind turbine and total load. (d) DC-link voltage. . . . . . . . . . . . . . 110

3.34 System operation during prolonged low-wind mode (Case 2). (a) Wind speed. (b) SMES coil current. (c) Power generated by the wind turbine and total load. (d) DC-link voltage. . . . . . . . . . . . . . . . . 113

3.35 HTS wire cost. (a) Dollar per Kilo amp per Meter. (b) Dollar per Meter. . . . 114

4.1 Wind turbine characteristics. . . . . . . . . . . . . . . . . 118

4.2 Overall control scheme of the proposed system. $v_{d r 2}=-s \omega_{s} \sigma L_{r} i_{q r} ; v_{q r 2}=$

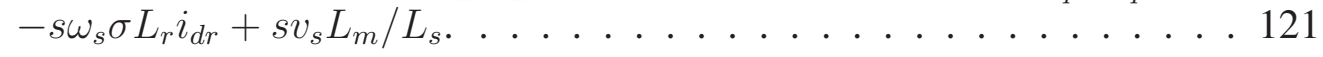

4.3 Overall block diagram of the proposed PCRPC for online application. . . . . 124

4.4 Fuzzy membership functions. (a) Fuzzy input subsets of the $T f_{1}$ and $T f_{2}$.

(b) Fuzzy output subset of the $Q_{R S C}^{*}$. (c) Fuzzy output subset of the $Q_{S T A T}^{*} \cdot 131$

4.5 Single-line diagram of the multi-machine benchmark power system including a wind turbine and a STATCOM connected to the PCC. . . . . . . 137 
4.6 Three dimensional representations of control variables versus objective function. . . . . . . . . . . . . . . . . . 138

4.7 NSA-based optimization progress of the reactive power commands (control variables) and two objectives for the case 3; x-axis denotes to the number of iteration.

4.8 Pareto front that was obtained with NSGA-II and NSA algorithm. . . . . . . 141

4.9 Dynamic response of the 12-bus system with and without proposed control system considering case 1 , case 3 , and case 6. (a) Voltage at PCC. (b) Output active power at the PCC. (c) Output reactive power of WT. (d) Output reactive power of STATCOM. . . . . . . . . . . . 142

5.1 Three-phase single-stage grid-tied CSI test system. . . . . . . . . . . . 148

5.2 Six sections in line-to-line voltage phasors. . . . . . . . . . . . . . 148

5.3 Switching sequence of all six sectors. . . . . . . . . . . . . . 149

5.4 Three intervals for proposed switching patterns for Sector I. . . . . . . . . . 150

5.5 Voltage and current of the dc-link inductor over one $T_{s}$ in a CSI, while $V_{1}$ and $V_{2}$ are line-line voltage output. . . . . . . . . . 151

5.6 Variations of boost ratio $\beta$ versus modulation index $m_{i}$ and the modulation angle $\alpha_{0}$ (degree) for Sector I. . . . . . . . . . . . . . 153

5.7 Circuit diagrams and state equation of the three-phase grid-tied CSI-based MIC for Sector I. (a) Charging state, $S_{1}$ and $S_{4}$ on. (b) Discharging state, $S_{1}$ and $S_{6}$ on. (c) Discharging state, $S_{1}$ and $S_{2}$ on. . . . . . . . 154

5.8 CSI-based MIC equivalent circuits. (a) dc-side equivalent circuit. (b) $d$-axis equivalent circuit. (c) $q$-axis equivalent circuit. . . . . . . . . . . . . . . 158

5.9 Location of eigenvalues of small-signal model. (a) $L_{d c}$ varies from $5 \mathrm{mH}$ to $20 \mathrm{mH}$. (b) $C_{f}$ varies from $5 \mu \mathrm{F}$ to $30 \mu \mathrm{F}$. (c) $L_{f}$ varies from $0.5 \mathrm{mH}$ to $2.5 \mathrm{mH}$. (d) $R_{f}$ varies from $2 \Omega$ to $5 \Omega$. (e) $m_{i}$ varies from 0.2 to 1 . (e) $\alpha_{0}$ varies from $-\pi / 6$ to $11 \pi / 6$.

5.10 Structural diagram of $d q$ current control. (a) Conventional PI-based control. (b) Multivariable PI-based control with voltage feedforward. . . . . . . . 164

5.11 Overall control block diagram of the three-phase grid-tied CSI-based MIC system. . . . . . . . . . . . . . . . . 166

5.12 Layout of experimental setup. . . . . . . . . . . . . . 168

5.13 Performance of the proposed method in tracking active and reactive power changes for scenario I. (a) active power step-up. (b) reactive power stepdown. (c) dc-link current. (d) voltage and current waveforms. . . . . . 170 
5.14 Performance of the proposed method in tracking active and reactive power changes for scenario II. (a) active power step-up. (b) reactive power stepdown. (c) dc-link current. (d) voltage and current waveforms. . . . . . 172

5.15 Experimental performance of the proposed method in tracking active and reactive power changes for scenario I. (a) active power step-down. (b) reactive power step-up. (C) dc-link current. (d) voltage and current waveforms. . . . . . . . . . . . . . . . . . . . 174

5.16 Experimental performance of the proposed method in tracking active and reactive power changes for scenario II. (a) active power step-up. (b) reactive power step-down. (C) dc-link current. (d) voltage and current waveforms. . . . . . . . . . . . . . 175

5.17 Experimental results for the transient response during step changes in active power command $P_{C S I}^{*}$. (a) Conventional PI-based controller. (b) Multivariable PI-based controller. . . . . . . . . . . . . . . . 176

5.18 Experimental results for the transient response of multivariable PI controller during step changes in active power command. Description of plots: modulation index $m_{i}$ on top and modulation angle $\alpha_{0}$ on bottom. . . . 177

5.19 Experimental results for the transient response of multivariable PI controller during step changes in active power command. Description of plots: phase-a waveform of the grid voltage on top and grid current on bottom.

5.20 Experimental results for the transient response of dc-link current during step changes in active power command. . . . . . . . . . . 178

5.21 Experimental results for the transient response during step changes in reactive power command $Q_{C S I}^{*}$. (a) Conventional PI-based controller. (b) Multivariable PI-based controller. . . . . . . . . . . . . . . . . . . 179

5.22 Experimental results for the transient response of multivariable PI controller during step changes in reactive power command. Description of plots: modulation index $m_{i}$ on top and modulation angle $\alpha_{0}$ on bottom. . . . 180

5.23 Experimental results for the transient response of multivariable PI controller during step changes in reactive power command. Description of plots: phase-a waveform of the grid voltage on top and grid current on bottom.

5.24 Experimental results for the transient response of dc-link current during step changes in reactive power command. . . . . . . . . . 181

6.1 Flowchart of proposed stochastic game approach. . . . . . . . . . . . 193

6.2 The IEEE 14-bus system contains 2 generators, 20 transmission lines. (a) Bus diagram. (b) Link Diagram. . . . . . . . . . . . . . . . . . 194 
6.3 Nash equilibrium strategies with various discounted factors $\beta$ for IEEE 14bus system. (a) strategies of generating multi-failure events in state $s_{1}$. (b) operator's response strategies in state $s_{1}$. (c) strategies of generating severe multi-failure events in state $s_{2}$. (d) operator's response strategies in state $s_{2} \ldots \ldots \ldots \ldots 6 . \ldots \ldots \ldots$

6.4 The IEEE 39-bus system contains 10 generators, 34 transmission lines, and 18 loads. . . . . . . . . . . . . . . . . . . 198

6.5 Nash equilibrium strategies with various discounted factors $\beta$ for the IEEE 39-bus system. (a) severe generated multi-failure event strategies in state $s_{1}$. (b) operator's response strategies in state $s_{1}$. (c) severe generated multi-failure events strategies in state $s_{2}$. (d) operator's response strate-

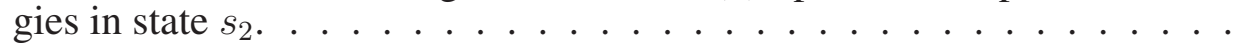

6.6 Comparison of expected cost of load shedding in state $s_{1}$ of the stochastic game, (a) IEEE 14-bus system. (b) IEEE 39-bus system. . . . . . . . . . 203 


\section{LIST OF ACRONYMS}

FSIG

PMSG

DFIG

RSC

GSC

LVRT

RES

MIC

SFCL

HTS

LTS

SMES

ESS

FACTS

FLC

BC

STATCOM

PSO

BFA

GA

ADP

HDP

VSI

CSI

PPWM
Fixed-speed induction generator

Permanent magnet synchronous generator

doubly fed induction generator

Rotor-side converter

Grid-side converter

Low voltage ride-through

Renewable energy sources

Module-integrated converter

Superconducting fault current limiter

High temperature superconducting

Low temperature superconducting

Superconducting magnetic energy storage

Energy storage system

Flexible AC transmission system

Fuzzy logic controller

Braking chopper

Static synchronous compensators

Particle swarm optimization

Bacterial foraging algorithm

Genetic algorithm

Adaptive dynamic programming

Heuristic dynamic programming

Voltage source inverter

Current source inverter

Phasor pulse-width-modulated (PPWM) 
IGBT

PI

THD

MOP

NSA

TCSC

DVR

UPQC

SVC

MERS

UCS

EPRI

VSWT

AHP

TSR

MPPT

PLL

MCDM

PCS

TPSI

MDP

DOE

NERC

HILF

DWADP
Isolated Gate Bipolar Transistor

ProportionalIntegral

Total Harmonic Distortion

Multi-objective optimization problem

Normalized simulated annealing

Thyristor-controlled series compensation

Dynamic voltage restore

Unified power quality conditioner

Static var compensator

Magnetic energy recovery switch

Unified compensation system

Electric power research institute

Variable speed wind turbine

Analytic hierarchy process

Tip speed ratio

Maximum power point tracking

Phase-locked loop

Multi-criteria decision making

Power conditioning system

Transient power severity index

Markov decision processes

Department of energy

North american electric reliability corporation

High-impact, low-frequency

Distributed wide area differential protection 


\section{CHAPTER 1}

\section{INTRODUCTION}

This introductory chapter contains five sections. The first section describes the background of the problem. The second section introduces motivations and research purposes of the dissertation. The third section articulates objectives and contributions of this research. The summary of the literature search of the problem is presented in fourth section. Finally, the fifth section presents the general organization of this dissertation.

\subsection{General Statement of Problem Area}

It has been very clear from recent studies and documentations that the fossil fuels would last only a few more decades. The cost of fossil fuel has become a major challenge for all of human kind. Not only the economic value but the environmental impacts of fossil fuels have clearly made us move toward alternatives [1]. The greatest alternatives that can really make a difference for sustainability, such as reducing greenhouse gases and long term economics, are the renewable energy sources (RES) like wind and solar power.

Integration of the large RESs affects the network in several significant ways, such as transient and voltage stability, short circuit ratings, existing protection schemes, power leveling and energy balancing, and power quality [2]. To protect the network from threats related to these issues, utilities and other electric energy entities imposed rigorous technical requirements and grid code regulations, which are classified into four major categories: (i) fault ride through requirements, (ii) active and reactive power responses following disturbances, (iii) active power control or frequency regulation support and (iv) reactive power control or voltage regulation capability $[3,4]$.

RESs were traditionally tripped with circuit breakers once the voltage at their terminals reduced below $80 \%$ because the penetration level of wind power was extremely lower compared with the conventional generation systems and their impact on the grid 


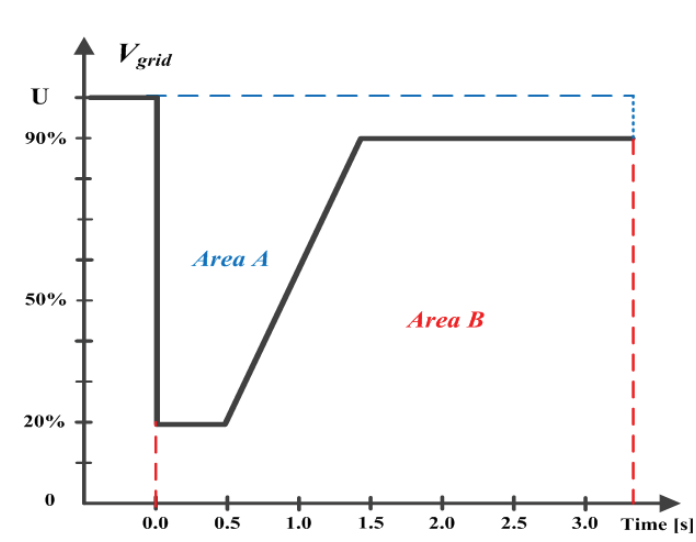

(a)

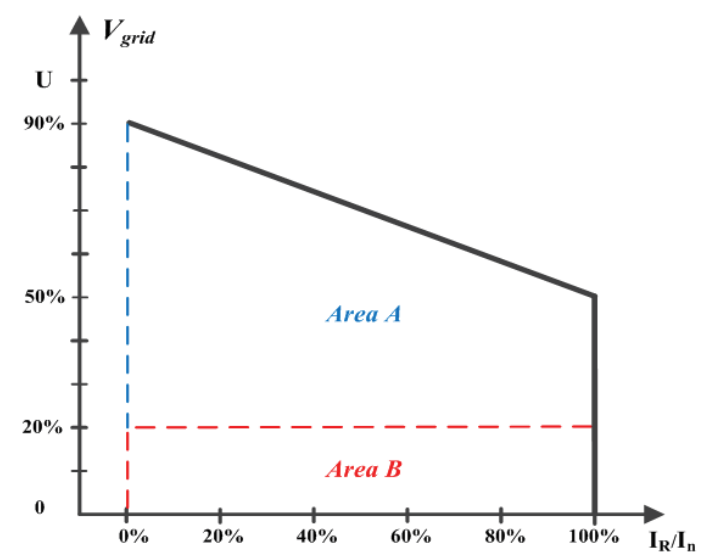

(b)

Figure 1.1: Danish grid codes. (a) LVRT requirement. (b) Reactive power support requirement.

was low. The trend toward the integration of more RES plants contributes to the increase in fault current levels, as well as voltage reductions at the terminals of RES resources, which lead to disconnection a large RES and consequently serious effects on the power system operation. Thus, new grid code requirements concerning the grid voltage support, which is called as low voltage ride-through (LVRT) capability, are regularly being developed [1-4]. This LVRT requirement implies that wind and solar generation facilities need to stay connected through a fault scenario to provide active and reactive power support to the distribution or transmission network.

Figure 1.1(a) shows a practical example of the LVRT curve defined by the Danish system operator for wind turbines (WTs) connected to the grid [5]. Based on this regulation, if the voltage remains at the level greater than $20 \%$ of nominal for a period less than 0.5 s, the WT should be connected to the grid. WTs are only stipulated to disconnect from the grid when the voltage profile falls into the Area B. Besides the LVRT requirements, some grid codes require large WTs to contribute to the voltage restoration of the power system by injecting the reactive power during the fault and the recovery period [3], while maintaining the operating point above the area of Figure 1.1(b). 
Furthermore, many power system operators in Europe and other regions of the world enforce strict rules on the active and reactive power responses of large wind farms, which are connected to the weak grids, during and even after faults in the network $[3,4]$. These strict rules are set to secure the voltage and frequency system stability following various types of disturbances. Indeed, regulations on the active power response of wind farms assist the grid to dampen the low-frequency oscillations of the grid, while the reactive power provided by wind farms can improve the voltage stability of the grid. According to the active/reactive power responses of WTs for regional grid codes discussed in [3], there are various possible conditions when the WTs are forced to meet both active and reactive current requirements. In some regional grid codes, such as in Australia, Denmark and Germany, the reactive current component has higher priority under fault conditions to guarantee voltage support; hence it is allowed to reduce the active current injection during the fault. While grid codes of Ireland, Spain and the UK devote more portion of the current to the active power component under fault conditions to guarantee frequency support; thus it is allowed to retain the active power generation during the fault in proportion to the remnant voltage. Therefore, the active and the reactive current components must be prioritized in proportion to each other based on the specific regional grid code, keeping the output current of the wind generator within the design limits [3].

\section{Wind Power Generation}

Wind turbines with the grid-connected mode of the operation play a significant role toward in sustainable energy development in the future. In 2007, 20,000 MW wind power was installed all over the world, bringing world-wide installed capacity to 94,112 MW. This is an increase of $31 \%$ compared with the 2006 market, and represents an overall increase in the global installed capacity of about $27 \%$ [6]. The present target is to achieve $12 \%$ generation of the world's electricity from wind power by 2020 . The ability of WTs to meet the international grid codes is greatly influenced by the technology used in the wind 


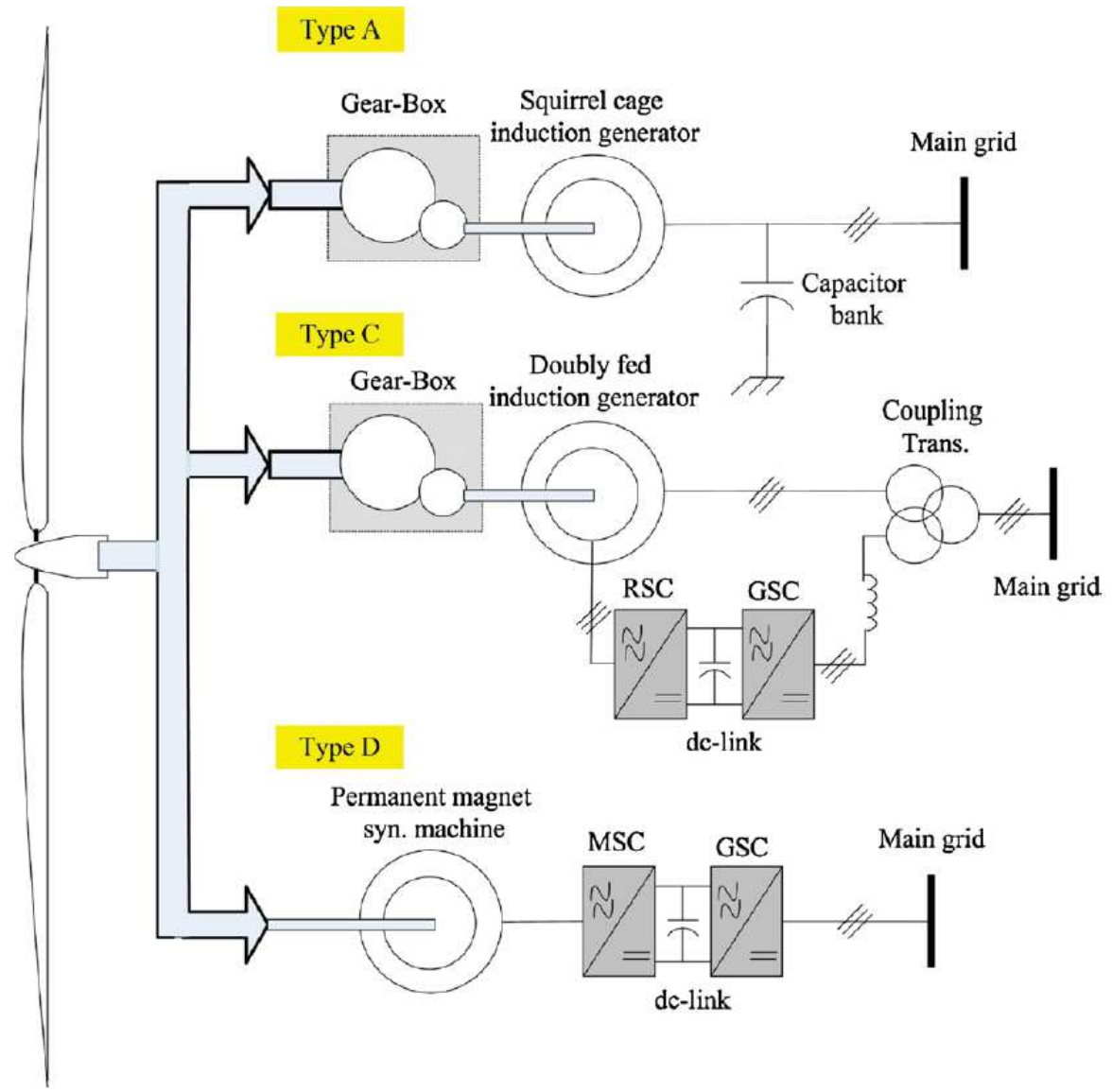

Figure 1.2: Different wind generator technologies.

farm. Figure 1.2 shows three topologies that have been widely used in wind generation systems [7].

The most conventional wind farms are based on a fixed-speed induction generator (FSIG)-type directly connected to the grid, which is considered as the simplest and most cost-effective technology among various wind generators $[3,8,9]$. Since this generator type draws the reactive power during the fault period and even after fault clearance; it not only can meet the grid code requirements, but also it exacerbates the voltage sag condition by absorbing reactive power. In order to improve LVRT capability of the FSIGbased wind turbine, several methods have already been deployed. The comprehensive review of LVRT capability improvement of this type of the wind germination has been accomplished in chapter 2 . 
Permanent magnet synchronous generators (PMSGs) with a full-rating converter offers a number of advantages for WTs, including low maintenance requirements, more reactive power supply and better ride-through capability because power electronic converters are decoupled completely from the grid $[10,11]$. However, there is a strong interaction between the turbine control system and the mechanical loads that the turbine experiences. The mechanical loads are divided into two distinct types: extreme and fatigue loads. Extreme loads are loads that a given component needs to be able to withstand once; while fatigue loads are accumulating over time and threaten to damage the turbine after several years of operation. An important source of extreme loads may occur during fault events. At the beginning of the fault, the maximum power injected into the grid reduces proportional the voltage sag amplitude, while power injected from the wind generator remains relatively constant. Due to unbalance power between the mechanical-input power and the electrical-output power, the dc-link voltage, as well as rotor speed exceeds their safety limits, which can potentially force the WT to disconnect from the grid. The quickly growing power ratings of the WTs simply exacerbate these problems. Thus, PMSG suffers from large variations of the dc-link voltage during and after the grid fault and extreme loads occurring either prior or during the shut-down process $[3,12]$. Any control system that helps to avoid unnecessary faults or that improves the behavior during the shut-down process will reduce turbine loads or contribute to turbine availability.

Recently, the majority of the WTs in power systems have been equipped with doubly fed induction generators (DFIGs) [13-16]. This technology offers the advantages of the variable speed operation with active and reactive powers control using both the rotor-side converter (RSC) and grid-side converter (GSC), which are rated for only a small fraction (25\% $\sim 30 \%$ ) of the rated power generators. One of the technical problems related to the DFIG-based WTs is the grid disturbances or low voltage ride-through (LVRT) capability. This sudden grid voltage reduction leads to a high current in the rotor circuit and the 
converter, resulting in damage to the DFIG converters [13]. The traditional solution is to use a crowbar system to short-circuit the rotor and protect the rotor-side converter from over current $[13,15]$. However, WTs equipped with active crowbars do not fulfill the latest grid codes, as the machine draws a large amount of reactive power from the power grid, which can exacerbate the voltage sag condition. Moreover, the power unbalance during the fault may excite low-frequency oscillations in the WT shaft system, which results in oscillations of the shaft speed and the output active power [15]. These oscillations are very slowly damped if there is no damping control system, clearly designed for the WT generation system.

\section{Photovoltaic System}

Attempts to reduce greenhouse gas emissions are promising with the recent dramatic increase of installed photovoltaic (PV) capacity, which is predicting a $25 \%$ growth over the next 10 years [17]. Power electronic interface circuit as an inseparable and costly part for utilizing the PV system is required to guarantee the power exchange from the PV source to the grid without violating the grid codes and standards such as CSA-C22.2, UL 1741, IEEE 1547, and IEC 62109-1 [18]. Practically, the grid-connected PV system architectures can be classified as three basic types: centralized inverter, string or multistring inverter, and AC module-integrated converter (MIC) (also called micro-inverter), as shown in Figure 1.3, [19].

Although the centralized inverter can operate at high efficiency with one dc/ac power conversion stage, there are some disadvantages in this structure, such as: 1) each PV module cannot operate at its maximum power point, which results in less energy harvested; 2) additional losses are introduced by string diodes and junction box; and 3) single point of failure and mismatch of each string or PV panel affects the PV array efficiency greatly. The string inverter is a modified version of the centralized inverter. however, it still suffers some of the disadvantages of the centralized inverter, such as high-voltage 


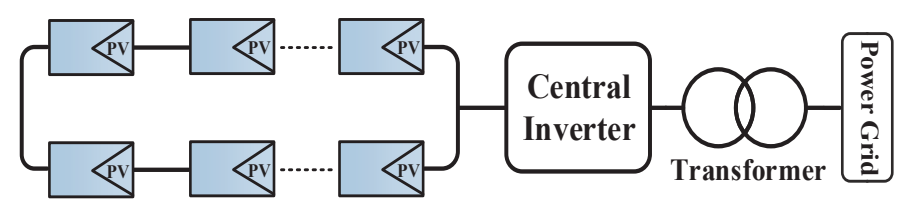

(a)

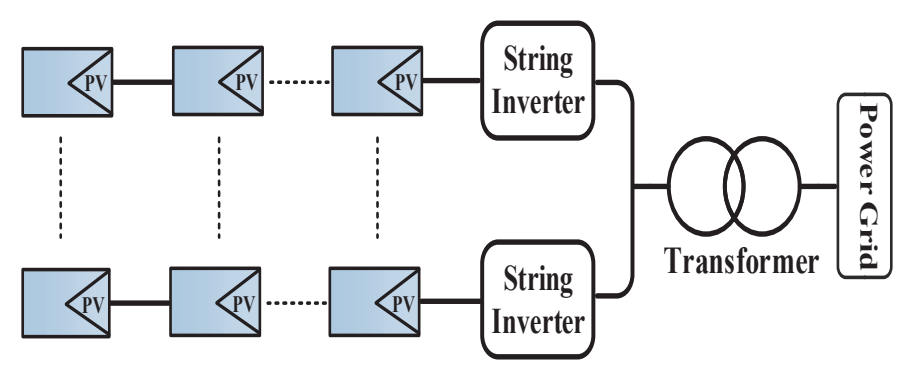

(b)

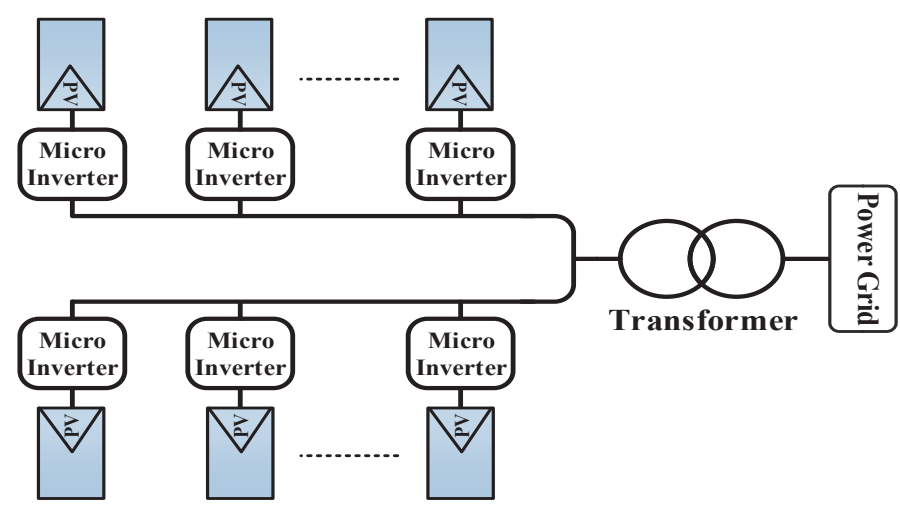

(c)

Figure 1.3: Grid-connected PV system architectures. (a) Centralized inverter. (b) Multistring inverter. (c) AC module-integrated converter (MIC) or also called micro-inverter.

hazard, single-point failure, and difficulty in maintenance. Among these types, the MIC concept is the most recent method for the grid-tied PV system development on the market. There is a growing propensity to use this concept for future solar PV deployment due to its superior advantages, such as the low cost of mass production, high efficiency, easier installation, and improved energy harvest [19]. The PV MIC systems are commercially used in both single-phase and three-phase distributed PV generation, with a power rating range of $150-400 \mathrm{~W}$ and an input dc voltage variation of $25-50 \mathrm{~V}$. 


\section{Protection of Smart Grid Based RES}

The connection of a large number of wind and solar farms to existing distribution networks leads to an increase in the short-circuit capacity of the power system. Due to disturbing the protection coordination, traditional protection methods based on distance and over-current protection will not work successfully. It is thus required to develop smart protection and control actions for ensuring highly reliable and healthy electric power infrastructure by increasing resiliency against component failures or natural disasters, i.e. self-healing ability. Novel superconducting fault current limiter (SFCL) technologies have been launched and introduced into the network as a possible solution to eliminate or largely minimize fault currents [20]. RSFCL is considered as self-healing technology since it eliminates the need for any control action or human intervention due to its automatic excessive current detecting and automatic recovering from non-superconducting to superconducting states. The first-cycle suppression of a fault current by an SFCL results in an increased transient stability of the power system carrying higher power with greater stability. Due to the recent development of lower cost second-generation high temperature superconducting (HTS) wires, the SFCL has become more viable [21].

Among different SFCL devices, the resistive-type SFCL has a simple structure that consists of a lengthy superconductor wire inserted in series with the transmission lines or distribution feeders [22], as shown in Figure 1.4. A parallel resistance to the superconductor is necessary to protect the superconductor from destructive hot spots during the quench. In the United States, development teams and the U.S. Department of Energy (DOE) are developing SFCL prototypes for grid deployment within the next few years [23]. A survey was conducted by Economic Policy Research Institute (EPRI) in 2004 that involved 28 U.S. utility companies. In this report an effort to better understand the level of interest utilities is documented to show progress toward novel FCL devices [22]. Several projects have been conducted by European companies in recen- 


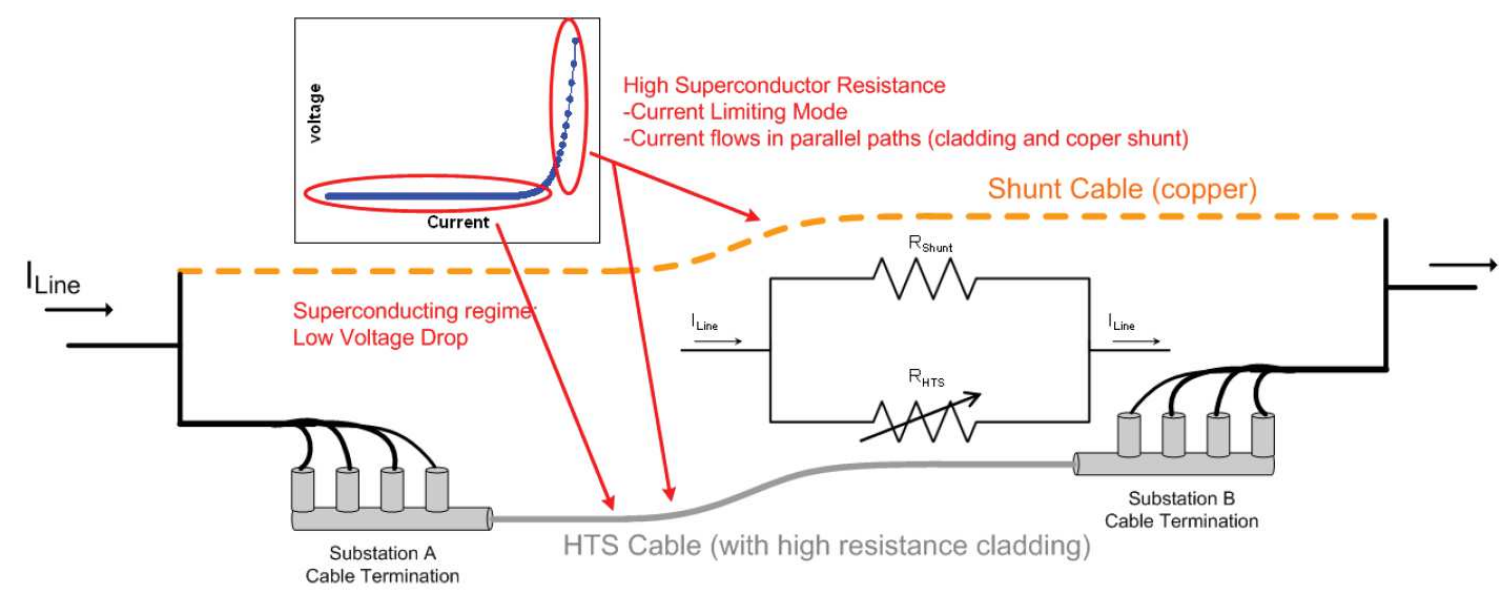

Figure 1.4: Resistive superconducting fault current limiter [22].

t years. A $12 \mathrm{kV} / 16$ MVA Nexans SFCL was installed into the auxiliary power supply system of a large power plant in Boxberg, Germany, in September 2009 [22].

The smart grid is considered as one of the most critical infrastructures of any nation. The interconnection of RESs to the smart grid makes it vulnerable to widespread failures, in which the failure of one node (e.g., substation, transmission line, and communication device) can lead to a cascading failure that propagates to many other interdependent components [24]. One notable example of such propagating failures is the well-known Northeast blackout in 2003 that affected 55 million people and caused an estimated economic loss between 7 and 10 billion [25]. Growth and development of the smart grid has raised serious concerns about the protection of existing power networks. There are an enormous number of such failure events. Although each of them can be analyzed as a causal chain of events after it occurs, it is not feasible to describe all possible causes before happening a severe disturbance. An illustrative example of the smart grid with RES along with its abstracted undirected graph is shown in Figure 1.5, where red stars represent several failure scenarios occur in the smart grid system. Combinations of several types of failures, including failures of protection equipment, transient instability, reactive power problems 


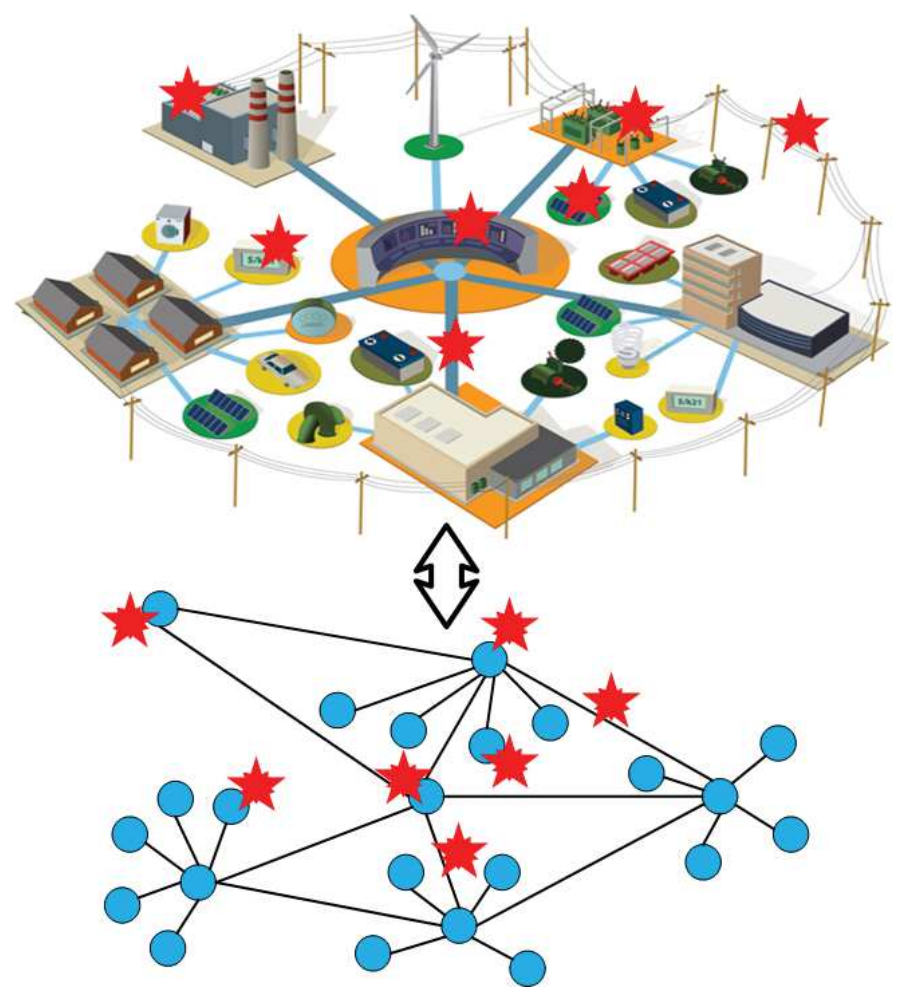

Figure 1.5: An illustrative example of the smart grid with RES and its abstracted undirected graph, where red stars represent several failure scenarios occur in the smart grid system.

and voltage collapse, communication, operational error, and etc, might generally happen in cascading failures. Most works in the power system protection analysis have so far focused on only one of these aspects of failures. Although this way of approach has made possible impressive advances in understanding of each aspect, it does not provide a framework for understanding the overall phenomenon. Since an exhaustive computation of all possible combinations of failures is infeasible, and making a very detailed model of all possible failures and their interactions is beyond the state-of-the-art, compromises are needed in modeling and analyzing the effect of severe multi-failure events on the elements of a smart grid system.

Traditionally, power grid planning techniques encompass N-1 contingencies for protection analysis, in which a single failure event occurs in the grid [26]. However, signifi- 
cant failures might be triggered if concurrent adversarial events are launched to compromise multiple parts of the power grid. Compared with a single failure, multiple failures can have not only serious physical impacts on the smart grid, but they can also potentially deactivate the effect of the operator (decision maker) action mechanisms. In a recent report by the North American Electric Reliability Corporation (NERC) [26], the specified multiple contingencies was identified as one of the three representative high-impact, low-frequency (HILF) threats for power systems.

\subsection{Motivation and Purpose of Research}

The aforementioned technical challenges can be classified into the two major groups. The first group of concerns is related to the interconnection issues between the RES technologies and local networks, addressing active and reactive power support requirements. The second group of concerns is related to the protection issues in the smart grid system with high level of the RESs. The goal of this research is to develop transformative approaches and provide implementable models to enhance technical requirements in terms of protections and power control for integrating of the RES onto the smart grid. In this effort, the advanced and algorithmic methods for specification of the protection scheme, reactive power capability and power control requirements for interconnection of the PV and wind power plants have been proposed. The research was accomplished by utilizing optimization algorithms via multi-objective heuristic optimization techniques and multi-criteria decision making methods to achieve a full penetrative approach in the optimal design for a robust power system.

The research has also proposed the application of superconducting devices, such as superconducting fault current limiters (SFCLs) and superconducting magnetic energy storage (SMES), into the design and optimization of bulk level of renewable resources. The work presented in this dissertation is expected to provide results that can be used 
to explore and develop superconducting technology for integrating very high levels of renewable resources. The SFCL is one unique technology that drew interest after the discovery of high-temperature superconducting (HTS) materials. Moreover, SMES is new equipment that stores electricity from the grid within the magnetic field of a coil containing of superconducting wire with near-zero loss of energy. Recent progress in power electronics technology and the cryogenic systems have made the SMES system more outstanding than any other energy storage systems (ESS), uch as high efficiency (up to 90\%) fast response capability and no deteriorations.

\subsection{Literature Review}

The U.S. government has set up a regional grid code requirement and technical regulations for large renewable power plants. The pros and cons of such regulations have been studied in several publications $[3,27]$. Integration of a huge number of the RES to existing distribution networks leads to an increase in the short-circuit capacity of the power system. Several studies proposed various protection schemes based on differential protection, such as communication-assisted digital relays [28], agent-based paradigm for self-healing protection systems [29] and Distributed Wide Area Differential Protection (DWADP) scheme [30]. In [21], the authors proposed SFCL as a self-healing method to control the fault current levels in utility distribution and transmission networks.

Due to the recent development of cheaper second-generation high high-temperature superconducting (HTS) wires, the SFCL has become more viable [21,30] . A SFCL is an appliance installed in the electric power system for limiting the excessive current by switching into a high-impedance state during a fault event. It has been documented that the SFCL has many advantageous features, such as current detecting and recovering automatically, and faster excessive current damping performances. 
In order to improve LVRT capability of the FSIG-based wind turbine, several methods have been already deployed, such as fast pitching of the turbine blades [31], using commercially available Flexible AC transmission system (FACTS) devices [32], and implementing energy storage systems [33]. Authors in [34] have conducted a comparative study on various transient stability enhancement methods proposed for fixed-speed wind generation systems . It was shown that the pitch control system is the cheapest solution, but it has very slow dynamic response due to the mechanical constraints of the system. Also, the capital cost involved in the installation of the FACTS devices and energy storages is relatively high because of uses of power conditioner systems.

Several studies have been proposed in the literature to limit the dc-link voltage variations and improve the LVRT capability of PMSG-based wind turbines [35-38]. Fast pitch control can help to reduce the input-mechanical power by rotating the blades about their longitudinal axis, also called pitching, and consequently curbs dc-link voltage fluctuations. In [35], a logical fast pitch controller along with fuzzy logic controller (FLC) for back to back converters has been proposed in order to enhance the transient performance of WTs during severe network disturbances. Another solution is to permit the excess wind energy to be temporarily stored in the turbine-generation shaft inertia during the grid faults $[36,37]$. Although, the above techniques are the cheapest solutions for enhancing the LVRT capability of PMSG-based wind turbines, these have a very slow dynamic response due to the mechanical constraints of the system, (the speed of the pitch actuator is slow to contribute alone to LVRT support). The well-known method that is being used for the PMSG-based WT systems is the braking chopper (BC) with the low cost advantage and the simple control performance to consume this surplus power [31,38]. However, in the large wind power plant, including many single wind turbines, the overall cost of using the BCs will be dramatically increased. Moreover, it is difficult to improve the power quality at the output of the wind turbine systems since the BC can just dissipate the power. 
Various control methods are also proposed to ensure proper converter operation for PMSG during fault conditions. The formerly analyzed converter control solutions [37, 39], are based on the classical approach of using the linear proportionalintegral (PI) regulators and pulsewidth/space vector modulation (PWM/SVM). The particular problem is that a linear PI controller is designed for normal network voltage levels, resulting in excessive currents at reduced voltage levels during the fault. The nonlinear control methods are introduced to improve the classical current control method [37,40], but most of these methods are complex and very sensitive to system parameters for practical applications, and need proper tuning of control [41].

Due to intermittent nature of the wind, the power generated cannot follow the load demand effectively, thus few additional energy storages are required to improve the power system stability [42]. The superconducting magnetic energy storage (SMES) system is a new technology that has been recognized as most reliable and effective option to regulate power fluctuations and maintain the stability of the voltage in power system [43]. SMES systems store energy in a magnetic field created by DC current flowing through a superconducting coil that has been cryogenically cooled. Recent progress in power electronics technology and the cryogenic systems have made the SMES system more outstanding to any other energy storage systems (ESS). Generally speaking, the SMES system has a number of superior performances over the DC microgrid, such as high efficiency (up to 90\%), fast response capability and no deteriorations [44]. In recent year, several efforts have been documented on different operational modes and control strategies of VSWTPMSG based DC microgrid along with energy storage system [45, 46]. The SuperPower Inc., in partnership with ABB Inc., Brookhaven National Laboratory (BNL) and the Texas Center for Superconductivity (TcSUH) at the University of Houston, are currently developing an advanced SMES device with demonstrator specifications of $20 \mathrm{~kW}$ ultra-high field (capacity up to $2.5 \mathrm{MJ}$ ), field of up to $25 \mathrm{~T}$ at $4.2 \mathrm{~K}$ and capable of flexible and direct 
deployment in medium voltage distribution networks at $15-36 \mathrm{kV}$. This project is funded in part by an award from the Advanced Research Projects Agency-Energy (ARPA-E) [47].

The majority of the WTs in power systems have been recently equipped with doubly fed induction generators (DFIGs) [13-16]. Since many WTs have been electrically integrated into a weak power grid, the DFIG converters are not able to provide adequate reactive power and voltage support, due to their limited capacity, and thus, there can be a risk of voltage collapse [15]. Therefore, the applications of the static synchronous compensators (STATCOMs) are being increasingly proposed to provide a rapid and controllable reactive power compensation, helping with the uninterrupted operation of WTs under disturbances $[13,15]$. However, a DFIG has a complicated structure including several components, and the inclusion of a STATCOM makes this layout more problematic for the optimal and real-time control of the power system.

The stability analysis and optimal control of the DFIG-based WTs have been studied by many researchers using optimization-based approaches [14,48-53]. In [48], the bacterial foraging algorithm (BFA) is used for the optimal control of a DFIG system. Authors in [49] described a new modified model of the genetic algorithm (GA) for optimal control design of a large offshore wind farm. The particle swarm optimization (PSO) algorithm was proposed for the DFIG-based WT systems to find optimal values of both converter and active damping controllers $[14,50]$. An optimal coordination of the DFIG converters through a fuzzy controller, which is designed using the GA was proposed in [51] to enhance the LVRT capability of WTs. Other advanced coordinated control approaches, such as adaptive dynamic programming (ADP) based methods have shown promising results for such a challenging problem $[52,53]$. Also, the application of a STATCOM to improve the capability of a wind farm (equipped with DFIGs) to ride through grid faults in a multi-machine power system has been reported in [13]. However in [13], there is no coordination between the wind farm and the STATCOM for the reactive power control. 
In [15], authors proposed a heuristic dynamic programming (HDP) based on the coordinated reactive power control of a large wind farm and a STATCOM to reduce the level of the voltage dip during the fault and improve the post-fault power oscillation damping of the system. Although these optimization-based techniques have shown promising results for the proper optimal operation of the system, there are some deficiencies, mainly addressing single-objective optimization problem. In a multi-objective optimization problem (MOP), since several objectives of the system can be simultaneously optimized based on their priority; Pareto-optimal solutions can be obtained instead of a single optimal solution, which represents the best possible trade-off between the objectives $[54,55]$. A decision maker can select any of the Pareto-optimal solutions based on his/her own preference. A breakthrough in obtaining Pareto solutions has been realized by the application of stochastic methodologies, such as GA, PSO, and normalized simulated annealing (NSA) algorithm [21]. Among these algorithms, NSA has the major advantage to avoid becoming trapped at a local minima $[21,54]$.

Practically, the grid-connected PV system architectures can be classified as three basic types: centralized inverter, string or multistring inverter, and AC module-integrated converter (MIC), as shown in Figure 1.3. Among these types, the MIC concept is the most recent method for the grid-tied PV system development on the market. There is a growing propensity to use this concept for future solar PV deployment due to its superior advantages, such as the low cost of mass production, high efficiency, easier installation, and improved energy harvest [56]. The PV MIC systems are commercially used in both single-phase and three-phase distributed PV generation, with a power rating range of $150-400 \mathrm{~W}$ and an input dc voltage variation of $25-50 \mathrm{~V}$. Since the low PV voltage needs to be boosted to match the utility grid voltage, several MIC topologies based on the number of conversion stages and the design requirements have been studied and presented in the literature [19,57-59]. Figure 1.6 summarizes the most commonly used configura- 


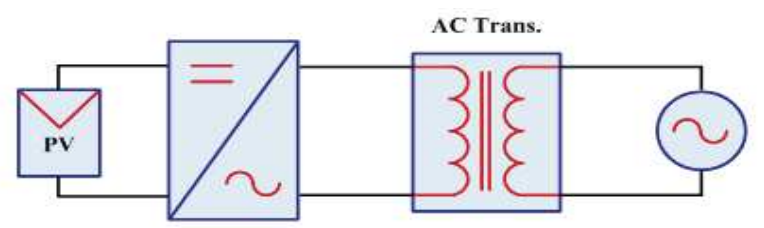

(a)

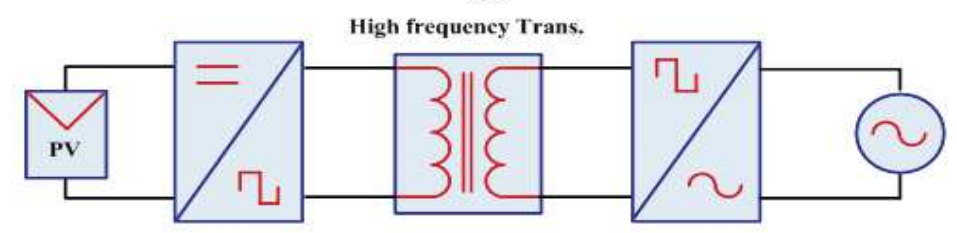

(b)

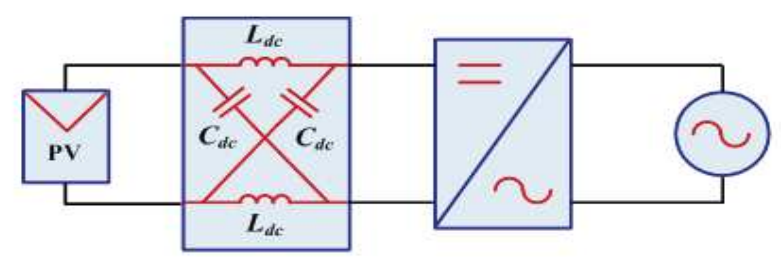

(c)

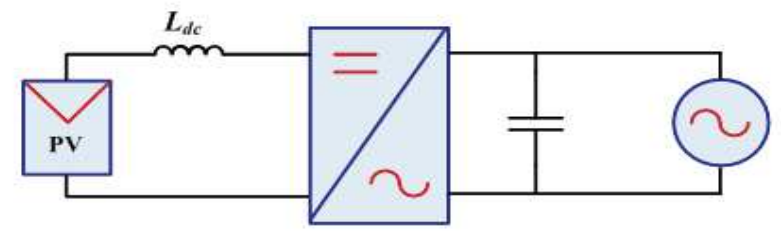

(d)

Figure 1.6: Four options for connecting PV MIC systems. (a) MIC with line-frequency transformer. (b) MIC with high-frequency transformer. (c) Single-stage z-source MIC. (d) Single-stage current source MIC.

tions in the presence of PV MIC industry. In Figure 1.6(a), a line-frequency transformer is employed to boost the voltage after the voltage source inverter (VSI). However, a lowpower transformer is bulky with load acoustic noise and may not be very efficient. In Figure 1.6(b), a high-frequency transformer is introduced to obtain the voltage amplifications. Since high switching frequency is essential to achieve the compact inverter size, the switching loss of the semiconductor devices and the transformer loss are the dominated limitations for the efficiency improvement [57]. Figure 1.6(c) shows the z-source inverter as another solution which has the capability of boosting and inverting the dc voltage in a single stage with few solid-state switches. However, this topology has relatively high in- 
put current ripples, resulting in high stresses on the dc-link inductors and capacitors [58]. A single-stage topology based on the current source inverter (CSI) is depicted in Figure 1.6(d). The single-stage CSI topology is an alternative method to achieve the dc voltage boost capability without additional stages which will reduce the circuitry complexity and the overall system losses, as discussed in [59]. Although the CSI has been well documented for high-power drive and rectifier applications, but it is rarely considered in low power ranges (up to $500 \mathrm{~W}$ ) especially in PV MIC industry [60-62]. Recently, development of the three-phase MIC concept to large-size PV installations is introduced where each MIC is integrated directly into each PV panel [63]. This architecture will reduce the cost per watt, improve system reliability, and eliminate the single-point failure. Assuming further expansion of the three-phase MIC system, it would be required for MIC to be equipped with real-time active and reactive power control capability to fulfill the upcoming grid requirements.

The previously analyzed control schemes are focused on the current regulation of the two-stage VSI-based MIC systems, which are mostly based on the concept of the $d q$ rotating synchronous reference frame with the conventional linear proportional-integral (PI) regulators and PWM technique. The PI-based current regulation approach was originally proposed in [64] and was extensively studied and adopted for the current control of both single- and three-phase systems in various applications [65-67]. In the design of the PI controller, two distinct $d$ - and $q$-axis currents must be extracted in order to be independently regulated. However, due to the poor disturbance rejection capabilities, the $d$ and $q$ axes are not fully decoupled, and the step changes in one axis generate transients in the other, which might lead to power quality and performance deterioration. To completely decouple $d$ - and $q$-axis currents, a multivariable PI controller with a faster dynamic response and superior axis decoupling capability was proposed and experimentally evaluated for a three-phase VSI [68]. 
After a large blackout occurs, considerable efforts, based on optimization methods and simulation tools, are made to analyze the detail of that particular cascade and improve the power system protection in order to minimize the chance of a similar cascade happening. However, severe multi-failure events are a challenge to analyze and simulate in a predictive way due to the huge number of possible interactions and the diversity and complexity of these interactions. At the same time, it is recognized that the current methods for directly understanding and mitigating severe multiple failures are not well developed. In the literature, several approaches for analyzing critical multiple contingencies have been recently proposed based on simulation methods and optimization theories to determine vulnerabilities of the power system to worst-case scenarios [69-74]. A dynamic decision tree along with fast simulation was developed in [69] to practically compute high-risk contingencies. In [70], a fast simulation of hidden failures was presented to identify critical relays, which contribute to many possible cascades. A hidden failure is undetectable during normal operation but will be exposed as a direct consequence of other system disturbances, which might cause a relay system to incorrectly and inappropriately disconnect circuit elements. Optimizations methods in $[71,72]$ were used to minimize the blackout due to contingencies caused by a failure with finite resources. Authors in [73] presented a graph theoretic method in order to find critical lines and cutlets by considering load-generation mismatch. The work in [74] proposed a partitioned multi-objective risk scheme to access tradeoffs between N-1 security and survivability under catastrophic failure events, considering various levels of resiliency from low to high damage severity, while minimizing the cost. Although the above quantitative studies provide some good insights, generally, fast simulation and random analysis of the power systems cannot precisely explore the critical multiple contingencies as worst-case scenarios, due to the huge number of possible interactions and the diversity and complexity of these interactions. More recently, the application of game-theoretic approaches for the power grid protec- 
tion, such as in [75-78], have attracted attention due to the ability of such frameworks to model and analyze the decision making processes involved in power system protection. In [75], the potential of applying game theory for the power grid was proposed in three merging areas including: microgrid systems, demand-side management, and communications. A Markov game model was introduced in [76] to analyze the resource allocation for transmission lines protection of the smart grid. In [77], the risk assessment of cyber failure against the smart grid was proposed based on the noncooperative game theory. A static game model was proposed in [78] to achieve optimal responses for power grid operators in a game against nature that performs severe component failures. However, [78] relies on a static game formulation, in which the dynamics of the power grid are ignored and the interactions between the failure events and the operator responses are assumed to be one-shot events. Thus far, to our best knowledge, there have been no reports, fully considering the power system survivability scheme under severe multi-failure events, using stochastic game theory.

\subsection{Research Objectives and Original Contributions}

The research reported in this dissertation conducted analysis and modeling of the advanced power control and protection requirements for reliable RES integration onto a smart grid system. The thesis has completed the following five major activities:

\section{1) Investigation of the low voltage ride-through (LVRT) improvement methods for fixed-speed induction generator (FSIG)-based wind turbines (WTs)}

Since a non-negligible $20 \%$ of the existing wind energy is still employing FSIGs due to their simple structure and lower maintenance cost, the fault-ride through characteristics of FSIG-based wind turbines still need to be analyzed. Through review of the existing LVRT improvement methods for FSIG-based WT, it was determined that there is no valu- 
able review studies, which fully consider LVRT enhancement strategies of this type of wind generation. This dissertation fills this void by providing a comprehensive analysis of existing LVRT methods in terms of dynamic performance, controller complexity, and economic feasibility. A new feature of this effort is to categorize LVRT capability enhancement approaches into three main groups depending on the connection configuration: series, shunt, and series-shunt (hybrid) connections, and then discuss their advantages and limitations in detail.

\section{2) Application of superconducting devices for optimal performance of renewable energy sources (RES)}

There have been few reports on the application of superconducting devices into the design and the optimization of bulk level of renewable resources. This effort has presented the application of superconducting technologies into the body of the thesis. This was achieved by developing accurate mathematical electro-thermal derivations for the superconducting fault current limiter (SFCL) and superconducting magnetic energy storage (SMES), which are validated via simulation results. It included three sub-objectives:

\section{2-1) LVRT enhancement of permanent magnet synchronous generator (PMSG)-}

\section{based WTs using SFCL}

This research has demonstrated that the resistive SFCL is a promising solution for the WT controller performance with respect to dc-link fluctuations and extreme loads happening to electrical and mechanical parts, respectively, during the severe disturbances. The further effort has been centralized on the most-widely decision-making technique based on the analytic hierarchy process (AHP), for the optimal performance of the combinatorial RSFCL and PMSG-based WT. 


\section{2-2) Power management of DC grid involving PMSG-based WTs and SMES}

A power balance management between loads, a micro-SMES system and a nondeterministic wind generation based on PMSG has been maintained via presenting the supervisory control architecture. For this purpose, an optimal design scheme based on simulated annealing (SA) algorithm has been modeled for micro-SMES solenoid coil to ensure the desired energy storage capacity with least volume.

\section{3) Implementation of the multi-objective optimization model for designing a new on-} line coordinated reactive power control between DFIG-based WT and STATCOM.

The key motivation of this study is to design a coordinated reactive power controller for combinatory DFIG-based WT and a static synchronous compensator (STATCOM) that is formulated as a multi-objective optimization problem (MOP). The proposed MOP model is solved by using the stochastic normalized simulated annealing (NSA) algorithm in order to find the Pareto-optimal solutions, according to the assigned priorities (weights) for each objective. A fuzzy logic controller (FCL), which is properly tuned by the NSA algorithm, has been introduced for the online application.

\section{4) Development of the simplified power control approach with reliable axis decou-} pling capability for three-phase current source inverter (CSI)-based MIC system

This objective includes two original contributions. First, a current source inverter (CSI) with dc voltage boost capability, namely the single-stage power conversion system, has been proposed for a three-phase PV MIC system. A switching pattern based on the phasor pulse-width-modulated (PPWM) technique has been employed to produce the switching signals through introducing the modulation index and the modulation angle. Furthermore, a current control strategy based on multivariable-proportional-integral (PI) regulators with structural simplicity and fast dynamic response has been designed to control the proposed 
CSI-based MIC system. The performance of the proposed control system has been experimentally verified through a 300-VA laboratory prototype.

\section{5) Development of the new game-theoretic framework to provide reliable strategies} for protecting smart grid components under concurrent multiple failures.

The main contribution of this objective is to introduce and develop a new game-theoretic framework to analyze how a smart grid can dynamically survive concurrent severe multifailure events. This has been accomplished by proposing a new learning algorithm to obtain the operator's Nash equilibrium strategies that provide insightful guidelines on how to deploy limited budget for protecting critical components of the power grid.

\subsection{Dissertation Organization}

The rest of this dissertation is organized as follows: Chapter 2 presents a comprehensive review of various techniques, which are employed to improve the low voltage ride through (LVRT) capability of the fixed-speed induction generators (FSIGs)-based wind turbines (WTs). The reviewed methodologies are classified into the three main groups, namely, (i) series-connected solution; (ii) shunt-connected solutions; (iii) hybrid-connected solutions. A comparative study in terms of dynamic performance, controller complexity, and cost evaluation of these LVRT methods is then carried out. Finally, simulation results of several LVRT methods are illustrated to compare the dynamic performance of the wind turbine equipped with auxiliary devices.

Chapter 3 introduces the application of the superconducting fault current limiters (SFCLs) and superconducting magnetic energy storage (SMES) into the smart grid system based on RES. This chapter includes three sections. Section 3.2 explores a FSIG-based WTs in an effective combination with a UPQC and a resistive SFCL. Section 3.3 presents a positive approach using a SFCL for the LVRT enhancement of the PMSG-based large wind power plant (WPP). Finally, Section 3.4 presents a combinatorial standalone PMS- 
G based variable speed wind turbine (VSWT) and small-size SMES system into the DC microgrid system.

Chapter 4 proposes a computational intelligence technique for optimal coordinated reactive power control between a WT equipped with DFIG and a STATCOM, during faults. The proposed coordinated reactive power control is formulated as a multiobjective optimization problem (MOP) model to minimize the voltage deviations at the WT terminal during and after the faults and active power oscillations after clearing the faults. A normalized simulated annealing (NSA) algorithm is employed to find pareto-optimal solutions of the MOP model. Next, a fuzzy logic controller (FLC), which is is properly tuned by the NSA algorithm, is proposed for the online application. Finally, the performance of the proposed control approach is successfully validated with simulation results.

Chapter 5 discusses the application of a three-phase module-integrated converter(MIC) incorporated into a grid-tied photovoltaic (PV) system. A current source inverter (CSI) with dc voltage boost capability, is proposed for a three-phase PV MIC system. A switching pattern based on the phasor pulse-width-modulated (PPWM) technique is employed to produce the switching signals. Then, the state-space averaging method is utilized to derive the large- and small-signal models in order to study the dynamic behaviors of the CSI-based MIC system. Finally, the performance of the proposed multivariable PI control method has been simulated in MATLAB/Simulink software and then experimentally verified through a 300-VA laboratory prototype.

Chapter 6 develops a new game-theoretic framework to explore reliable strategies for power grid operators in order to protect the smart grid under multi-failure events, by restoring some links before the failures spread to other parts of the system. First, The stochastic game framework is described and analyzed. The two algorithms to obtain Nash equilibrium strategies for power system operators are presented in next, while some illustrative examples and case studies are given in this chapter. 
Chapter 7 summarizes the dissertation outcomes, concludes the significance of this research, discuss the results, and finally makes recommendations for the future works. 


\section{CHAPTER 2}

\section{A COMPREHENSIVE INVESTIGATION OF LVRT IMPROVEMENT METHODS FOR FIXED-SPEED WIND POWER GENERATORS}

\subsection{Overview}

This chapter presents a comprehensive review of various techniques, which are employed to enhance the low voltage ride through (LVRT) capability of the fixed-speed induction generators (FSIGs)-based wind turbines (WTs). The main contributions of this chapter are organized into the following sections: after describing the operation of the FSIGbased WT under normal and faulty conditions in Section 2.2, the comprehensive review of the recently LVRT capability improvement approaches is discussed in Section 2.3, 2.4, 2.5. The reviewed methodologies are classified into the three main groups, namely, (i) series-connected solutions (e.g., thyristor-controlled series compensation (TCSC), dynamic voltage restore (DVR), series dynamic braking resistor (SDBR), magnetic energy recovery switch (MERS), and fault current limiter (FCL)); (ii) shunt-connected solutions (e.g., static var compensator (SVC), static synchronous compensator (STATCOM), and superconducting dynamic synchronous condenser (SDSC); (iii) hybrid-connected solutions (e.g., unified power quality conditioner (UPQC), and unified compensation system (UCS). A comparative study in terms of dynamic performance, controller complexity, and cost evaluation of these LVRT methods is carried out in Section 2.6. In Section 2.7, simulation results of several LVRT methods are illustrated to compare the dynamic performance of the wind turbine equipped with auxiliary devices.

\subsection{FSIG-based wind turbine}

A non-negligible number of WTs, which are equipped with induction generators, are still in the operation at an almost fixed speed due to wide usage of early wind turbine systems. These were the first Danish wind turbines, which completely dominated the market up to 


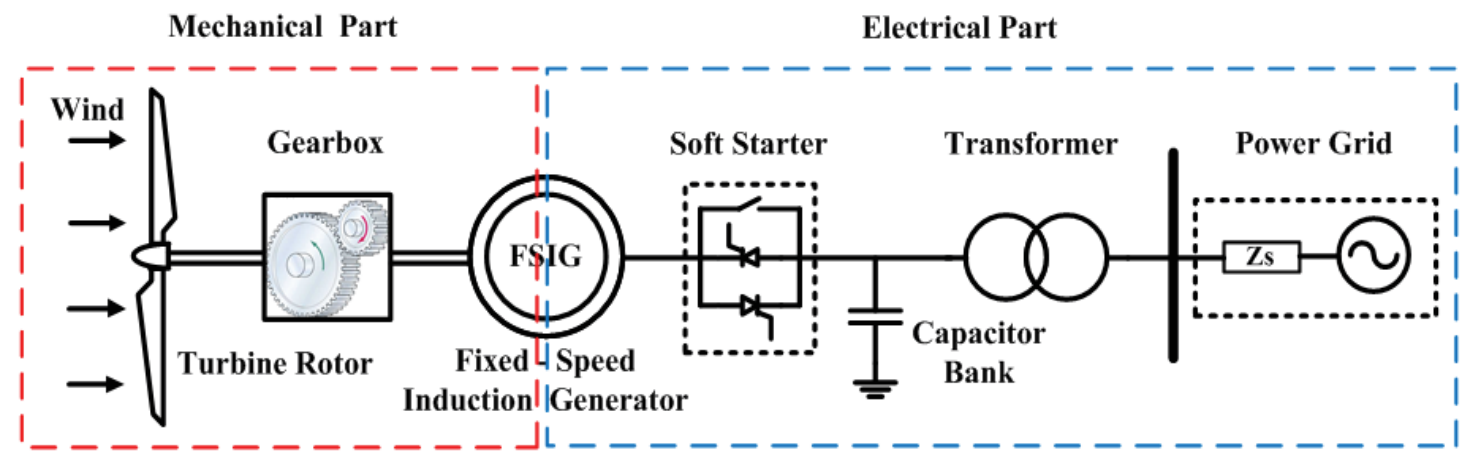

Figure 2.1: The main components of the wind turbine based on FSIG.

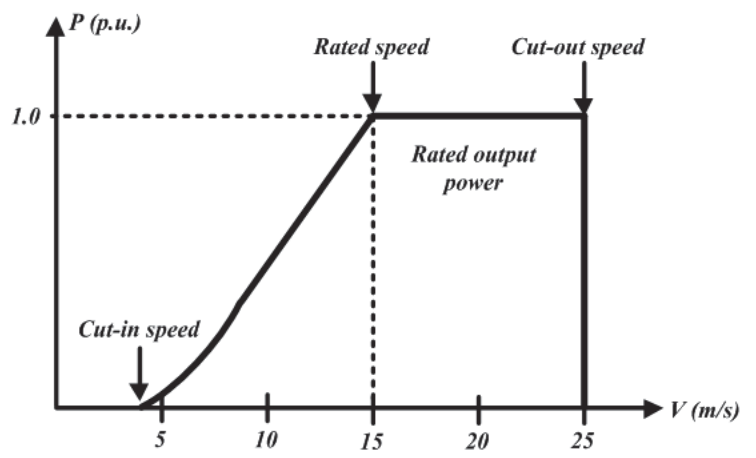

Figure 2.2: The output power of a wind turbine as a function of the wind speed.

the mid-1990s (the reason why this technology is named the Danish concept [79]. The basic configuration of the WT based on FSIG is shown in Figure. 2.1, including a turbine rotor, gearbox, squirrel-cage induction generator, soft-starter, mechanical-switched capacitors (MSCs), and a transformer for grid connection.

\subsubsection{Steady-state operation}

WTs extract the power from the wind via aerodynamically designed blades and convert it to mechanical power. Under high wind speed conditions, the power delivered by a WT may exceed its rated value. Thus, an effective approach must be applied to reduce a portion of the wind power so as to avoid turbine damage. The aerodynamics of fixed- 


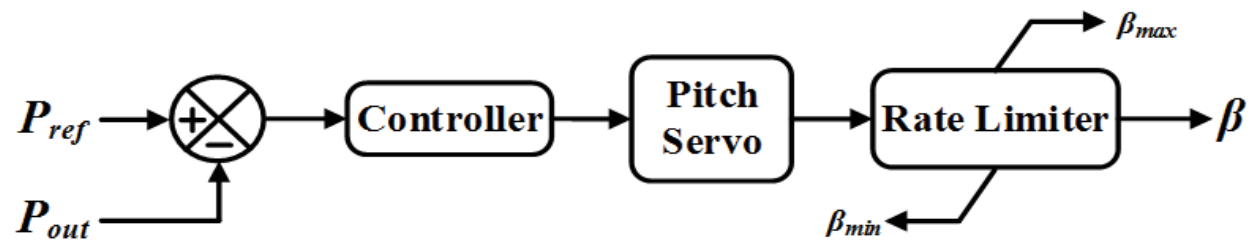

Figure 2.3: Conventional pitch angle control used in FSIG-based wind turbine.

speed wind turbines can be controlled by passive stall, active-stall, and pitch control approaches, which have been developed for low, medium, and large WTs, respectively [80]. An example of the relationship between the wind speed and the power generated by the wind turbine is shown in Figure 2.2 [81]. The blades start to move around $4 \mathrm{~m} / \mathrm{s}$, while an optimal aerodynamic efficiency is achieved at the wind speed rated about $15 \mathrm{~m} / \mathrm{s}$. The extra power obtained from wind speed between 15 and $25 \mathrm{~m} / \mathrm{s}$ may be smoothly curtailed by spinning the blades using either active stall or pitch control to avoid overloading the wind turbine system. Over the cutout wind speed, the turbine has to be disconnected in order to avoid damage. It has been reported that the pitch control has become dominant in the wind power market (used four times more than the stall control) [82].

Many methods of WT pitch control have been documented, such as the classical proportional-integral-derivative (PID) control [83], gain-scheduling control [84], or robust control [85], among other nonlinear controllers [86]. Figure 2.3 depicts the conventional pitch angle regulator in which the input and output of the model are the generated power $P_{\text {out }}$ and blade angle $\beta$, respectively.

The gearbox plays an essential role in the fixed speed WTs to adapt low-speed, hightorque rotation of the turbine rotor into the faster rotation of the electrical generator [82]. The critical issue in implementing the gearbox technology is the wind gusts and turbulence, which may lead to misalignment of the drive train and a gradual failure of the gear components, consequently increasing the capital and operating cost of the WTs. Induction generators with a squirrel-cage rotor can be used in the fixed-speed WTs at low 
cost and with low maintenance due to rugged brushless construction [79,87]. Nevertheless, it has some important drawbacks, as it requires a stiff power grid to enable a stable operation, and may also require a more expensive mechanical construction in order to absorb the high mechanical stress, since wind gusts can cause torque pulsations on the drive train [81]. Moreover, the induction generators tend to draw a large amount of the reactive power from the grid in their steady-state operation for self-excitation because stator windings are directly connected to the grid with no power electronics [88]. Thus, the low-cost mechanically switched capacitor (MSC), which generally includes a bank of shunt capacitors, are connected to the terminal of the wind generator to achieve a unit power factor and to provide a voltage regulation in normal operation conditions [89].

The high starting inrush currents generated by the connection of induction generators to the power system may cause disturbances to both the grid voltage and high torque spikes in the drive train of WTs. Thus, current limiters or soft-starters based on thyristor technology are used to typically limit the rms value of the inrush currents to a level below two times of the generator rated current [81]. Further, the soft-starters effectively dampen the torque peaks associated with the peak currents, and hence reduce the loads on the gearbox.

\subsubsection{Transient-state operation}

When the fault starts, the voltage at the terminals of the WT drops down, thus leading to the significant reduction in the electromagnetic torque and the electric-power output of the induction generator. However, the mechanical-input torque remains constant during the fault, causing the rotor speed to increase beyond its safety limits in order to mechanically store the energy excess. Therefore, it is important to keep the balance between the mechanical-input power and the electrical-output power for enhancing the fault ridethrough capability of the FSIG-based WT. 
After the fault clearance, since the rotor speed increases during the fault period, a large amount of reactive power is absorbed by the induction generator from the grid. As a consequence, not only the induction generator is unable to fulfill the reactive power injection requirements along the fault, but also it exacerbates the voltage sag condition by absorbing the reactive power, so that it creates difficult situations to restore the terminal voltage within the acceptable level. As illustrated in Figure. 2.3, if $P_{\text {out }}$ exceeds its rated value, the pitch angle $\beta$ increases to limit the generated wind power to its rated value. Under fault conditions, the generated power abruptly falls and it is unable to perfectly contribute in the LVRT improvement.

To overcome these drawbacks, the modified pitch angle controllers are proposed in the literature based on the wind generator speed, so that these can immediately increase the pitch angle to reduce the mechanical-input torque, as shown in Figure 2.4. [31, 90] . Although the pitch control system is the cheapest solution for enhancing the LVRT capability of the FSIG-based wind turbine, it has a very slow dynamic response due to the mechanical constraints of the system [91]. Also, MSCs represent relatively weak performance during fault conditions, owing to the decrease of their reactive power injection capability following voltage drops. Furthermore, the excessive switching of the capacitor bank provokes failures, applying the inherent voltage steps stress on the wind turbines, and increases the required maintenance of the system [81].

The above discussions have covered the symmetrical grid faults, but in general the majority of the grid faults result in an asymmetrical voltage dip with both positive and negative sequence components. When the grid voltage is unbalanced, i.e. it contains a fundamental negative sequence component, the stator current of the induction generator becomes unbalanced as well. As stated in [92], a slight amount of negative-sequence voltage causes higher amounts of negative-sequence currents, and consequently creates the additional torque oscillations of the double grid frequency. It also results in heating 


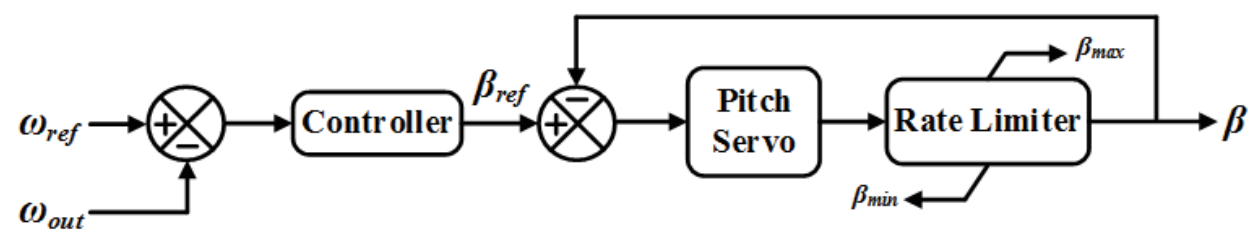

Figure 2.4: Modified pitch angle control used in FSIG-based wind turbine.

of the stator windings, and thus reduces the life span of the gearbox, blade assembly, and other components of a typical WT $[93,94]$. The magnitude of the negative and positive sequences of the torque can be calculated, as follows

$$
\begin{aligned}
& T^{+} \simeq 3 \times \frac{p}{2 \omega_{s}} \times V_{s}^{+} \times I_{s}^{+} \\
& T^{-} \simeq 3 \times \frac{p}{2 \omega_{s}} \times V_{s}^{+} \times I_{s}^{-}
\end{aligned}
$$

where $T^{+}$and $T^{-}$are the positive and negative torque sequences, $p$ is the number of poles, $\omega_{s}$ is the sliding angular frequency, $V_{s}^{+}$is positive-sequence voltage, $I_{s}^{+}$and $I_{s}^{-}$are positive and negative current sequences, respectively. It is clear from (2.1) and (2.2) that the average torque is reduced due to the decrease of the positive-sequence voltage, which leads to the acceleration of the induction generator, mechanical vibrations, and acoustic noise. The response of WTs based on the FSIG during asymmetrical faults have been investigated in the literature, while several control methods, injecting negative sequence current, have been proposed to balance the grid voltage by reducing the negative sequence voltage [94-97]. As discussed previously, the LVRT performance of FSIG-based wind turbines is a problematic issue because the stator windings are directly coupled to the grid, and the induction generator consumes reactive power during and after a fault. Therefore, it fails to fulfill some of the important grid integration requirements, such as the reactive power compensation or the terminal voltage control. Thus, the induction generators need the external supporting devices to avoid their tripping during the voltage reduction. 


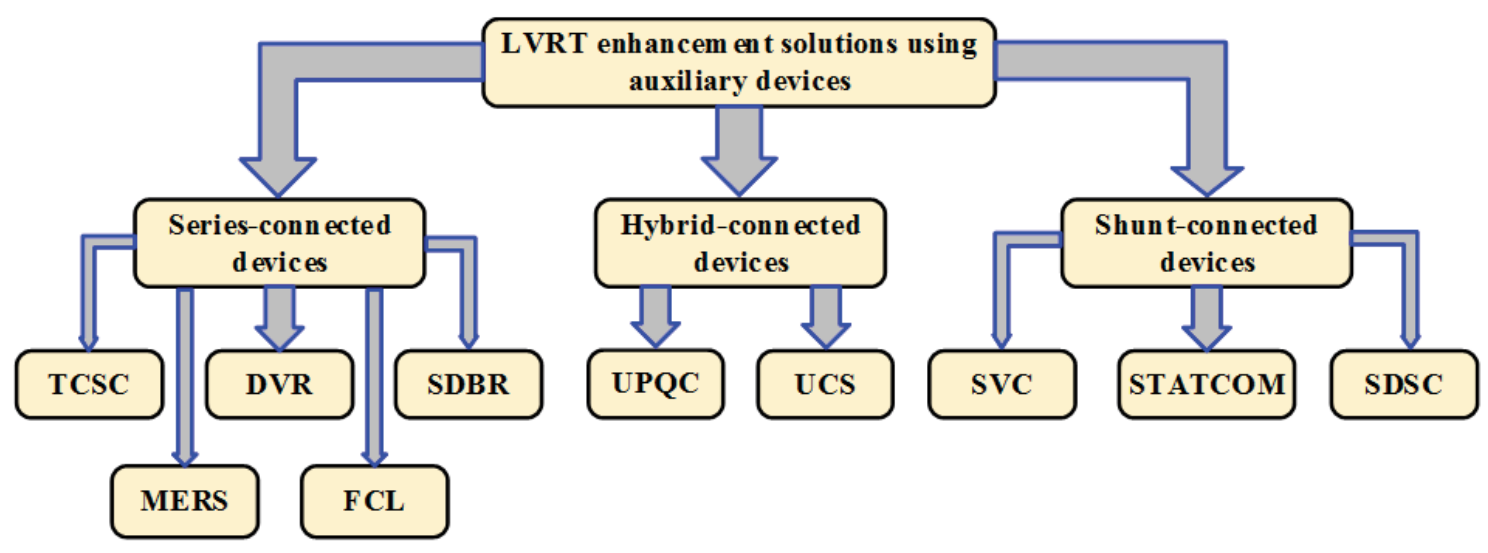

Figure 2.5: Classified LVRT capability enhancement methods.

There are many auxiliary devices reported in the literature to provide an adequate dynamic voltage support and enhance the LVRT capability of WTs. The major categories of LVRT methods for the FSIG-based wind turbine are depicted in Figure 2.5. Depending on the connection configuration, these methods can be classified into the series-connected solutions, shunt-connected solutions, and hybrid-connected solutions.

\subsection{Review of series-connected solutions}

Series-connected auxiliary technologies have been successfully implemented to alleviate grid congestion, defer construction of new transmission lines, and improve system capacity. These types of technologies, as a relatively simple solution, with a smaller current injection compared to shunt-connected technologies, are effectively used to regulate voltage or limit fault current, resulting in a significant increase in the transient and voltage stability in transmission systems. A brief explanation of series-connected solutions is presented in the following subsections.

\subsubsection{Thyristor-controlled series compensation (TCSC)}

The essential principle of the TCSC is to control the power flow of the grid lines, to increase the dynamic stability of power transmission, and to effectively limit the power os- 


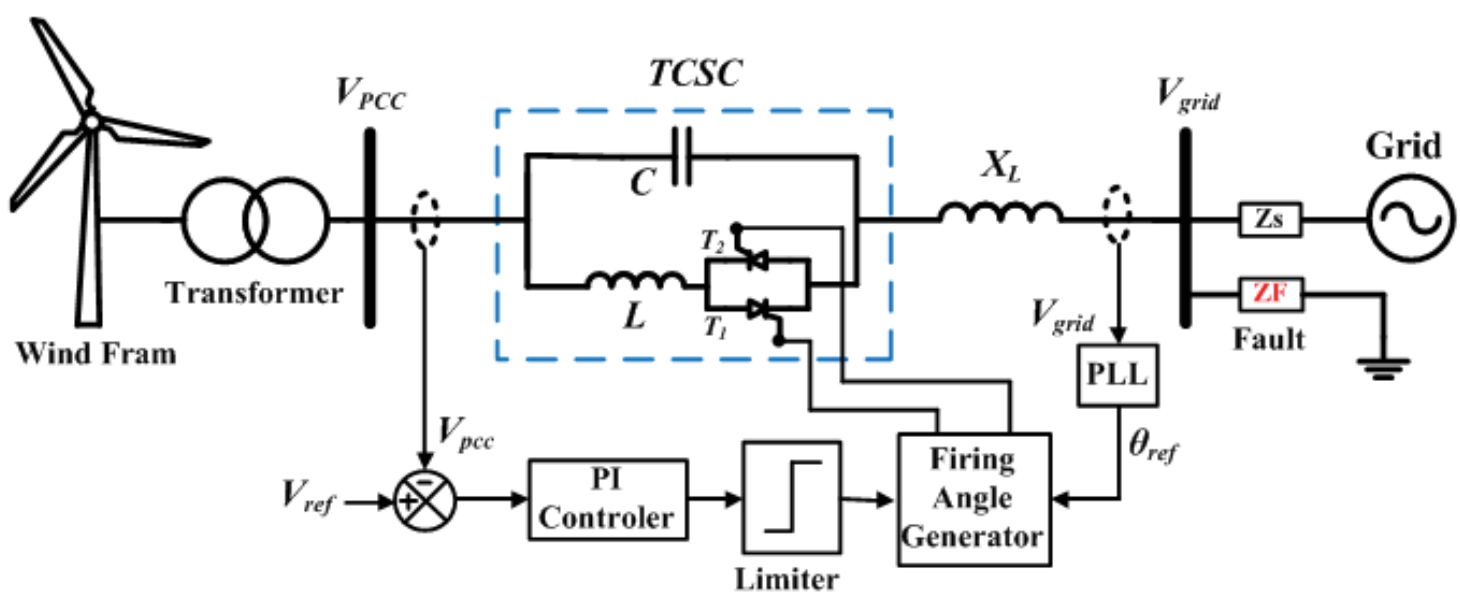

Figure 2.6: TCSC module installed outside the wind farm with the basic control scheme.

cillations [98-100]. These features have been effectively proven by existing installations of TCSCs described in the literature, such as the Western Area Power Administration's Kayenta site [100], or the Bonneville Power Administration's Slatt substation [101]. Recently, the abilities of this technology have particularly been realized where inconstancy in the transmission lines for delivering the huge WT generated power into the grid, which led to the voltage collapse and cut off the fixed speed WT [102,103]. Moreover, the ability of TCSC to limit the fault current and control the voltage unbalance of wind farm systems is discussed in [104]. Figure 2.6 illustrates a typical TCSC module installed outside the wind farm along with the basic control scheme. A TCSC consists of three components: capacitor banks $C$, bypass inductor $L$, and forward-biased thyristors $T_{1}$ and $T_{2}$. The control scheme has been well-documented in the literature $[98,99]$. The function of the control block is to generate the appropriate gate drive signals for the thyristors when the fault is initiated. Basically, thyristors are fired with respect to zero crossing of the line current in order to inject the additional current into the capacitor through the bypass inductor, and increase the capacitive reactance value, typically up to a factor of three times the original reactance. Thus, a variable capacitive reactance can be obtained to compensate the reactive power absorbed by the induction generator and improve the 


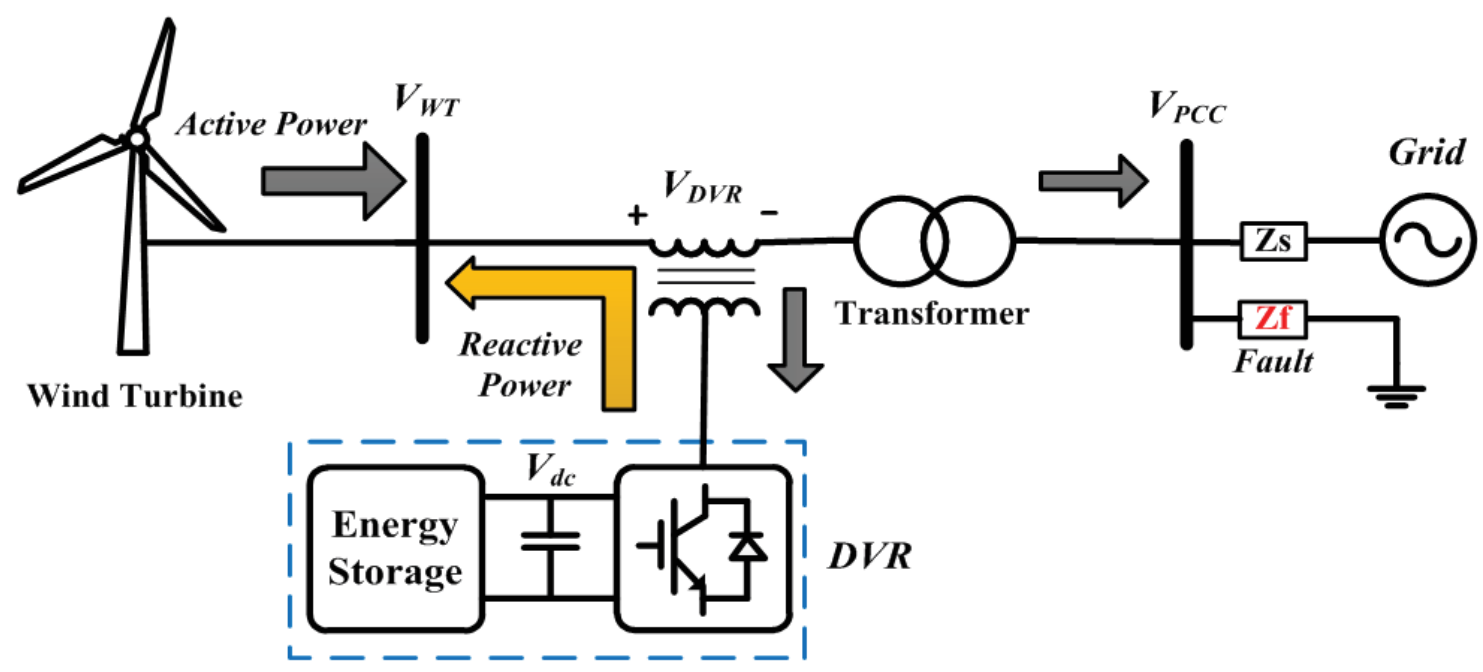

Figure 2.7: Principle operation of the dynamic voltage restorer and power flow during the voltage dip compensation.

fault right through of the WTs. This technology may be useful for wind farms, which are located far away from the PCC, such as offshore wind farms [81].

\subsubsection{Dynamic voltage restorer (DVR)}

A promising approach to effectively overcome the grid-fault-derived problems with WT generators is to control the connection-point voltage by compensating voltage fluctuations during the fault. This can be accomplished by using a series-connected power electronic compensator, called the dynamic voltage restorer (DVR), which injects an appropriate voltage into the grid bus in order to keep the generator voltage constant at PCC with the same phase as the network, as shown in Figure 2.7. Depending on the time frame assumed by the regional grid code (e.g. in the Danish electrical system, $80 \%$ three-phase voltage-dips should be ride-through for up to 30 grid-cycles), a DVR might has a sufficient energy storage capacity to generate the missing voltage at the WT terminal during the dips. There are several efforts that demonstrate the utilization of a DVR for voltage dip mitigation and the voltage recovery, in which DVR restores the WT's terminal voltage to the operating point within the shaded area of the LVRT curve $[105,106]$. However, using 


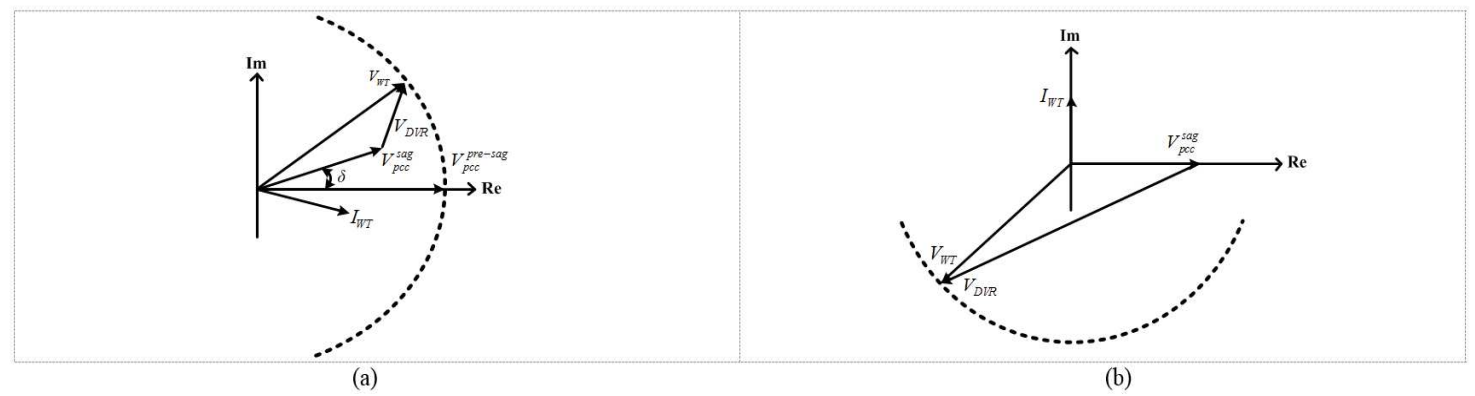

Figure 2.8: Single-phase vector diagram. (a) Voltage dip compensation with DVR. (b) Voltage dip compensation once the phasor has been rotated.

a DVR for voltage sag mitigation in fixed-speed wind generators has certain technical challenges [107]. According to the voltage vector diagram shown in Figure 2.8(a), the voltage dip is causing not only a reduction in the voltage magnitude, but also a change in the phase, which is described as a phase angle jump $\delta$ (phase angle difference between the voltage phase during the sag and the one before the sag)

$$
\delta=\arctan \left(\frac{X_{F}}{R_{F}}\right)-\arctan \left(\frac{X_{s}+X_{F}}{R_{s}+R_{F}}\right)
$$

where, $Z_{s}=R_{s}+j X_{s}$ and $Z_{F}=R_{F}+j X_{F}$ are gird impedance and fault impedance, respectively.

The phase-angle jump reveals itself as a shift in zero crossing of the instantaneous voltage, causing a large transient at the beginning and the end of the sag because the internal generator flux is out of phase with the voltage [106]. Moreover, the DVR is require to absorb the part of the extra active power, which is generated by the wind generator during the fault in order to keep dc-link voltage $\left(V_{d c}\right)$ at the desired level. Thus, it must have energy dissipation capabilities which is the main drawback of the DVR.

To address the aforementioned problems, some successful control schemes are discussed in the literature [106-109]. In the work described in [106], the energy dissipation was accomplished by using a resistor, which is connected to the dc link through a pow- 
er electronic switch, when the dc-link voltage exceeds its safety limits. The decoupled control of $d$ - and $q$-axis voltages have been reported in $[107,108]$ for the DVR inverter to improve the LVRT capability of the FSIG based WTs. In [109], the authors propose an adaptive control system based on the proportional resonant (PR) controller to provide the voltage and current decoupling in order to improve the DVR output voltage tracking capability. In [106], Dionisio et al. carried out a control scheme based on a two-step strategy. First, the DVR compensated the voltage sag to maintain the magnitude and phase of the wind generator voltage at $1 \mathrm{p} . \mathrm{u}$ and second, the control system gradually rotated the series voltage supplied by the DVR, $V_{D V R}$, in order to inject the reactive power into the grid, while the magnitude of the wind generator voltage was kept at 1 pu (see Figure $2.8(b))$.

\subsubsection{Series dynamic braking resistor (SDBR)}

The concept of series-connected dynamic braking resistors (series-DBRs) in the wind power application was introduced early by the authors in 2004-2005 [110]. DBRs have been developed to contribute directly to the balance of active power between the mechanical and electrical side of the WT system during a fault, potentially reduce or eliminate the need for the pitch angle control or the reactive power compensation (RPC) devices [110]. This is performed by dynamically installing a resistor in series between the WT and the grid, in order to boost the voltage at the terminals of the generator, and thereby alleviate the instability concerns on electrical torque and power during the fault period [111].

The typical schematic layout of SDBR may incorporate one or two stages of resistor/switch units, as shown by Figure 2.9(a) and (b), including the static bypass switch, allowing sub-cycle response and smooths variable control. Under normal conditions, the dynamic braking resistor must be cut off by closing the bypass switch. At the beginning of the fault, the current starts the passing through the resistor, $R_{s h}$, and continues in oper- 


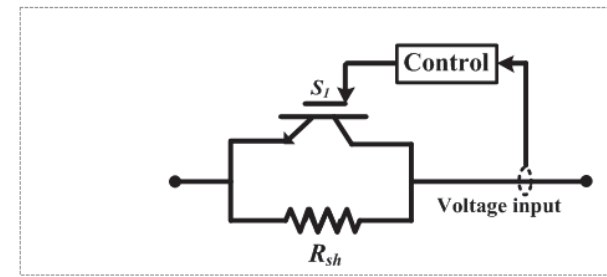

(a)

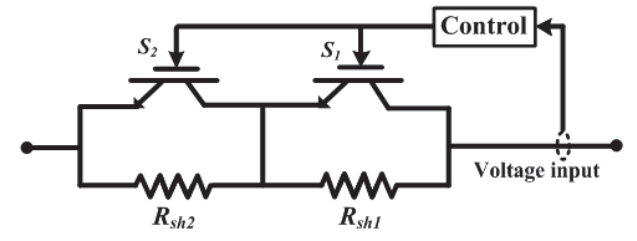

(b)

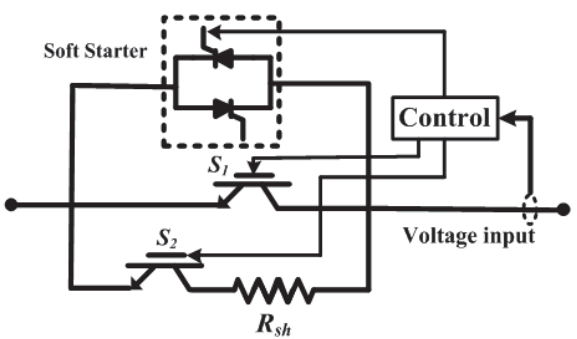

Figure 2.9: Various types of SDBR; (a) Single-stage scheme. (b) Two-stage switching scheme. (c) Variable resistor scheme using soft-starter.

ation in the initial post-fault recovery. Once the voltage recovered above a minimum set point level and met the grid code compliance, the bypass switch is closed and the circuit is returned to its normal state. Figure 9(c) also displays a possible arrangement, using a thyristor based soft-starter that is already utilized for a grid connected FSIG-based wind turbine [112]. Also, ABB represented an additional feature for the SDBR scheme, in which the resistors were independently controlled in each of the three phases, enhancing the scheme's performance during unbalanced fault condition. The effect of SDBR on stator voltage is displayed by the phasor diagram of Figure 2.10, where the stator voltage is increased across SDBR. Since the mechanical torque generated by the induction generator changes with the square of the voltage, the presence of SDBR can increase the mechanical power extracted from the drive train, therefore reducing its rotor speed during a voltage dip. This action can also enhance the post-fault recovery of a WT system. 


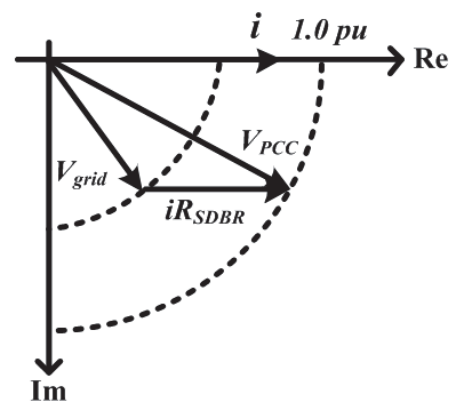

Figure 2.10: Single-phase vector diagram for voltage dip compensation with SDBR.

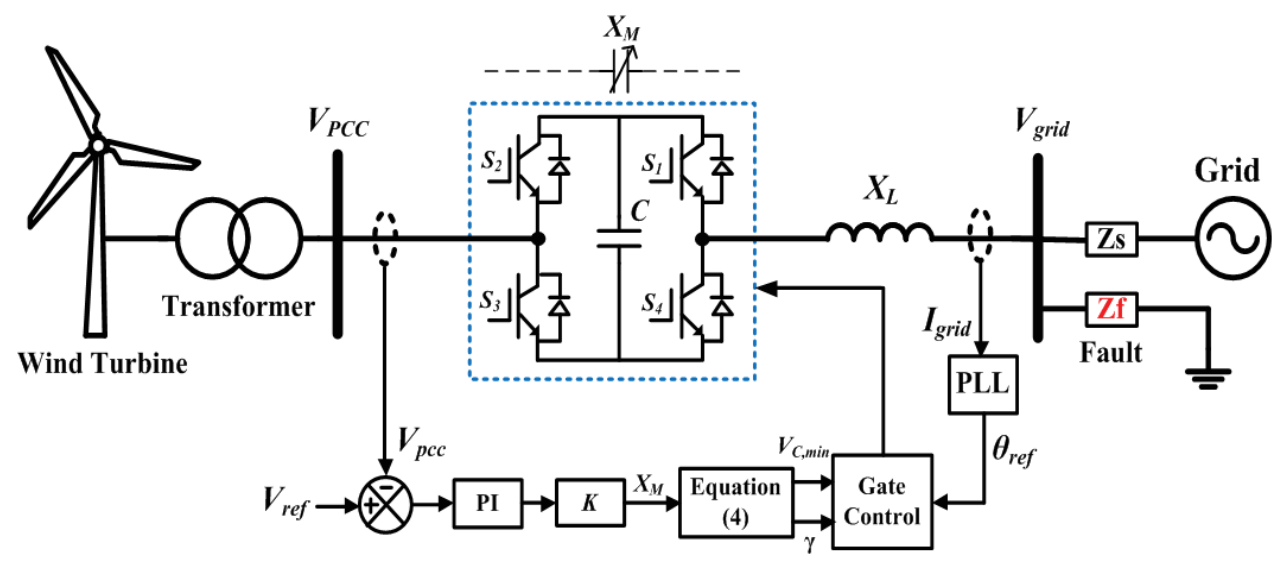

Figure 2.11: Circuit configuration of the MERS for controlling the series-injected voltage.

\subsubsection{Magnetic energy recovery switch (MERS)}

The MERS has recently been proposed as a variable series compensator between the main transformer of the wind farm and the power grid to improve the LVRT capability of fixed-speed WTs by compensating the reactive power and controlling the terminal voltage of the WT [112-115]. The circuit configuration of the MERS is shown in Figure 2.11, including four reverse conductive semi-conductor switches and a dc capacitor. As it is obvious from Figure 2.11, it has a similar topology with respect to a single-phase fullbridge inverter with the exception that dc-link capacitor is several times smaller than that of a single-phase full bridge inverter, due to the capacitor voltage being permitted to alter considerably and to become zero during each fundamental cycle $(50$ or $60 \mathrm{~Hz})$ [115]. Moreover, this scheme possesses fewer losses compared to the PWM converters so that 

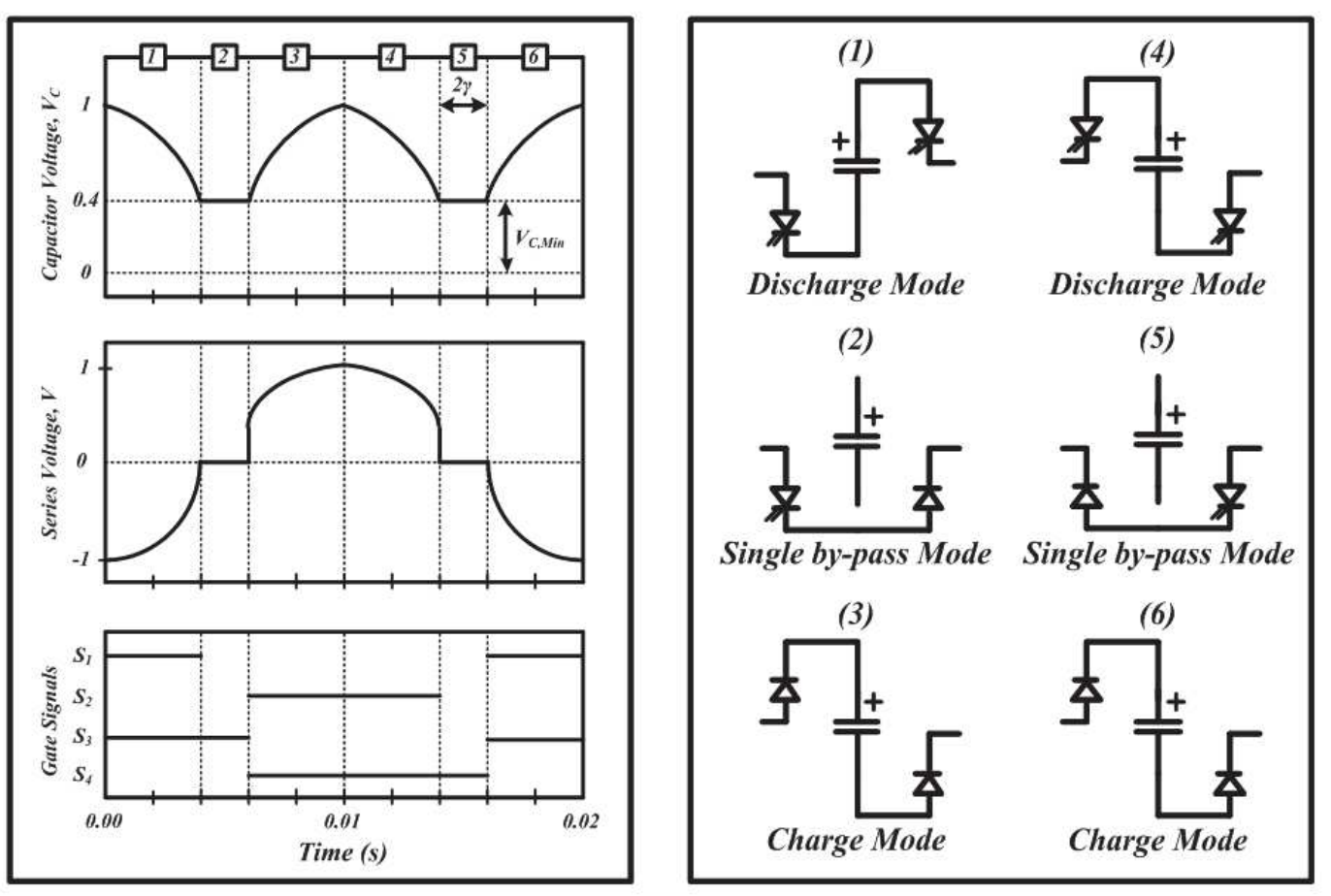

Figure 2.12: Switching patterns for one current cycle. (Left part) voltage and curren$t$ waveform of MERS; (Right part) the flow of the current through the MERS for the different areas illustrated on the left.

semiconductors in MERS are switched synchronously to the line frequency, which is extremely important for high-power wind applications.

The principal results of switching patterns and waveforms for one fundamental cycle are illustrated in Figure 2.12 based on two main set-points control, i.e. minimum capacitor voltage, $V_{C, \min }$ and the length of the zero injected voltage period, $2 \gamma$. By adjusting the $V_{C, \min }$ and the $\gamma$ reference, the current passing through the device can be regulated to provide the variable series-injected from zero to the rated voltage for all currents within the device rating. Wiik et al. developed in [113] a control method suitable for the LVRT application in transmission systems shown in Figure. 2.11 for injecting series voltage based on MERS equivalent compensating reactance expressed as

$$
X_{M}=\frac{1}{\omega C}\left(1-\frac{2 \gamma}{\pi}-\frac{\sin 2 \gamma}{\pi}\right)+\frac{4 V_{C, \min } \cos \gamma}{\sqrt{2} \pi I_{\text {grid }}}
$$




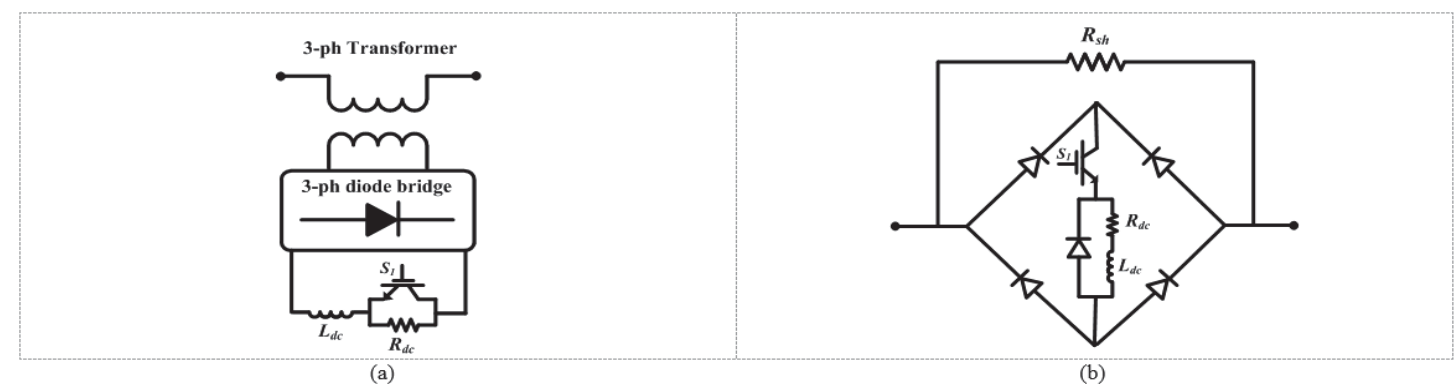

Figure 2.13: Fault current limiter topology. (a) Bridge-type FCL (BFCL). (b) Modified configuration of BFCL.

where, $I_{\text {grid }}$ is the line current and $C$ in the capacitance of the dc capacitor.

\subsubsection{Fault current limiter (FCL)}

The need for FCL is increased by the rising fault current levels due to integration of the high penetration of WTs into the power grids. In recent years, various types of FCL, such as solid state FCL, resonant circuit, transformer coupled bridge-type fault current limiter (BFCL), and superconducting fault current limiter (SFCL), have been proposed and developed $[116,117]$. Previous studies have proven that the ability of SFCL and BFCL technology to improve LVRT capability and enhance the transient stability of the wind generator systems. By using these types of FCL during the fault, the stator current of induction generator has been effectively limited and the voltage reduction level of the generator terminals has been decreased, resulting in fulfilling the international grid codes. Once the FCL is adopted in the WTs system, the peak value of the short circuit current can be limited to a level within the switchgear rating.

\section{Bridge-type fault current limiter (BFCL)}

As shown in Figure 2.13(a) and presented in [118,119], the bridge-type FCL with discharging resistor $\left(R_{d c}\right)$ requires the coupling transformer to be connected to the power grid. A resistor in parallel with a semiconductor switch has been connected in series 
with the dc reactor $\left(L_{d c}\right)$ of the conventional bridge-type FCL in order to control the fault current level by controlling the dc reactor current. The increase of the fault current is curbed by the dc reactor without any delay. This characteristic of the bridge-type FCL suppresses the instantaneous voltage drop and it is able to improve the transient behavior of WTs in the fault instant, which is the main advantage of the bridge-type FCL to other FRT enhancement techniques.

Moreover, $R_{d c}$ in the bridge-type FCL was used to increase the terminal voltage of the generator, thereby smoothing the electrical torque and the active output power fluctuations during the fault. However, this topology needs a special and costly transformer to connect the three-phase diode bridge in series into the system, in which primary voltage rating of the transformer must be almost equal to the transmission line voltage to maintain the desired level of voltage within the fault duration [120].

In [120], the authors proposed a new modified configuration of BFCL, including the four-diode bridge part and shunt resistive path, shown in Figure 2.13(b), in order to achieve the LVRT of fixed speed wind generator system. In normal conditions, the switch must be kept closed, as its gate signal, $S_{1}$ is at a high level, in which a line current through the dc reactor placed within the diode bridge flows in the same direction, charging the $L_{d c}$ to the peak current.

Once the fault occurs, the sudden rise of fault current would be instantaneously limited by the reactor. Hence, abrupt voltage reduction at generator terminal is prevented during the fault, providing the improved transient behavior. Once the line current in dc side $i_{d c}$, exceeds a predefined threshold $i_{t h}$, the IGBT switch must be turned off via sending the low level signal to $S_{1}$. In this case, the diode bridge is cut off and the line current passes through the shunt resistor $R_{s h}$ in order to suppress fault current and consumes excess energy from the wind generator. By controlling the duration of $\mathrm{ON}$ and OFF periods of IGBT switch, control system provides a manageable resistor in order to control the 


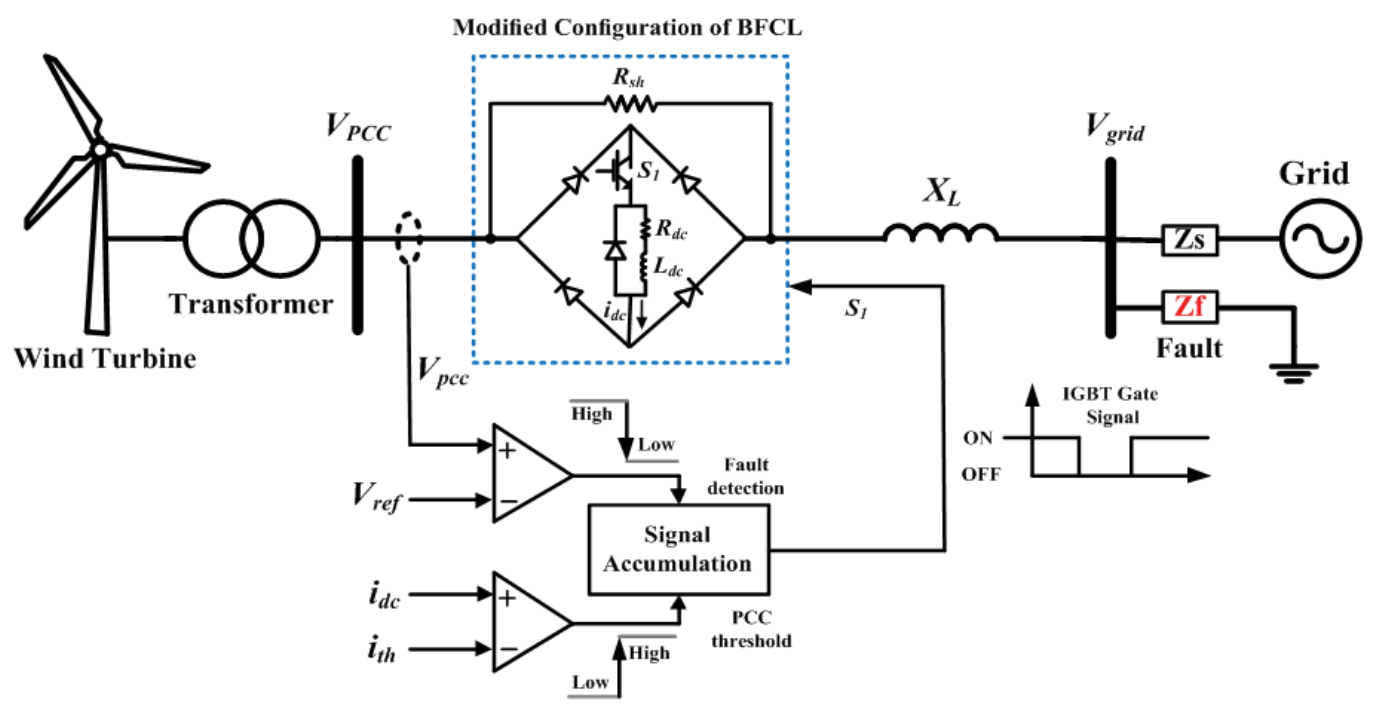

Figure 2.14: Modified configuration of BFCL installed outside the wind farm with the control scheme.

terminal voltage of induction generator, leading to a reduction in the rotor acceleration and stabilizing the system. The controller used for the BFCL was developed in [120] and shown in Figure 2.14.

\section{Superconducting fault current limiter (SFCL)}

The SFCLs have been launched and introduced into the network to restrict the prospective fault currents immediately to a manageable level by suddenly raising the resistance value [21]. SFCL is considered as self-healing technology since it eliminates the need for any control action or human intervention due to its automatic excessive current detecting and the automatic recovering from non-superconducting to superconducting states. By using the SFCL, the fault current is suppressed effectively and the voltage dip level of the WTs terminals is diminished, leading to the enlarging of the voltage safety margin of the LVRT curve $[121,122]$. The first-cycle suppression of a fault current by an SFCL results in an increased transient stability of the power system carrying higher power with greater stability. This innovating device introduces an exclusive feature that cannot be obtained by conventional current limitations. 


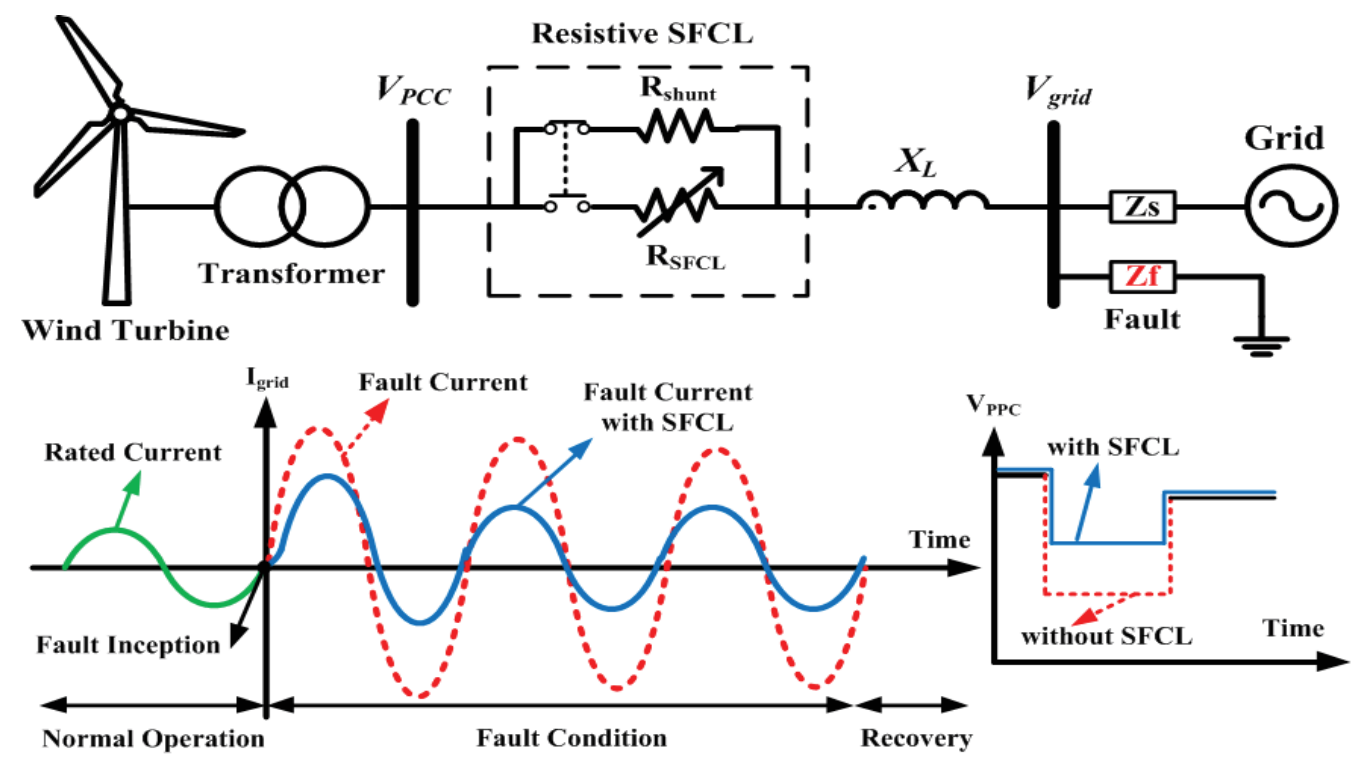

Figure 2.15: Operation of resistive SFCL installed in transmission line including fault current and voltage profile at the wind turbine terminal.

Generally speaking, high temperature superconducting fault current limiters (SFCLs) have been classified into the resistive, inductive, and hybrid types [21]. Amongst diverse SFCL devices, resistive SFCL has a simple structure with a lengthy superconductor wire inserted in series with the transmission lines. To preserve the superconductor from detrimental hot spots during the operation, the shunt resistance, $R_{\text {shunt }}$ is essential. This parallel resistance must be contacted all over the length of the superconductor, and it regulates the controlled current to elude over-voltages likely occurring when the resistance of the superconductor increases much quicker. With the recent breakthrough of economical second-generation high-temperature (HTS) wires, the SFCL has become more viable and is eventually expected to be at least a factor of ten lower in cost than the presently available HTS conductor [43]. The structure of FSIG-based WT with resistive SFCL is schematically shown in Figure 2.15. The current limiting behavior of the RSFCL can be modeled by the resistance transition of HTS tapes in terms of temperature and current 
density as defined by the following equation [122].

$$
\left\{\begin{array}{l}
0 \quad \text { if } \quad J<J_{c} \text { and } T<T_{c} \quad \text { Superconducting state } \\
f\left(\left[\frac{I}{J_{c}}\right]^{n}\right) \quad \text { if } J>J_{c} \text { and } T<T_{c} \quad \text { Flux flow state } \\
f(T) \text { if } T>T_{c}+\text { Normal state }
\end{array}\right.
$$

\subsection{Review on shunt-connected solutions}

Among the external topologies, the shunt-connected devices have been widely utilized to provide smooth and fast steady state and transient voltage control at the point of connection. Since the output current of these devices is adjusted to control either the nodal voltage magnitude or reactive power injected at the voltage terminal, the shunt-connected topology proved to be the most effective solution in the wind power application in order to fulfill the recent international grid codes. A brief explanation of shunt-connected solutions is presented in the following subsections.

\subsubsection{Static var compensator (SVC)}

Thyristor-controlled SVCs, reported in $[123,124]$, have been applied for the voltage support of the critical loads, reactive power compensation, and the transient stability improvement in the electric power transmission systems. The SVC is a combination of a thyristor-controlled reactor (TCR) with a thyristor-switched capacitor (TSC) or MSC as one compensator system, which is practically connected to the PCC bus (or the wind turbine terminals) in order to provide fast voltage support and fulfill LVRT of WTs with induction generators [125-128].

Based on new grid codes, this is a supplementary feature now for wind turbines to supply variable reactive power depending on network demand and actual voltage level, while the crucial problem of SVC is to inject an uncontrollable reactive current dependently on the grid voltage [128]. Thus, the current injected by the SVC reduces linearly with the 


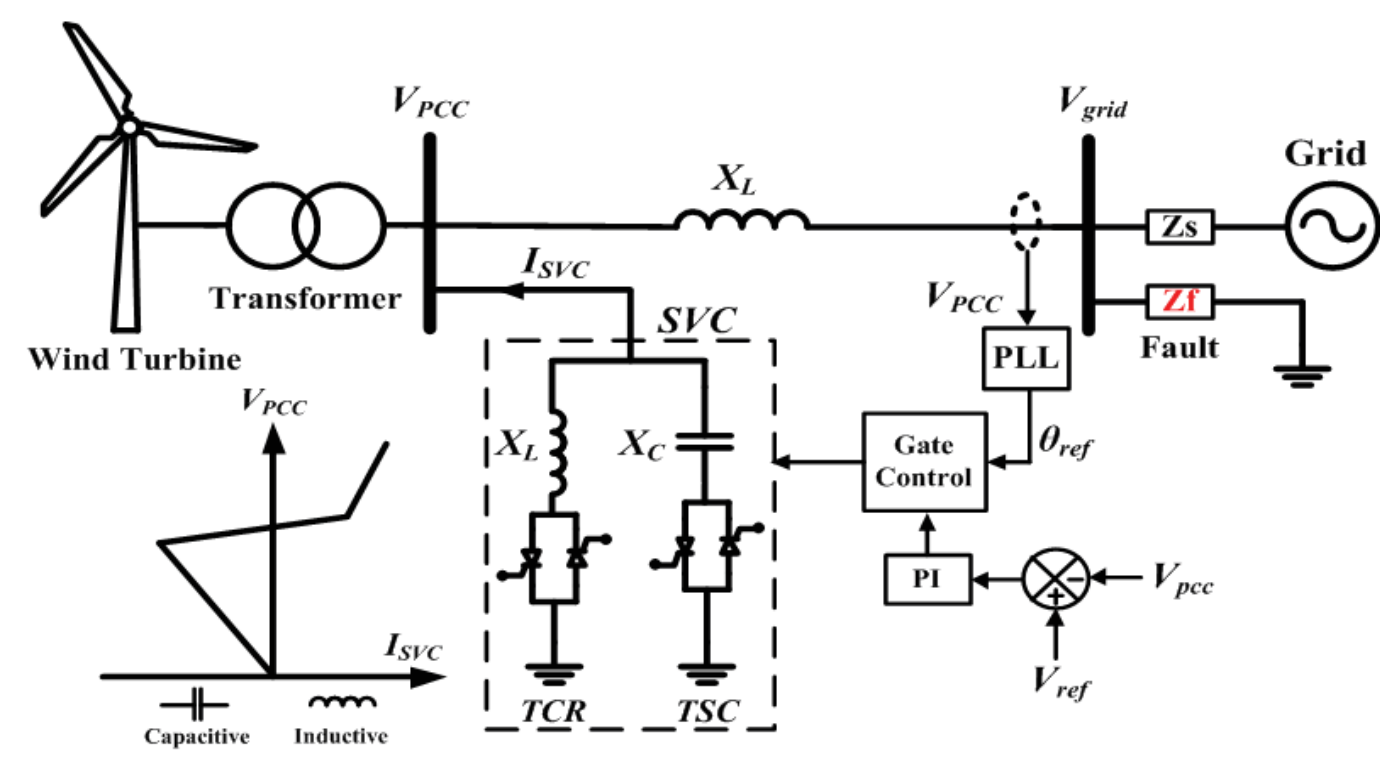

Figure 2.16: Shunt compensation system for wind driven induction generator using SVC along with the basic control system.

voltage sag and consequently the injected reactive power diminishes quadratically. The basic control of the SVC was applied in [128] and shown in Figure 2.16 as a PI controller to control the firing angle of the thyristors of the TCR and TSC, keeping $V_{P C C}$ at 1 p.u during and immediately after the fault. One key issue for designing of an SVC for proper operation is to tune the PI controller, which does not achieve in a simplistic method.

A fast response from the closed loop voltage control of the SVC can cause severe voltage oscillations under week grid operating, in which reduction of transient gain was proposed as a possible solution in order to diminish the SVC's response. However, tuning down the transient gain of SVC leads to a slower voltage recovery after the fault, thereby exceeding the LVRT requirements [129]. In [129], the authors implicitly promoted the idea of using several small distributed SVCs compared to a large central SVC for better voltage response with stable voltage oscillations. A Fuzzy controller was designed in [125] for the SVC to significantly prove an improved dynamic response in terms of overshoot and settling time, as compared to a conventional PI controller. 


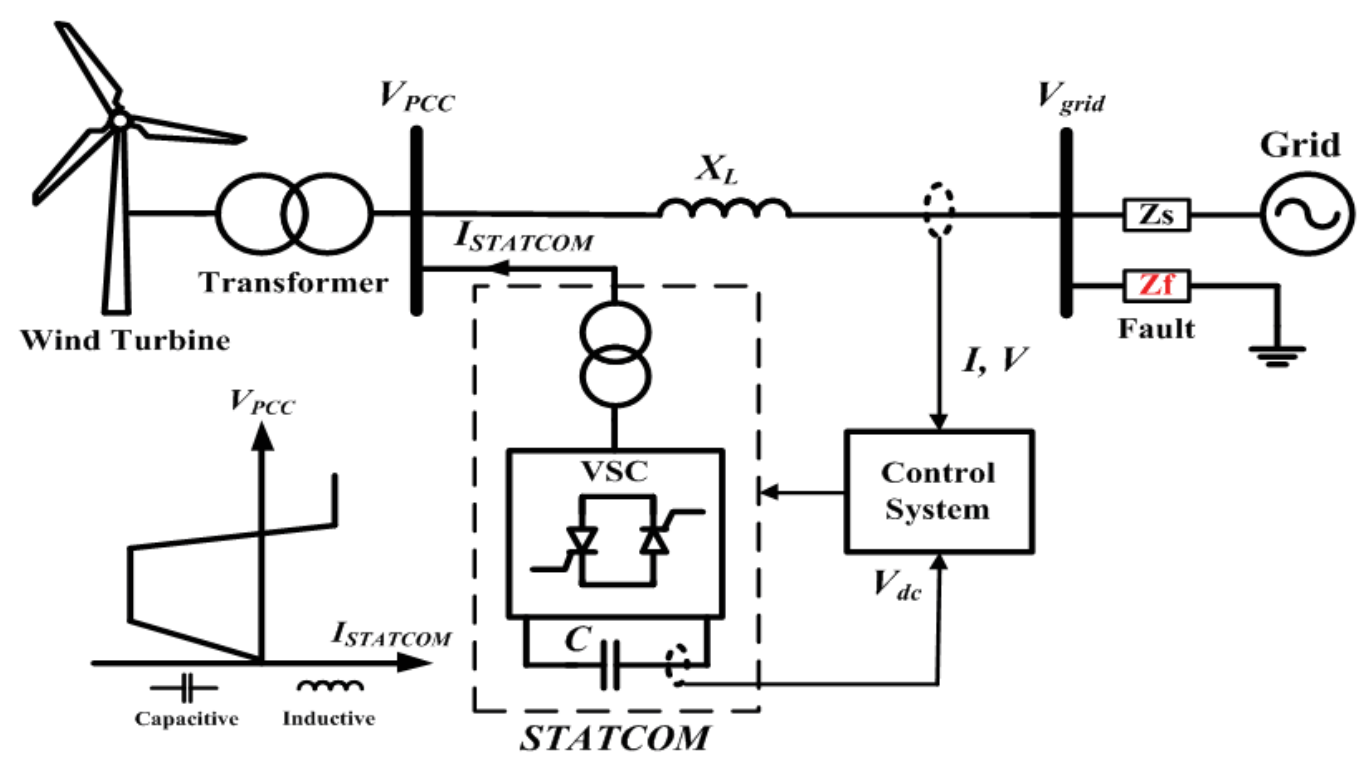

Figure 2.17: The structure of the FSIG-based WT along with STATCOM connected to wind turbine terminal.

\subsubsection{Static synchronous compensator (STATCOM)}

Unlike SVC, the STATCOM, also named SVC Light by ABB [81], can continuously and independently provide a controllable reactive current in response to the voltage reduction, supporting the stability of the grid voltage. The prospect of the STATCOM application in the wind power system emerged in the 1990s, where its significant contribution was power quality improvement during the normal operation. The most important component of STATCOM is the modular voltage source converter (VSC), equipped with insulated gate bipolar transistors (IGBTs), that are controlled by pulse width modulation (PWM). Figure 2.17 displays the basic STATCOM, which can be used in LVRT capability for fixed-speed wind turbines.

STATCOM is connected to the grid to inject or absorb reactive power through a threephase transformer. This system is appropriate to alleviate the effects of both steady-state and transient contingencies [81]. Various efforts have been documented in the literature $[16,128,130-133]$ to prove the ability of STATCOM for the LVRT enhancement of the FSIG-based WT. In [128], Molinas et al. conducted a comparison between the STATCOM 
and SVC in terms of the LVRT improvement. They found that STATCOM could be the economical solution in more situations (15\% cheaper than SVC) if the same rating is assumed for the devices. A modified STATCOM controller was proposed in [16] based on the series combination of a power factor and a voltage regulation loop, which allows an optimized behavior of the fixed-speed WT, both in normal and fault conditions.

The feasibility of incorporating SDBR with STATCOM to fulfill the LVRT requirement of FSWT was investigated in [134], where results showed that the lower STATCOM rating was required compared to utilizing only STATCOM for the same effective performance. Since STATCOM is able to provide only reactive power, application of the energy storage system (ESS) with STATCOM has emerged as a promising solution for the wind power system applications [131]. The new robust decentralized control system for the large interconnected wind power system was introduced in [135] based on the linear quadratic (LQ) output-feedback control method to demonstrate that STATCOM/ESS structure can be an effective device for the grid-code compliant. Another alternative was to simultaneously control both the reactive power and active power via the STATCOM and the pitch angle of the WT to ameliorate the LVRT capability of induction generators in wind farms. It was proved that the combined strategy makes the system ride-through the fault without having to disconnect the generators from the system. However, utilizing the STATCOM for enhancing the LVRT capability augment the torque capability of the induction machine during the recovery process after the fault, causing higher maximum torque, and correspondingly higher stresses on the drive-train. Therefore, authors in [132] suggested a solution based on indirect torque control (ITC) to temporarily set the voltage for the STATCOM controller to limit the maximum torque during the recovery. 


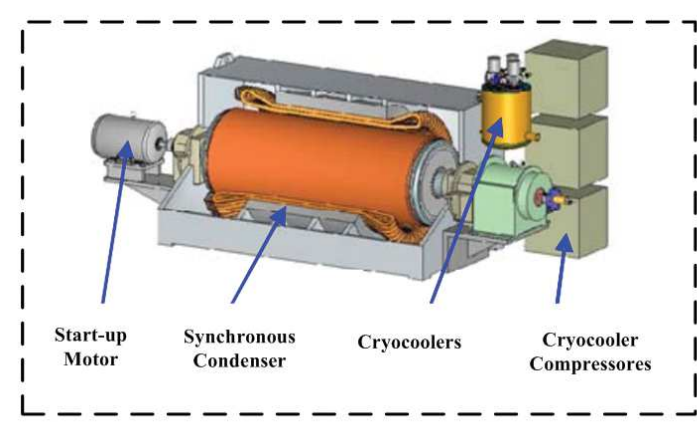

(a)

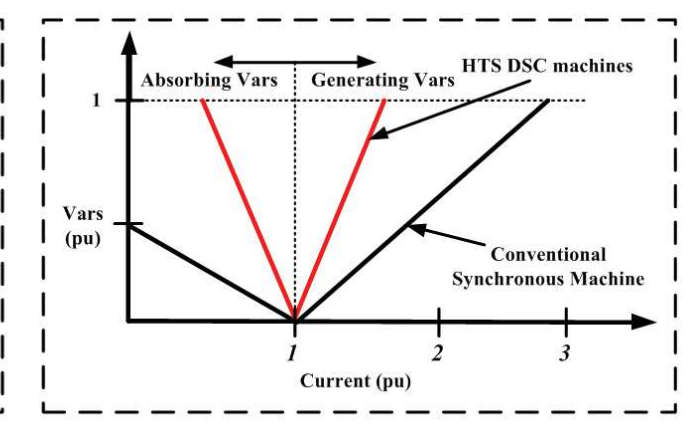

(b)

Figure 2.18: The HTS DSC Concept (a) Structure of the SDSC. (a) Var curve for conventional synchronous and HTS DSC machines.

\subsubsection{Superconducting dynamic synchronous condenser (SDSC)}

One possible solution of integrating large-scale wind farm in the power system comprehensively presented in [136-138], is the superconducting dynamic synchronous condenser (SDSC), shown in Figure 2.18(a), so that rotor windings entailed of HTS wires. Compared with a conventional synchronous condenser, SDSCs provide up to $45 \%$ more dynamic reactive compensation in order to boost the bus voltage during a severe fault situation with power losses and maintenance (Figure 2.18(b)). Since the SDSC machine has a relatively low synchronous reactance relatively low compared with other synchronous machines with the same rating, the machine can respond significantly to transient changes in voltage by injecting or absorbing reactive power. The SDSCs are able to perform with a very high field current (up to 2.0 p.u) for a long period of time, allowing the machine to release the reactive power up to three-times rated output during a transient low-voltage event. Thus, the SDSC can assist a wind farm to meet the interconnection agreement with the utility by providing voltage regulation and improving the stability of a power system [137]. 


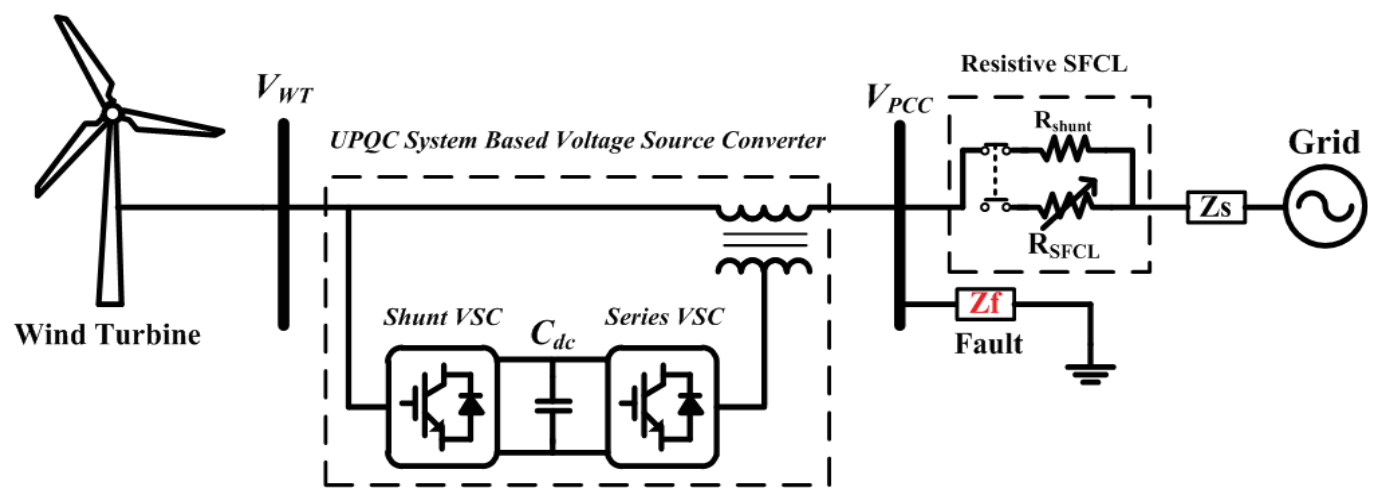

Figure 2.19: The structure of the proposed system: FSIG-based wind turbine with UPQC and SFCL connected to the grid.

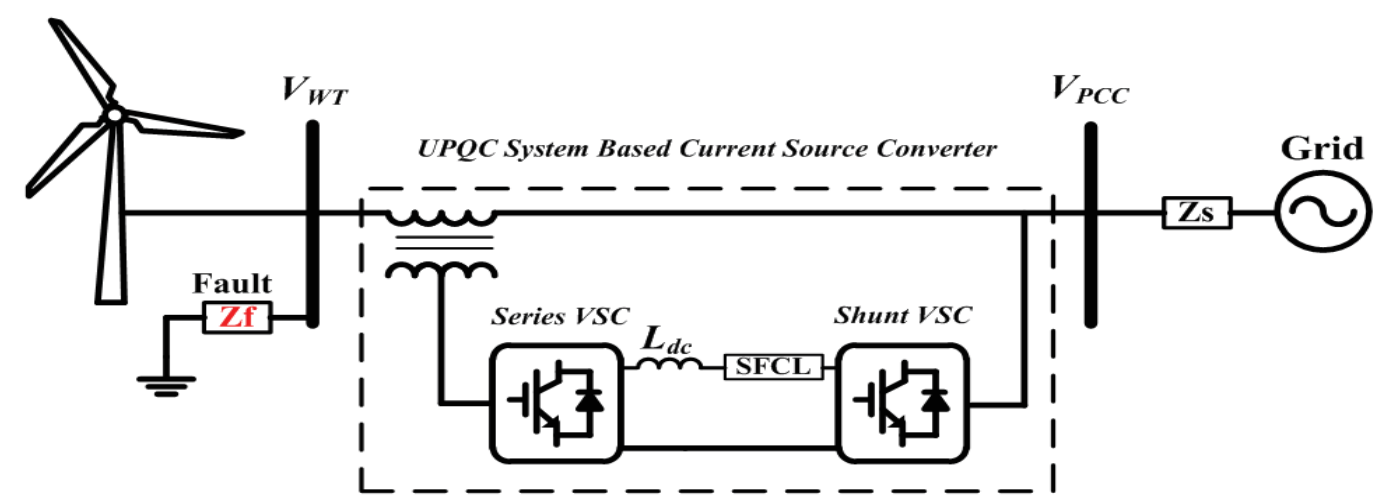

Figure 2.20: The structure of the proposed system: FSIG-based wind turbine with current source UPQC and SFCL connected to the dc link.

\subsection{Review on hybrid-connected solutions}

Reactive power and voltage compensation using series-shunt (hybrid) topologies has been one of the effective techniques in improving the LVRT capability of the large scale of the wind farm level at the point of common coupling. The unified power quality conditioner (UPQC) demonstrates there may be a possible solution to the technical grid integration problems coming from the wind-driven FSIG $[8,9,139]$. Fundamentally, UPQC, which is an integration of series and shunt VSC, has been commonly studied by many researchers as the ultimate device to improve voltage sag, voltage unbalance, harmonics, dynamic active and reactive power regulation [140]. In [139], Jayanti et al. described the application 
of the UPQC systems to enhance low voltage ride-through capability of the FSIG-based wind turbine. The results show that series VSC provides the lack of voltage to prevent over-speeding of the FSIG while the shunt VSC injects additional VAR required during the voltage reduction. However, the capital cost involved in the installation of this device is higher than any other solutions devices because of its use of two converters. Therefore, authors in [8] propose a novel combination of resistive SFCL and UPQC, illustrated in Figure 2.19, in order to improve power quality problems and fulfill grid code requirements. The obtained results confirm that the SFCL cannot only reduce the volt-ampere rating of the UPQC, thereby reducing the installation cost, but also aid the LVRT capability of the wind turbine and improve dynamic performance of the induction generator for additional support.

Moreover, the feasibility of resistive SFCL incorporated in series with the dc-link inductance of the UPQC based on a current-source converter is proposed in [9] to limit excessive current in the event of the generator side fault (see Figure 2.20) and increase the voltage level at the generator terminal, leading to compliance with international grid codes. Huang et al. introduce in [141] a novel topology based on combined shunt and series grid interface configuration, namely, unified compensation system (UCS), to improve FRT capability for FSIG wind turbines. The system structure depicted in Figure 2.21 utilizes one converter to provide both series and shunt compensation. In normal operation, the UCS operates like a STATCOM and supports voltage or reactive power regulation through the shunt connection. In faulty conditions, the UCS instantaneously switches from the shunt to the series grid connection, compensates the voltage, and maintains the stator voltage at its rated value. 


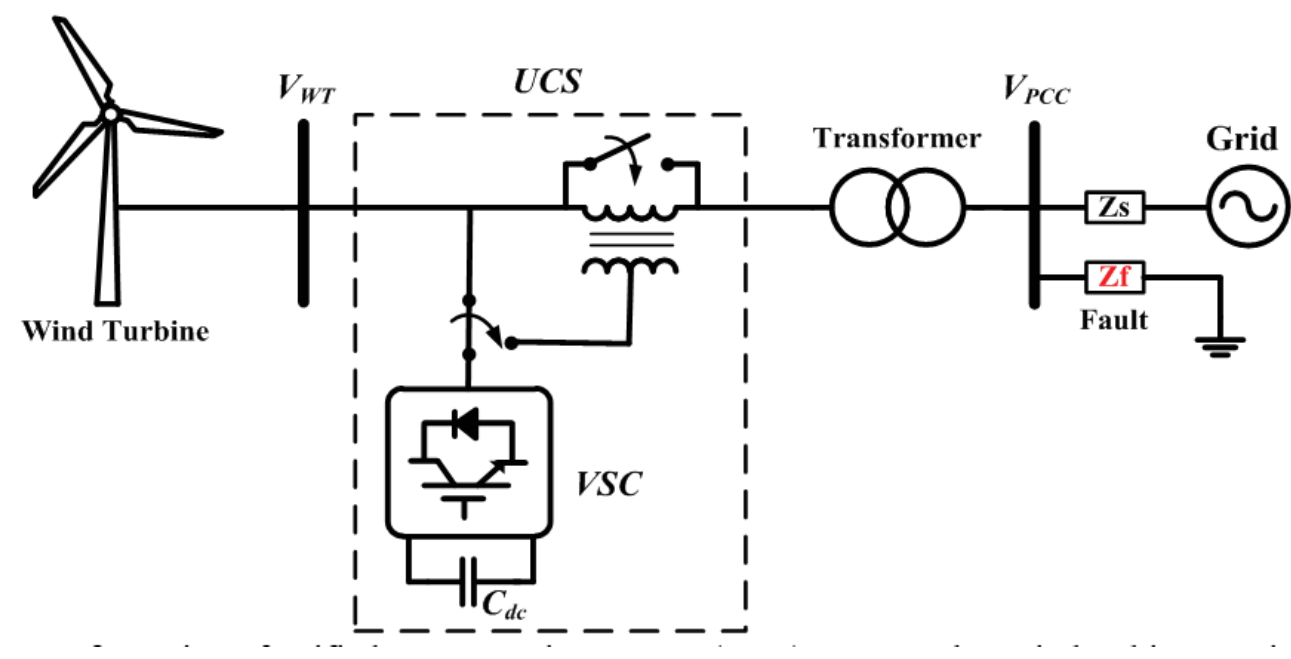

Figure 2.21: Configuration of unified compensation system (UCS) connected to wind turbine terminal.

\subsection{Technical-economical evaluation study of the LVRT methods}

With regard to the above-described LVRT enhancement methods for the FSIG-based WT, no comparative studies have been considered between the different configuration schemes. In this section, a comparative study of these LVRT methods in terms of dynamic performance and economic feasibility is performed.

\subsubsection{Technical comparative study}

Table 2.1 summarizes the advantages and limitations of applying the three categories of the LVRT schemes discussed in Sections 2.3, 2.4, and 2.5. While the final aim of this summary is not to prioritize the LVRT enhancement methods based on technical features, it provides simple and clear metrics, which could be used for decision making purposes.

The contributions of SVC and TCSC to transient voltage stability of the FSIG-based WT and power grid are presented in [102]. The comparative study verified that, in the case of wind speed fluctuation randomly, SVC can offer better reactive power compensation to maintain the transient stability, while TCSC can effectively promote the terminal voltage and enhance the LVRT capability in the case of severe three-phase fault currents. 


\section{Table 2.1: Technical comparison of LVRT improvement methods for FSIG-based WTs.}

\begin{tabular}{|c|c|c|c|}
\hline Methods & Main advantages & Main limitations & Notes(s) \\
\hline TCSC & $\begin{array}{l}\text { 1- Variable capacitive reactance } \\
\text { 2- Useful for voltage unbalance and } \\
\text { fault current limitation }\end{array}$ & $\begin{array}{l}\text { 1- Undesirable resonance } \\
\text { 2- Harmonic injection }\end{array}$ & $\begin{array}{l}\text { 1- An effective solution for offshore } \\
\text { wind farm } \\
\text { - }\end{array}$ \\
\hline DVR & $\begin{array}{l}\text { 1- Fast voltage recovery } \\
\text { 2- Controllable reactive power supply }\end{array}$ & $\begin{array}{l}\text { 1- Phase angle jump } \\
\text { 2- Active power absorption }\end{array}$ & $\begin{array}{l}\text { 1- Compatible with a proper energy } \\
\text { storage capacity } \\
\text { - }\end{array}$ \\
\hline SDBR & $\begin{array}{l}\text { 1- Mechanical active power mitigation } \\
\text { 2- Reduction of pitch angle activation } \\
\text { 3- High reliability and low maintenance }\end{array}$ & $\begin{array}{l}\text { 1- Unable to control reactive power } \\
\text { 2- Unable to damp voltage fluctua- } \\
\text { tions } \\
\text { 3- Useless in low power factor us- } \\
\text { age }\end{array}$ & $\begin{array}{l}\text { 1- } 0.05 \text { p.u SDBR is equivalent to } \\
0.4 \text { p.u of dynamic RPC for LVRT } \\
\text { enhancement } \\
\text { - }\end{array}$ \\
\hline BFCL & $\begin{array}{l}\text { 1-Useful for high voltage drops } \\
\text { 2- Minimizing rotor speed variations } \\
\text { 3- No need to measure any parameters }\end{array}$ & $\begin{array}{l}\text { 1- Needs a large-scale coupling } \\
\text { transformer } \\
\text { 2- Big reactance in huge application } \\
\text { 3- Undesirable saturation of dc re- } \\
\text { actance }\end{array}$ & $\begin{array}{l}\text { 1- Consumes more active power } \\
\text { than the SDBR } \\
\text { 2- Better performance than the SD- } \\
\text { BR in a sudden drop } \\
\text { - }\end{array}$ \\
\hline SFCL & $\begin{array}{l}\text { 1- Automatic fault current detecting } \\
\text { 2- Automatic recovering } \\
\text { 3- Fast fault current limiting action }\end{array}$ & $\begin{array}{l}\text { 1- Unable to work at room temper- } \\
\text { ature } \\
\text { 2- Unable to control reactive power } \\
\text { 3- High recovery time }\end{array}$ & $\begin{array}{l}\text { 1- More feasible with second- } \\
\text { generation of HTS wires } \\
\text { - } \\
\text { - }\end{array}$ \\
\hline SVC & $\begin{array}{l}\text { 1- Reactive current injection } \\
\text { 2- Voltage stability in weak system } \\
\text { 3- Continuous voltage control }\end{array}$ & $\begin{array}{l}\text { 1- Voltage-dependent reactive con- } \\
\text { trol } \\
\text { 2- Unstable voltage oscillations } \\
\text { - }\end{array}$ & $\begin{array}{l}\text { 1- Inject more reactive power com- } \\
\text { pared with SDSC with same capac- } \\
\text { ity } \\
\text { - } \\
\text { - }\end{array}$ \\
\hline STATCOM & $\begin{array}{l}\text { 1- Controllable reactive current } \\
\text { 2- Rapid response to disturbances } \\
\text { 3- Negative-sequence voltage reduction }\end{array}$ & $\begin{array}{l}\text { 1- Needs to cut off in a high voltage } \\
\text { drop } \\
\text { 2- Unable to supply active power } \\
\text { - }\end{array}$ & $\begin{array}{l}\text { 1- Provide faster response com- } \\
\text { pared with SVC } \\
\text { 2- provide less disturbances com- } \\
\text { pared with SVC } \\
\text { - }\end{array}$ \\
\hline SDSC & $\begin{array}{l}\text { 1- Perform with a very high current for } \\
\text { a long time } \\
\text { 2- Low level of losses }\end{array}$ & $\begin{array}{l}\text { 1- Less effective for low voltage } \\
\text { drop } \\
\text { - }\end{array}$ & $\begin{array}{l}\text { 1- Adjust the voltage faster than } \\
\text { SVC } \\
\text { 2- Provide } 45 \% \text { more reactive pow- } \\
\text { er compared to older types }\end{array}$ \\
\hline UPQC & $\begin{array}{l}\text { 1- Both active and reactive control } \\
\text { 2- Fast reactive power compensation } \\
\text { 3- Long critical clearance time }\end{array}$ & $\begin{array}{l}\text { 1- Active power absorption } \\
\text { 2- Needs a huge dc-link capacitor } \\
\text { - }\end{array}$ & $\begin{array}{l}\text { 1- Share voltage control and reac- } \\
\text { tive power control into the two VSC } \\
\text { of the UPQC } \\
\text { - } \\
\text { - }\end{array}$ \\
\hline UCS & $\begin{array}{l}\text { 1- Supporting both shunt and series } \\
\text { compensation with one converter } \\
\text { - }\end{array}$ & $\begin{array}{l}\text { 1- High conduction losses of series } \\
\text { bypass switch } \\
\text { - }\end{array}$ & $\begin{array}{l}\text { 1- Behave like STATCOM in nor- } \\
\text { mal operation } \\
\text { 2- Switch to the series grid interface } \\
\text { in faulty condition }\end{array}$ \\
\hline
\end{tabular}


Although TCSC and SVC have some sophisticated components, such as thyristor, inductors and capacitors, they have a relatively simple control structure.

Compared with SVC, STATCOM provides faster response, fewer disturbances, and better performance at reduced voltage levels; as a result, it is the most extensively proposed solution for the ride-through capability enhancement of wind turbines based on FSIG. STATCOM may be rated for $75 \%$ of SVC rating for the same performance in response to line fault [128]. STATCOM have a slightly smaller footprint than SVC because they use power electronics instead of capacitors and reactors.

In [138], the authors studied a comparison between the SVC and SDSC in terms of voltage regulation in the wind power applications. The results showed that the SVC has better dynamic performance during the fault with minor voltage reduction on the wind turbine terminal, while SDSC could adjust the voltage to the rated value faster during the severe faults, such as three-phase to ground faults.

Among FATCS devices, although UPQC and UCS can exhibit superior performance due to the fact that they provide both series and shunt compensation, the overall cost and control complexity of these types of technologies are higher than other FACTS devices because of using two converters. However, compared with UPQC, UCS has less control complexity because only one converter is connected to the grid at the same time.

The SDBR can be considered a very simple and cost-effective solution for LVRT capability enhancement of the wind generator system since it uses a high power resistor and fewer switches than other auxiliary methods. In [110], the direct comparison of SDBR and RPC for the fixed-speed WT was conducted, and demonstrated that a 0.05 p.u. dynamic resistor is equivalent to 0.4 p.u. of dynamic RPC. However, the SDBR can dissipate active power, but it cannot control reactive power; hence, it is unable to minimize voltage and power fluctuations of wind generator. 
Amongst diverse FCL devices, BFCL and SFCL technologies with simpler control structures have proven their ability to enhance LVRT capability and transient stability of WTs. However, BFCL needs a special and costly transformer to connect the three-phase diode bridge in series into the system.

\subsubsection{Economical comparative study}

This section further provides the economic study of all LVRT solutions to evaluate the complexities and economic feasibility of different existing LVRT methods. Economic considerations take into account the cost of the wind power integration and the cost of allocated auxiliary devices for a range of operating conditions in terms of the cost per $\mathrm{kW}$ or KVar of implementation.

\section{Wind power generation cost}

The installed cost of a commercial wind power project is dominated by the capital cost for the wind turbines, including blades, towers and transformers and this can be in the range of $65 \%$ to $84 \%$ of the total installed cost. The other installed costs of a wind technology can be categorized into three groups, i.e., grid connection costs, including transformers and substations ( $9 \%$ to $14 \%$ ), civil works and construction costs (4\% to $16 \%$ ), and other capital costs, including construction of buildings, control systems and project consultancy with costs share of $4 \%$ to $10 \%$ of the total installed cost. The total installed capital costs for wind technology vary significantly depending on the energy market and the local cost structure. China and India have the lowest installed capital costs for new onshore projects of between USD 1100/kW and USD 1400/kW in 2010 and in the range USD 1850 to USD 2200 in the major developed country markets of the United States, Germany and Spain.

Figure 2.22 presents the assumptions for onshore wind capital costs for typical projects in Europe, North America and China/India for 2010 and 2011, as well as the predicted 


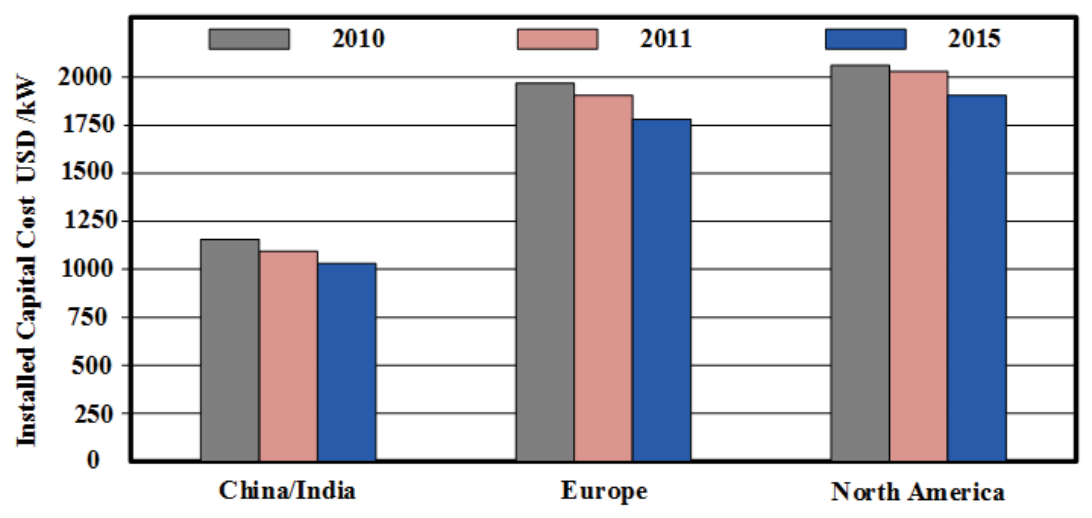

Figure 2.22: Installed cost of wind power projects in three area; 2010, 2011, and 2015.

values for 2015 [142]. Moreover, additional LVRT technologies impact the operation of WT technology economically and technically. Although the actual costs of the auxiliary devices are not widely available, using existing reported data from commissioned projects, the overall cost of these technologies can be roughly estimated. The overall cost of LVRT solutions can be obtained based on their major components, such as number of power electronic switches used, coupling transformer, magnetic inductance, high-power resistance, capacitor, etc.

\section{Economic feasibility of LVRT solutions}

The growing integration of wind generation in power grids is expected to surge the demand of FACTS in different geographies. The overall FACTS market is projected to reach $\$ 1,386.01$ million by 2018 from the $\$ 912.85$ million that it accounted for in 2012 [142]. SVC is the most widely used solution in the global market, followed by the Fixed Series Capacitors (FSC); whereas devices such as STATCOM and UPFC are customized solutions made for the special requirements of the power grids. Obviously, FACTS-based methods are the relatively expensive because they consist of many components, such as the power electronic devices, thyristors, reactors, capacitor banks, switchgear, protection and the control systems, and so on. In this section, the cost range of the major FACTS 


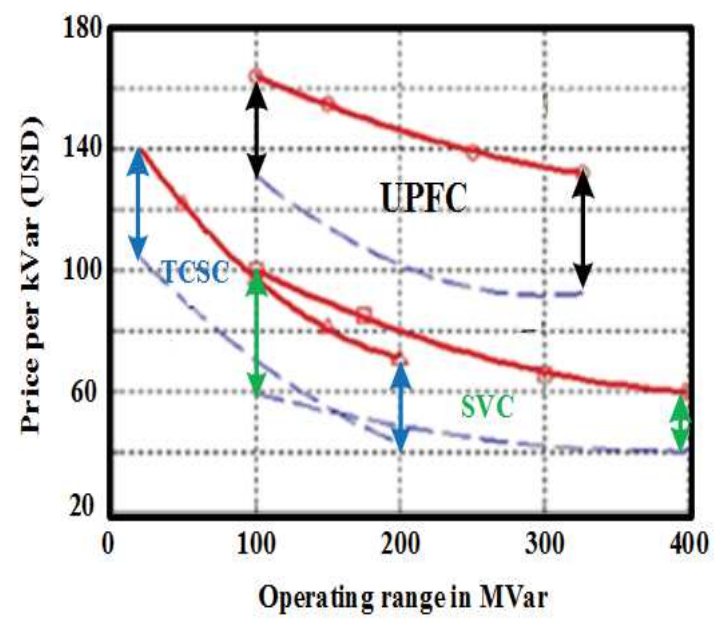

(a)

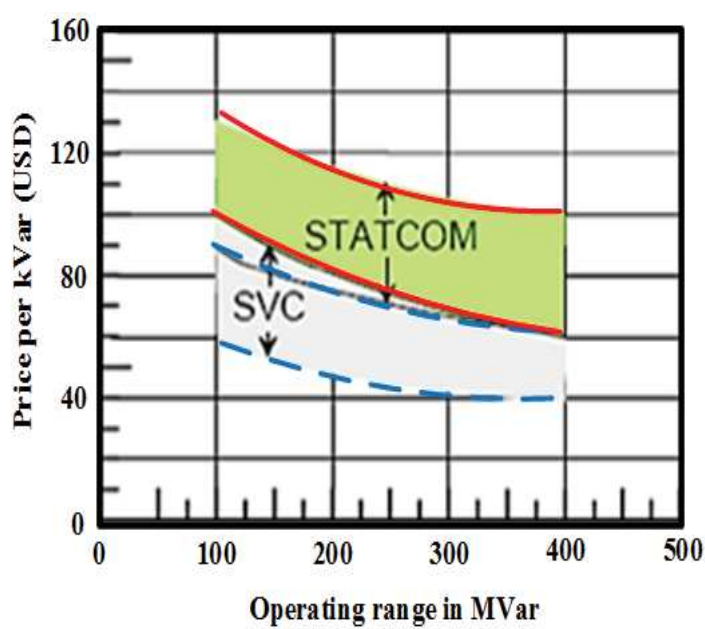

(b)

Figure 2.23: An operating cost comparison between FACTS devices. (a) SVC, TCSC, and UPFC. (b) SVC and STATCOM.

devices is mostly taken from the Siemens and electric power research institute (EPRI) database reported in $[143,144]$, as shown in Figure 2.23(a) and (b).

Accordingly, the cost functions for TCSC, SVC, STATCOM and UPFC are developed as follows:

$$
\left\{\begin{array}{l}
\operatorname{Cost}_{\text {TCSC }}=0.0015 s^{2}-0.713 s+153.75 \quad U S \$ / \text { KVar } \\
\operatorname{Cost}_{S V C}=0.003 s^{2}-0.305 s+128.38 \quad U S \$ / \text { KV } a r \\
\operatorname{Cost}_{S T A T C O M}=0.003 s^{2}-0.233 s+153.45 \quad U S \$ / \text { KV } a r \\
\text { Cost }_{U P F C}=0.003 s^{2}-0.269 s+188.22 \quad U S \$ / \text { KVar }
\end{array}\right.
$$

where $s$ is the operating range of FACTS devices in MVar. The marginal cost per installed $\mathrm{kVar}$ of the FACTS devices decreases as the operating rate capability is increased.

An overall cost for a 100-MVar SVC and a 100-MVar TCSC varies from USD 60 to USD 100 per kVar and USD 70 to USD 95 per kVar, respectively. Although TCSC and SVC have some sophisticated components, such as thyristor, inductors and capacitors, 
they have a relatively simple control structure. Similarly, based on Figure 2.23, the overall cost for STATCOM and UPFC varies from USD 100 to USD 130 per kVar and USD 130 to USD 170 per kVar at 100 MVar rating of the operation, respectively.

A cost analysis has been reported for the DVR in $[145,146]$, where the overall cost including series transformers, VSC using IGBT, and the capacitor bank is estimated between around $\$ 130 / \mathrm{KVar}$ and $\$ 150 / \mathrm{KVA}$ at the operating rate of $100 \mathrm{MVar}$. This research service provides revenue forecasts for the total DVR markets, as well as for low-voltage and medium-voltage restorers. The demand for DVR equipment set the global DVR markets to grow at 6.9 percent between 2004 and 2011, the growth being more prominent in North America and Asia Pacific. The Spanish company CONVERTDIP has successfully put their related products into markets, which are called W2PS [147].

The 8-MVar SDSC machine, developed by American Superconductor, was demonstrated at the Tennessee Valley Authority (TVA) in Gallatin in order to dynamically absorb or produce reactive power, costing between $\$ 1$ million and \$1.2 million [148]. Due to its compact size and the low-cost design, the total cost of the SDSC can be reached up to USD 100/kVar for the operating range of 100 MVar or more. Because of high efficiency and the low maintenance cost of the new HTS dynamic synchronous condenser, it is a very economic option for providing peak and dynamic reactive compensation to a power system. Also, at the present time, the cost of superconducting materials and the cryogenic cooling system of the SFCL are extremely high (up to \$200,000@800 W/2.5kA [149]); thus, to maintain economic feasibility of the final product, the market trend is to minimize the amount of HTS material needed. With the recent breakthrough of economical secondgeneration HTS wires, the SFCL has become more viable and is eventually expected to be at least ten USD less in cost than presently available HTS conductors [150].

The average energy dissipated by SDBR determines its size and cost, so that the power rating of the SDBR is chosen to be greater than average energy dissipated. Once these 


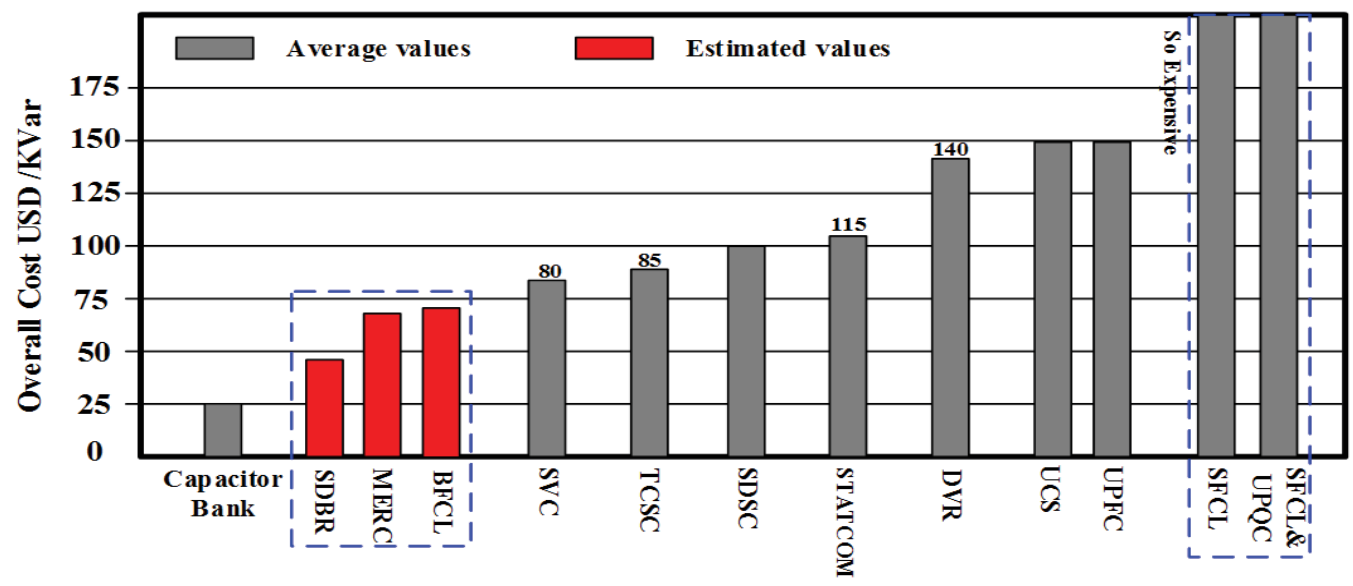

Figure 2.24: Average and estimated overall cost of LVRT solutions for operating range of around 100 MVar.

values are determined, the resistors can be chosen. ABB represented a multi-level structure and called their products Transient Booster. Multistage resistors increase the cost and the complexity of the SDBR, while the single-stage mechanical switching as the lowest cost and least complex option with high reliability and low maintenance and, as a result, single stage SDBR in comparison with FACTS devices may be a preferred solution.

Although cost study of the SDBR, MERC and the BFCL is unreported, based on the complexity of the controller and their configuration, SDBR can be easily considered as the cheapest solution for LVRT improvement after the capacitor bank. The cost of MERC and BFCL can also be estimated as LVRT solutions that are less costly than FACTS devices because they do not require series transformers, sophisticated power electronic converters, or energy storage. As stated in section 2.3.4, the dc-link capacitor of the MERS is several times smaller than that of a regular single-phase full bridge inverter. Thus, between the MERS and the STATCOM with the same topology, the MERC might be cheaper. Since the economical scope of this section is to only compare the average and estimated overall costs of all LVRT solutions for FSIG-based WT, the range of prices (US\$) per KVar is shown in Figure 2.24. 


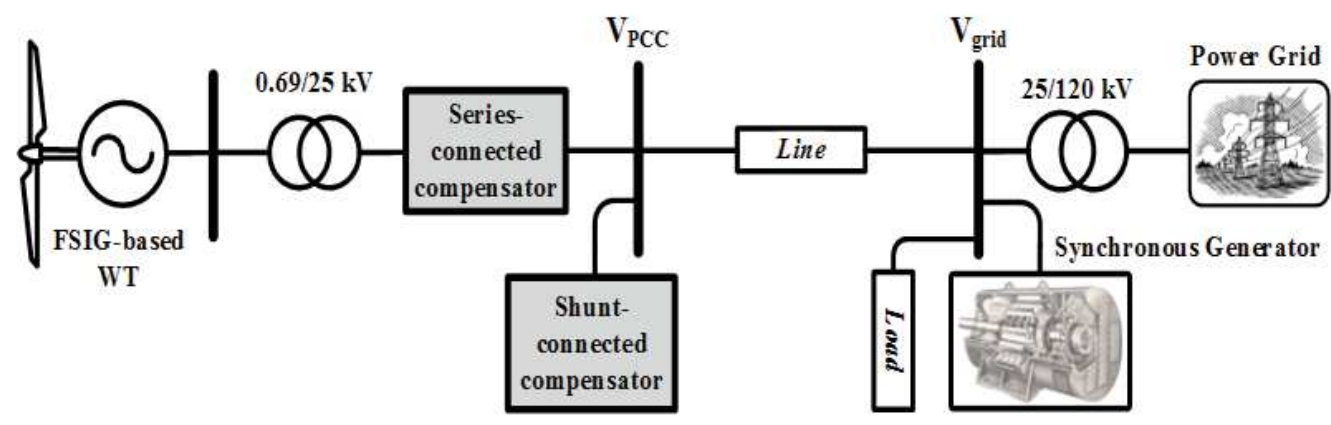

Figure 2.25: Single-line diagram of the power network including a series-connected or shunt-connected compensator.

\subsection{Simulation results and performances comparison}

As stated in Section 2.6 and shown in Table 2.1, the presented LVRT capability enhancement methods for FSIG-based WTs hold some advantages and limitations. To verify effectiveness of the described methods and also to compare them, some simulation studies using MATLAB/SIMULINK software were carried out in this section. The single-line diagram of the proposed power system, including a large wind farm, a hydro power represented by a synchronous generator, and possible shunt and series connected compensators is schematically shown in Figure 2.25. As arbitrary choices, the most common series and shunt connected RPC devices, i.e., STATCOM, SVC, TCSC, and DVR, are applied at the terminal of the wind generator. The parameters of the grid components, FSIG, and RPC devices are given in Tables 2.2 to Table 2.4.

For comparison purposes, the dynamic performance of the combinatorial wind farm and auxiliary devices were compared with the cases without the compensation scheme. A three-phase symmetrical grid fault is considered, since the fault ride-through capability of the regional grid codes mostly refer to this type of fault. Thus, a three-phase fault is applied at $\mathrm{t}=10 \mathrm{~s}$ and is cleared after $150 \mathrm{~ms}$, resulting in $80 \%$ voltage dip at the PCC. The responses of the terminal voltage (PCC), active and reactive power, stator current, and rotor speed of the FSIG are shown in Figures 2.26 to 2.30. 
Table 2.2: FSIG-based WT parameters.

\begin{tabular}{|l||l|l|l|}
\hline Wind turbine Parameters & Values & FSIG Parameters & Values \\
\hline Rated turbine power & $3 \mathrm{MW}$ & Rated power & $3 \mathrm{MW}$ \\
Rated wind speed & $10 \mathrm{~m} / \mathrm{s}$ & Rated voltage & $0.69 \mathrm{kV}$ \\
Blade radius & $44 \mathrm{~m}$ & Stator resistance & $0.0048 \mathrm{pu}$ \\
Optimal power coefficient & 0.45 & Rotor resistance & $0.0044 \mathrm{pu}$ \\
Optimal tip speed ratio & 8.32 & Stator inductance & $0.125 \mathrm{pu}$ \\
Rotor speed & $1.2 \mathrm{p.u}$ & Rotor inductance & $0.179 \mathrm{pu}$ \\
\hline \hline
\end{tabular}

Table 2.3: Synchronous generator parameters.

\begin{tabular}{|l||l|}
\hline Parameters of the grid & Values \\
\hline Rated voltage & $120 \mathrm{kV}$ \\
Rated frequency & $60 \mathrm{~Hz}$ \\
Transmission line & $0.11+\mathrm{j} 0.001 \mathrm{pu}$ \\
Load & $2 \mathrm{MW}$ \\
Rated SG power & $5 \mathrm{MW}$ \\
Rated SG voltage & $25 \mathrm{kV}$ \\
\hline \hline
\end{tabular}

Table 2.4: Parameters of FACTS devices.

\begin{tabular}{|l||l|l|l|}
\hline Parameters of STATCOM & Values & Parameters of TCSC & Values \\
\hline Rated power & $3 \mathrm{MVar}$ & Rated power & $3 \mathrm{MVar}$ \\
Transformer voltage & $2.5 / 25 \mathrm{kV}$ & Rated voltage & $25 \mathrm{kV}$ \\
DC-link voltage & $2760 \mathrm{~V}$ & Capacitance & $21.91 \mathrm{uF}$ \\
DC-link capacitance & $0.02 \mathrm{~F}$ & Reactance & $0.043 \mathrm{H}$ \\
\hline Parameters of SVC & Values & Parameters of DVR & Values \\
\hline Rated power & $3 \mathrm{MVar}$ & Rated power & $3 \mathrm{MVar}$ \\
Transformer voltage & $2.5 / 25 \mathrm{kV}$ & Transformer voltage & $2.5 / 25 \mathrm{kV}$ \\
Rated capacitor power & $3 \mathrm{MVar}$ & DC-link voltage & $2700 \mathrm{~V}$ \\
Rated inductance Power & $1.5 \mathrm{MVar}$ & DC-link capacitor & $6 \mathrm{mF}$ \\
\hline \hline
\end{tabular}

Although all auxiliary devices can meet the LVRT capability requirements of the wind generator, their performance varies with their behavior and capabilities. It is clear that among the described methods, the performances of the STATCOM and DVR are the best and can effectively stabilize the wind generator system, while TCSC exhibits the worst performance; yet it can enhance the LVRT, compared with the "No Compensation" case.

Figure 2.26 shows voltage at the PCC in different methodologies. Among the described methods, the DVR method has the superior performance for diminishing the voltage dip during the fault, where voltage can be significantly retained to around $1 \mathrm{p}$.u, using 


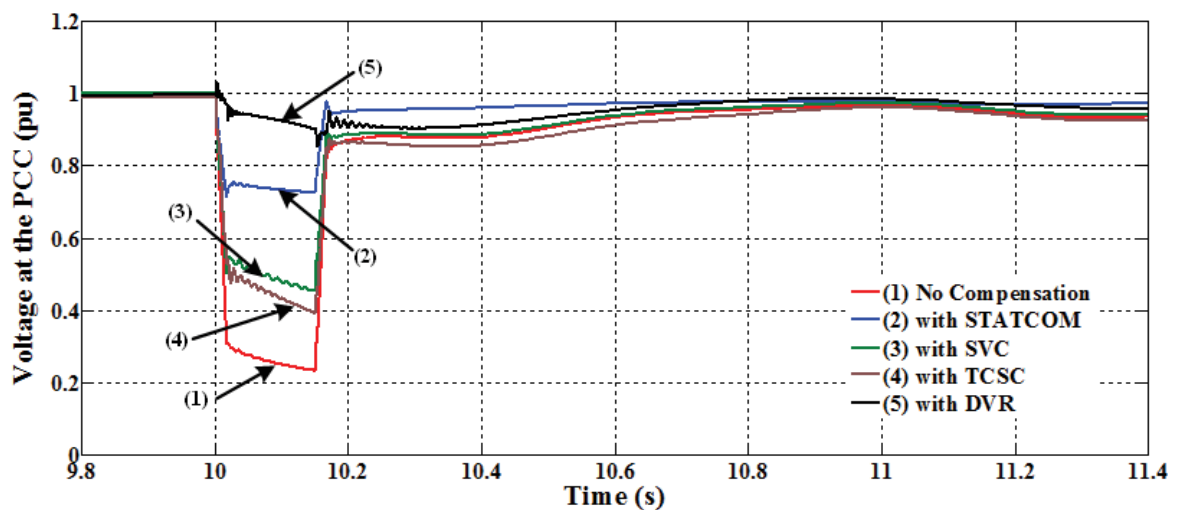

Figure 2.26: FSIG terminal voltage at PCC.

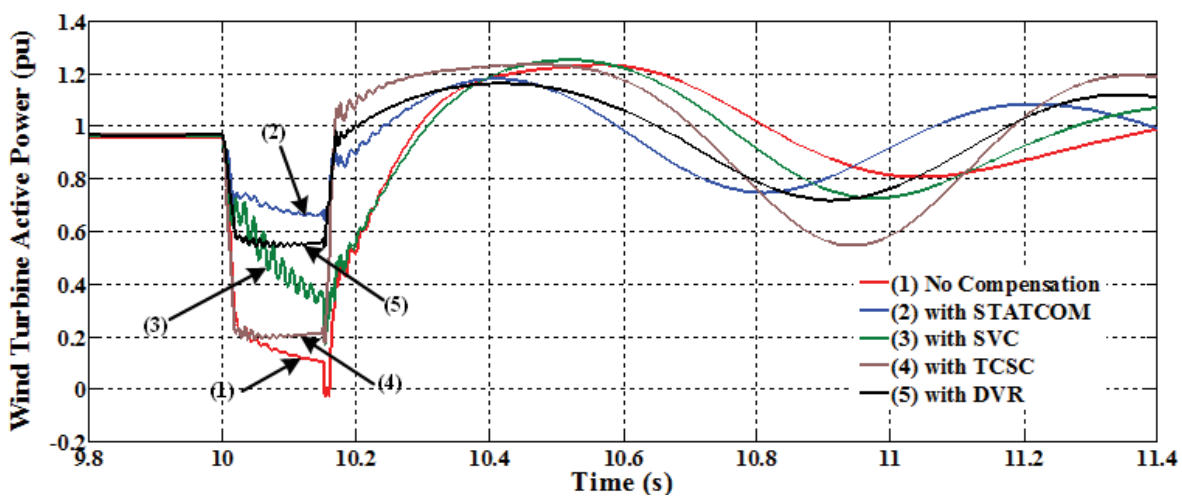

Figure 2.27: FSIG active power output at PCC.

STATCOM after clearing the fault. Since a quick voltage recovery is very important for an FSIG-based WT wind turbine, the STATCOM is a very useful method to provide the quick reactive power and voltage control.

The performance of TCSC and SVC methods are approximately similar and these methods allow wind turbines to handle only a fraction of the total FSIG active power. Figure 2.27 depicts the wind turbine active power during and after clearing the voltage sag. During the fault, the machine output active power becomes almost zero with no compensation. But STATCOM helps maintain more than half of the rated active power at the PCC during the fault. Although the DVR method has the best performance in PCC voltage regulation, when the DVR compensates for the voltage sag; some portion of the 


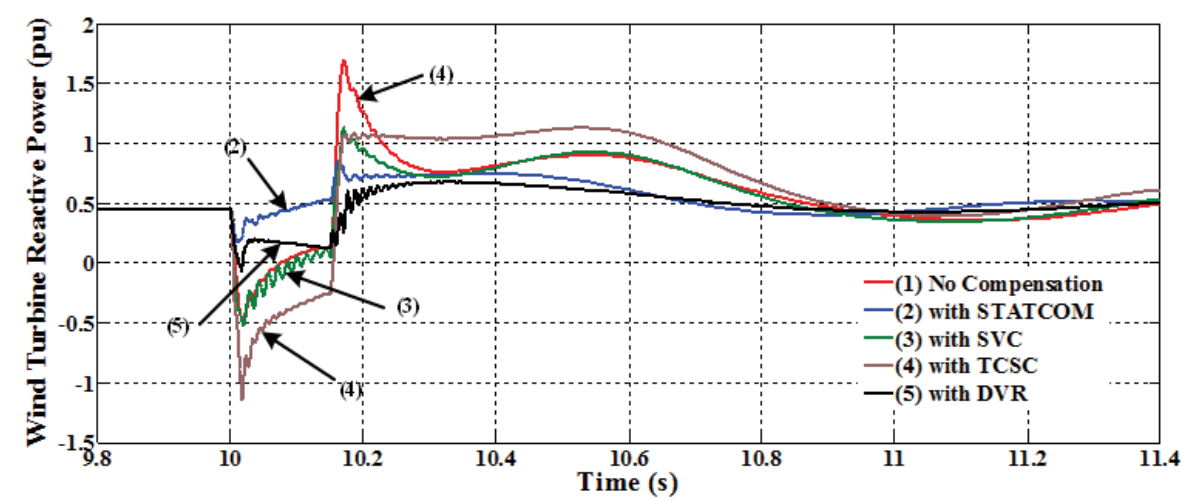

Figure 2.28: Reactive power absorbed by FSIG.

wind turbine active power is partly fed into the DVR system, resulting in lower active power transferred to the grid.

Figure 2.28 illustrates the total reactive power absorbed by the FSIG from the network. After the fault clearing (at $\mathrm{t}=10.15 \mathrm{~s}$ ), the generator needs to draw a large amount of the reactive power to re-establish the magnetic field, which leads to a lower voltage at the terminal of the WT system. However, compared with the no compensation case, the absorbed reactive power from the grid is significantly reduced with the STATCOM and DVR, which helps to avoid other problems, such as voltage collapse and recovery process.

Machine speed response and stator current of the FSIG are shown in Figures 2.29 and 2.30, respectively. As it can be seen, the rotor speed and stator current increase during the fault period, which may lead to a power system instability and it might be detrimental for the turbine generator system if the fault duration is long, and proper auxiliary devices are not used (no controller). Similarly, STATCOM and DVR can limit the rate of rising of machine speed and the magnitude of the machine current in order to make the better stability. 


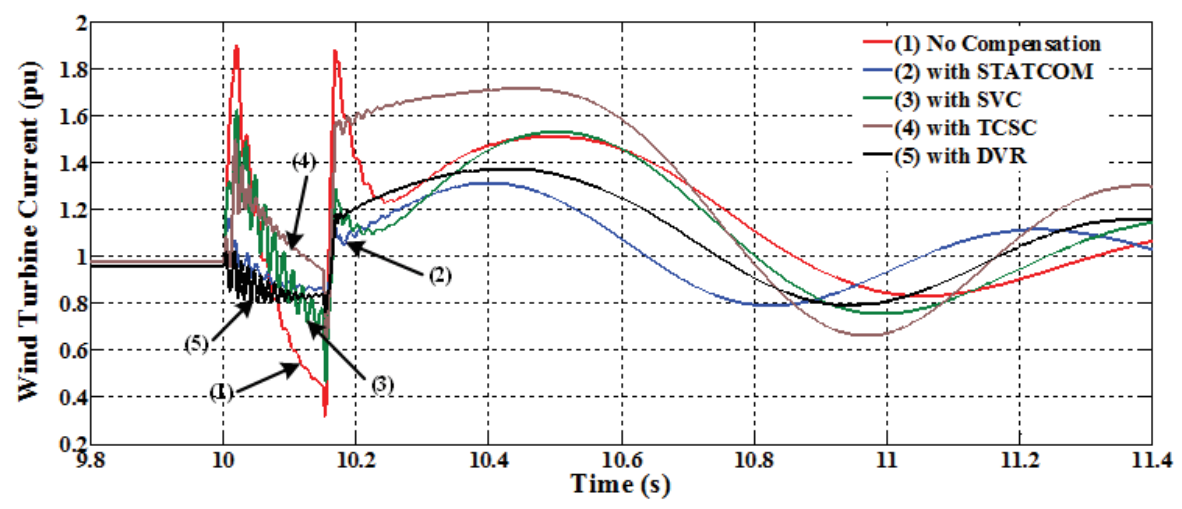

Figure 2.29: Stator current of the FSIG.

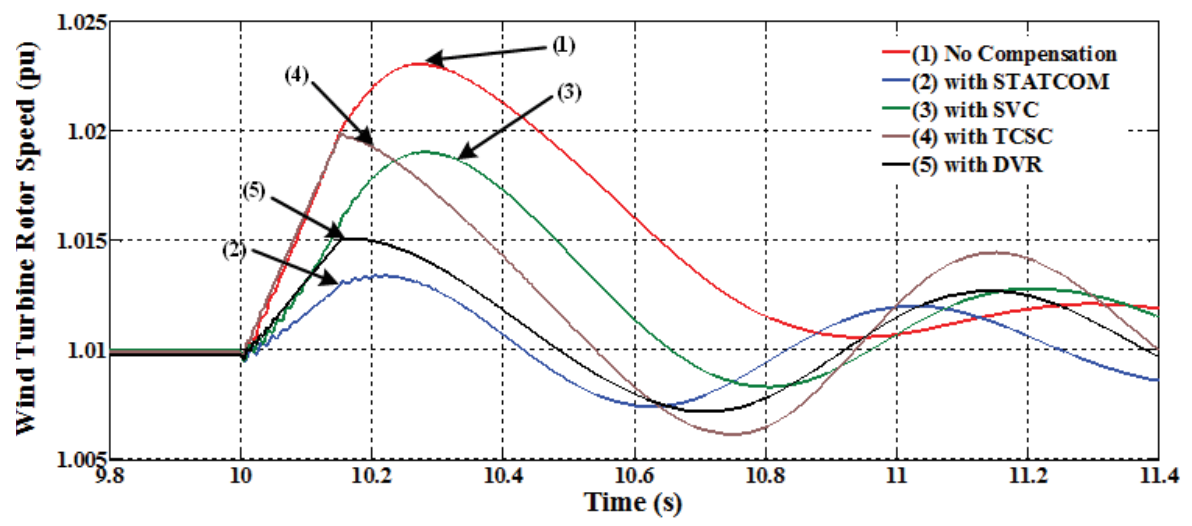

Figure 2.30: FSIG rotor speed.

\subsection{Summary}

This chapter presented the comprehensive review of the state-of-the-art developments for LVRT capability improvement of WTs based on fixed-speed wind turbines, which is relatively a new concept in maintaining the voltage profile of the wind power generation. First, the responses of the FSIG under steady-state and transient-state conditions were extensively discussed. Then, all reviewed methodologies were categorized into three main groups, i.e., series-connected solutions, shunt-connected solutions, and hybrid-connected solutions; discussing the performance of the LVRT schemes including their advantages and limitations in the detail. Also, a comprehensive analysis of these LVRT methods in terms of dynamic performance, controller complexity, and economic feasibility was 
comparatively investigated and summarized in Table 2.1. It is found that the overall cost and control complexity of the SFCL and UPQC schemes are higher than other types of LVRT technologies. On the other hand, the SDBR and BFCL methods were relatively the cheapest and simplest control structure among other LVRT solutions from the economic feasibility point of view.

Finally, some selected case studies were simulated using the MATLAB/Simulink software. Comparison of simulated methods indicated that DVR from series-connected solutions and STATCOM from shunt connected solutions are the most reliable and effective LVRT capability enhancement methods, while TCSC exhibited the worst performance; yet it can enhance the LVRT compared with no LVRT controller employed with FSIGbased WT. Although the market share in the conventional fixed-speed wind turbine concept has diminished, nevertheless a non-negligible $20 \%$ of the existing wind energy in Europe is still employing FSIGs due to their simple structure and lower maintenance cost. Thus, this effort helps the researchers understand the relative effectiveness of the proposed auxiliary equipment and provides a guideline for selecting a suitable technique for the LVRT capability improvement of wind turbine generator systems. 


\section{CHAPTER 3}

\section{APPLICATION OF SUPERCONDUCTIVITY DEVICES ON WIND POWER SYSTEM}

\subsection{Overview}

This chapter is organized as follows. Section 3.2 investigates a fixed-speed induction generator (FSIG)-based wind turbines in an effective combination with a unified power quality conditioner (UPQC) and resistive superconducting fault current limiter (RSFCL). Section 3.3 presents a positive approach using a RSFCL for low voltage ride-through (LVRT) improvement of the permanent magnet synchronous generator (PMSG) based on a large wind power plant (WPP) of 50MW. Section 3.4 presents a combinatorial standalone PMSG based variable speed wind turbine (VSWT) and small-size superconducting magnetic energy storage (SMES) system into the DC microgrid system. Finally, Section 3.5 presents the economic and practical testability of the RSFCL.

\subsection{LVRT Improvement of FSIG WTs Using UPQC and SFCL}

In this section, a cost-effective solution for the FSIG-based wind turbine is proposed to fulfill grid code requirements. This work accomplished by installation of UPQC at the wind farm terminal in order to ameliorate both power quality and LVRT capability of the wind farm. The additional cost to integrate the UPQC can be decreased by utilizing the RSFCL as a self-healing equipment, which is the main motivation of this effort. To ensure the validity of the proposed technique, the whole system is modelled using PSCAD/EMTDC software.

\subsubsection{FSIG-Based Wind turbine}

A significant number of the wind farms in operation are equipped with fixed-speed squirrelcage induction generators and the capacitor banks providing the generator reactive power 


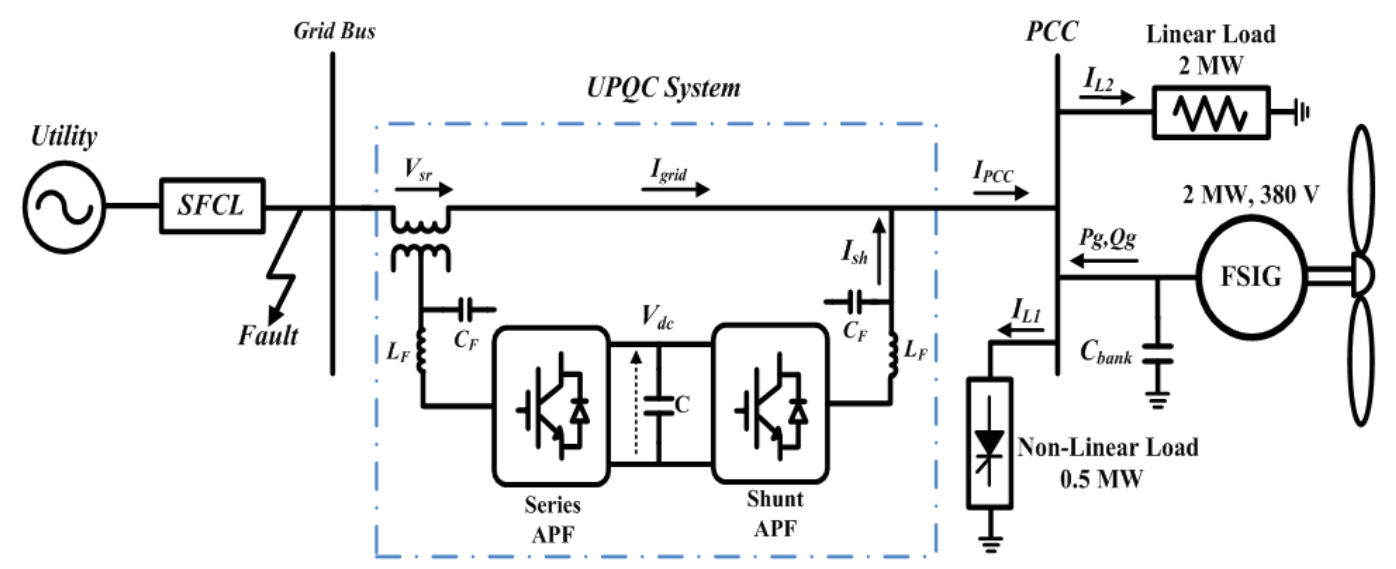

Figure 3.1: Structure of the proposed system: FSIG-based wind turbine with UPQC and SFCL connected to the grid.

requirements. The nonlinear model of the wind turbine is commonly simplified to a static model of the aerodynamic rotor, a two-mass model of the drive train and a third-order model of the induction generator. The general framework of this study is illustrated in Figure 3.1. The mechanical power extracted by a wind turbine from the wind is expressed by the well-known formula

$$
P_{w}=0.5 \rho A v_{w}^{3} C_{p}
$$

where $\rho$ is the air density, $A$ is the area of the rotor disk, $v_{w}$ is the wind speed and $C_{p}$ is the power coefficient. The power coefficient characterizes the rotor aerodynamics as a function of both tip speed ratio and the blade pitch angle. The tip speed ratio is defined as the relationship between rotor blade speed and the wind speed [151]. The drive train of the wind turbine generator is designated by a two-mass model [152], which can be represented by

$$
\left\{\begin{array}{l}
2 H_{r} \frac{d \omega_{r}}{d t}=T_{w t}-T_{m} \\
2 H_{e} \frac{d \omega_{e}}{d t}=T_{m}-T_{e} \\
T_{m}=D_{m}\left(\omega_{r}-\omega_{e}\right)+K_{m} \int\left(\omega_{r}-\omega_{e}\right) d t
\end{array}\right.
$$




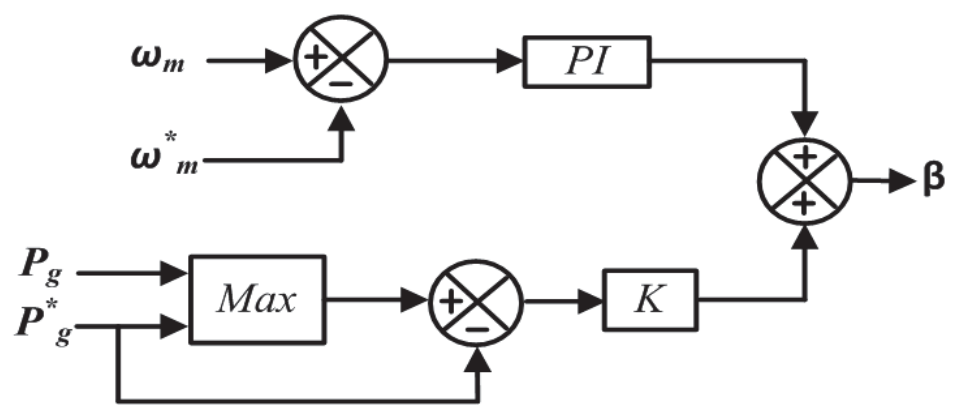

Figure 3.2: Pitch angle Control used in FSIG-based wind turbine.

where $T_{w t}, T_{m}$ and $T_{e}$ are the mechanical torque from the wind turbine rotor shaft, the mechanical torque from the generator shaft and generator electrical torque, respectively. Moreover $H_{r}$ is wind turbine inertia, $H_{e}$ is generator inertia and, $K_{m}$ and $D_{m}$ are the stiffness and damping of mechanical coupling.

Also, in power system stability studies, the third-order model of a FSIG can be achieved by neglecting the stator flux transients in the voltage relations and eliminating the rotor currents. However, the equations used for the squirrel cage induction generator modeling are described in [153]. Also, Figure 3.2 shows the control scheme of the pitch angle regulator for the wind turbine. The inputs to the model are the mechanical speed of the machine, $\omega_{m}$, and the power output of the machine, $P_{g}$.

\subsubsection{Proposed UPQC System}

In this study, the UPQC topology is composed of the integration of a series-active and a shunt-active power filter (APF) connected back to back to a common DC-link, which is recognized as the most complicated power quality improvement, and probably the most expensive one. The major goal of the series APF is to isolate between a sub-transmission system and a distribution system from a harmonic point of view. It is additionally able to compensate voltage imbalance, voltage adjustment and harmonics at the utility-customer at the PCC. On the other hand, the shunt-APF draws current harmonics, compensates for 
reactive power and negative-sequence current inserted by the load. It also controls and manages the DC-link voltage of capacitor to the desired value. The passive filters are also utilized for cancellation of the high switching frequency generated from the shunt and series APFs. Figure 3.1 shows the configuration of the proposed system with UPQC located between the grid bus and the wind turbine terminal.

The series APF operates as a controlled voltage source, keeping the load voltage sinusoidal and at the given constant voltage level. Furthermore, it inserts a voltage equal to the difference of the grid voltage and the nominal load voltage. In order to suppress the harmonic current and compensate the reactive power concurrently, the shunt active power filter is proposed. The operation of the shunt APF is the same as a current source parallel with the non-linear load. In this study, a modified methodology with instantaneous power theory ( $p-q$ theory) is introduced, as an efficacious approach to the non-linear three-phase systems assessment, as well as the shunt APF control [31].

$$
\begin{gathered}
{\left[\begin{array}{l}
p \\
q
\end{array}\right]=\left[\begin{array}{cc}
v_{\alpha} & v_{\beta} \\
-v_{\beta} & v_{\alpha}
\end{array}\right]\left[\begin{array}{c}
i_{\alpha} \\
i_{\beta}
\end{array}\right]} \\
{\left[\begin{array}{c}
i_{\alpha}^{*} \\
i_{\beta}^{*}
\end{array}\right]=\frac{1}{v_{\alpha}^{2}+v_{\beta}^{2}}\left[\begin{array}{cc}
v_{\alpha} & -v_{\beta} \\
v_{\beta} & v_{\alpha}
\end{array}\right]\left[\begin{array}{c}
\tilde{p}-\bar{p}_{\text {loss }} \\
q
\end{array}\right]}
\end{gathered}
$$

The overall control scheme, including series APF and the shunt APF controller, is shown Figure 3.3, where, $V_{P C C}=V_{m} \sin (\omega t+k \pi / 3), k=0,1,2$ is the reference PCC voltage and $V_{F}$ is the generated voltage with series APF. Also, $V_{d c}^{*}$ is the reference value capacitor voltage and $P_{l o s s}$ is converter's switching losses.

\subsubsection{Electro-Thermal Modeling of a Resistive SFCL}

Practically, HTS fault current limiters (FCLs) have been classified into the resistive, inductive, and hybrid types [154-156]. In the three mentioned types of SFCL, the resistive type can protect the system against the fault current instantaneously by a considerable 


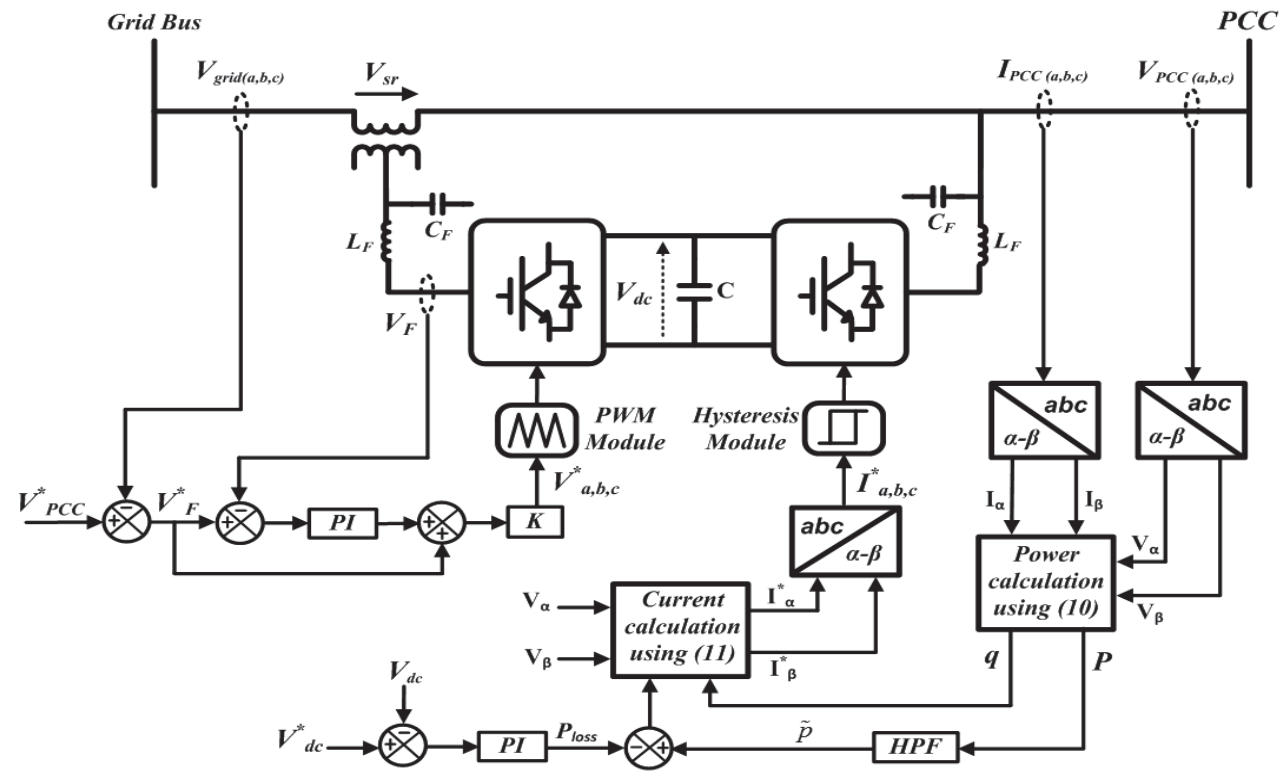

Figure 3.3: Proposed control structure of the UPQC to compensate voltage sag and current harmonic.

increment in the resistance. This type of SFCL is not structurally intricate. Resistive type SFCL also can be switched to the normal operation condition autonomously after barricading the fault current. Hence, the damping performance of RSFCL is recognized as multi-sided cooperation between the fault current magnitude values, thermal properties, and the variable resistance of the HTS substrate.

The RSFCL is based on superconducting bars or double non-inductive spirals for effective elimination of inductance [155]. YBCO-coated conductors are generally used for the resistive type of SFCLs. The current limiting behavior of the RSFCL can be modeled by the resistance transition of HTS tapes in terms of temperature and current density, as defined by the following equation.

$$
R_{S F C L}= \begin{cases}0 & \text { if } i_{s c}<I_{c} \text { and } T<T_{c} \\ \rho_{f} \frac{V_{s c}}{A_{s c}^{2}}\left\{\frac{J_{c 0}}{|J|}\left(\frac{T-T_{c}}{T_{c}-T_{b}}\right)+1\right\} & \text { if } \quad i_{s c}>I_{c} \text { and } T<T_{c} \\ \rho_{n} \frac{V_{s c}}{A_{s c}^{2}}\left(\frac{T}{T_{c}}\right) & \text { if } T>T_{c}\end{cases}
$$


where $T_{c}, T_{b}, J_{c 0}, V_{s c}$, and $A_{s c}$ are critical temperature, liquid nitrogen temperature, critical current density, superconductor volume, and cross section, respectively. Also, $i_{s c}, I_{c}$, $\rho_{f}$, and $\rho_{n}$ are short-circuit current, critical current, flux flow resistivity, and normal resistivity, respectively. In Equation (3.5), three possible states for the superconductor are; 1) the flux-creep state at a temperature and a current under the critical rate; 2) the flux flow state at a current over the critical value, but temperature under the critical rate; and 3) the normal conductive state at a temperature higher than the critical amount.

\subsubsection{Numerical Simulation Analysis}

In this section, in order to verify the effectiveness of the proposed structure shown in Figure 3.1, simulation studies were carried out using PSCAD/EMTDC. In the simulation, by utilizing the proposed structure, the LVRT capability and dynamic stability of the FSIGbased WT is enhanced. A 2-MW FSIG is directly connected to the PCC. For the reactive power compensation purpose, a capacitor bank $\left(C_{\text {bank }}\right)$ is connected to the terminal of the induction generator. The value of the capacitor is chosen so that the power factor of the wind generator during the rated operation becomes unity.

The total output power of a wind turbine is supplied to the resistive load ( $\left.L_{2}, 2 \mathrm{MW}\right)$ installed at the PCC. Moreover, the nonlinear and sensitive load of a three-phase diode bridge $\left(L_{1}, 50 \mathrm{kVA}\right)$ supplied by the grid was considered to affirm the ability of UPQC in reducing current harmonics. Finally, the grid is represented as an infinite source with the fixed frequency of $50 \mathrm{~Hz}$ and voltage of $380 \mathrm{~V}$ interconnected to the infinite bus via the RSFCL. Tables 3.1 and 3.2 show wind turbine characteristics and RSFCL parameters, respectively. The detailed information about UPQC parameters can be found in [21], in

which supply voltage is $380 \mathrm{~V}$, and the resistance and inductances of the system are 0.006 $\Omega$ and $0.02 \mathrm{mH}$, respectively. 
Table 3.1: Characteristics of the wind farm induction generator.

\begin{tabular}{|l||l|l|}
\hline Symbol & Quantity & Value \\
\hline$P_{\text {rated }}$ & Rated generator power & $2 \mathrm{MW}$ \\
$V_{r m s}$ & Rated rms line-line voltage & $0.38 \mathrm{kV}$ \\
$R_{s}$ & Stator resistance & $0.066 \mathrm{pu}$ \\
$R_{r}$ & Rotor resistance & $0.103 \mathrm{pu}$ \\
$C_{\text {bank }}$ & Compensation capacitor & $0.9 \mathrm{MVar}$ \\
$P$ & Poles pairs & 3 \\
$H$ & Mechanical time constant & $2.5 \mathrm{sec}$ \\
\hline
\end{tabular}

Table 3.2: Parameters of SFCL.

\begin{tabular}{|l||l|l|}
\hline Symbol & Quantity & Value \\
\hline$T_{c}$ & Critical temperature for HTS & $99^{0} \mathrm{~K}$ \\
$T_{0}$ & Temperature of cooling system & $77^{0} \mathrm{~K}$ \\
$I_{c 0}$ & Critical current & $5 \mathrm{kA}$ \\
$C_{p}$ & Specific heat of HTS & $3 \mathrm{MJ} m^{-3} K^{-1}$ \\
$P_{c o o l}$ & Power of cooling & $0.9 \mathrm{MVar}$ \\
$V_{s c}$ & HTS Volume & $2 \mathrm{e}-3 m^{3}$ \\
$A_{s c}$ & HTS Cross section & $3 \mathrm{e}-5 m^{2}$ \\
$\rho_{n}$ & Normal resistivity & $4 \mathrm{e}-9 \Omega m$ \\
$\rho_{f}$ & Flux flow resistivity & $1 \mathrm{e}-10 \Omega m$ \\
\hline \hline
\end{tabular}

Figure 3.4 shows the current waveform of: 1) the load and the grid and 2) the shunt active filter, in which the load current can be compensated by the shunt active filter current, keeping the grid current sinusoidal. To assess the operating characteristic and damping performance of the RSFCL, simulations based on the sample parameters are carried out for two scenarios: 1) without considering the SFCL, 2) with considering the SFCL. A symmetrical fault was considered at the integration point with the grid, as illustrated in Figure 3.1. The fault occurs at $1.1 \mathrm{~s}$ and cleared after $0.2 \mathrm{~s}$. For the results in this section, the wind turbine operates at a wind speed of $13 \mathrm{~m} / \mathrm{s}$. In the absence of the SFCL, voltage sag of 50\% occurs at grid bus. Figure 3.5(a) shows the grid voltage and voltage injected by the series active filter and Figure 3.5(b) shows the PCC voltage, which is kept in 1 pu.

The expediency of the SFCL component for managing the fault current, as well as resistance and temperature variations of the SFCL, is demonstrated in Figure 3.6. Before 


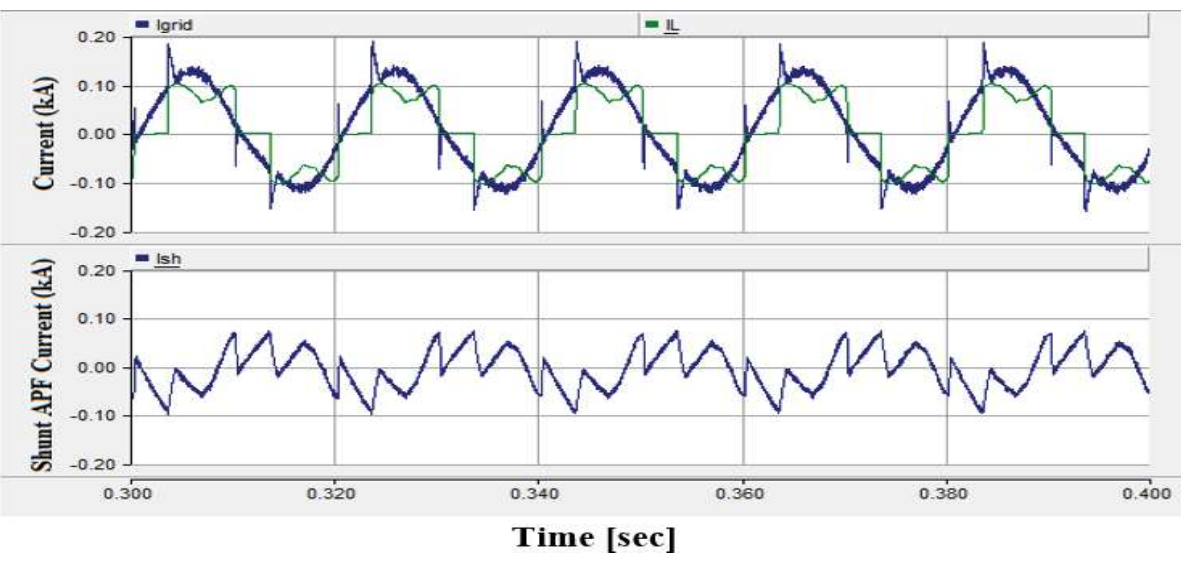

Figure 3.4: Current harmonics compensation. (a) Load and grid current. (b) Shunt active filter current.

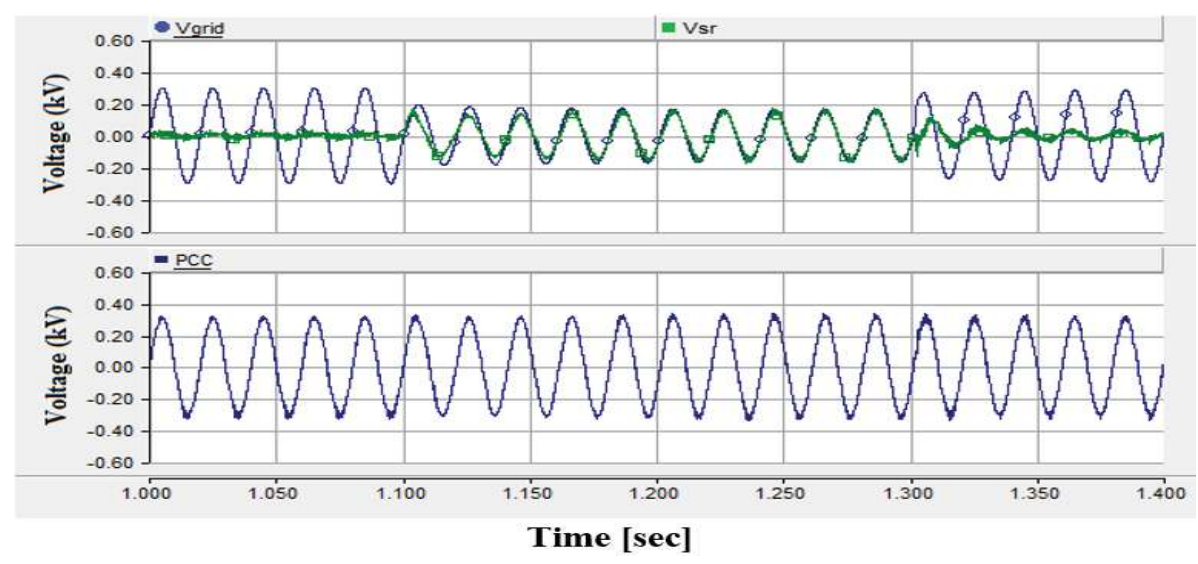

Figure 3.5: Voltage sags compensation without SFCL. (a) Grid voltage and series APF voltage. (b) PCC voltage with compensation.

connecting the SFCL, the peak of the current signal reaches about $14 \mathrm{kA}$ for phase A. The installation of the SFCL on the main road of the wind farm can limit the maximum fault current effectively to reach about $10 \mathrm{kA}$ in the first peak, and was further reduced to $6 \mathrm{kA}$ in the third cycle. A retrieval of Figure 3.6(b) and 3.6(c) will determine when a fault takes place at $\mathrm{t}=1.1 \mathrm{~s}$, the quench time (a transition from promising its superconducting mode to a resistive mode) is initiated by going through the flux-flow state during $0.1 \mathrm{~s}$ and then to the normal state at a temperature rise of $90^{\circ} \mathrm{K}$ (critical temperature for HTS tap). Notwithstanding, this temperature rise requires around $0.4 \mathrm{~s}$ (20 cycles) for the HTS 


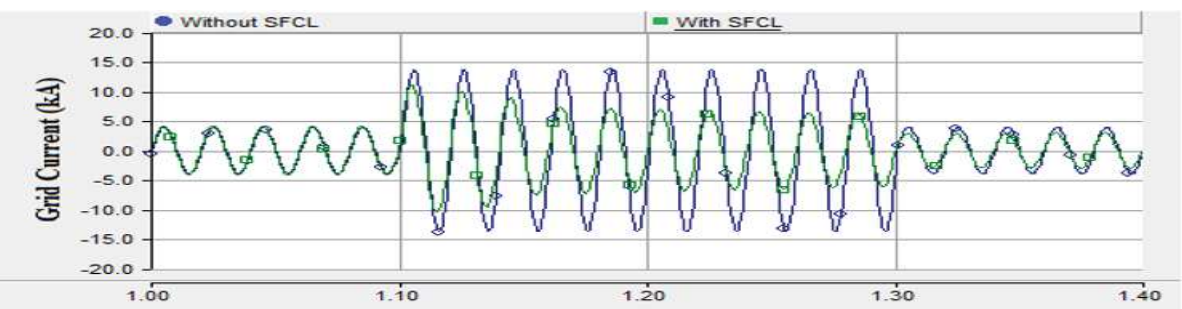

(a)

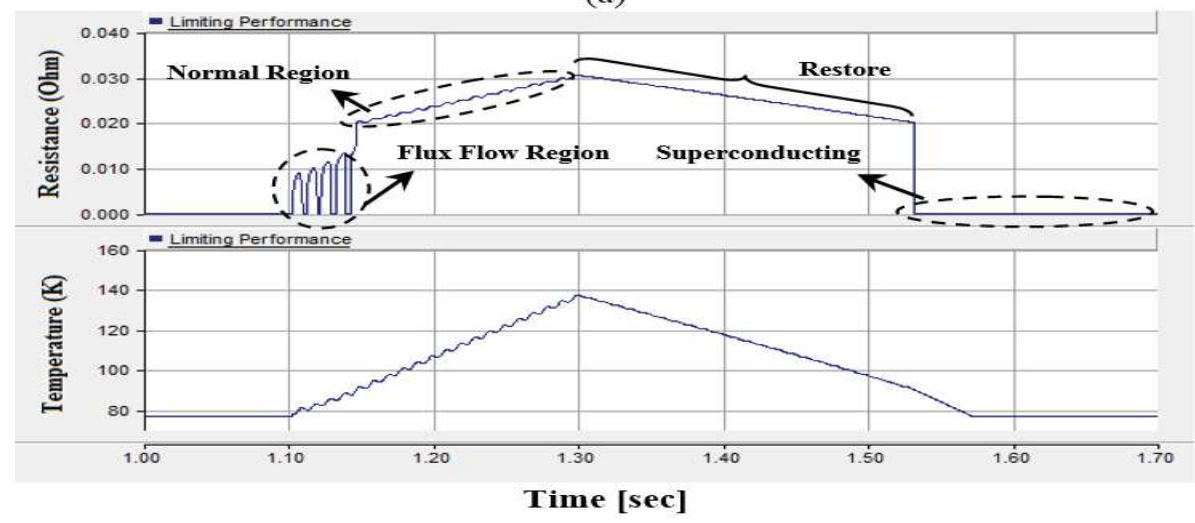

(b)

Figure 3.6: Performance evaluation of the SFCL model. (a) Fault current without and with SFCL in a single-phase system. (b) Resistance variation and temperature rise.

to reverse to its superconducting region, called restore time. Additionally, the limiting resistance of the SFCL went up to $0.003 \Omega$ in the flux flow state and reached its normal state value of $0.023 \Omega$ after ten cycles of the fault.

Figure 3.7 shows the evolution of voltages at the wind turbine terminal with and without the SFCL. The impact of the installed SFCL on the voltage profile was significantly demonstrated by comparison of the voltage waveforms. The voltage sag was efficaciously improved by adding the SFCL, reaching $35 \%$ and $20 \%$ during the flux flow and normal state, respectively. It was observed that the PCC voltages recovered immediately to the nominal value upon clearing the fault.

Figure 3.8(a) shows the grid voltage and voltage injected by the series active filter and Figure 3.8(b) shows the PCC voltage, which is kept in 1 pu with SFCL. Figure 3.9 shows a section of the simulated output active and reactive power with and without connecting SFCL. Before fault occurs, active power delivered to the grid is kept constant at the rated 


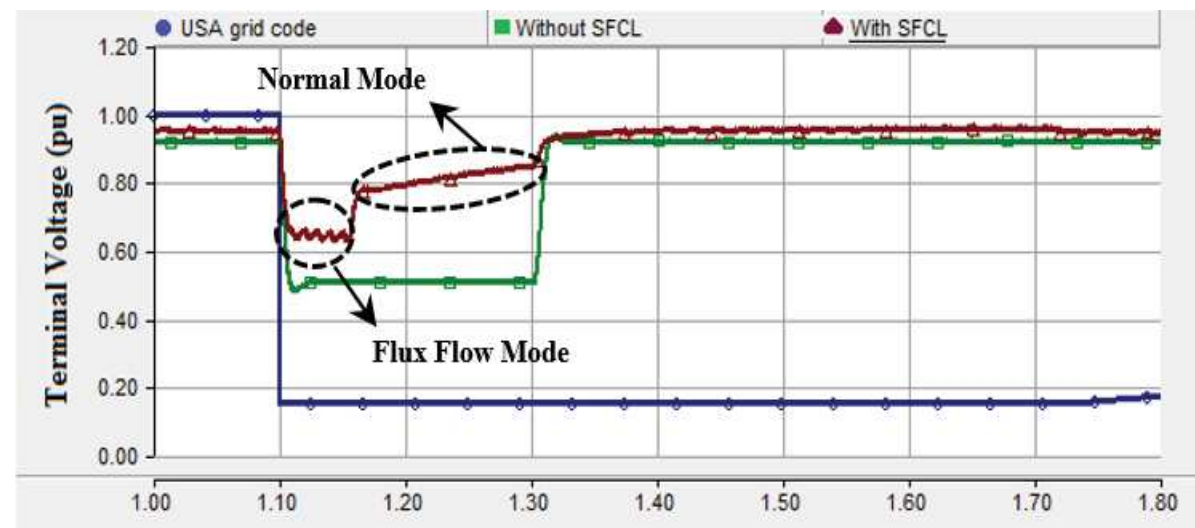

Time [sec]

Figure 3.7: Voltage-dip characteristic at the generator terminal.

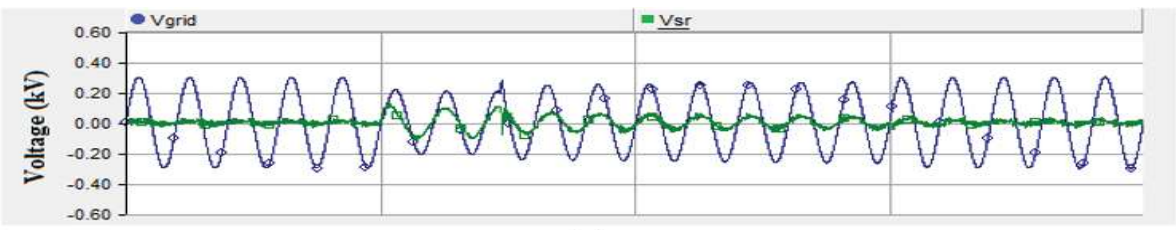

(a)

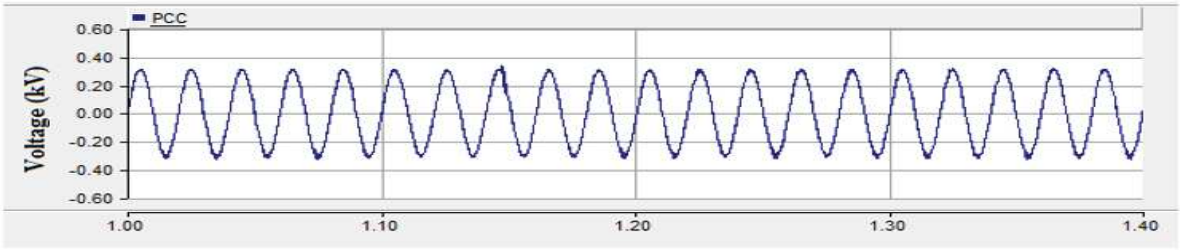

(b)

Time [sec]

Figure 3.8: Voltage sags compensation with SFCL. (a) Grid voltage and series APF voltage. (b) PCC voltage with compensation.

power $2 \mathrm{MW}$, with pitch angle control, and the reactive power absorbed from the grid is kept at zero with capacitor bank. The active power drops to approximately $0.8 \mathrm{MW}$ after fault occurred, without connecting SFCL. After connecting SFCL, the drop in the active power decreased, where it attained about 1.25 MW.

\subsection{LVRT Improvement of PMSG WTs Using a Resistive SFCL}

This section proposes an effective approach using resistive SFCL as the additional support along with conventional converter control strategy based on PI regulators to further 

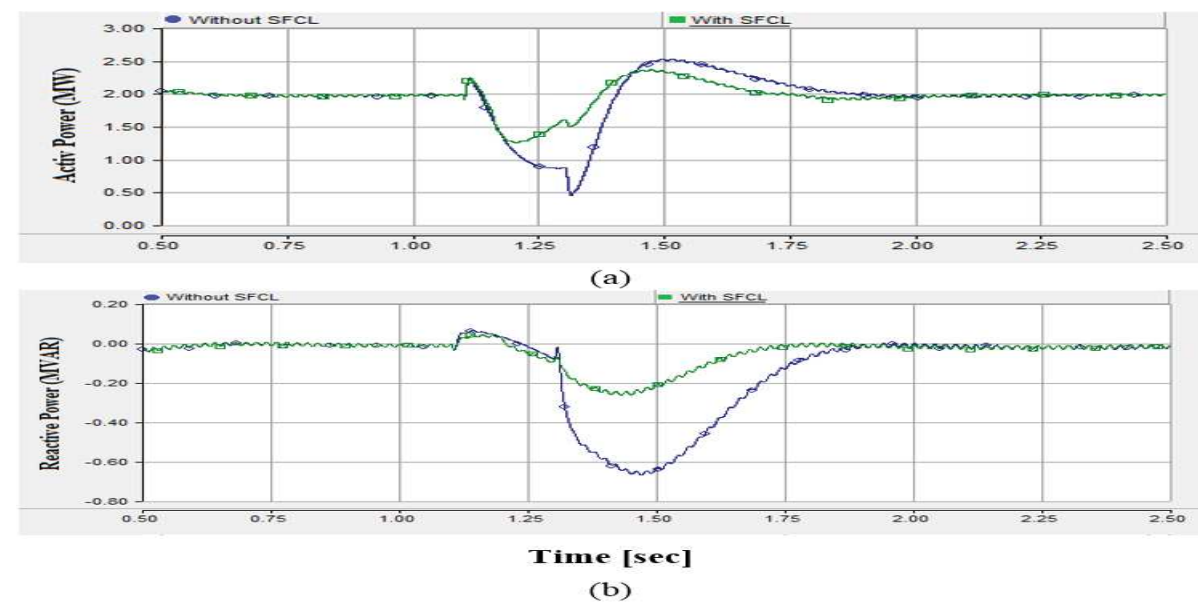

Figure 3.9: Active and reactive power delivered to the grid at PCC.

increase the rated active power of the installation, thereby enhancing dc-link voltage smoothness as well as the LVRT capability of the 50MW WPP. By using the RSFCL, the fault current is suppressed effectively and the voltage dip level of the WPP terminals is diminished, leading to enlarge the voltage safety margin of LVRT curve. The first-cycle suppression of a fault current by a RSFCL can also reduce the activation of pitch angle control and can decrease the effect of the extreme loads on the turbine components. These significant features of RSFCL can demonstrate that the proposed technique surpasses aforementioned methods using BCs and complicated nonlinear control system. However, a large WPP has a complicated structure using several components, and the inclusion of RSFCL composes this scheme more problematic for optimal performance of the system. Hence, the further effort in this study is centralized on the most-widely decision-making technique based on the analytic hierarchy process (AHP) [157], for the optimal performance of the combinatorial RSFCL and $50 \mathrm{MW}$ WPP. The technique creates the Pareto optimality for simultaneously optimizing 3-D alignment that rarely reported the power system literatures. Effectiveness of the proposed approach, using the Pareto optimality concept is verified by the numerical simulations. The optimization technique figures out all the nondominated solutions on the Pareto front at the end of the optimization run. 


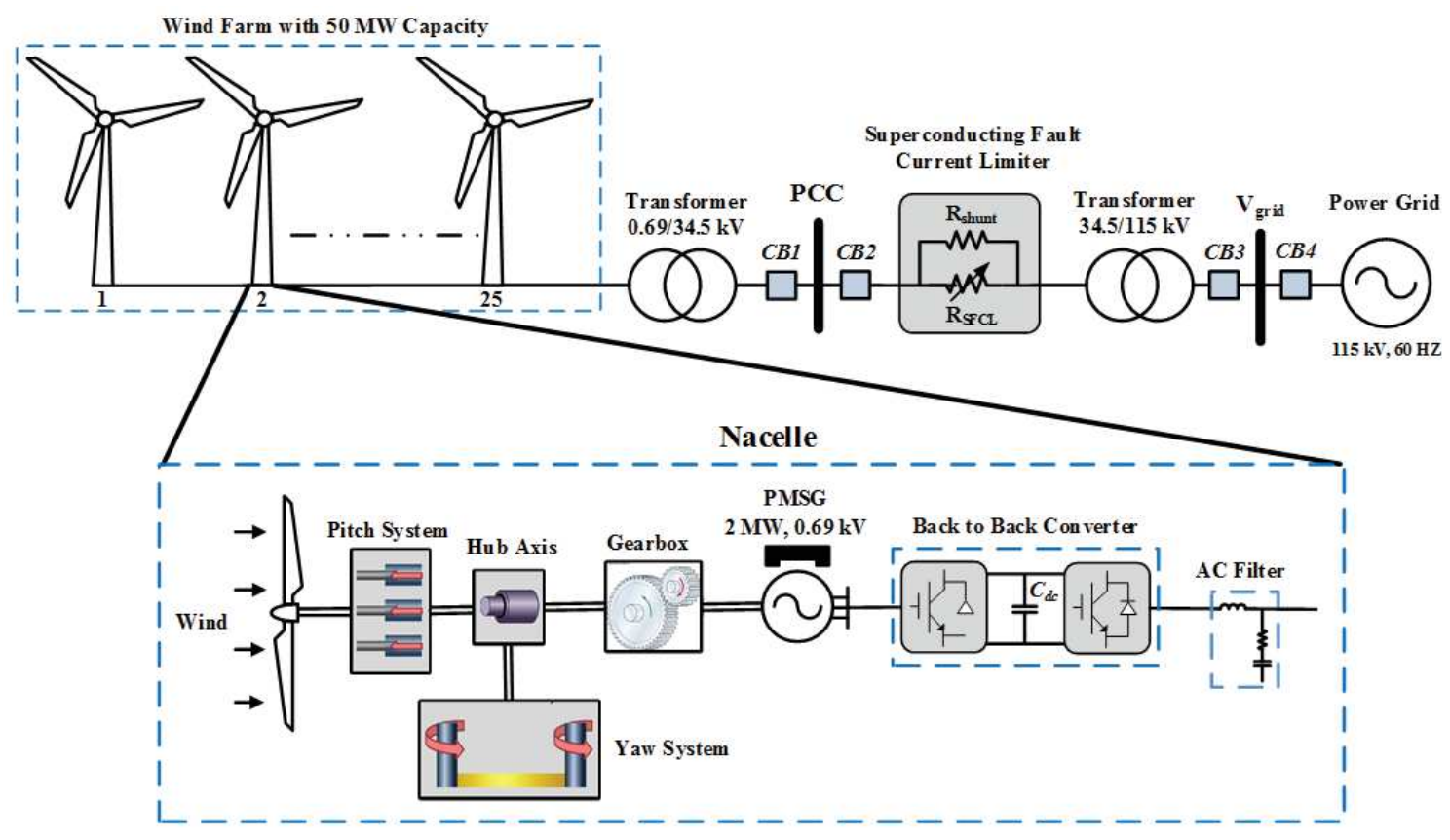

Figure 3.10: Proposed combinatorial PMSG-based WPP and RSFCL.

\subsubsection{Modeling of the PMSG-Based Wind Turbine}

The structure of the proposed system including a 50-MW PMSG WPP and resistive SFCL is schematically shown in Figure 3.10. The constituents of the wind turbine are aerodynamic, mechanical, and electrical parts. The generator is completely decoupled from the grid by power electronic converters (grid-side VSC and generator-side VSC which are connected back-to-back through the common dc-link capacitance). PMSG-based WTs may be represented as a combination of subsystems. The framework shown in Figure 3.11 is typically used for modeling purposes, in which the relevant mathematical model has been cited in the several literatures $[10,11,36]$, and it is summarily considered here.

\section{Aerodynamic Model}

According to the Betz theory, the aerodynamic power generated by the rotor is given by $[10]$

$$
P_{A}=0.5 \rho \pi R^{2} v_{\text {wind }}^{3} C_{p}(\lambda, \beta)
$$




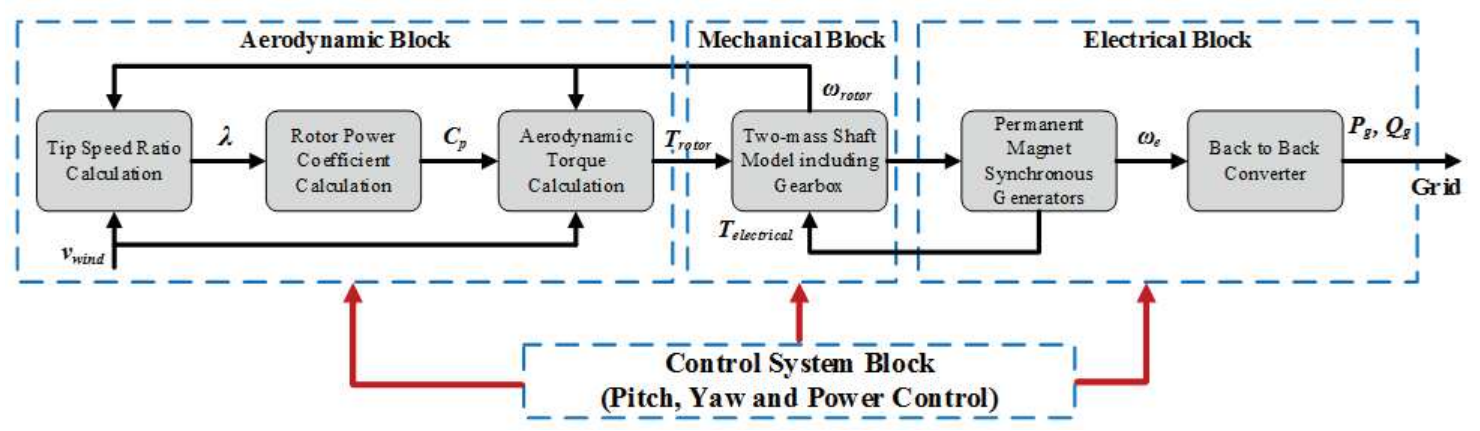

Figure 3.11: Subsystems model used in PMSG-based wind turbine.

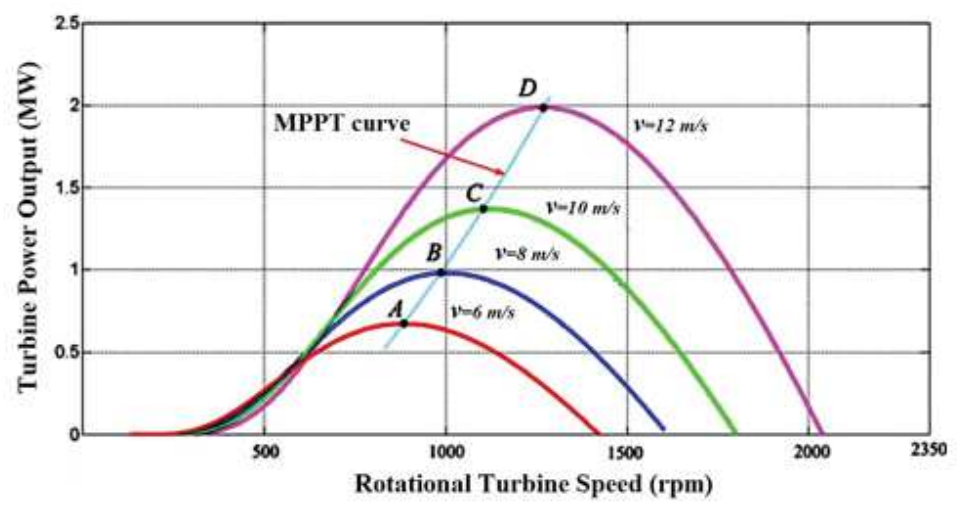

Figure 3.12: Wind turbine characteristics.

where $\rho$ is the air density $\left(\mathrm{kg} / \mathrm{m}^{3}\right), R$ is the radius of the blade $(\mathrm{m}), v_{\text {wind }}$ is the freestream wind speed $(\mathrm{m} / \mathrm{s})$, and $C_{p}(\lambda, \beta)$ is the rotor power coefficient. In the PMSG-based WT, the obtained power depends on $C_{p}$, which is a function of both tip speed ratio (TSR) $\lambda$ and blade pitch angle $\beta$, in which the TSR is defined as

$$
\lambda=\frac{R \omega_{\text {rotor }}}{v_{\text {wind }}}
$$

where $\omega_{\text {rotor }}$ is the rotational turbine speed. The numerical approximation of the power coefficient is given by following non-linear equations [43]

$$
\begin{gathered}
C_{p}(\lambda, \beta)=0.73\left(\frac{151}{\lambda_{t}}-0.58 \beta^{2.14}-13.2\right) e^{\frac{-18.4}{\lambda_{t}}} \\
\lambda_{t}=\frac{1}{\frac{1}{(\lambda-0.02 \beta)}-\frac{0.003}{\left(\beta^{3}+1\right)}}
\end{gathered}
$$




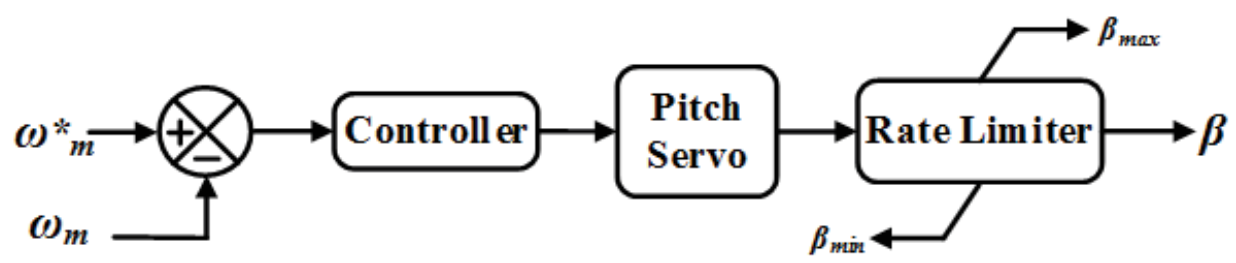

Figure 3.13: Conventional pitch angle control used in FSIG-based wind turbine.

The aerodynamic torque on the rotor $T_{\text {rotor }}$, which is produced by the blades of wind turbine can be calculated as $P_{A} / \omega_{\text {rotor }}$. Also, Figure 3.12 illustrates the relation between the rotational turbine speed and the aerodynamic power of the wind turbine $P_{A}$ for various wind speeds $v_{\text {wind }}$ with the blade pitch angle $\beta=0^{0}$. For each wind speed, the maximum power point can be acquired corresponding to determine the rotational speed that is expressed as [43]

$$
P_{\text {max }}=0.5 \rho \pi R^{2}\left(\frac{R \omega_{\text {rotor }}}{\lambda_{\text {opt }}}\right)^{3} C_{p}^{o p t}
$$

where $\lambda^{o p t}$ and $C_{p}^{o p t}$ are the optimum values of the tip speed ratio and power coefficient, respectively. For an average wind speed of $12 \mathrm{~m} / \mathrm{s}$, which is used in this section, the maximum turbine power output $2 \mathrm{MW}$ and rotational speed $1200 \mathrm{rpm}$ are obtained.

The aerodynamic of wind turbines is controlled with pitch control approaches, which have been developed for large WTs. The blades start to move around cut-in speed 4 $\mathrm{m} / \mathrm{s}$, while the optimal aerodynamic efficiency is achieved at the wind speed rated about $12 \mathrm{~m} / \mathrm{s}$. The extra power obtained from the wind speed between 4 and $12 \mathrm{~m} / \mathrm{s}$ may be smoothly curtailed by spinning the blades using a pitch control to avoid overloading the wind turbine system. Figure 3.13 depicts the conventional pitch angle regulator in which the input and output of the model are the rotational turbine speed $\omega_{\text {rotor }}$ and blade angle $\beta$, respectively.

The yaw system of a typical turbine is significantly slower than the pitch system and the structural dynamics. Since the yaw rates are so slow, that there is very little interac- 
tion with the rest of the system behavior, it is often not considered at all. If yawing is to be considered it can be modeled similar to the pitch system but with significantly lower bandwidth and rate limits. The gearbox also plays an essential role in the WTs to adapt low-speed, high-torque rotation of the turbine rotor into the faster rotation of the electrical generator. The critical issue in implementing the gearbox technology is the extreme loads, which may lead to misalignment of the drive train and a gradual failure of the gear components, consequently increasing the capital and operating cost of the WTs.

\section{PMSG Model}

Based on the reference frame theory $[6,11]$, stator voltage equations in a $d-q$ synchronous frame are modeled:

$$
\begin{gathered}
v_{s d}=R_{s} i_{s d}+L_{s} \frac{d i_{s d}}{d t}-\omega_{e} L_{d} i_{s q} \\
v_{s q}=R_{s} i_{s q}+L_{s} \frac{d i_{s q}}{d t}+\omega_{e} L_{d} i_{s d}+\omega_{e} \psi_{r}
\end{gathered}
$$

where $v_{s d}$ and $v_{s q}$ are the $d-$ and $q$-axis stator voltages, $i_{s d}$ and $i_{s q}$ are the $d-$ and $q$-axis stator currents, $R_{s}$ and $L_{s}$ are the stator resistance and inductance, $L_{d}$ and $L_{q}$ are the $d-$ and $q$-axis inductance, $\psi_{r}$ is the rotor flux, and $\omega_{e}$ is the electrical angular speed.

For the generator with surface-mounted permanent magnets, $d-$ and $q$-axis inductances are the equal $\left(L_{d}=L_{q}\right)$, resulting a simple interpretation of the electromagnetic torque $T_{\text {electrical }}$ and aerodynamic torque on the rotor $T_{\text {rotor }}$ expressed as

$$
\begin{gathered}
T_{\text {electrical }}=\frac{3}{2} p \psi_{r} i_{\text {sq }} \\
T_{\text {rotor }}-T_{\text {electrical }}=J \frac{d \omega_{\text {rotor }}}{d t}+b \omega_{\text {rotor }}
\end{gathered}
$$

where $p$ is the number of machine pole pairs, $J$ is moment of inertia for turbine-generator, $\omega_{m}$ is shaft mechanical speed, and $b$ is friction coefficient. 


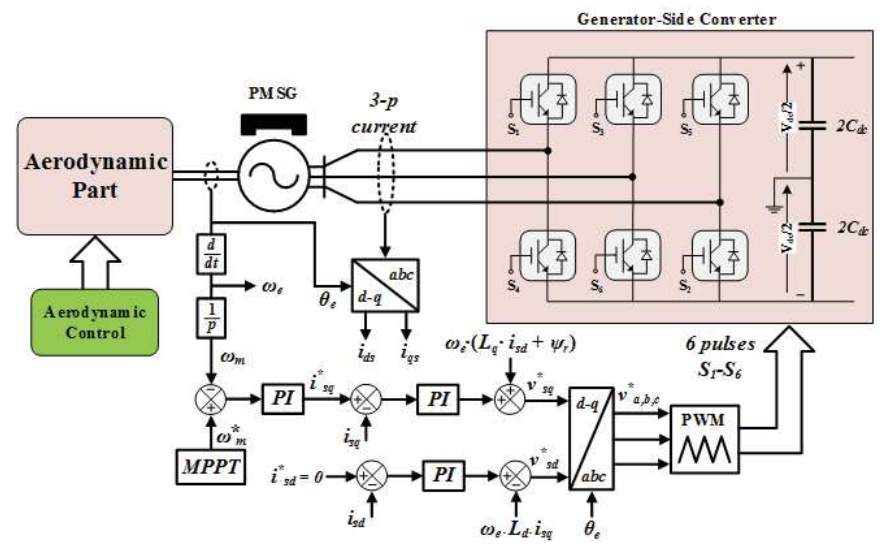

(a)

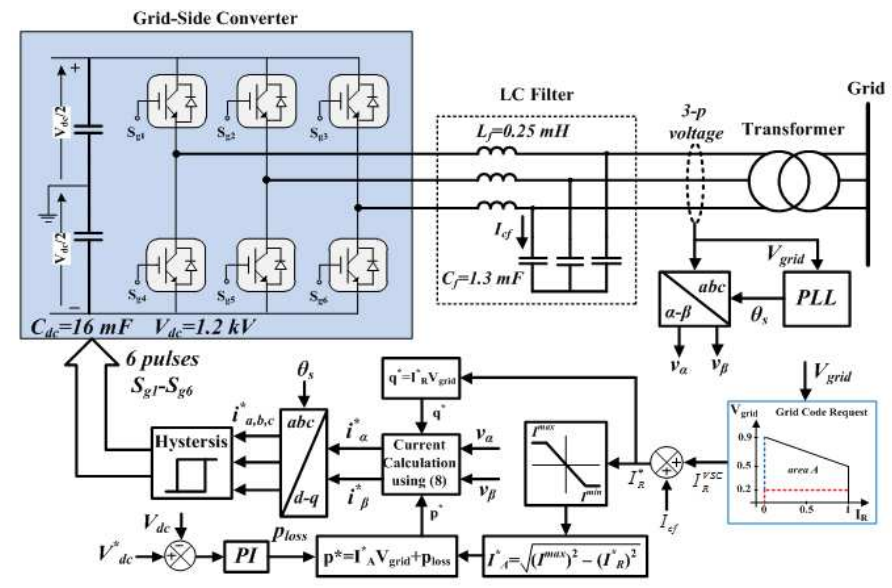

(b)

Figure 3.14: Proposed power control strategy for WPP. (a) Control block diagram of the generator-side VSC. (b) Control block diagram of the grid-side VSC.

\subsubsection{Power Control Strategy}

Details of the proposed power control scheme for PMSG based on full-power converter topology is illustrated in Figure 3.14. As it can be seen, it is schematically divided in two main blocks. On the one hand, controlling the active and reactive power of PMSG is obtained by generator-side VSC. Also, the management of the active and reactive power released to the grid by the PMSG along with the dc-link regulation is accomplished via the grid-side VSC. The active and reactive power references to be injected by the gridside VSC are obtained, so that the whole wind farm can fulfill the grid code requirements. 


\section{Generator-Side VSC Control}

The control block diagram of generator-side VSC is shown in Figure 3.14(a), which is based on stator voltage equations (3.11) and (3.12) obtained in $d-q$ synchronous frame. Several maximum power point tracking (MPPT) algorithms have been reported for PMSG-based WT. The outcome of MPPT provides the reference value of the rotational turbine speed $\left(\omega_{\text {rotor }}^{*}\right)$ for the generator-side VSC controller. This section mainly focuses on the converter control, and hence, the MPPT control method was not discussed. The speed reference $\omega_{m}^{*}$ is acquired by the maximum power point tracking (MPPT) method mentioned in (3.10) in order to extract the maximum amount of power with the actual wind force, while the rotational speed error is given as the input to a PI controller in order to generate the $q$-axis stator current command $\left(i_{s q}^{*}\right)$. Also, the reactive power produced by the wind turbine is regulated at zero $\left(i_{s d}^{*}=0\right)$ for unity power factor operation. The error between the reference $d q$-axis currents and the actual $d q$-axis currents, $i_{s d}, i_{s q}$ are used as inputs to the linear PI controllers to produce $d q$-axis voltage commands, $v_{s d}^{*}, v_{s q}^{*}$ after the decoupling. The angle $\theta_{e}$ calculated from the rotational speed of the PMSG is applied in park transformation to engender gate signals using the carrier wave of pulse width modulation (PWM) operation.

\section{Grid-Side VSC Control}

The proposed grid-side converter controller is provided in Figure 3.14(b) to calculate the current references to be inserted by the grid-side VSC in order to fulfill the grid code requirements. Further, this controller preserves the dc-link capacitor voltage at the set value $1.2 \mathrm{kV}$, which assures the active power swapping from PMSG to the grid. In the steady state condition, the maximum capacity of the current, $I_{\max }$ is used to produce 2 MW active power $\left(I_{R}^{V S C}=0\right)$. In the next stage, the both of the $p^{*}$, which is added to the PI controller from the dc-link voltage regulator, and $q^{*}$, are transformed into the 
instantaneous power $\alpha-\beta$ method based on $\alpha-\beta-0$ reference frame [30]. It has been mathematically formulated as

$$
\left[\begin{array}{c}
i_{\alpha}^{*} \\
i_{\beta}^{*}
\end{array}\right]=\frac{1}{v_{\alpha}^{2}+v_{\beta}^{2}}\left[\begin{array}{cc}
v_{\alpha} & -v_{\beta} \\
v_{\beta} & v_{\alpha}
\end{array}\right]\left[\begin{array}{c}
\tilde{p}-\bar{p}_{\text {loss }} \\
q
\end{array}\right]
$$

The angle $\theta_{s}$ for the Park transformation is detected from the three-phase voltages at the low-voltage side of the grid-side transformer by using a phase-locked loop (PLL). Finally, gate signals are generated for grid side VSC switches using the Hysteresis module [158] shown in Figure 3.14(b). Under a grid voltage dip, the reactive current, $I_{R}^{V S C}$ in proportion to the voltage reduction should be provided during the fault in order to meet the LVRT requirement according to the characteristic shown in Figure 1.1(b). As can be seen in Figure 3.1(b), the injection of the reactive power has the highest priority in area A, but free capacity of current, $I_{A}^{*}$ must also be utilized to retain the active power production related to the voltage sag magnitude, while the generator continues to provide active power at nominal value. In this case, dc-link voltage exceeds its safety limits, leading a system malfunction or even component failure. However, the rapidly rising dclink voltage under system fault is difficult to be avoided by only using the PI controller.

For this reason, this section proposes a RSFCL installed in outside of the wind farm, as shown in Figure 3.10. RSFCL makes it possible to suppress the dc-link voltage fluctuations by limiting the magnitude of the fault current, thereby increasing the output active power capacity and improving the LVRT capability of the wind farm. A further analysis is accomplished in Section 3.3.4.

\subsubsection{Electro-Thermal Modeling of a RSFCL}

The resistive superconducting fault current limiters (RSFCLs) have been launched and introduced into the network as a self-healing technology to curb prospective fault currents immediately to a manageable level by suddenly raising the resistance value [30,122]. Fur- 


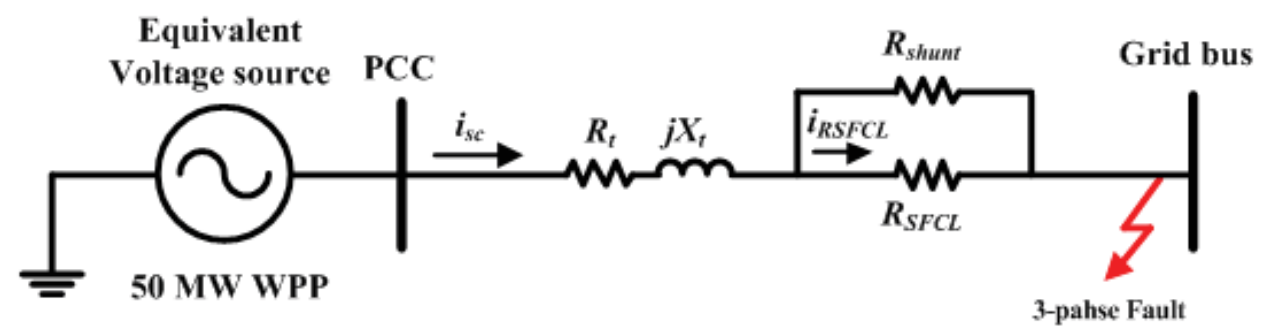

Figure 3.15: Equivalent circuit of proposed system in fault condition.

thermore, after the fault current is profitably repressed, the RSFCL can be restored to the primary state without additional aid. Considering the superconducting material, BSCC2223, the conductor has commonly been utilized for most of the tentative RSFCLs. RSFCL has a simple structure with a lengthy superconductor wire inserted in series with the transmission lines. With the recent breakthrough of second-generation high-temperature superconductor (HTS) wires, the SFCL has become more viable. To preserve the superconductor from detrimental hot spots during the operation, the shunt resistance, $R_{\text {shunt }}$ is essential. This parallel resistance must be contacted all over the length of the superconductor, and it regulates the controlled current to elude over-voltages likely occurring when the resistance of the superconductor increases much quicker. The current limiting behavior of the RSFCL are presented in Equation (3.5).

According to the equivalent circuit of the proposed combination system, shown in Figure 3.15, if the asymmetrical component of the fault current is ignored, the shortcircuit current through the RSFCL branch can be stated by the following equations

$$
i_{R S F C L}(t)=\frac{R_{\text {shunt }}}{R_{S F C L}+R_{\text {shunt }}} \times \frac{V_{m}}{\sqrt{R^{2}+\left(L_{T} \omega\right)^{2}}} \sin (\omega t)
$$

where $R=R_{\text {trans }}+R_{\text {shunt }} \| R_{S F C L}, L_{T}$ is the inductance of the transformer, and $V_{m}$ is the magnitude low voltage side of interfacing transformer. The total fault energy dissipated in the HTS tapes for three phases, $Q_{s c}$ is calculated using (3.17), where $\Delta t_{s c}$ is the duration 
of the fault [159].

$$
Q_{s c}=3 \int_{\Delta t_{s c}} R_{S F C L} i_{R S F C L}^{2}(t) d t
$$

Substituting (3.16) into (3.17) gives the following

$$
Q_{s c}=\frac{3 V_{m}^{2} R_{S F C L}}{R^{2}+\left(L_{T} \omega\right)^{2}}\left[\frac{\Delta t_{s c}}{2}-\frac{\sin \left(2 \omega \Delta t_{s c}\right)}{4 \omega}\right]
$$

The RSFCL model should be a reasonable approximation of transient SFCL behavior during faults and, therefore, should consider thermal properties. The thermal model of RSFCL has been generically estimated as follows [21]

$$
T(t)=T_{0}+\frac{1}{C_{s c}} \int\left[Q_{s c}(t)-P_{\text {cool }}(t)\right] d t
$$

where $T_{0}$ is ambient temperature, $C_{s c}$ is the heat capacity of the superconductor, and $P_{\text {cool }}$ is the power cooling.

\subsubsection{Numerical Simulation Analysis}

The wind farm shown in Figure 3.10 consists of the 25 wind turbines rated at $2 \mathrm{MW}$, which totally supply the maximum $50 \mathrm{MW}$ to the grid, where the base wind speed is designed as $12 \mathrm{~m} / \mathrm{s}$ based on $\left(p-\omega_{\text {rotor }}\right)$ characteristic curve (Figure 3.12). To perfor$\mathrm{m}$ a realistic design, all aspects of a WT need to be considered. Thus, a holistic wind turbine model was utilized including detailed aerodynamics and structural, mechanical, and electrical systems using MATLAB/SIMULINK file [160]. Indeed, this model covers a three-dimensional mechanical model of the tower, nacelle, and blades modeled in

SimMechanics, hydraulic pitch actuators, electrical yaw actuators, a full generator and electrical grid model. The modeling of the RSFCL was also accomplished using the MATLAB programming to combine its electrical and thermal properties as discussed in Section 3.3.3. A top-level view of the model is shown in Figure 3.17. The characteristics of the preferred wind farm and selected resistive RSFCL parameters are given in Table 3.3 and Table 3.4 . 

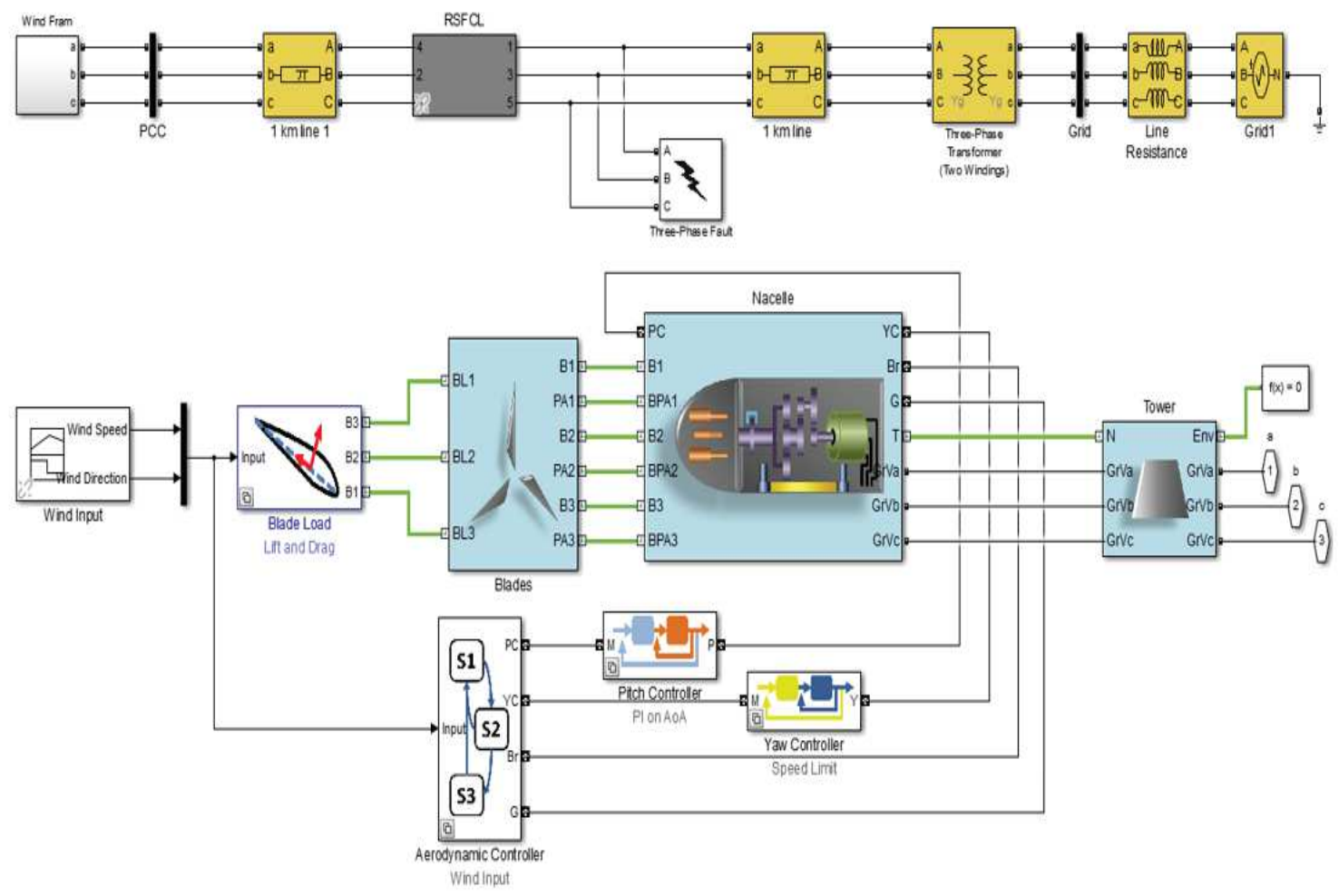

Figure 3.16: Combinatorial PMSG-based WT and RSFCL model using SimPower Systems.

\section{Effect of RSFCL on DC-Link Voltage Smoothness}

For simplification proposes, in this section we only concentrate on details of turbine's electrical model in order to analyze the impact of RSFCL on dc-link voltage smoothness.

Suppose a symmetrical three-phase fault considered at the integration point with the grid, is appearing at $4 \mathrm{~s}$ and cleared after $\Delta t_{s c}=0.2 \mathrm{~s}$. To assess the damping behavior of the RSFCL, simulations are carried out for without and with the presence of the RSFCL. The expediency of the RSFCL component for managing the fault current as well as resistance and temperature variations of SFCL is demonstrated in Figure 3.18. The peak current for phase a in the pre-fault value is $850 \mathrm{~A}$ and then exceeds $14.2 \mathrm{kA}$ without connecting RSFCL whereas with SFCL incorporated on the main road of the wind farm, the fault current is limited effectively to reach about $5.1 \mathrm{kA}$ (see Figure 3.18(a)). Figure 3.18(b) illustrates the limiting resistance of the SFCL, which went up to $7.1 \Omega$ in the flux 
Table 3.3: Characteristics of the wind farm induction generator.

\begin{tabular}{|l||l|l|}
\hline Symbol & Quantity & Value \\
\hline$P_{\text {rated }}$ & Rated generator power & $2 \mathrm{MW}$ \\
$v_{\text {wind }}$ & Rated wind speed & $12 \mathrm{~m} / \mathrm{s}$ \\
$R$ & Blade radius & $46 \mathrm{~m}$ \\
$\rho$ & Air density & 1.225 \\
$C_{p}^{\text {opt }}$ & Optimal power coefficient & 0.45 \\
$\lambda^{o p t}$ & Optimal tip speed ratio & 8.32 \\
$V_{r m s}$ & Rated rms line-line voltage & $0.69 \mathrm{kV}$ \\
$\psi_{r}$ & Rated rotor flux & $17 \mathrm{~Wb}$ \\
$\omega_{m}$ & Rated speed & $1200 \mathrm{rpm}$ \\
$R_{s}$ & Stator winding resistance & $0.017 \mathrm{pu}$ \\
$L_{s}$ & Stator winding inductance & $0.064 \mathrm{pu}$ \\
$R_{r}$ & Rotor winding resistance & $0.103 \mathrm{pu}$ \\
$L_{d}, L_{q}$ & d, -axis synchronous inductance & $8.75 \mathrm{mH}$ \\
$P$ & Poles pairs & 3 \\
$H$ & Mechanical time constant & $2.5 \mathrm{sec}$ \\
\hline \hline
\end{tabular}

Table 3.4: Parameters of SFCL.

\begin{tabular}{|l||l|l|}
\hline Symbol & Quantity & Value \\
\hline$T_{c}$ & Critical temperature for HTS & $99^{0} \mathrm{~K}$ \\
$T_{0}$ & Temperature of cooling system & $77^{0} \mathrm{~K}$ \\
$I_{c 0}$ & Critical current & $5 \mathrm{kA}$ \\
$C_{p}$ & Specific heat of HTS & $3 \mathrm{MJ} m^{-3} K^{-1}$ \\
$P_{c o o l}$ & Power of cooling & $700 \mathrm{~kW}$ \\
$V_{s c}$ & HTS Volume & $3 \mathrm{e}-4 m^{3}$ \\
$A_{s c}$ & HTS Cross section & $1 \mathrm{e}-6 m^{2}$ \\
$R_{s h}$ & Shunt resistance of HTS & $120 \Omega$ \\
$\rho_{n}$ & Normal resistivity & $4 \mathrm{e}-8 \Omega m$ \\
$\rho_{f}$ & Flux flow resistivity & $1 \mathrm{e}-9 \Omega m$ \\
\hline \hline
\end{tabular}

flow state and rise to reach a normal stat value of $15 \Omega$ after ten cycles of the fault. A retrieval of the Figure 3.18(b) and 3.18(c) will determine, when a fault takes place at $t$ $=4 \mathrm{~s}$, the quench time ( $\mathrm{a}$ transition from a superconducting mode to a resistive mode) is initiated by going through the flux-flow state during of $0.1 \mathrm{~s}$ and then to the normal state at a temperature rise of $90^{\circ} \mathrm{K}$ (critical temperature for HTS tap). 


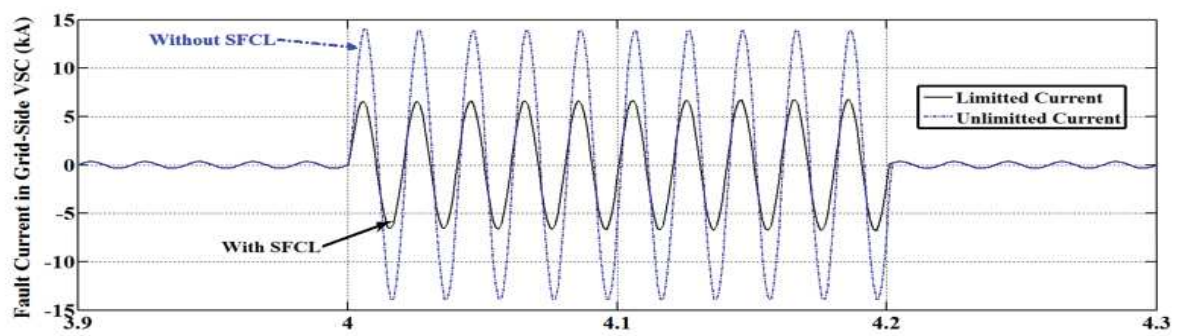

(a)

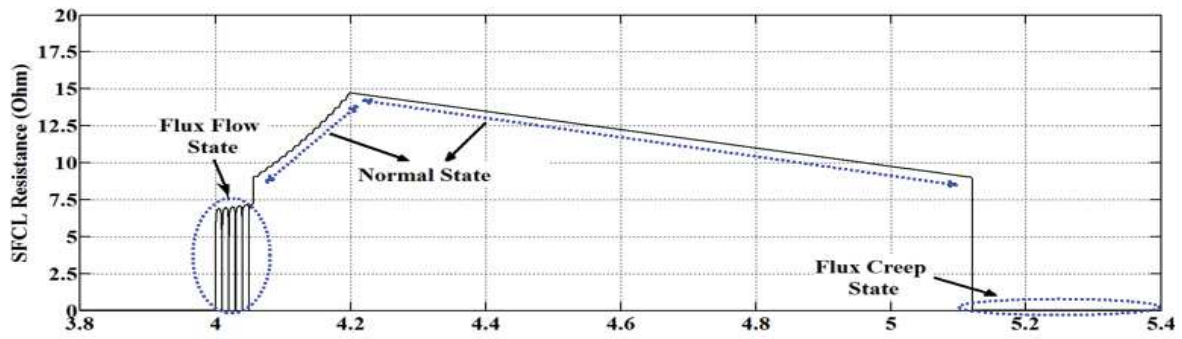

(b)

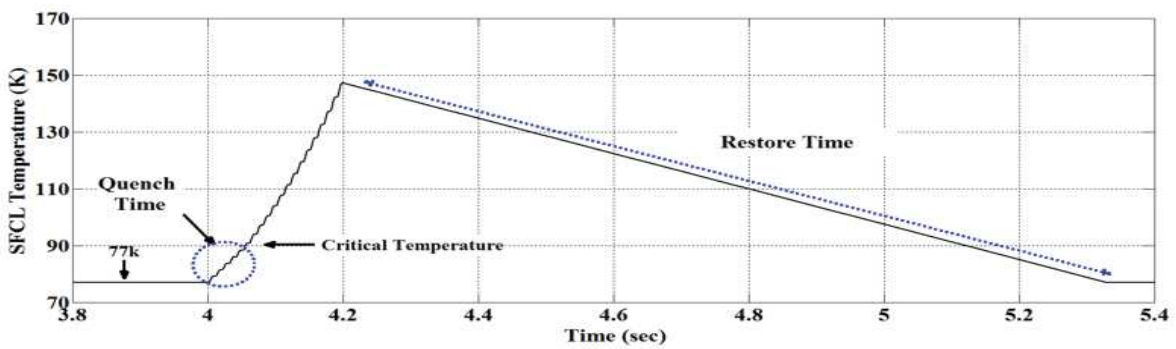

(c)

Figure 3.17: RSFCL model response. (a) Fault current waveform without and with RSFCL in a single-phase system. (b) Resistance variation in flux flow and normal state. (c) Temperature rise.

Figure 3.19(a) shows the voltage profile on PCC in the proposed integration system during a three-phase short circuit. In the absence of the reactive injection and RSFCL, the voltage reduction of $70 \%$ occurs. In this case, the voltage at the PCC cannot be restored to the nominal value because of an instability issue on the proposed system and WPP must be disconnected from the grid. With the adoption of the reactive injection control, the voltage dip is decreased, reaching $50 \%$ before recovering immediately to the nominal value upon clearing the fault. Based on the reactive power support requirement (Figure $1.1(b))$, for a $50 \%$ voltage reduction, all the capacity of the wind farm is occupied by reactive power. 


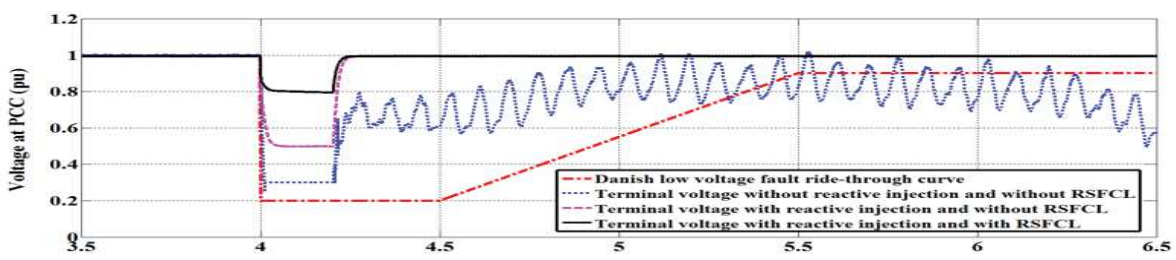

(a)

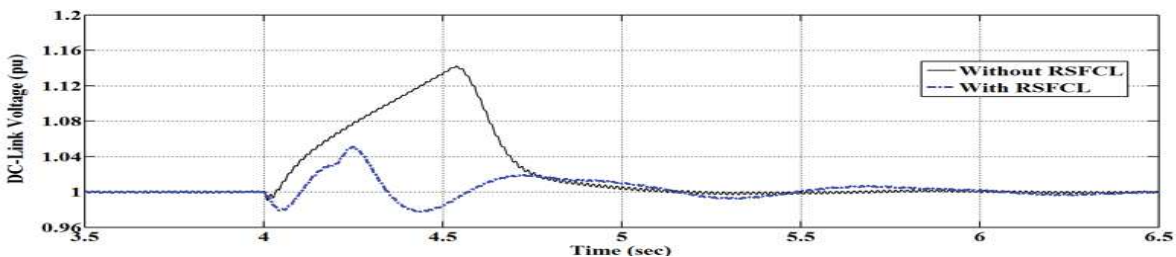

(b)

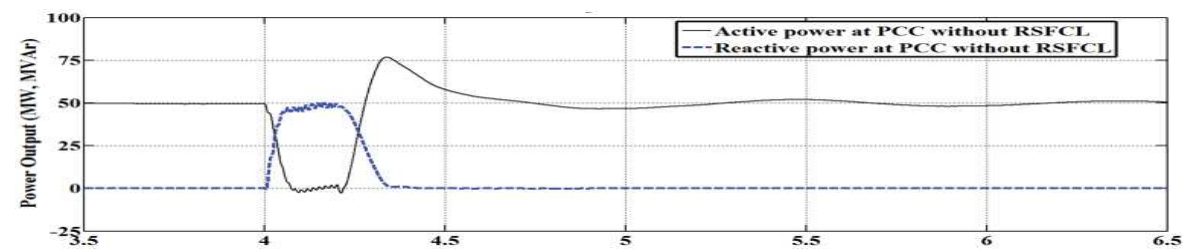

(c)

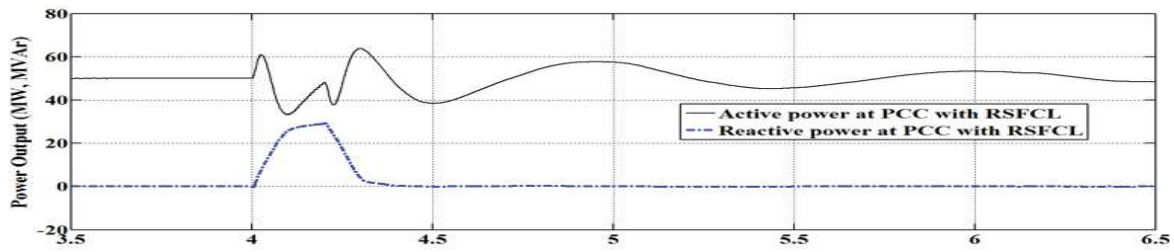

(d)

Figure 3.18: Operation of the proposed combinatorial WPP and RSFCL during and after fault (a) Voltage profile at wind farm terminal (b) dc-link voltage with and without RSFCL. (c) Active and reactive power at the PCC without the RSFCL. (d) Active and reactive power at the PCC with the RSFCL.

As can be observed in Figure 3.19(c), the reactive power injected during the fault allows the wind farm to satisfy the specifications of grid code requirements such as increasing the LVRT capacity. However, due to the lack of output active power in the grid-side VSC and consequently earlier-mentioned unbalanced power during the fault, the dc-link voltage is significantly increased to about $1.14 \mathrm{pu}$, where a regular reactive power control with no RSFCL is used i.e., 14.58\% over voltage (Figure 3.19(b)). This effort proposes the RSFCL as an additional supporting method besides the reactive power control to improve the LVRT capability and dc-link voltage smoothness of the wind farm. This method 


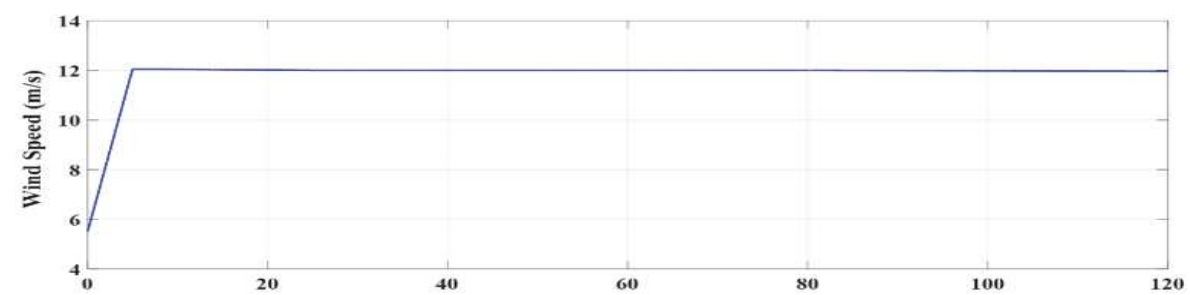

(a)

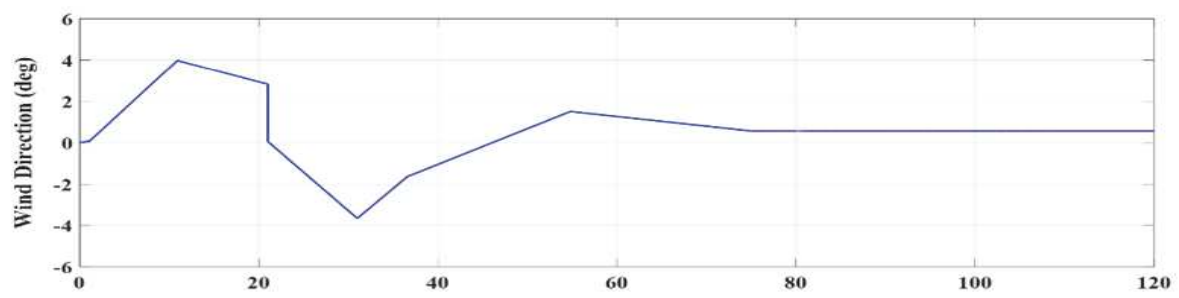

(b)

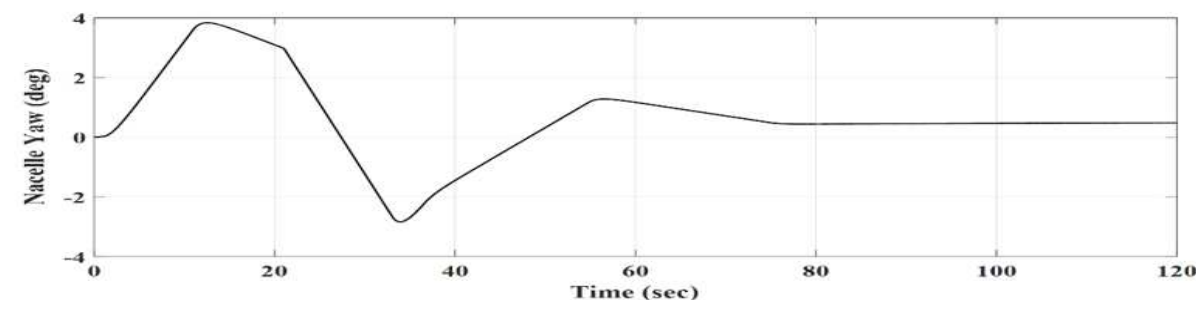

(c)

Figure 3.19: Operational setting for proposed system. (a) Wind speed. (b) Wind Direction. (c) Nacelle yaw control performance.

increases the voltage stability margin with respect to the LVRT curve, in which voltage sag is dramatically improved to $20 \%$. Figure 3.19(d) illustrates the active/reactive power output of the wind farm with the RSFCL, in which they are considerably kept at rated value $50 \mathrm{MW}$ and 0 MVAr before the fault, respectively. After installing RSFCL, the drop in the active power decreased from $0 \mathrm{MW}$ to $32 \mathrm{MW}$ and back to the normal operation gradually as the fault is cleared. That is, the RSFCL presence gives rise to the retaining of the active power production for the PMSG-WPP by approximately $60 \%$ during the fault condition. The impact of the installed RSFCL (along with a regular reactive power control) on the dc-link voltage is effectively demonstrated by declining it to 1.05 p.u (only $5 \%$ over voltage). 


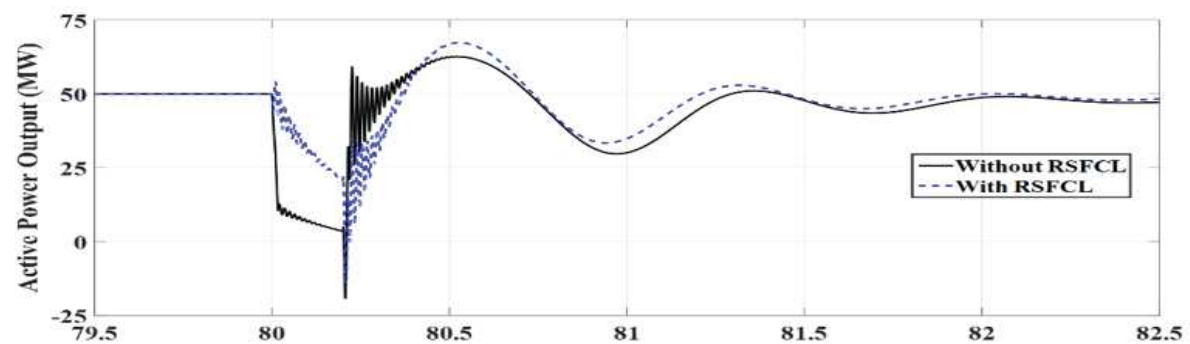

(a)

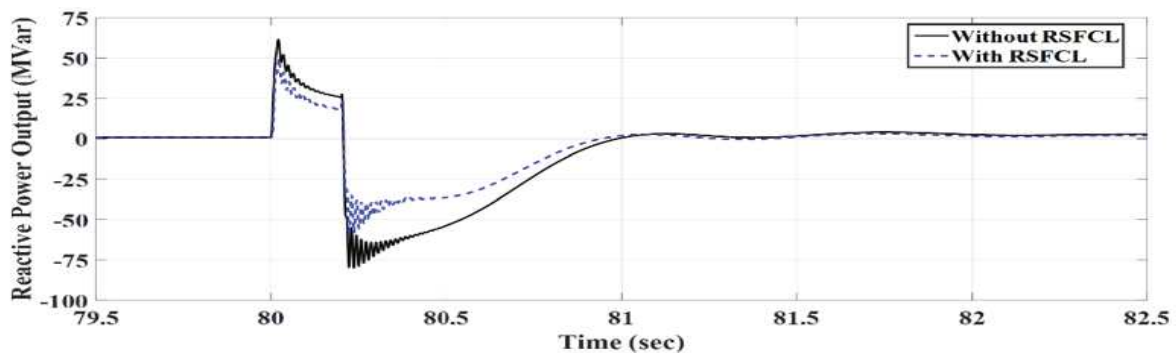

(b)

Figure 3.20: Power delivered to the grid at PCC with and without RSFCL. (a) Active power output. (b) Reactive power output.

\section{Effect of RSFCL on Extreme Load Reduction}

In this section, in order to analyze the impact of RSFCL on WT extreme loads, a holistic wind turbine model is utilized to simulate the detailed aerodynamics and mechanical aspects of a WT. It assumed that wind speed reaches to rated value of $12 \mathrm{~m} / \mathrm{s}$ at $\mathrm{t}=5 \mathrm{~s}$ after starting up the WT as shown in Figure 3.20(a). It also assumed that there is a fluctuant trend in wind direction (see Figure 3.20(b)) in order to demonstrate the effective performance of the yaw control system, in which the yaw system drives motor action to rotate the turbine nacelle to encounter the wind and reduce the difference between the wind direction and the turbine angle as show in Figure 3.20(c). It is notable that generator should be connected to the grid when the rotational speed reaches a rated value of $1200 \mathrm{rpm}$. For comparison purposes, the dynamic performance of the proposed system were carried out for without and with the presence of the RSFCL. Similarly, a three-phase fault is applied at $\mathrm{t}=80 \mathrm{~s}$ and is cleared after $200 \mathrm{~ms}$, resulting in $80 \%$ voltage dip at the PCC as shown in Figure 3.19(a). 


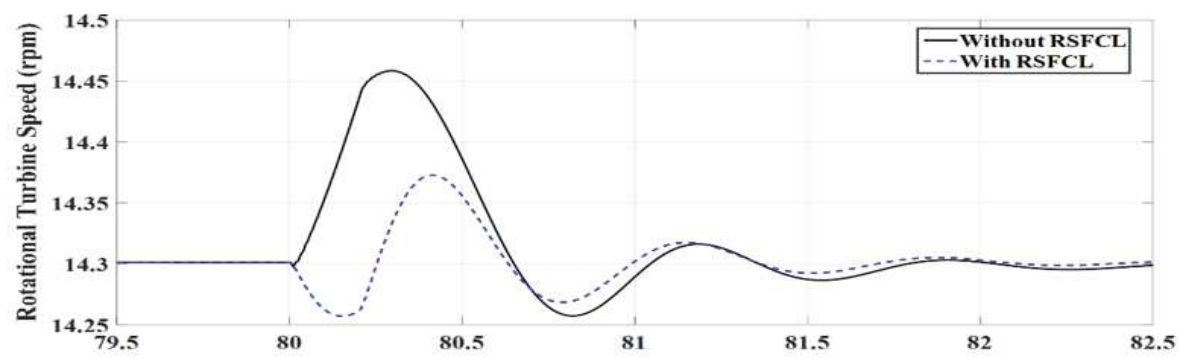

(a)

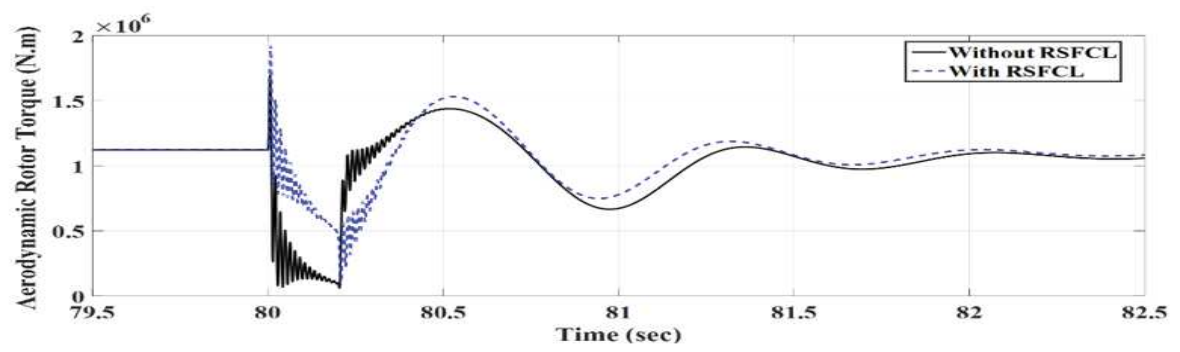

(b)

Figure 3.21: Dynamic performance of the turbine side with and without applying the RSFCL. (a) Rotational turbine speed. (b) Aerodynamic rotor torque.

Figure 3.21 show the active and reactive power output of the wind farm with and without the RSFCL, in which they are considerably kept at rated value $50 \mathrm{MW}$ and 0 MVAr before the fault, respectively. The active power drops to approximately $5 \mathrm{MW}$ after fault occurred, without connecting SFCL. After connecting SFCL, the drop in the active power decreased, where it is attained about $30 \mathrm{MW}$. Moreover, from these figures it is seen that the grid-side VSC can provide necessary reactive power for LVRT improvement during faults condition.

Rotational speed and aerodynamic torque responses of the rotor turbine and generator are shown in Figure 3.22 and Figure 3.23, respectively. As it can be seen, the rotational speeds and aerodynamic torque increase during the fault period which may lead to power system instability and is detrimental for the turbine generator system if the fault duration is long and proper auxiliary devices are not used (no controller). However, RSFCL can limit the rate of rising of machine speed and the aerodynamic torque imposed on rotor/shaft in order to make better stability. Finally, the impact of the installed RSFCL 


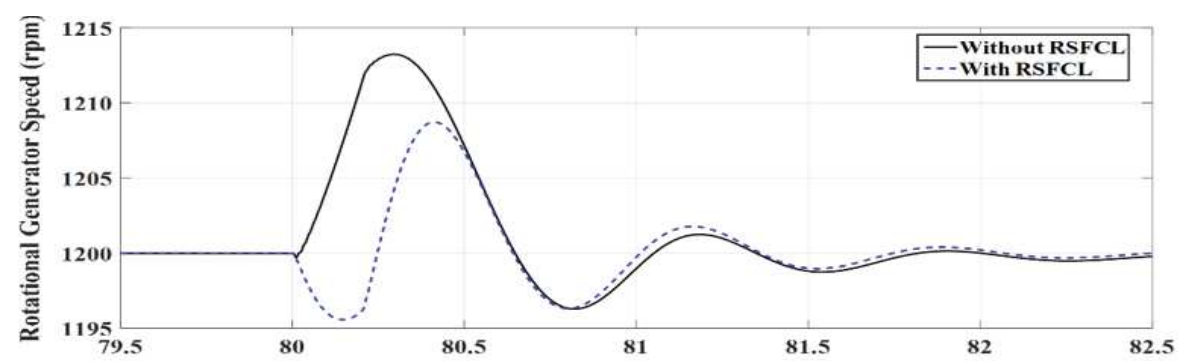

(a)

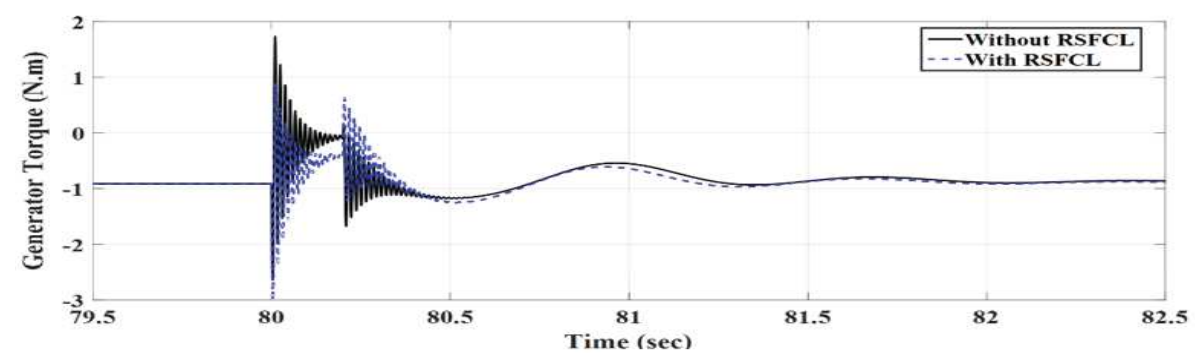

(b)

Figure 3.22: Dynamic performance of the generator side with and without applying the RSFCL. (a) Rotational generator speed. (b) Generator torque.

on pitch actuator force is effectively demonstrated by $50 \%$ reduction during the fault in proportion to the case with no using RSFCL. Therefore, RSFCL can be a promising solution for wind turbine controller performance with respect to extreme loads happening to mechanical part during the severe disturbances.

\subsubsection{Optimal Scheme Performance}

The obtained results in Section 3.3.4 for the proposed combinatorial 50-MW wind farm and RSFCL confirmed that further improvements in dc-link smoothness, extreme load, and LVRT capability of a wind farm can be achieved by increasing the SFCL resistance as much as possible. However, as stated in (3.18), the high-resistance SFCL means a substantial amount of energy is dissipated in the form of heat, resulting damage on SFCL construction and cooling system. This large energy dissipation would lengthen the recovery time of the RSFCL (transition from resistive state to superconducting state) after clearing the fault. Also, as stated in Section 3.3.2, for overcoming the unbalance power between the generator and converter, the active power output of the wind farm, $P_{W P P}$ 
should be appropriately increased during the fault to diminish the fluctuations of dc-link capacitor voltage. However, depending on the grid code, reactive power production has highest priority during the fault, occupying some portion of the maximum capacity of apparent power, and leading reduction in $P_{W P P}$. Hence, there is a tradeoff between three above mutually contradicting criteria, SFCL resistance, energy dissipation, and active power output of the WPP in order to achieve an optimal design of combinatorial 50-MW wind farm and resistive SFCL.

For optimization purposes, this section implements multi-criteria decision making (MCDM) methodology based on analytical hierarchy process (AHP) detailed in [30]. One of the outstanding characteristics of the MCDM technique is the creation of the Pareto optimality for simultaneous multiobjective optimization in which algorithm figures out all the nondominated solutions on the Pareto front (optimality) at the end of the optimization run. AHP is established as beneficial technique providing the promising solutions to the complicated decision-making problems with different criteria. The proposed optimization model contains three predefined criteria and two constraints that are expressed as

$$
\begin{gathered}
\operatorname{Min}\left\{\frac{1}{R_{S F C L}}, \frac{1}{P_{W P P}}, Q_{s c}\right\} \\
\text { Subject to }\left\{\begin{array}{l}
T_{S F C L}^{\max }-423<0, \\
\text { point }\left(V_{\text {grid }}, \Delta t_{s c}\right) \text { within Area } A .
\end{array}\right.
\end{gathered}
$$

where the maximum $R_{S F C L}$, the maximum $P_{W P P}$, and the minimum $Q_{s c}$ are desirable. The proposed system (combination of 50-MW WPP and RSFCL) should be designed in such a way that the following criteria are satisfied: 1) $T_{S F C L}^{\max }<432^{0}$ for safe solder melting; and 2) fulfill Danish grid code requirement including LVRT and reactive power support requirement. 
Table 3.5: Limits of Variables for Optimization Problem.

\begin{tabular}{|l|l|l|l|l|}
\hline Symbol & Quantity & Min Value & Start & Max Value \\
\hline$V_{s c}$ & Volume of HTS & $5 \mathrm{e}-4 m^{3}$ & $5 \mathrm{e}-4 m^{3}$ & $1 \mathrm{e}-3 m^{3}$ \\
$A_{s c}$ & Cross section of HTS & $1 \mathrm{e}-6 m^{2}$ & $5 \mathrm{e}-6 m^{2}$ & $1 \mathrm{e}-5 m^{2}$ \\
$\Delta t_{s c}$ & Duration of the fault & $0 \mathrm{~s}$ & $0.2 \mathrm{~s}$ & $0.8 \mathrm{~s}$ \\
\hline
\end{tabular}

Based on (3.5), any change in the dimensions of the superconducting wires as well as fault durations may affect fault current limiting performance of RSFCL, and consequently the optimum design of the proposed system. Therefore, in this optimization, variable parameters are superconducting wire volume $\left(V_{s c}\right)$, superconducting wire cross section $\left(A_{s c}\right)$ and duration of the fault $\left(\Delta t_{s c}\right)$. The constraints of the selected variables for the optimization problem are shown in Table 3.5.

Considerately, if each variable is changed in 10 steps, three variables would create $10^{3}=1000$ alternatives when utilized in the electrical simulation model. The cases that exceed the predefined optimization constraints must be omitted from feasible options, which are 378 cases. The goal of AHP method is to find a best case (desired solution) among the remnant number of 784 alternatives, which can maximize each criterion satisfaction. Basically, for 784 alternatives $\left(A_{i}, i=1,2,, 784\right)$ and 3 criteria $\left(C_{j}, j=1,2,3\right)$, there are four steps considering decision problems by AHP as follows:

Step 1) Scrutinize the relation between objectives, criteria and alternatives to build the multi-layers hierarchical structure. Figure 3.24 shows the multi-layers hierarchical structure for optimal combinatorial PMSG-based WT and RSFCL including optimal layer, criterion layer, and alternative layer.

Step 2) Compose a pairwise comparison matrix by assigning each alternative/criterion an optional number from 1/9 to 9 . In this article, the three-point performance rating scale is defined for the importance of criteria, 9 (high), 5 (medium), and 1 (low). Based on the explanation in [30], if the importance of criteria $C_{1}, C_{2}, C_{3}$ are ranked as high $\left(C_{1}=9\right)$, medium $\left(C_{2}=5\right)$, and low $\left(C_{3}=1\right)$, respectively, the criteria pairwise comparison matrix 


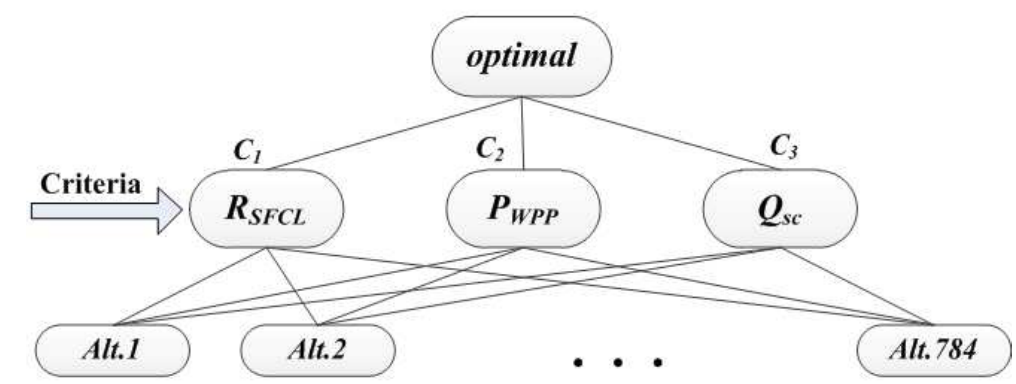

Figure 3.23: Hierarchy process for optimal combinatory PMSG-based WT and RSFCL.

$C=[c]_{3 \times 3}$ can be expressed by

$$
C=[c]_{3 \times 3}=\left[\begin{array}{ccc}
c_{11} & c_{12} & c_{13} \\
c_{21} & c_{22} & c_{23} \\
c_{31} & c_{32} & c_{33}
\end{array}\right]=\left[\begin{array}{lll}
C_{1} / C_{1} & C_{1} / C_{2} & C_{1} / C_{3} \\
C_{2} / C_{1} & C_{2} / C_{2} & C_{2} / C_{3} \\
C_{3} / C_{1} & C_{3} / C_{2} & C_{3} / C_{3}
\end{array}\right]=\left[\begin{array}{ccc}
1 & 9 / 5 & 9 \\
5 / 9 & 1 & 5 \\
1 / 9 & 1 / 5 & 1
\end{array}\right]
$$

A similar method is applied to estimate the value of alternative pairwise comparison matrix $A_{i}=\left[a_{i j}\right]_{784 \times 784},(i=1,2,3)$ with respect to each criterion.

Step 3) Compute the relative weight (priority) of the compared factor for the criterion according to the judgment matrix $C$ and $A$. The normalized criteria and alternatives weight vector can be obtained by adding the array elements of each row of $\mathrm{C}$ and $\mathrm{A}$ matrix and then dividing by the sum of the element of columns. Here, the weight vector matrix of criteria $w_{c j},(j=1,2,3)$ can be estimated by

$$
\begin{aligned}
{\left[\begin{array}{ccc}
1 & 9 / 5 & 9 \\
5 / 9 & 1 & 5 \\
1 / 9 & 1 / 5 & 1
\end{array}\right] \Longrightarrow\left[\begin{array}{c}
1+9 / 5+9=11.8 \\
5 / 9+1+5=6.56 \\
1 / 9+1 / 5+1=1.31
\end{array}\right] \Longrightarrow } \\
{\left[\begin{array}{l}
w_{c 1} \\
w_{c 2} \\
w_{c 3}
\end{array}\right]=\frac{1}{11.8+5.56+1.31}\left[\begin{array}{l}
11.8 \\
1.31
\end{array}\right]=\left[\begin{array}{l}
0.60 \\
0.334 \\
0.066
\end{array}\right] }
\end{aligned}
$$


The analysis of the simulation results represents the degree of importance of alternative $i$ in criterion $j$, i.e., $d_{i j}$, which is divided by its maximum value. This is followed by splitting the alternation range to nine parts, allocating a proportional number from 1 to 9 into each alternative $a_{i j}$ as

$$
a_{i j}=\operatorname{Integer}\left(\frac{d_{i j}}{0.11 M a x_{i}\left(d_{i j}\right)}\right)
$$

Since a pairwise comparison matrix of the alternative $A_{i}$ is compatible, it can form the calculation of the alternative weight vector simple via normalizing the elements of each column, reaching to $w a_{i j}$. These calculations can be formulated as

$$
w a_{i j}=\frac{a_{i j}}{\sum_{i=1}^{784} a_{i j}}
$$

The sum of the entire alternative weight vector with respect to the each criteria $w a_{i j}$ and the criteria weight vector wcj for $j=1,2,3$ and $i=1,2,, 784$, forms a decision matrix $D=[d]_{784 \times 3}$ as

$$
D=[d]_{784 \times 3}=\left[\begin{array}{ccc}
w a_{11} & w a_{12} & w a_{13} \\
w a_{21} & w a_{22} & w a_{23} \\
\cdot & \cdot & \cdot \\
\cdot & \cdot & \cdot \\
w a_{(784) 1} & w a_{(784) 2} & w a_{(784) 3}
\end{array}\right]
$$

Step 4) Calculate the best alternative, i.e., the highest priority value. Usually, the criteria can be classified into the two opposite groups called the benefit and cost criteria [160]. A benefit criterion means that the better alternative has the higher grade. The inverse scenario is expressed true for the cost criteria. In this optimization study, the total energy dissipated is cost and the other criteria, i.e., the resistance of SFCL and power output of the WPP, are benefit. Thus, the optimization problem can be summarized as a 


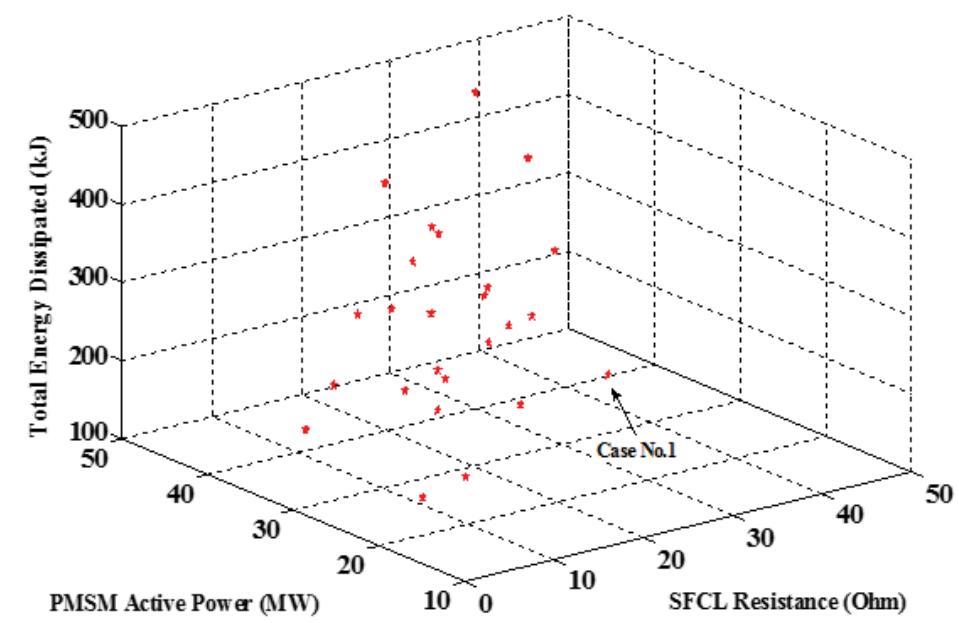

Figure 3.24: Multi-objective optimization using AHP, Pareto front for three criteria.

standard format for aggregating alternatives to rank them based on the ratio performance approach detailed in [160], as given by

$$
P_{A H P}^{*}=\max _{i}\left(\frac{\left(w a_{i 1} \times w_{c 1}\right)+\left(w a_{i 2} \times w_{c 2}\right)}{w a_{i 3} \times w_{c 3}}\right) \text { for } i=1,2,3, \ldots, 784 \text {. }
$$

For three levels criteria comparison, this weight vector must be calculated 25 times $\left(3^{2}-2\right)$ by changing the importance of the criteria with respect to each other. The run results of the algorithm are shown in Table 3.6. As earlier mentioned, the Pareto optimality plays the significant role in choosing the best solution for optimization of all three criteria: resistance of SFCL, energy dissipation in SFCL, and active power output of the WT. However, for an approximate set of three-dimensional Pareto-optimal solutions, a search is performed for the tradeoff values between the optimums of the objective functions, using AHP, at the end of each optimization run, as shown in Figure 3.25.

The corresponding AHP optimization results are illustrated in Table 3.6. It is the tradeoff values between the 25 given set of mutually contradicting criteria. Referring to Table 3.6, if higher priority is given to the RSFCL, so case 9 (H-L-L) in which $26.15 \Omega$ must be chosen. Similarly, for the power output of the PMSG-WPP or total energy dissipated priority selection, cases 20 and 24 (L-H-L and L-L-H) in which $P_{W P P}=28.59$ 
Table 3.6: Achieved Optimal Alternatives Using AHP Method.

\begin{tabular}{|c|c|c|c|c|c|c|c|c|c|c|}
\hline $\begin{array}{l}\text { Case } \\
\text { No. }\end{array}$ & Priority & $w_{c 1}$ & $w_{c 2}$ & $w_{c 3}$ & $V_{s c}\left(m^{3}\right)$ & $A_{s c}\left(m^{2}\right)$ & $\Delta t_{s c}(s)$ & $R(\Omega)$ & $P(M W)$ & $Q_{s c}(k J)$ \\
\hline 1 & $\mathrm{H}-\mathrm{H}-\mathrm{H}$ & 0.33 & 0.33 & 0.33 & $5.00 \mathrm{E}-04$ & $9.20 \mathrm{E}-07$ & 0.26 & 44.59 & 41.35 & 143.43 \\
\hline 2 & H-H-M & 0.39 & 0.39 & 0.22 & $4.30 \mathrm{E}-04$ & $1.04 \mathrm{E}-06$ & 0.32 & 29.54 & 44.75 & 200.95 \\
\hline 3 & H-H-L & 0.47 & 0.47 & 0.05 & $1.85 \mathrm{E}-04$ & $1.04 \mathrm{E}-06$ & 0.38 & 12.72 & 25.53 & 252.17 \\
\hline 4 & H-M-H & 0.39 & 0.22 & 0.39 & $4.65 \mathrm{E}-04$ & $1.04 \mathrm{E}-06$ & 0.38 & 31.94 & 40.94 & 234.51 \\
\hline 5 & H-M-M & 0.47 & 0.26 & 0.26 & $1.85 \mathrm{E}-04$ & $9.20 \mathrm{E}-07$ & 0.44 & 26.23 & 32.19 & 252.17 \\
\hline 6 & H-M-L & 0.60 & 0.33 & 0.07 & $1.85 \mathrm{E}-04$ & $1.04 \mathrm{E}-06$ & 0.44 & 19.72 & 20.82 & 291.99 \\
\hline 7 & H-L-H & 0.47 & 0.05 & 0.47 & $3.60 \mathrm{E}-04$ & $9.20 \mathrm{E}-07$ & 0.80 & 31.63 & 41.71 & 492.73 \\
\hline 8 & H-L-M & 0.60 & 0.07 & 0.33 & $3.25 \mathrm{E}-04$ & $9.20 \mathrm{E}-07$ & 0.74 & 27.53 & 31.31 & 466.48 \\
\hline 9 & H-L-L & 0.82 & 0.09 & 0.09 & $2.90 \mathrm{E}-04$ & $1.28 \mathrm{E}-06$ & 0.68 & 26.15 & 16.35 & 448.68 \\
\hline 10 & M-H-H & 0.22 & 0.39 & 0.39 & $3.95 \mathrm{E}-04$ & $9.20 \mathrm{E}-07$ & 0.26 & 34.67 & 43.42 & 155.92 \\
\hline 11 & M-H-M & 0.26 & 0.47 & 0.26 & $3.60 \mathrm{E}-04$ & $1.04 \mathrm{E}-06$ & 0.26 & 24.73 & 38.92 & 168.14 \\
\hline 12 & M-H-L & 0.33 & 0.60 & 0.07 & $5.00 \mathrm{E}-04$ & $1.40 \mathrm{E}-06$ & 0.32 & 18.95 & 23.22 & 211.95 \\
\hline 13 & M-M-H & 0.26 & 0.26 & 0.47 & $2.55 \mathrm{E}-04$ & $1.04 \mathrm{E}-06$ & 0.38 & 17.51 & 40.74 & 252.17 \\
\hline 14 & M-M-L & 0.45 & 0.45 & 0.09 & $3.25 \mathrm{E}-04$ & $1.52 \mathrm{E}-06$ & 0.5 & 10.45 & 29.36 & 331.38 \\
\hline 15 & M-L-H & 0.33 & 0.07 & 0.60 & $2.90 \mathrm{E}-04$ & $1.16 \mathrm{E}-06$ & 0.62 & 16.01 & 29.72 & 409.45 \\
\hline 16 & M-L-M & 0.45 & 0.09 & 0.45 & $3.60 \mathrm{E}-04$ & $1.52 \mathrm{E}-06$ & 0.68 & 11.57 & 25.89 & 448.68 \\
\hline 17 & M-L-L & 0.71 & 0.14 & 0.14 & $5.00 \mathrm{E}-04$ & $1.88 \mathrm{E}-06$ & 0.62 & 10.51 & 18.26 & 409.45 \\
\hline 18 & L-H-H & 0.05 & 0.47 & 0.47 & $4.30 \mathrm{E}-04$ & $1.76 \mathrm{E}-06$ & 0.2 & 10.31 & 39.31 & 131.39 \\
\hline 19 & L-H-M & 0.07 & 0.60 & 0.33 & $2.20 \mathrm{E}-04$ & $1.64 \mathrm{E}-06$ & 0.2 & 7.07 & 22.31 & 131.39 \\
\hline 20 & L-H-L & 0.09 & 0.82 & 0.09 & $1.50 \mathrm{E}-04$ & $1.16 \mathrm{E}-06$ & 0.26 & 8.28 & 28.59 & 231.62 \\
\hline 21 & L-M-H & 0.07 & 0.33 & 0.60 & $4.30 \mathrm{E}-04$ & $1.88 \mathrm{E}-06$ & 0.32 & 9.04 & 34.76 & 211.97 \\
\hline 22 & L-M-M & 0.09 & 0.45 & 0.45 & $2.90 \mathrm{E}-04$ & $1.64 \mathrm{E}-06$ & 0.38 & 8.01 & 25.25 & 252.17 \\
\hline 23 & L-M-L & 0.14 & 0.71 & 0.14 & $2.90 \mathrm{E}-04$ & $1.76 \mathrm{E}-06$ & 0.38 & 6.95 & 20.37 & 252.17 \\
\hline 24 & L-L-H & 0.09 & 0.09 & 0.82 & $5.00 \mathrm{E}-04$ & $1.88 \mathrm{E}-06$ & 0.74 & 10.51 & 30.22 & 128.92 \\
\hline 25 & L-L-M & 0.14 & 0.14 & 0.71 & $3.95 \mathrm{E}-04$ & $1.76 \mathrm{E}-06$ & 0.62 & 9.47 & 25.96 & 207.13 \\
\hline
\end{tabular}

MW and $Q_{s c}=128.92 \mathrm{~kJ}$ must be selected, respectively. Moreover, the higher and lower active power delivered during the fault are obtained in case 1 ( $82.7 \%$ of total capacity) and case $9(32.7 \%)$, respectively.

\subsection{Power Management of DC grid Involving PMSG WTs and SMES}

\subsubsection{Motivation and related works}

This section presents combinatory standalone VSWT-PMSG and micro-SMES system in order to stabilize the dc-link voltage, thereby smoothing the output power simultaneously. First, the desired energy storage capacity of the micro-SMES coil is precisely designated by some considerations, such as wind deviations and practical restrictions. This work was augmented by an optimization algorithm using the simulated annealing (SA) as a 


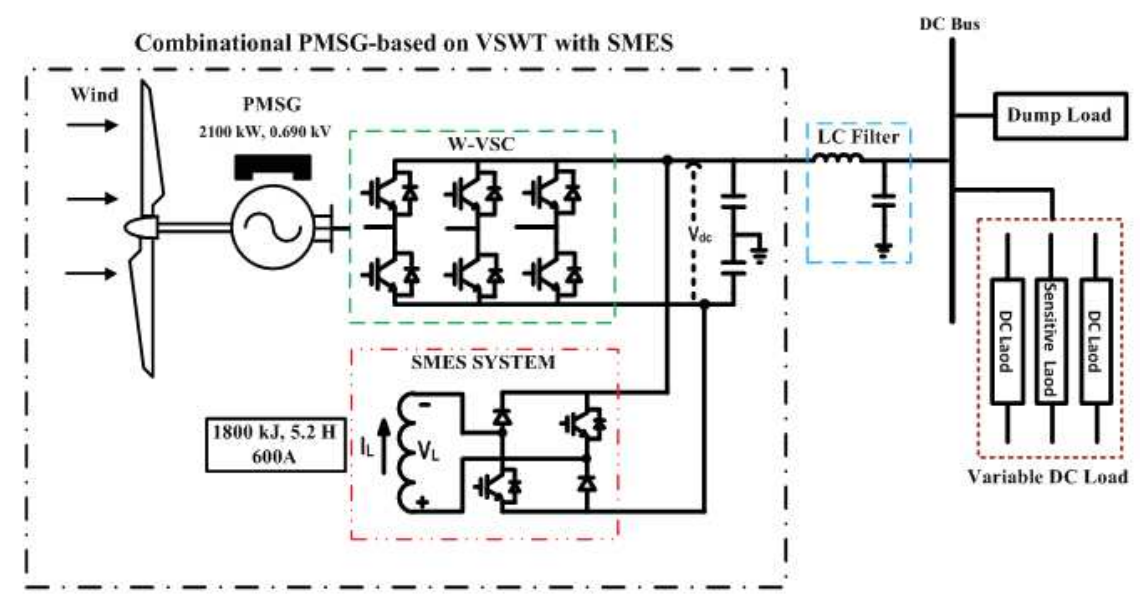

Figure 3.25: Combination of PMSG- based on VSWT and SMES system in dc microgrid.

conceivable technique for the optimized deign of the proposed SMES coil. Finally, this section represents the real-time energy management strategy for close coordination between the wind power, loads, and micro-SMES system, according to power and current measurement. For this purpose, the novel approach for the bi-directional DC-DC converter is expatiated corresponding an optimized charging and discharging model of the micro-SMES system.

\subsubsection{Overall System Configuration}

The proposed structure of the standalone dc microgrid system is schematically shown in Figure 3.26. A wind turbine using PMSG-based VSWT, rated at $1 \mathrm{MW}$ and $690 \mathrm{~V}$, is considered as a main source of the power generation. The generator-side converter including the three-phase voltage source converter (VSC), namely, W-VSC connected the PMSG to the dc network. The energy storage (ES) system, which is based on the small-scale SMES technology, is incorporated into the dc network through a bidirectional dc-dc converter, as shown in Figure 3.26.

An effective dump load is used to increase energy efficiency of the system by absorbing the excess power during the over wind generation. The surplus energy is employed 
in charging the SMES until current reaches its upper limit. After that, the extra power is supplied to a proper dump load for the heating purpose with the DC-DC converter. The total output power of wind turbine is supplied to the variable dc loads including directconnected and sensitive loads. During the prolonged operation in low wind mode, an appropriate load shedding must be considered in order to guarantee the system against total collapse and to cater a secure power supply to high-priority loads (sensitive loads).

\subsubsection{SMES System Description}

In this study, the micro-SMES system is implemented as a promising energy storage system for load levelling and power stabilizing in a standalone dc microgrid. Independent of the capacity and size, a SMES system constantly consists of a superconducting coil, which stores energy in the magnetic field generated by a circulating current, a cryogenic refrigerator to keep coil at a low temperature required to maintain it in a superconducting state, and a power conditioning system (PCS) in order to transfer the energy from the SMES coil into the grid [161].

\section{SMES System Capacity}

Although the balancing performance of the SMES coil can be increased by selecting a larger capacity, the cost installation of the SMES system will rise accordingly, making the implementation of the SMES unviable. Conversely, choosing an inadequate capacity for SMES system will most likely lead to an ineffective operation for minimizing wind power fluctuations. Thus, the coil capacity, which depends on its application and charging/discharging duration, must be carefully selected. For dc microgrid equipped by

VSWT-PMSG, if the micro-SMES balances the fluctuating power $\pm \Delta P_{\text {wind }}$ for a period of $\Delta t$ seconds, the SMES is used to compensate the stored energy, $\pm \Delta P_{\text {wind }} \times \Delta t$, duo to the intermittent wind fluctuations. 


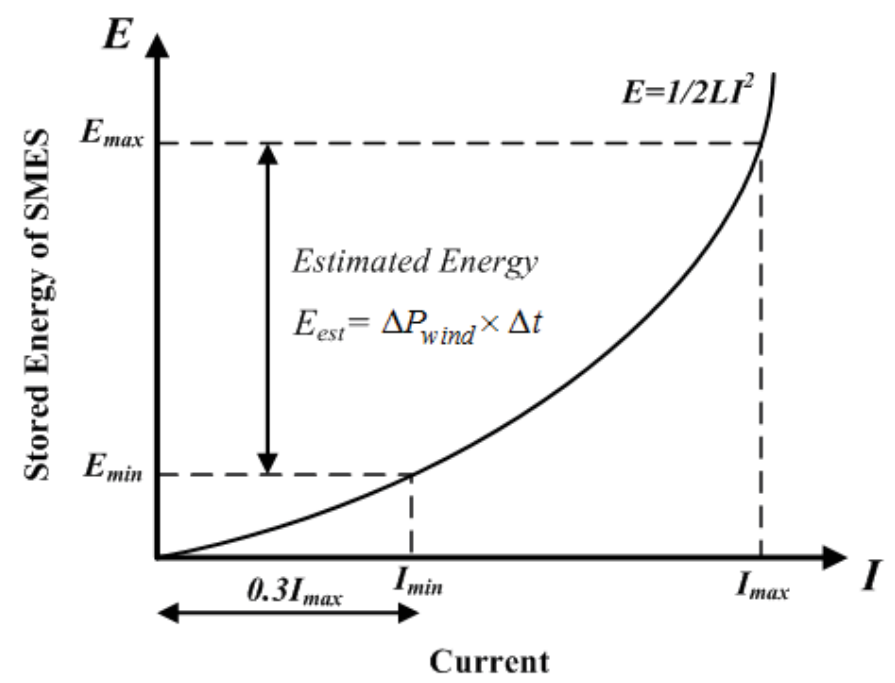

Figure 3.26: Estimated energy of the SMES according to lower and upper coil current limits.

As stated in (3.6), the wind power is proportional to the third power of wind speed. If the standard deviation of wind speed fluctuations is $20 \%$ of the average wind speed, the output power fluctuations will be about $80 \%\left(\Delta P_{\text {wind }}=800 \mathrm{~kW}\right.$ for a period of 1 second) of the rated $1 \mathrm{MW}$ from the wind power. Therefore, the capacity requirement of the SMES system can be estimated as the following

$$
E_{\text {est }}=\Delta P_{\text {wind }} \times \Delta t=800 k W \times 1 s=800 k J
$$

In practice, in order to prevent the possibility of discontinuous conduction under unpredicted disruptions, the SMES current $I_{\text {smes }}$, should not permitted to reach zero [45]. Figure 3.27 shows the relation between the stored energy of the SMES and the available energy for the wind turbine based on lower and upper coil current limits $\left(0.3 I_{\max }<\right.$ $\left.I_{\text {smes }}<I_{\max }\right)$. Here, $E_{\max }$ can be calculated from (3.28) and Figure 3.27, which has a relation as

$$
E_{\max }=\frac{E_{e s t}}{\left(1-0.3^{2}\right)}=880 k J
$$




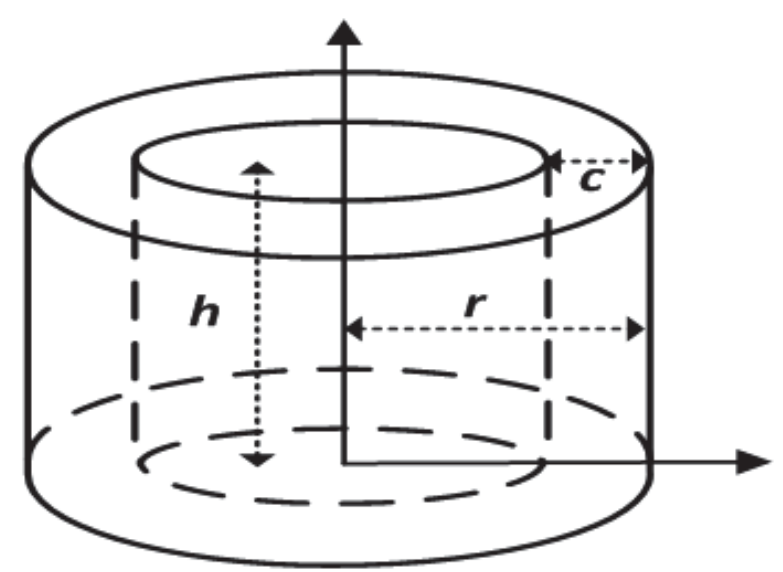

Figure 3.27: Cross section of multi-layer solenoid coil.

\section{Design of Micro-SMES Coil}

The magnet for micro-SMES coil usually is based on a low temperature superconductor (LTS) niobiumtitanium (NbTi) alloy. To reach the superconducting state, the coil has to be cooled to less than $4.20 \mathrm{~K}$ by liquid helium [43]. In this research, the coil was made of NbTi LTS tape, which was manufactured by the Furukawa Electric Co., Japan. The cross area of one superconducting filament is $2.07 \times 1.07 \mathrm{~mm}^{2}$, including insulation [162]. However, a SMES coil has been constructed in many different shapes but one of the most common kinds is the solenoid type winding, which are widely used because of their simple structure, feasibility to manufacture, and high efficiency. In order to facilitate the manufacturing a SMES coil with LTS tape, several turns of the coil arrangement can be assumed in a cylindrical fashion, as shown in Figure 3.28, where $h, r$, and $c$ are the height, the mean radius, and the radial thickness of the solenoid coil, respectively.

\section{Superconducting Coil Optimization}

An optimal design of a solenoid coil volume will influence the cost and the size of the overall SMES and thereby allow for the use of a smaller and more compact design. Thus, the coil volume is set as the objective function of this study for the optimal micro-SMES design. The considered objective function, i.e., minimum coil volume for the two con- 
straints, expected stored energy and allowable risk factor can be expressed as the following

$$
\begin{gathered}
\text { Min } V_{\text {coil }}=\pi\left\{(r+c / 2)^{2}-(r-c / 2)^{2}\right\} \times h \\
\text { Subject to }\left\{\begin{array}{l}
\frac{\left|E_{\text {smes }}-E_{\max }\right|}{E_{\max }} \leq \varepsilon \\
R_{f}=\frac{J}{J_{c}} \leq \gamma
\end{array}\right.
\end{gathered}
$$

where $E_{\text {smes }}$ is the energy actually stored in the coil, $E_{\max }$ is the expected stored energy $(880 \mathrm{~kJ}) . \varepsilon$ is the maximum relative error of $E_{\max }$, which is set as $1 \% . J$ is the current density in the coil, and $J_{c}$ is the critical current density of LTS tape, which usually depends on the maximum magnetic flux density $B_{\max }$ at the given temperature. $\gamma$ is the maximum allowable risk factor value and is assumed to be 0.7 . This optimization is accomplished by an algorithm based on simulated annealing (SA), which is a feasible method for an optimized design of the SMES solenoid.

SA is a method to obtain an optimal solution for a single-objective optimization problem. It is based on an analogy of thermodynamics with the way metals cool and anneal. If liquid metal is cooled slowly, its atoms form a pure crystal corresponding to the state of minimum energy for the metal. The metal reaches a state with higher energy if it is cooled quickly. This algorithm has the ability to escape from local minima and can deal with discrete variables $[21,43]$.

\subsubsection{Control Scheme in Standalone Mode}

The implemented control scheme used in the dc microgrid, including a W-VSC and DCDC chopper, is shown in Figure 3.29. The primary requirement for standalone operation mode is to maintain the common dc bus voltage within an acceptable range ( 0.95 pu and $1.05 \mathrm{pu}$ ) under any condition. Indeed, a constant dc voltage designates balanced active power flow among the wind power, SMES system and dc loads. 

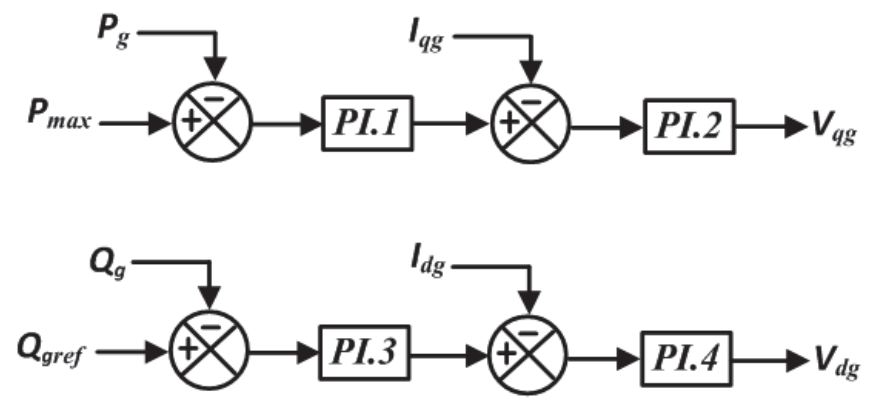

Figure 3.28: Control diagram of PMSG-based variable speed wind turbine.

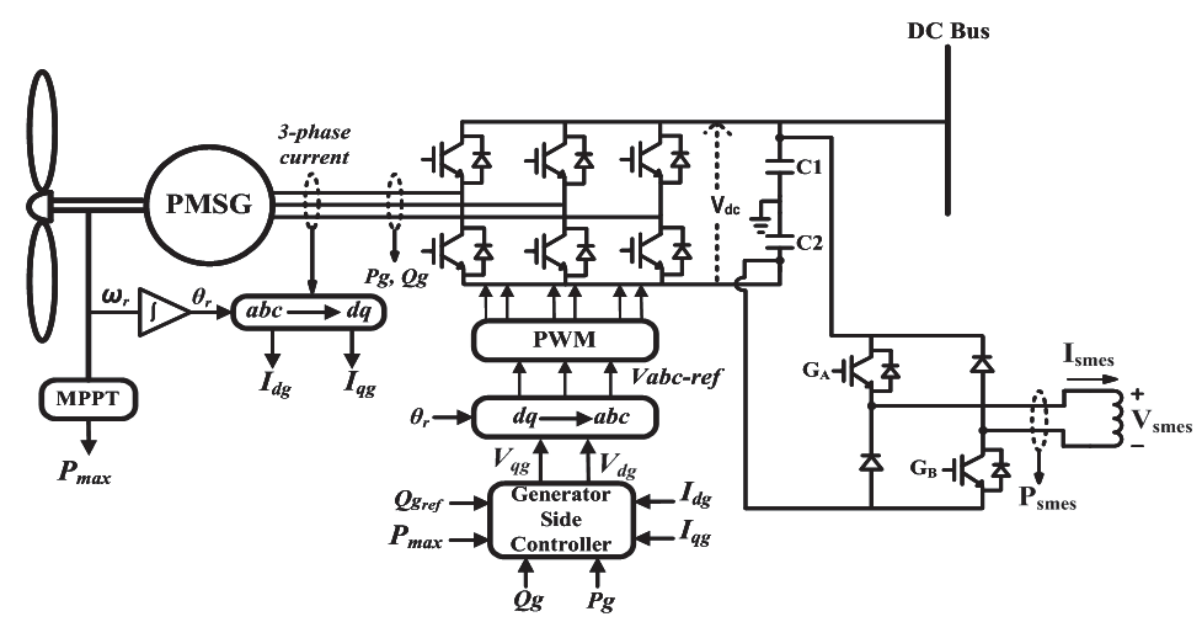

Figure 3.29: Generator side controller of PMSG.

\subsubsection{VSC Converter Control}

The voltage source converter consists of six IGBT switches interconnected as a full-bridge converter that is directly connected to the stator of the PMSG. The control block diagram for VSC converter, shown in Figure 3.30, is based on the synchronous $d-q$ reference frame. The active power reference, $P_{\max }$, is determined from MPPT in such a way, as pointed out previously. Also, the reactive power produced by the wind turbine is regulated at zero MVar $\left(Q_{\text {gref }}=0\right)$ under normal operating conditions. In the W-VSC converter, the triangle signal is used as the carrier wave of PWM operation. The carrier frequency is chosen $10 \mathrm{kHz}$ for the VSC converter. The dc-link capacitor value is $50000 \mu \mathrm{F}$, while the rated dc-link voltage across the two capacitor legs is $1.2 \mathrm{kV}$. 


\section{Two-Quadrant DC-DC Converter Control}

The micro-SMES coil is interconnected onto the dc link through a dc-dc bi-directional converter to facilitate an active power flow in both directions, as shown in Figure 3.29. It is assumed to be fully charged at its maximum capacity of $880 \mathrm{~kJ}$. The two IGBTs, $G_{A}$ and $G_{B}$, are controlled using the hysteresis band current control technique. The control of the IGBTs is carried out by comparison of generating power with the wind turbine, $P_{\text {wind }}$ and power consumed by the load, $P_{\text {load }}$. In order to maintain system stability, the micro-SMES system must be charged or discharged corresponding to the necessary power balancing as

$$
P_{\text {smes }}^{*}=P_{\text {wind }}-P_{\text {load }}
$$

where superscript $*$ represents the required (reference) value. For the acceptable operation of the dc microgrid during the variations of wind generation, three regions of operation can be identified for the dc-dc converter.

Standby mode: it takes place when output power of the wind turbine is equal to the load power $\left(P_{\text {smes }}^{*}=0\right)$. The SMES coil current is kept constant at its rated value. Consequently, there will be no energy transferred between the micro-SMES system and de microgrid.

Discharging mode: it will occur when output power of the wind turbine is smaller than the rated power $\left(P_{\text {smes }}^{*}<0\right)$. In this case, the required power is being delivered to the dc link capacitor with the dc-dc converter.

Charging mode: it will occur when output power of the wind turbine is greater than the rated power $\left(P_{\text {smes }}^{*}>0\right)$. Under this condition, the surplus of the wind power is being transferred to the SMES coil. For a dc microgrid system, required energy during power imbalance and $I_{\text {smes, } 1}$, SMES coil current at end of the exchanging period, $T$ is given as

$$
E_{\text {smes }}^{*}=\int_{T} P_{\text {smes }}^{*} d t
$$




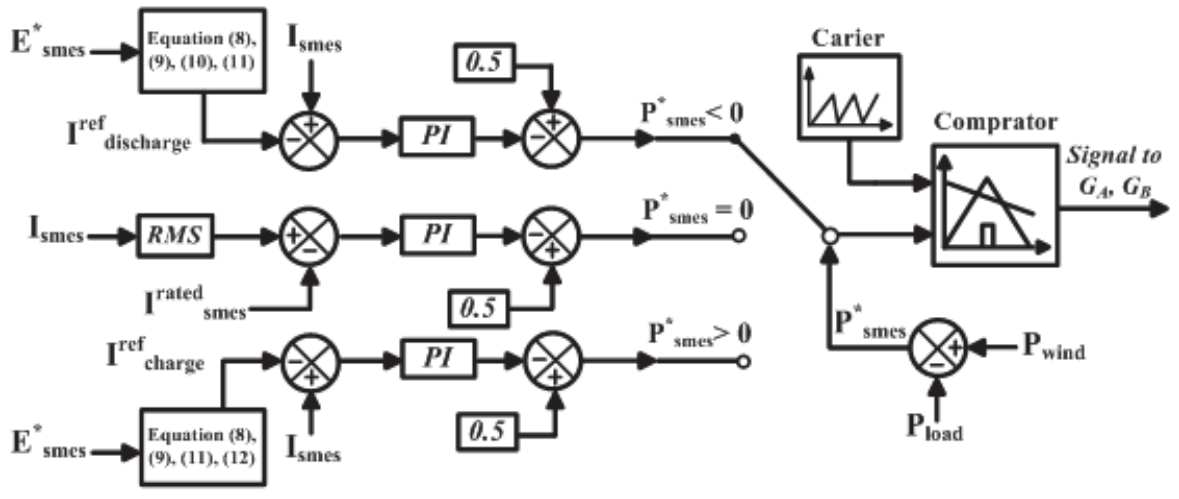

Figure 3.30: Control diagram of dc-dc converter with SMES coil.

$$
I_{s m e s, 1}=\sqrt{I_{s m e s, 0}^{2}-\frac{2 E_{s m e s}^{*}}{L_{s m e s}}}
$$

where $E_{\text {smes }}^{*}$ is energy exchange between micro-SMES system and dc link $(\mathrm{J}), L_{\text {smes }}$ is the coil inductance $(\mathrm{H})$ while $I_{s m e s, 0}$ represents the coil current at the beginning of the exchanging period. In this effort, it is assumed that the superconducting coil is being charged or discharged based on an exponential function shown by the following equations

$$
\begin{gathered}
I_{\text {discharge }}^{r e f}=I_{\text {smes }, 0} \times e^{-\alpha\left(t-t_{d}\right)} \\
I_{\text {charge }}^{\text {ref }}=-I_{\text {smes }, 1} \times e^{-\beta\left(t-t_{d}\right)} \\
\alpha=\beta=\frac{1}{T} \times \ln \left(\frac{I_{\text {smes }, 0}}{I_{\text {smes }, 1}}\right)
\end{gathered}
$$

The proper operation of the control system is occurring when coil current pursues these reference currents during the charging/discharging operation mode. In order to generate the gate signals for the $G_{A}$ and $G_{B}$, the reference duty cycle signals must be compared with the carrier with the frequency of $4 \mathrm{kHz}$. The schematic of the dc-dc converter controller used to regulate the current of the SMES to maintain the dc bus voltage constant is depicted in Figure 3.31. 


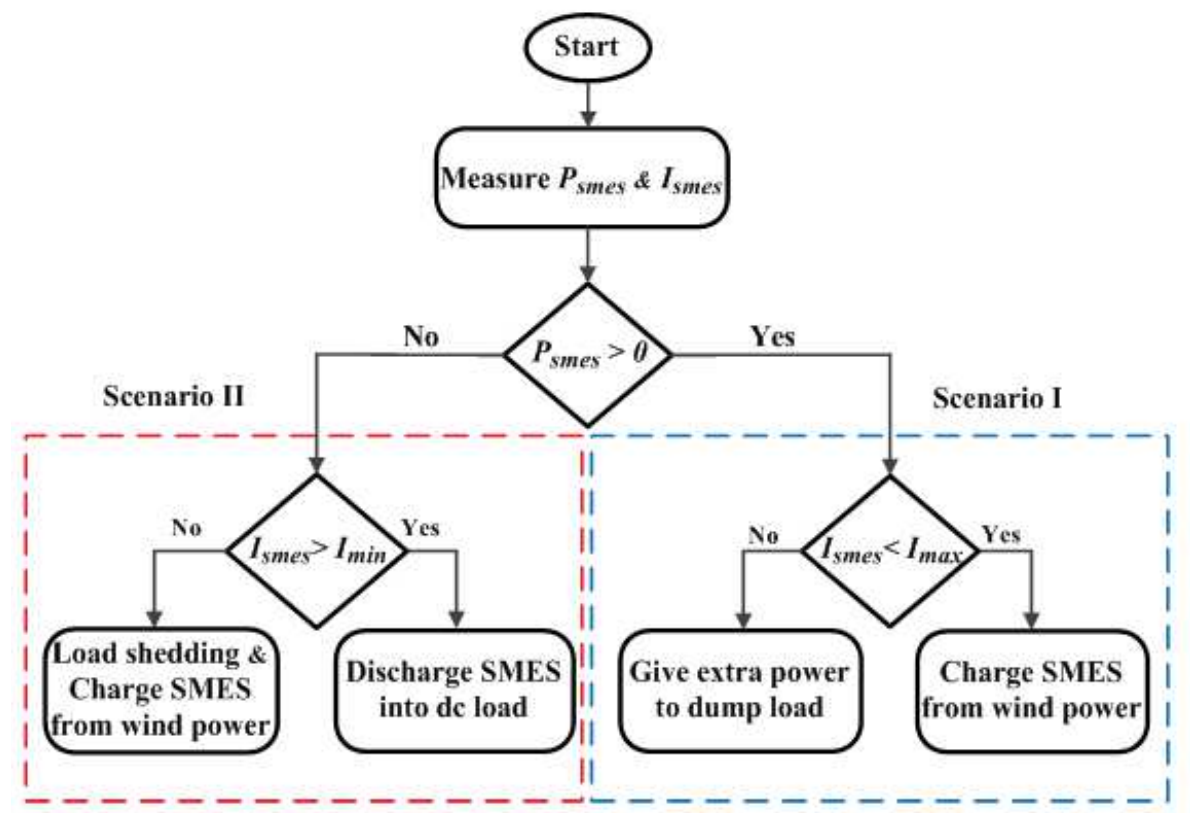

Figure 3.31: Energy management strategies for the standalone DC Microgrid.

\section{Real-Time Energy Management System}

A comprehensive load management strategy is introduced in wind power and photovoltaic are considered as smart agents, which are utilized for distribution network load management [163]. Another effective means for energy management is demand response programs utilization considering the high penetration of plug-in hybrid electric vehicles [164]. In order to optimize energy utilization specifically during a long-term lowwind or high-wind period, an appropriate real-time power management must be incorporated into the control system to maintain the dc bus voltage constant. The flowchart for the suggested energy management algorithm, which accounts for two scenarios, is annotated in Figure 3.32.

Scenario 1: if the maximum available power from the VSWT-PMSG is greater than demand, the excess power $\left(P_{s m e s}^{*}\right)$ can be used to charge the SMES coil until current reaches its upper limit $\left(I_{\max }\right)$. In this case, SMES system is set in standby mode and extra power is supplied to a proper dump load. However, this condition is not considered in the 
study.

Scenario 2: In cases where power from the wind turbine is not enough to meet the demand, the SMES needs to be discharged in order to prevent the total voltage collapse of the dc microgrid. Similarly, if SMES coil current exceed lower limit $\left(0.3 I_{\max }\right)$, the nonsensitive load will be automatically disconnected and the micro-SMES system will switch its control from discharging to charging mode to absorb extra wind power according to latest reduced demand.

\subsubsection{Simulation Results and Discussion}

In this section, simulation studies are carried out to verify the effectiveness of the standalone dc microgrid using PMSG-based wind turbine and micro-SMES system, as shown in Figure 3.26. The evaluation technique described in Section 3.4.4 is applied to the dc microgrid to enhance system efficiency from viewpoints of cost cutting, energy savings, and downsizing.

\section{Optimal Scheme Performance}

A simulated annealing (SA) method is employed for the design of an optimal SMES solenoid coil. The optimization solution is obtained by performing a certain number of iterations, leading to an optimal design. The constraints of the selected variables for the optimization problem are expressed as the following

$$
\left\{\begin{array}{l}
r \in[400,600] \mathrm{mm} \\
c \in[100,200] \mathrm{mm} \\
h \in[200,400] \mathrm{mm}
\end{array}\right.
$$

Figure 3.33 shows the progression of the solution of the optimization problem, whereas the objective concentrates on an optimal point, $V_{\text {coil }}=0.105 \mathrm{~m}^{3}$, during cooling in the annealing process. The optimized coil size and corresponding SMES coil inductance are 


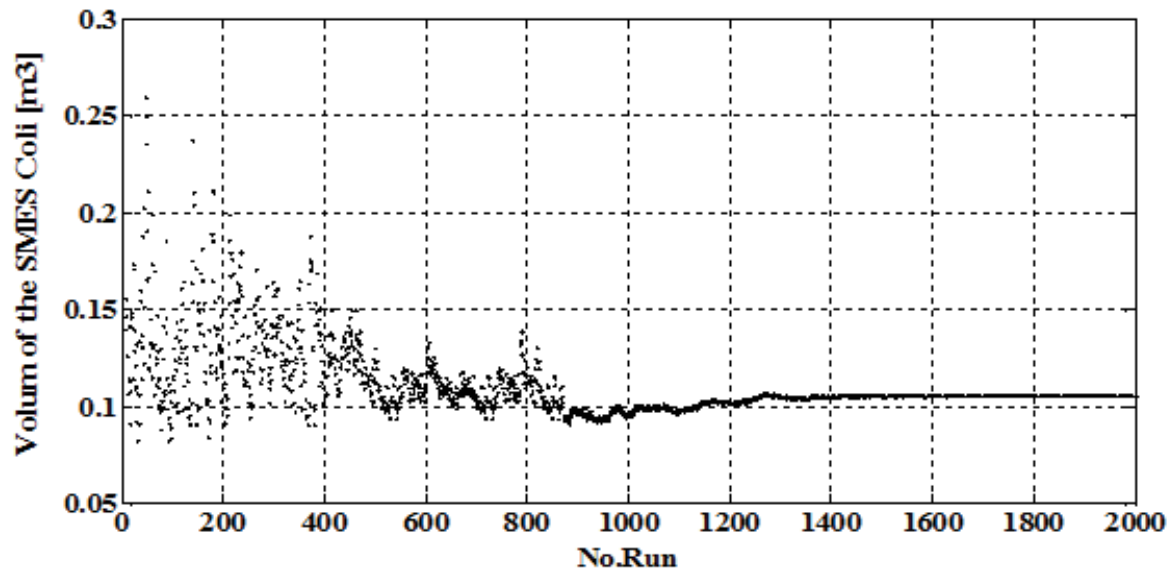

Figure 3.32: Progress of the solution of the proposed optimization considering $V_{\text {coil }}$ as an objective function.

Table 3.7: Optimization Results of The SMES Coil.

\begin{tabular}{|l|l|}
\hline Parameter & Quantity \\
\hline Height of the coil $h$ & $265 \mathrm{~mm}$ \\
Mean radius of the coil $r$ & $438 \mathrm{~mm}$ \\
Radial thickness of the coil $c$ & $150 \mathrm{~mm}$ \\
Inductance of the coil $L$ & $7 \mathrm{H}$ \\
\hline
\end{tabular}

shown in Table 3.7. According to the above results, the maximum current flowing through the coil at a steady state can be obtained

$$
I_{\text {max }}=\sqrt{\frac{2 E_{\max }}{L_{\text {coil }}}=500 \mathrm{~A}}
$$

\section{Simulation Case Studies}

The proposed system is modeled and simulated by PSCAD/EMTDC software. As can be seen from Figure 3.26, it is made up of the PMSG-based wind turbine rated at $1 \mathrm{MW}$ and $690 \mathrm{~V}$ and an $1 \mathrm{MW}$ W-VSC with switching frequency $10 \mathrm{kHz} . L_{l}$ and $L_{2}$ are constant power loads, both rated at $330 \mathrm{~kW}$, while $L_{l}$ (sensitive load) is the most important load and cannot be switched out during operation. For a wind speed of $11 \mathrm{~m} / \mathrm{s}$, the wind 


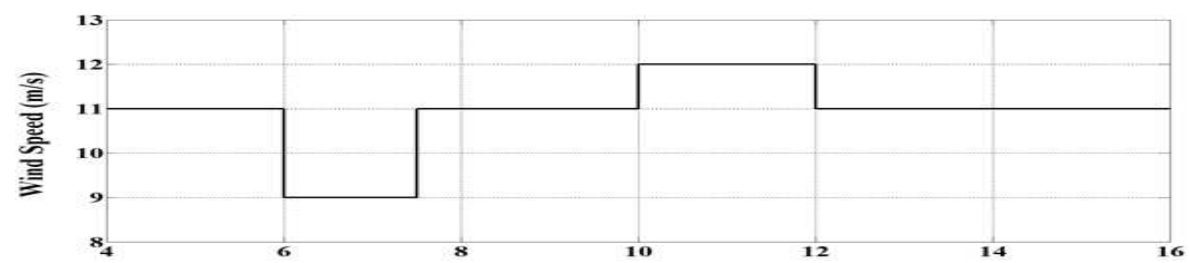

(a)

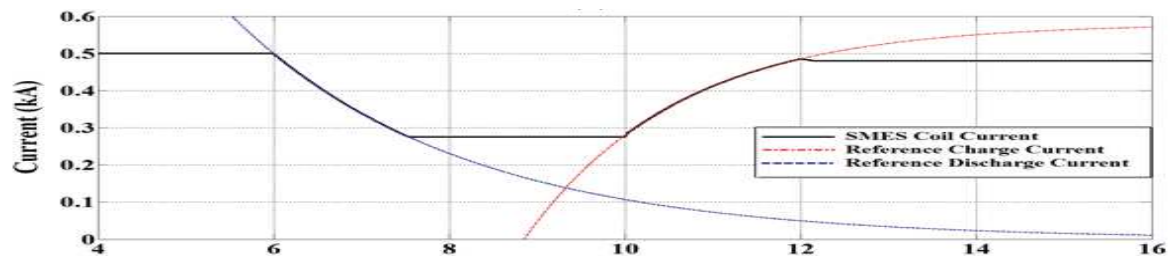

(b)

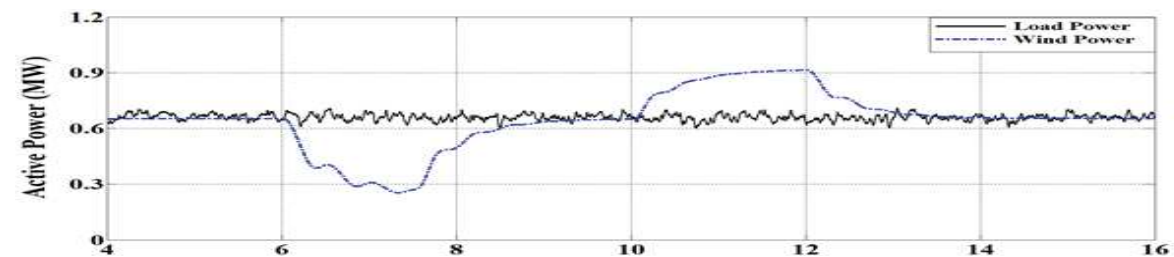

(c)

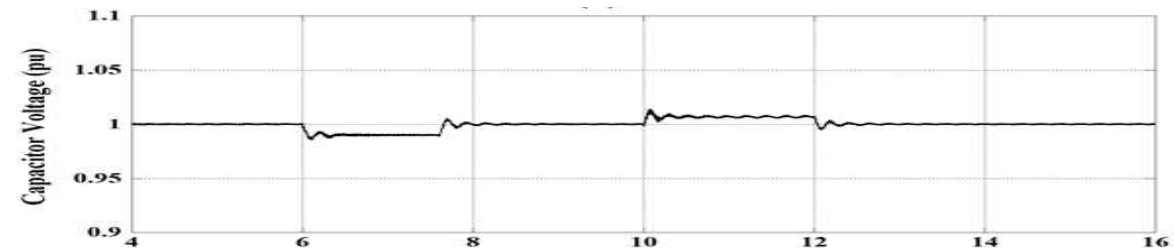

(d)

Figure 3.33: System operation during normal charging/discharging mode (Case 1). (a) Wind speed. (b) SMES coil current. (c) Power generated by the wind turbine and total load. (d) DC-link voltage.

turbine delivers rated power $660 \mathrm{~kW}$ to the loads. The micro-SMES system, consisting of the superconducting coil with capacity of $880 \mathrm{~kJ}$ and the bi-directional dc-dc converter, is connected to the $1.2-\mathrm{kV}$ bus via the dc-link capacitor. To ensure that the proposed micro-SMES operates properly, the supervisory control architecture previously outlined is analyzed by considering the following cases:

\section{Case 1: Normal Charging/Discharging Operation}

Figure 3.36 depicts simulation results for Case 1, including normal charging and discharging operation. The simulation starts in wind speed of $11 \mathrm{~m} / \mathrm{s}$ and $L_{1}, L_{2}$ are switched 
in $\left(P_{\text {load }}=660 \mathrm{~kW}\right)$, as shown in Figure 3.36(a) and Figure 3.36(c), respectively. The micro-SMES coil fully charges at its maximum current of 500 A. At $t=6 s$, the wind speed changes to $9 \mathrm{~m} / \mathrm{s}$ and wind turbine power reduces to $290 \mathrm{~kW}$, which is less than demand, thus the required power, $370 \mathrm{~kW}$, must be supplied by the micro-SMES system to keep load power constant at $660 \mathrm{~kW}$ (discharging mode). The micro-SMES current is reduced to approximately $282 \mathrm{~A}$ as a result of the discharging mode as shown in Fig. 11 (b).

At around $t=7.5 \mathrm{~s}$, wind speed comes back to $11 \mathrm{~m} / \mathrm{s}$ and the SMES current remains in standby mode, drawing no power for supplying power to the dc microgrid. At $10 \mathrm{~s}$, the wind speed goes up to $12 \mathrm{~m} / \mathrm{s}$ and subsequently does the generated power, $900 \mathrm{~kW}$, as seen in Figure 3.34(c). The excess power, $340 \mathrm{~kW}$, must be drawn by the micro-SMES system to keep the dc voltage less than 1.05 p.u. Under this condition, coil current rises from 282 A to 485 A and the micro-SMES capacity will be again set at around the maximum amount. The charging and discharging currents exactly track the reference currents, as shown in Figure 3.34(b). Obviously, it is found that the performance of the proposed control method is entirely acceptable. The output dc-link voltage at the constant load is presented in Figure 3.34 (d), which is well controlled by the micro-SMES system.

Case 2: Long-Term Low-Wind Operation Simulation results of system control and operation corresponding to Case 2 are shown in Figure 3.35. Similarly, at the first of simulation, all the situations are the same with before case between $4 \mathrm{~s}$ and $6 \mathrm{~s}$. At $6 \mathrm{~s}$, the wind speed drapes from $11 \mathrm{~m} / \mathrm{s}$ to $10 \mathrm{~m} / \mathrm{s}$ for a period of $2 \mathrm{~s}$. In this condition, the micro-SMES system increases its power output to compensate this in order to maintain the dc-link voltage at 0.97 p.u. [Figures 3.35(a) and (d)]. At around 9 s, the micro-SMES current is reduced to $150 \mathrm{~A}$ which is the equal to threshold value $\left(0.3 I_{\max }\right)$. Under this condition, the system tries to reserve energy for the most important load (i.e., $L_{1}$ in this example). Thus, $L_{2}(330 \mathrm{~kW})$ is tripped and the SMES can now start to get charged as 
can be seen from Figure 3.37(b) as the wind turbine generates more power than the latest reduced demand $\left(L_{1}\right)$. After tripping $L_{1}$ for $1 \mathrm{~s}$, at $10 \mathrm{~s}$, the wind speed increases to 12 $\mathrm{m} / \mathrm{s}$ and the micro-SMES gradually gets charged to roughly $860 \mathrm{~kW}$ and $L_{2}$ reconnect to the microgrid, as shown in Figure 3.35(c), while at the end of this period coil current reach 375 A [see Figure 3.35(b)].

At $\mathrm{t}=12 \mathrm{~s}$, wind speed comes back to $11 \mathrm{~m} / \mathrm{s}$ to provide total load $(660 \mathrm{~kW})$. Finally, at $14 \mathrm{~s}$, the wind speed decreases again to $10.5 \mathrm{~m} / \mathrm{s}$ and wind turbine power reduces to $545 \mathrm{~kW}$, which is less than demand. Thus, the required power, $115 \mathrm{~kW}$, must be provided by the micro-SMES system. As seen from Figure 3.35(d), the dc-link voltage is well controlled during various operating conditions, and the proposed control strategy is satisfactory.

\subsection{Economic and Practical Feasibility of the RSFCL}

Since the discovery of high temperature superconductors (HTS) in 1987, their immense potential to improve the way in which electrical energy could be efficiently generated, transmitted, transformed, used, and stored has been well recognized. Abundant technological achievements have been made in developing brittle HTS materials in a tape/wire form in lengths of about a kilometer to transport about three hundred times the current of copper. HTS tape processing involves thin film deposition of a multilayer stack on nickel alloy tapes. In order to achieve high critical currents, the superconducting film is grown epitaxially in a single-crystalline-like form on buffer layers which provide a single crystalline-like template even when deposited on a polycrystalline metal substrate.

The requirements for thin film HTS tapes in SFCLs are very stringent. A major factor limiting a significant commercial deployment of SFCLs is the deficiencies in the thin film HTS tape itself. One of the major challenges involved in realization of a practical FCL device is the ability of the device to respond to thermal transients. The dilemma is that 


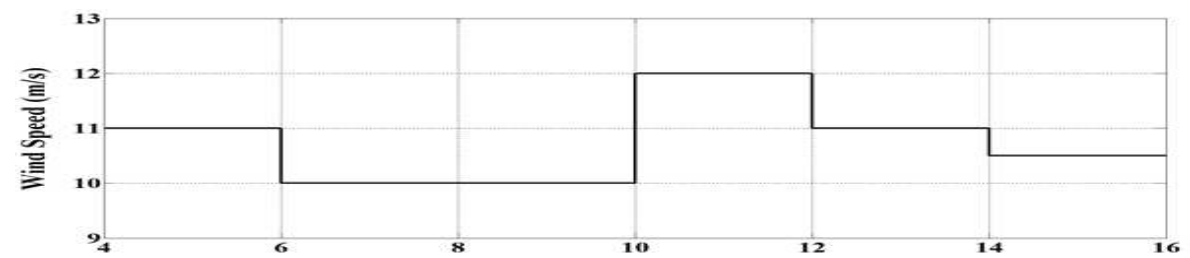

(a)

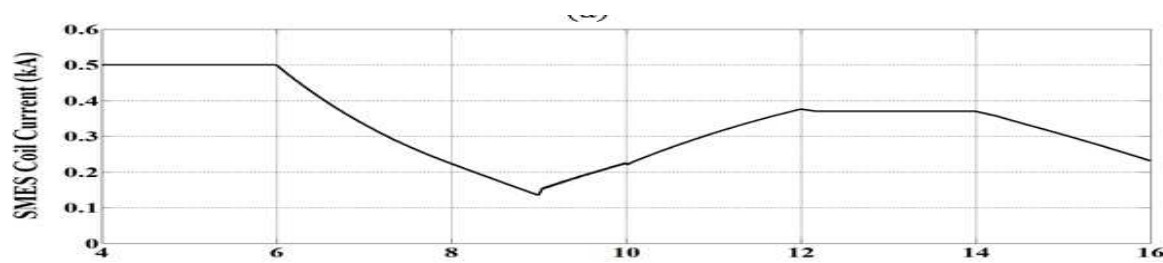

(b)

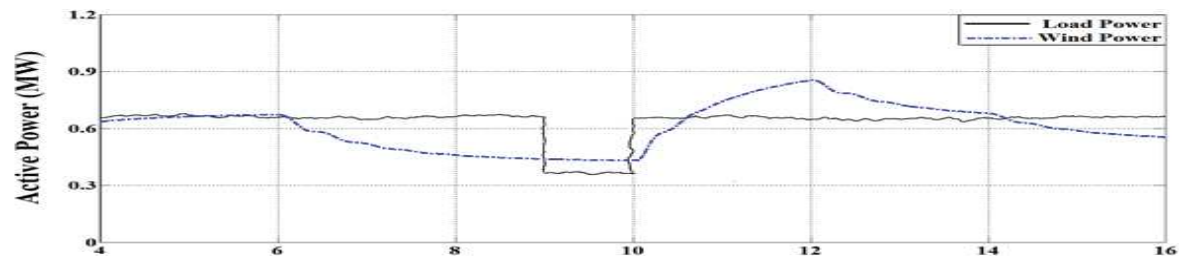

(c)

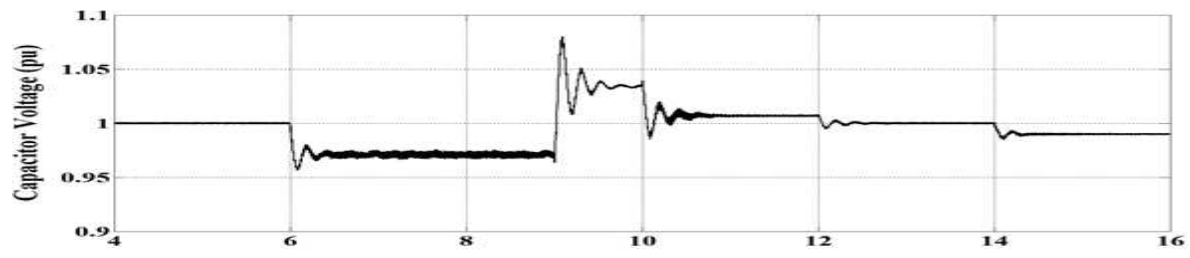

(d)

Figure 3.34: System operation during prolonged low-wind mode (Case 2). (a) Wind speed. (b) SMES coil current. (c) Power generated by the wind turbine and total load. (d) DC-link voltage.

the metallic constituents of the HTS conductor for providing good thermal conductivity cannot be substantially increased for the sake of not drastically increasing the amount of conductor needed to achieve a specified voltage drop $(\mathrm{V} / \mathrm{cm})$. So, there is a need for modifying the thin film HTS structure to provide better heat dissipation during the thermal transients without significantly altering the electrical properties of the device.

A major factor limiting a significant commercial deployment of SFCLs is the deficiencies in the thin film HTS tape itself. The following critical deficiencies in the thin film HTS tape need to be eliminated: (i) Non-uniform quench (ii) Low voltage drop (iii) Slow heat removal. Industries manufacturing thin film HTS tapes have made only incremental 


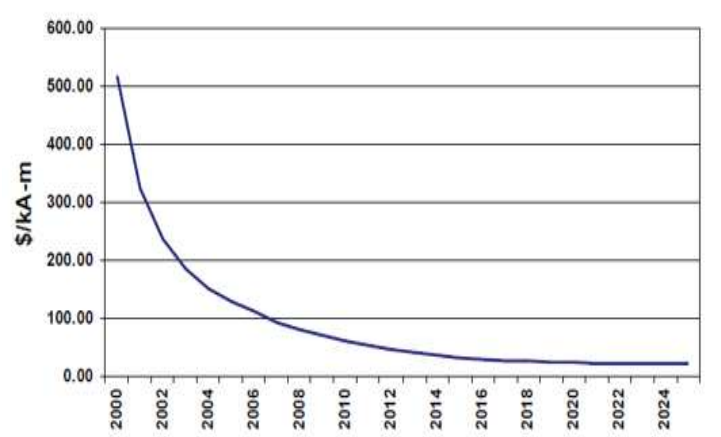

(a)

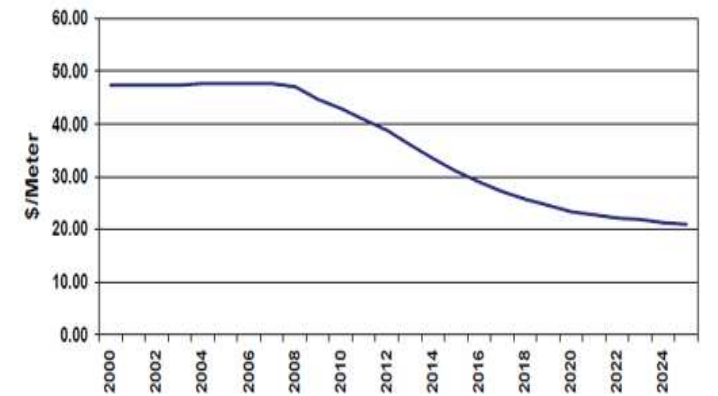

(b)

Figure 3.35: HTS wire cost. (a) Dollar per Kilo amp per Meter. (b) Dollar per Meter.

improvements in the tapes for SFCLs. A challenge has been a lack of understanding of the processes during the very rapid transition from superconducting to a resistive state during a fault and during recovery back to the superconducting state.

Several main factors affect for determining the actual size and cost of a resistive SFCL such as the length of applied superconducting wire, cooling machinery, the geometry of RSFCL module, and the rated power and voltage system where RSFCL must be installed. Practically, the whole superconducting length is used in form of helix to shape the superconducting tube. In reality, several tubes may be connected in parallel to achieve a particular resistance in form of cylindrical geometry. The rough estimation for the RSFCL size can be achieved based on design details of RSFCL projects worldwide [165]. Accordingly in this section, the RSFCL module installed in transmission system with voltage rate of $34.5 \mathrm{kV}$ and power rate of 50 MVA would be much less than $4 \mathrm{~m}$ in both diameter and height. After recent progress of economical second-generation HTS wires, SFCLs are becoming more practicable, because of low manufacturing costs, low ac loss, higher current density and better operational performance, and is eventually expected to be at least a factor of ten lower in cost than presently available HTS conductor [36]. The cost of HTS wire is generally described by two parameters: the maximum amount of current that the HTS wire can conduct; and the manufacturing cost per meter of wire. Figure 
3.16 illustrates how the HTS wire cost of RSFCL is expected to decrease over the next two decades as production increases. The impact of cooling system on the future competitiveness of RSFCL devices is critical. The 1999 benchmark cost of a medium-sized cryogenic refrigeration unit was about $\$ 60,000 / \mathrm{kWcold}$ at $77 \mathrm{~K}$. Economies of scale typical of the cooling refrigeration industry were applied to represent the expected decline in refrigeration costs. This declining cost model indicates that, as large numbers of cryogenic refrigeration units are manufactured, the cost will drop to less than $\$ 20,000 / \mathrm{kW}$ cold.

\subsection{Summary}

This chapter has been divided into three major studies. Section 3.2 has described the UPQC system to improve the power quality, thereby enhancing low voltage ride-through capability of the FSIG-based wind turbine. UPQC has the advantages of series and shunt APFs for compensating the distortions of grid voltages and load currents. Moreover, the application of the RSFCL, in providing an additional low voltage ride-through support to the wind-driven FSIG, has been investigated. By using the RSFCL, the grid current has been limited effectively and the minimum voltage level at the generator terminal has been increased, leading to a compliance with international grid codes. Additionally, the overall dynamics of FSIG, represented by active and reactive power, have been improved by RSFCL. The results show that the integration of the FSIG-based wind turbine and UPQC system will become more promising from the energy-saving and downsizing perspective by introducing the RSFCL as a self-healing limiter.

Section 3.3 has proposed an effective approach using RSFCL as the additional support, along with a conventional converter control strategy based on the PI controller to further increase the rated active power of the installation, thereby enhancing dc-link voltage smoothness, as well as the LVRT capability of the 50 MW WPP. Moreover, it has been demonstrated that the RSFCL can be a promising solution for improving the wind 
turbine controller performance with respect to extreme loads on the wind turbine structure. With this approach, it is expected that the activation of the dc braking chopper and fast pitch angle control could be reduced in order to meet the international grid code requirements. An important feature of the proposed method is that a conventional PI control can be used, performing the reactive and reactive current injection, while the dc-link voltage never exceeds its safety limits. A further study is carried out to determine the optimal performance of the combinatorial 50 MW PMSG-WPP and RSFCL. Therefore, a simultaneous and transformative approach based on the AHP method for the multiobjective optimization of embedded system has been introduced. A reconciliation between the three objecting functions, namely, resistive of SFCL, output power of PMSG, and energy dissipated in RSFCL was elicited by a 3-D alignment in the Pareto front, having 25 nondominated solutions. However, a designer would be capable of selecting any of the solutions setting on the Pareto front without erratic problems on optimality.

Finally, Section 3.4 has studied the micro-SMES system embedded to the VSWTPMSG, that is interconnected to the standalone dc microgrid through the bi-directional DC-DC converter. A power balance between loads, micro-SMES system and nondeterministic wind generation has been maintained via presenting the supervisory control architecture. For this purpose, an optimal design scheme based on the SA algorithm has been modeled for the micro-SMES solenoid coil to ensure the desired energy storage capacity $(880 \mathrm{~kJ})$ with the least volume. Moreover, this section presents an effective control strategy to obtain a rapid response of the micro-SMES system to the wind speed fluctuations in order to stabilize dc-link voltage, thereby smoothing the output power simultaneously. This penetrative approach allows researchers to find the optimized charging and discharging model based on the exponential function. System simulations corresponding to the aforementioned operational modes have confirmed the satisfactory manoeuvre of the proposed dc microgrid. 


\section{CHAPTER 4}

\section{IMPROVING OPERATIONAL RESILIENCE OF RENEWABLE ENERGY SYSTEM USING A STATCOM}

\subsection{Overview}

This chapter presents a computational intelligence technique for optimal coordinated reactive power control between a wind turbine (WT) equipped with doubly fed induction generator (DFIG) and a static synchronous compensator (STATCOM), during faults. The key motivation of this study is to design a coordinated reactive power controller that is formulated as a multi-objective optimization problem (MOP) model. By decreasing the level of the voltage deviations at the WT terminal, the low voltage ride-through (LVRT) capability of the WT can be significantly improved. Thus, one objective function considered in this study is the voltage deviations. This chapter also proposes an index called transient power severity index (TPSI) to quantify the transient active power performance of the system after the fault clearing time. The minimization of the TPSI for system buses is considered as the second objective function. The proposed two-objective optimization problem is solved by using the stochastic normalized simulated annealing (SNSA) algorithm in order to find the Pareto-optimal solutions, according to the assigned priorities (weights) for each objective. In order to minimize these two objective functions, it is necessary to find the optimal value of control variables, which are the reactive power references for both DFIG and STATCOM controllers. For the online coordinated reactive power control, the obtained optimal signals of the reactive power references are used in a fuzzy logic controller (FLC) for offline tuning the fuzzy model and fuzzy rules. To validate the effectiveness of the proposed control strategy for online applications, a case study including a 1.5-MW DFIG and a 1.5-MVar D-STATCOM were carried out with MATLAB/SIMULINK. 


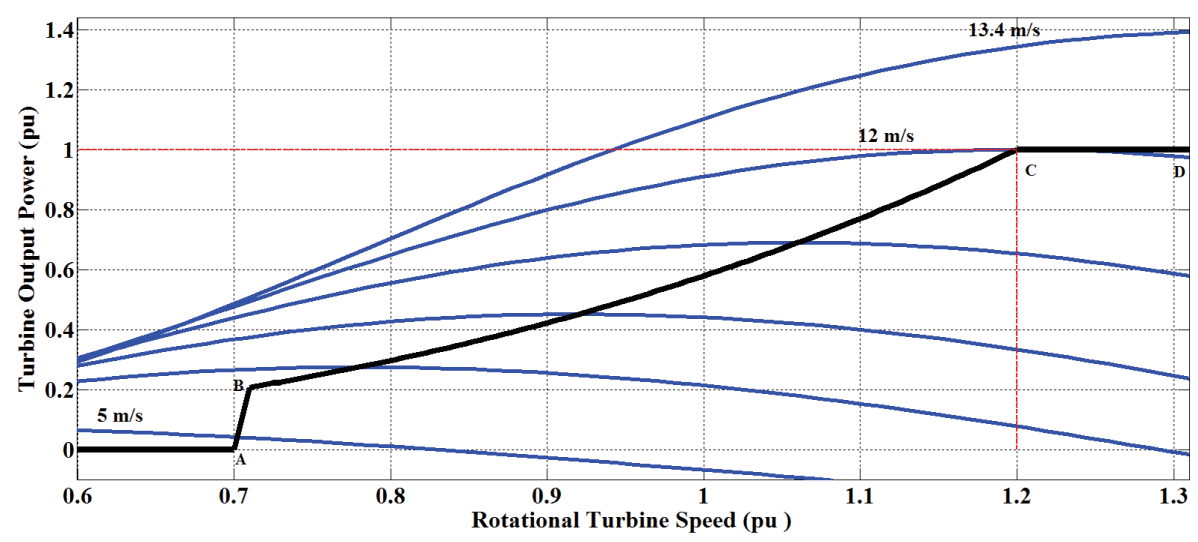

Figure 4.1: Wind turbine characteristics.

\subsection{DFIG Wind Farm Model}

\subsubsection{Wind Turbine Model}

According to the Betz theory, extractable mechanical power from the wind can be determined by the mathematical relation, as follows [13]

$$
P_{m}=\frac{1}{2} \rho A v_{w}^{3} C_{p}(\lambda, \beta)
$$

where $\rho$ is the air density, $A$ is the blade swept area, $v_{w}$ is the wind speed, and $C_{p}$ is the power conversion coefficient, which is a function of both tip-speed ratio $\lambda$ and the blade pitch angle $\beta$, in which $\lambda$ is defined by

$$
\lambda=\frac{\omega_{t} R}{v_{w}}
$$

where $R$ is the radius of the blade and $\omega_{t}$ is the rotational turbine speed. The $C_{p}(\lambda, \beta)$ curves depend on the blade design and are given by the wind turbine manufacturer. Based on wind turbine characteristics shown in Figure 4.1, the blades start to move around 5 $\mathrm{m} / \mathrm{s}$ at rotational speed 0.7 p.u (zone A-B, slip of the generator is $s=+0.3$ ). For base wind speed of $12 \mathrm{~m} / \mathrm{s}$, the maximum turbine output power $1 \mathrm{p} . \mathrm{u}$ at rotational speed $1.2 \mathrm{p} . \mathrm{u}$ are obtained (i.e. slip of the generator is $s=-0.2$ ). If the wind speed is below the rated value (zone B-C), the wind turbine operates in the variable-speed mode, and the rotational 
speed is adjusted (by means of increasing slip in the DFIG) such that the maximum value of $C_{p}$ is achieved. The extra power obtained from rotational speed higher than 1.2 p.u may be smoothly curtailed by spinning the blades using pitch angle control (zone C-D).

\subsubsection{Drive Train Model}

From the transient point of view, the drive train model must be presented to describe the performance of the WT in active and reactive power generation. In this effort, the twomass lumped model is utilized to designate mechanical dynamics, as proposed in [14]

$$
\left\{\begin{array}{l}
2 H_{t} \frac{d \omega_{t}}{d t}=T_{t}-D_{t} \omega_{t}-D_{s h}\left(\omega_{t}-\omega_{r}\right)-T_{s h} \\
2 H_{g} \frac{d \omega_{r}}{d t}=T_{s h}-D_{g} \omega_{r}+D_{s h}\left(\omega_{t}-\omega_{r}\right)-T_{e} \\
\frac{d T_{s h}}{d t}=K_{s h}\left(\omega_{t}-\omega_{r}\right)
\end{array}\right.
$$

where $\omega_{t}$ and $\omega_{r}$ are the turbine and generator rotor speed; $T_{m}, T_{e}$, and $T_{s h}$ are the mechanical torque applied to the turbine, the electrical torque of the generator, and internal torque of the model; $H_{t}$ and $H_{g}$ are the inertia constants of the turbine and the generator; $D_{t}, D_{g}$, and $D_{s h}$ are the damping coefficients of the turbine, the generator, and the flexible coupling (shaft) between the two masses; and $K_{s h}$ is the shaft stiffness.

The low-frequency oscillations of the output active power due to power unbalance during the fault can be lightly damped by the WT shaft system described by (4.3).

\subsubsection{Generator Model}

The equivalent electric circuit of the DFIG, in synchronously rotating $d-q$ reference frame can be presented by the following stator and rotor voltage equations [14]

$$
\left\{\begin{array}{l}
v_{d s}=-R_{s} i_{d s}-\omega_{s} \lambda_{q s}+\frac{d \lambda_{d s}}{d t} \\
v_{q s}=-R_{s} i_{q s}-\omega_{s} \lambda_{d s}+\frac{d \lambda_{q s}}{d t} \\
v_{d r}=-R_{r} i_{d r}-\left(\omega_{s}-\omega_{r}\right) \lambda_{q r}+\frac{d \lambda_{d r}}{d t} \\
v_{d r}=-R_{r} i_{q r}-\left(\omega_{s}-\omega_{r}\right) \lambda_{d r}+\frac{d \lambda_{q r}}{d t}
\end{array}\right.
$$


where $\lambda_{s}$ and $\lambda_{r}$ are the stator and rotor flux linkages; $v_{s}$ and $v_{r}$ are the stator and rotor voltages; $i_{s}$ and $i_{r}$ are the stator and rotor currents; $R_{s}$ and $R_{r}$ are the stator and rotor resistance; $\omega_{s}$ is the generator stator speed. The flux linkage in (4.4) is defined as

$$
\left\{\begin{array}{l}
\lambda_{d s}=-L_{s} i_{d s}+L_{m} i_{d r} \\
\lambda_{q s}=-L_{s} i_{q s}+L_{m} i_{q r} \\
\lambda_{d r}=-L_{r} i_{d r}+L_{m} i_{d s} \\
\lambda_{q r}=-L_{r} i_{q r}+L_{m} i_{q s}
\end{array}\right.
$$

where $L_{s}, L_{r}$, and $L_{m}$ are the stator, rotor, and magnetizing inductances, respectively.

As the stator of a WT is directly connected to the grid, $v_{s}$ is fixed by the grid voltage. Thus, the rotor voltage $v_{r}$ must be precisely determined by a converter and applied to design the DFIG control. From equations (4.4) and (4.5), the voltages of the rotor can be written as

$$
\begin{gathered}
v_{d r}=R_{r} i_{d r}+\left(L_{r}-\frac{L_{m}^{2}}{L_{s}}\right) \frac{d i_{d r}}{d t}-s \omega_{s} \sigma L_{r} i_{q r} \\
v_{q r}=R_{r} i_{q r}+\left(L_{r}-\frac{L_{m}^{2}}{L_{s}}\right) \frac{d i_{q r}}{d t}-s \omega_{s} \sigma L_{r} i_{d r}+s\left(\frac{L_{m} V_{s}}{L_{s}}\right)
\end{gathered}
$$

where $\sigma$ is the leakage coefficient $\left(\sigma=1-L_{m}^{2} /\left(L_{s} L_{r}\right), s\right.$ is the generator $\operatorname{slip}(s=$ $\left.\omega r / \omega_{s}, \omega_{r}=\omega_{s}-\omega_{t}\right)$, and $v_{s}$ is the stator voltage.

\subsection{Description of the Control Systems}

Detail of the proposed control scheme for combinatorial STATCOM and DFIG-based WT is illustrated in Figure 4.2. As it can be seen, it is schematically divided into three main blocks. Control of the DFIG is achieved by control of the RSC and control of the GSC [13-15]. The vector control strategy is used for the active and reactive power control of WT with DFIG system. 


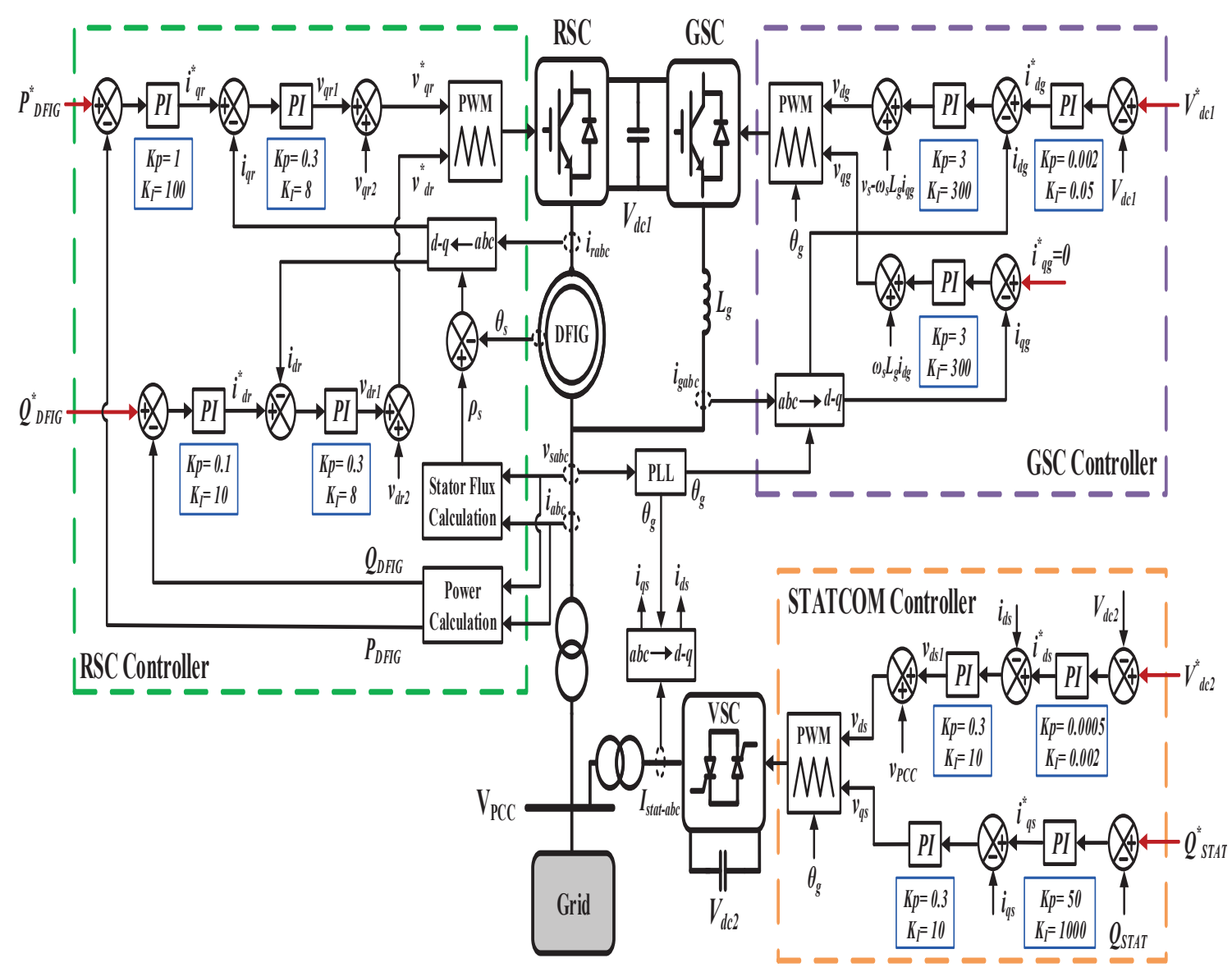

Figure 4.2: Overall control scheme of the proposed system. $v_{d r 2}=-s \omega_{s} \sigma L_{r} i_{q r} ; v_{q r 2}=$ $-s \omega_{s} \sigma L_{r} i_{d r}+s v_{s} L_{m} / L_{s}$.

\subsubsection{Rotor side Converter (RSC) Controller}

The RSC provides independent control of DFIG active and reactive power, $P_{D F I G}$ and $Q_{R S C}$. During normal operation, the RSC is considered to be reactive neutral by setting $Q_{R S C}^{*}=0$. The reactive power control can be applied during the fault to keep the stator voltage $v_{s}$ within the desired level based on regional grid codes, when the DFIG feeds into a week grid. In order to achieve independent control of them, the three-phase rotor currents $i_{r a b c}$ are transformed into $d-q$ components $i_{q r}$ and $i_{d r}$ in the stator flux oriented reference frame. The active power output $P_{D F I G}$, measured at the grid terminals of the DFIG is compared with the reference active power extracted from the WT characteristic 
(see Figure 4.1) and then error is given as the input to a PI controller to generate the reference q-axis rotor current $i_{q r}^{*}$. The error between $i_{q r}^{*}$ and the actual q-axis current $i_{q r}$ is used as inputs to the linear PI controllers. In order to achieve good transient responses, the output $v_{q r 1}$ is decoupled by $v_{q r 2}=-s \omega_{s} \sigma L_{r} i_{d r}+s v_{s} L_{m} / L_{s}$ to produce the reference q-axis voltage $v_{r q}^{*}$.

Moreover, the reference DFIG reactive power $Q_{R S C}^{*}$ is compared with its actual measurement at the grid side to generate the error signal, which is passed through a PI controller to provide the reference d-axis signal $i_{d r}^{*}$. Then, it is compared with its actual signal $i_{d r}$ to produce an error, which is then used to provide the required d-axis voltage signal $v_{d r 1}$ using a PI controller after decoupling by $v_{d r 2}=-s \omega_{s} \sigma L_{r} i_{q r}$. These are then used by the PWM module to generate the IGBT gate control signals to drive the rotor-side IGBT converter. The maximum value of the reference rotor current $i_{r}^{*}$ is limited to 1 p.u. When $i_{d r}^{*}$ and $i_{q r}^{*}$ are such that the magnitude is higher than 1 p.u the $i_{q r}^{*}$ component is reduced in order to bring back the magnitude to 1 p.u.

\subsubsection{Grid side Converter (GSC) Controller}

The objective of the GSC is to maintain the dc-link voltage constant. In this effort, the GSC control scheme is also designed to set the reactive current component at $i_{q g}^{*}=0$. This setting is reasonable since the DFIG converters are rated for only $30 \%$ of the generator rating, which are primarily used for transferring the active power from the rotor to the grid.

The actual signal of the dc-link voltage $V_{d c 1}$ is compared with its command value $V_{d c 1}^{*}$ to form the error signals, which is used as input to the PI controller to generate the reference d-axis current $i_{d g}^{*}$. The three-phase currents of the GSC are transformed to $d-q$ components $i_{q g}$ and $i_{d g}$ by applying the synchronously rotating reference frame transformation. Then, the error between the reference signals $\left(i_{q g}^{*}\right.$ and $\left.i_{d g}^{*}\right)$ and the actual 
$d-q$ axis current $\left(i_{q g}\right.$ and $\left.i_{q g}\right)$ are used as inputs to the linear PI controllers. The obtained voltage signals $v_{d g 1}$ and $v_{q g 1}$ are decoupled by $v_{s}-\omega_{s} L_{g} i_{q g}$ and $\omega_{s} L_{g} i_{d g}$, respectively, to produce the reference $d-q$ axis voltage $v_{d g}^{*}$ and $v_{q g}^{*}$. They are then used by the PWM module to generate the IGBT gate control signals to drive the rotor-side IGBT converter.

\subsubsection{STATCOM Controller}

In this effort, a STATCOM is used to provide the desired amount of reactive power during the fault to help the fault ride-through capability of the DFIG. Its control operation is somewhat similar to the GSC: The actual signal of the dc-link voltage $V_{d c 2}$ and the reactive power $Q_{S T A T}$ are compared with their reference values $\left(V_{d c 2}^{*}\right.$ and $\left.Q_{S T A T}^{*}\right)$ and then error signals are passed through the PI controllers to engender the reference $d-q$ axis currents $i_{d s}^{*}$ and $i_{d s}^{*}$, respectively. The errors between these reference values and the corresponding actual $d-q$ current components of the STATCOM are passed through the PI controllers. The voltage signals $v_{d s 1}$ and $v_{q s 1}$ are compensated by the corresponding cross coupling terms to obtain the $d-q$ voltage signals $v_{d s}^{*}$ and $v_{q s}^{*}$. They are then used by the PWM module to generate the IGBT gate control signals to drive the rotor-side IGBT converter.

\subsection{Coordinated Reactive Power Control}

In this section, a multi-objective optimization problem (MOP) model, a stochastic normalized simulated annealing (NSA) algorithm, and a fuzzy logic controller (FLC) are used to design an external interface controller for the coordinated reactive power control between the DFIG and the STATCOM, as shown in Figure 4.3. A DFIG-based WT technology is used in this study, because the majority of WTs in power systems are equipped with DFIGs, and a STATCOM is also employed due to its superior dynamic characteristics. However, the proposed coordinated control method is applicable for other similar devices, such as a permanent magnet synchronous generator (PMSG)-based WT and static VAR compensator (SVC). The MOP model is first formulated in order to simultaneously 


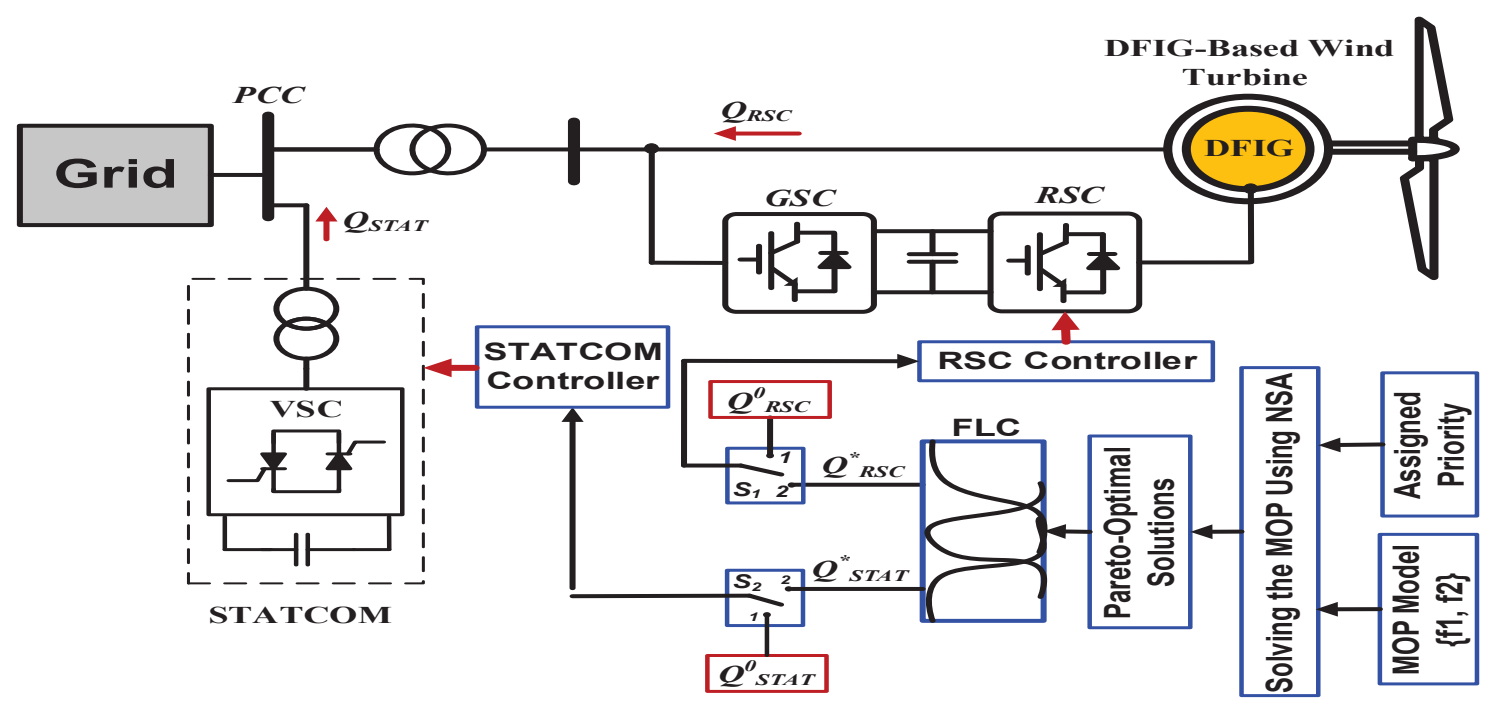

Figure 4.3: Overall block diagram of the proposed PCRPC for online application.

minimize two conflicting objectives: 1) voltage deviations at the point of common coupling (PCC) during and even after the grid faults and 2) low-frequency oscillations of the active power after clearing the faults. In this study, the control variables of the proposed MOP are the reactive power references of $Q_{R S C}^{*}$ and $Q_{S T A T}^{*}$ for both RSC of the DFIG and STATCOM controllers, respectively. The proposed MOP model is then solved by the NSA algorithm in order to find the Pareto-optimal solutions, based on the assigned priorities (weights) for each objective. Each of the Pareto-optimal solutions is used for offline tuning the fuzzy model and fuzzy rules of the FLC. The reason for selecting a NSA-based approach for tuning the FLC is that the derivation of rules from the simple fuzzy reasoning is totally complicated due to the complexity and nonlinearity of the power network. For online applications, the main use of the FLC is that it can quickly change the $Q_{R S C}^{*}$ and $Q_{S T A T}^{*}$ during grid faults, it is possible to reduce the level of voltage reductions at the PCC, and to rapidly dampen the low-frequency oscillations of the active power in the system.

The overall block diagram of the proposed control approach is shown in Figure 4.3. In this study, no effort will made to optimize the steady-state control system, as the goal of the effort emphasizes only the encounter of the faults. Thus, switches $S_{1}$ and $S_{2}$ are 
kept in position 1 in the steady-state condition with fixed reactive power commands of the $Q_{R S C}^{0}$ and $Q_{S T A T}^{0}$. Once the fault occurs, $S_{1}$ and $S_{2}$ switch to position 2 and the FLC, as an external online controller, provides the coordinated reactive power control for the STATCOM and the RSC.

\subsubsection{MOP Model Formulation}

In this section, the proposed coordinated reactive power control is formulated as a multiobjective programming model with the following objectives and constraints.

\section{Objectives}

When a fault occurs at some points of the grid, the WT experiences a voltage sag situation at the PCC and may disconnect from the grid due to a severe voltage stability issue. This problem can be solved by supporting the reactive power during the fault in order to decrease the level of voltage reductions, and thereby improve the fault ride-through capability of the WT. In this effort, the voltage severity index (VSI) is defined to quantify the deviation of the voltage at the PCC, given by

$$
\mathrm{VSI}=\frac{\sum_{t=T_{s}}^{T} \Delta V_{P C C}^{t}}{T-T_{s}}
$$

where $T$ is the considered transient time frame, $T_{s}$ is the fault starting time, and $\triangle V_{P C C}$ is the voltage deviation, calculated by

$$
\Delta V_{P C C}^{t}=\left\{\begin{array}{l}
\frac{\left|V_{P C C}^{t}-V_{P C C}^{0}\right|}{V_{P C C}^{0}}, \quad \text { if } \frac{\left|V_{P C C}^{t}-V_{P C C}^{0}\right|}{V_{P C C}^{0}} \geq \alpha \\
0, \quad \text { otherwise }
\end{array}\right.
$$

where $V_{P C C}^{0}$ is voltage magnitude at the PCC before the fault occurrence, $V_{P C C}^{t}$ is the voltage magnitude of the PCC at time $t$ obtained from time-domain simulation, and $\alpha$ is 
the threshold to define unacceptable voltage deviation level, which can be set according to the international grid codes, e.g., 10\% for Danish grid codes [3].

Also, the sudden lack of the active power generation, due to providing the reactive power with the RSC of the DFIG, might cause a power imbalance during the fault. This will excite the low-frequency oscillations on the generator rotor angle, resulting in fluctuations of the active power. This study proposes a transient power severity index (TPSI) to quantify the transient power performance of the system buses following the clearance of the disturbance

$$
\mathrm{TPSI}=\frac{\sum_{i=1}^{N} \sum_{t=T_{c}}^{T}\left(\left|P_{i}^{t}-P_{i}^{0}\right| / P_{i}^{0}\right)}{N \times\left(T-T_{c}\right)}
$$

where $N$ is the total number of buses in the system, $T_{c}$ is the fault clearing time, $P_{i}^{0}$ is power magnitude of bus $i$ before the fault occurrence, and $P_{i}^{t}$ is the power magnitude of bus $i$ at time $t$ obtained from time-domain simulation.

Here, the first objective is minimization of the VSI, $f_{1}$, and second objective is minimization of the TPSI, $f_{2}$, calculated by (2.8) and (2.10), respectively. It should be noted that (4.8) and (4.10) cannot be explicitly calculated and they need a time-domain simulation to achieve the post-disturbance voltage and power trajectories.

\section{Constraints}

In this study, the constraints of the MOP model are represented as the steady-state constraints and dynamic constraints. The steady-state constraints consist of power flow balance and steady-state operational limits for pre-contingency state

$$
\left\{\begin{array}{l}
P_{G}-P_{L}-P(V, \theta)=0 \\
Q_{G}-Q_{L}-Q(V, \theta)=0
\end{array}\right.
$$




$$
\left\{\begin{array}{l}
S(V, \theta) \leq S^{\max } \\
V^{\min } \leq V \leq V^{\max } \\
P_{G}^{\min } \leq P_{G} \leq P_{G}^{\max } \\
Q_{G}^{\min } \leq Q_{G} \leq Q_{G}^{\max } \\
Q_{S T A T}^{\min } \leq Q_{S T A T} \leq Q_{S T A T}^{\max }
\end{array}\right.
$$

where (4.11) represents the active and reactive power balance for each bus, and $P_{G}$ and $Q_{G}$ represent the active and reactive power generations, $P_{L}$ and $Q_{G}$ represent the active and reactive power loads, $P$ and $Q$ represent the power flow equations, $V$ is the voltage bus, and $\theta$ is the voltage angle. (4.12) represents the operational limits on line flow, steady-state voltage magnitude, generators output capacity (including conventional generators and DFIG), and the STATCOM reactive power capacity.

Also, adding dynamic constraints such as the rotor angle stability constraint is important for the proposed optimization model to avoid the voltage instability. Generally, the rotor angle stability is tested by examining the rotor angle deviation against a certain threshold [166]. In this study, the following constraint is included to ensure the rotor angle stability for any contingency

$$
\left[\max \left(\Delta \delta_{i j}^{T}\right)\right] \leq \beta
$$

where $\left[\max \left(\Delta \delta_{i j}^{T}\right)\right]$ represents the the maximum rotor angle deviation between any two generators during the transient period $T$ and $\beta$ is the threshold which can be set to $\pi$ for the extreme case [166]. 


\subsubsection{Solution Method}

\section{Pareto Optimality}

The two-objective optimization problem presented in Section III-A requires to be solved. Based on the priority of the objective functions, a MOP has multiple solutions, which can form a Pareto-optimal set (set of all the Pareto optimal solutions) [166], where decisionmaker may select any of them depending on practical needs. Given the control variables vector $\boldsymbol{X}=\left[x_{1}, x_{2}, \ldots x_{n}\right]$, a vector $\boldsymbol{X}^{*}=\left[\begin{array}{llll}x_{1}^{*} & x_{2}^{*} \ldots & x_{n}^{*}\end{array}\right]$ is the Pareto-optimal set, or the non-dominated solutions, if any point in $\boldsymbol{X}$ satisfies $f_{j}(\boldsymbol{X}) \leq f_{j}\left(\boldsymbol{X}^{*}\right)$ for all $j=1,2, \ldots, m\left(f_{j}\right.$ denotes to objective function, while $m$ is the number of the objective function).

\section{Normalized Simulated Annealing (NSA)}

When dealing with a MOP, stochastic methods are superior to classical strategies because they can simultaneously find Pareto-optimal solutions [54]. The stochastic NSA algorith$\mathrm{m}$ has the ability to search in very large spaces of candidate solutions and avoid becoming trapped in local minima, with lower computational time compared to other stochastic methods. This technique was early introduced by Kirkpatrick et al. for finding a global minimum of an optimization function [55]. It is based on an analogy between the way in which a metal cools and freezes into a minimum energy crystalline structure (annealing process). The algorithm employs a random search, where random moves are accepted with a probability function $p r=\exp (-\Delta f / T f)$, where $\Delta f$ is increasing in $f$ and $T f$ is the system temperature during the cooling process [21].

Since two objective functions presented in Section III-A must be prioritized according to the power system operator's needs, a cooling schedule, taking into account the prioritization of the these objective functions, is proposed by assigning a different initial temperature for each objective, i.e., $T f_{1}$ and $T f_{2}$. If the initial temperature is given to an objective in a high order (i.e. high priority), the probability of accepting a solution is 
higher than that objective with lower initial temperature (i.e. less given priority). Moreover, since these two objective functions are not coherent, these should be normalized by dividing their initial values in order to make them comparable to each other. The rest of the optimization parameters are the number of iterations, maxiter, upper and lower bounds of the variables, $\boldsymbol{X}^{\max }$ and $\boldsymbol{X}^{\text {min }}$, which determine the search area and the rate of the temperatures change $r_{b}$, which is positive and less than 1 . The detailed steps of the NSA algorithm are shown in Table 4.1.

\subsubsection{Tuning the FLC Model Using NSA}

The FLC used in this effort is based for two inputs; i.e., the two initial temperatures $T f_{1}$ and $T f_{2}$ assigned to two objectives $f_{1}$ and $f_{2}$, respectively. The output of the FLC system are defined with two optimal control variables, i.e. $Q_{R S C}^{*}$ and $Q_{S T A T}^{*}$, which are obtained by the NSA optimization algorithm in form of Pareto-optimal solutions. Each of the two input and two output variables is divided into the three fuzzy subsets corresponding to meanings of "low" $(L)$, "medium" $(M)$, and "high" $(H)$. All fuzzy subsets are modeled with sigmoid membership functions [167]. Fuzzy input subsets and fuzzy output subsets are generated by selecting the parameters $m$ and $\sigma$, as given in Table 4.2 and shown in Figure 4.4.

In this study, the changes made to parameter of $m$ are only taken into consideration for tuning the output fuzzy subsets model. As shown in Table 4.3, the continuous variables $y_{1}$ and $y_{2}$ indicate adjustable parameters the output fuzzy subsets. The effects of making changes on output fuzzy membership functions have been simultaneously studied by using the NSA algorithm. This optimization approach is presented for tuning the fuzzy model (Figures 4.4(b) and (c)) by determining the suitable value of $y_{1}$ and $y_{2}$ and also creating the fuzzy rules (or Pareto-optimal classification rules) using the obtained Pareto-optimal solutions. The reason for selecting the NSA-based approach for tuning 
Table 4.1: Normalized Simulated Annealing (NSA) Algorithm

1 Define an initial $T f_{j}$ for each objective and Set $i t e r=1$

2 Create randomly an initial solution $\boldsymbol{X}=\left[x_{1}, x_{2}, \ldots x_{n}\right]$ for $\boldsymbol{X}^{\min } \leq \boldsymbol{X} \leq \boldsymbol{X}^{\max }$

3 Calculate objective values of $\left\{f_{1}(\boldsymbol{X}), f_{2}(\boldsymbol{X}), \ldots, f_{m}(\boldsymbol{X})\right\}$

4 Produce new random solutions using the nonuniform mutation operator

for $i=1$ to $n$

Create a uniformly distributed random number $0<\boldsymbol{r a n d}_{i}<1$

Generate a random binary number bin

$$
\begin{aligned}
& \text { if bin }=1 \text { then } \\
& x_{i}^{\text {new }}=x_{i}^{\text {iter }}+\left(x_{i}^{\text {max }}-x_{i}^{\text {iter }}\right) \cdot \operatorname{rand}_{i} \cdot \exp (- \text { iter } / \max i t e r) \\
& \text { else } \\
& x_{i}^{\text {new }}=x_{i}^{\text {iter }}+\left(x_{i}^{\text {iter }}-x_{i}^{\text {min }}\right) \cdot \text { rand }_{i} \cdot \exp (- \text { iter } / \max i t e r)
\end{aligned}
$$

end if

end for

5 Normalized the objective functions for obtained solutions

$$
\begin{gathered}
f_{j}^{\text {norm }}(\boldsymbol{X})=f_{j}\left(\boldsymbol{X}^{\text {new }}\right) / f_{j}\left(\boldsymbol{X}^{\text {iter }}\right) \\
\text { for } j=1,2, \ldots, m
\end{gathered}
$$

6 Calculate the probabilities of the new solutions

$$
\begin{gathered}
\Delta f_{j}=f_{j}^{\text {norm }}\left(x_{1}^{\text {new }}, \ldots, x_{n}^{\text {new }}\right)-f_{j}^{\text {norm }}\left(x_{1}^{\text {iter }}, \ldots, x_{n}^{\text {iter }}\right) \\
\quad \text { for } j=1,2, \ldots, m \\
\text { if } \Delta f_{j} \geq 0 \text { then } \\
p r_{j}=\exp \left(-\Delta f_{j} / T f_{j}\right) \text { else } p r_{j}=1 \\
\text { end if }
\end{gathered}
$$

7 Produce $m$ random numbers rand $_{j}$

if $\forall \operatorname{rand}_{j}<p r_{j}$ for $j=1,2, \ldots, m$ then

$$
x_{i}^{i t e r+1}=x_{i}^{\text {new }} \text { else } x_{i}^{\text {iter }+1}=x_{i}^{\text {iter }}
$$

end if

8 Set $T f_{j}=r_{b} T f_{j}$ and iter $=$ iter +1

if iter $<\max i t e r$ then goto step 3

else $\boldsymbol{X}^{*}=\boldsymbol{X}^{\text {maxiter }}$

The Pareto-optimal solutions are $\boldsymbol{X}^{*}=\left[x_{1}^{*}, x_{2}^{*}, \ldots, x_{n}^{*}\right]$ end if

Exit

the FLC is that the proposed combinatory DFIG-based WT and the STATCOM is a highdimensional multivariate time-varying system and the derivation of rules from the simple 


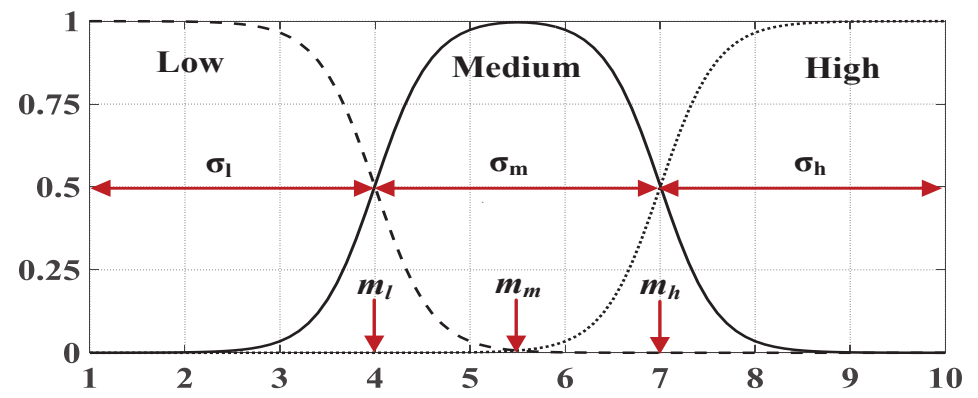

(a)

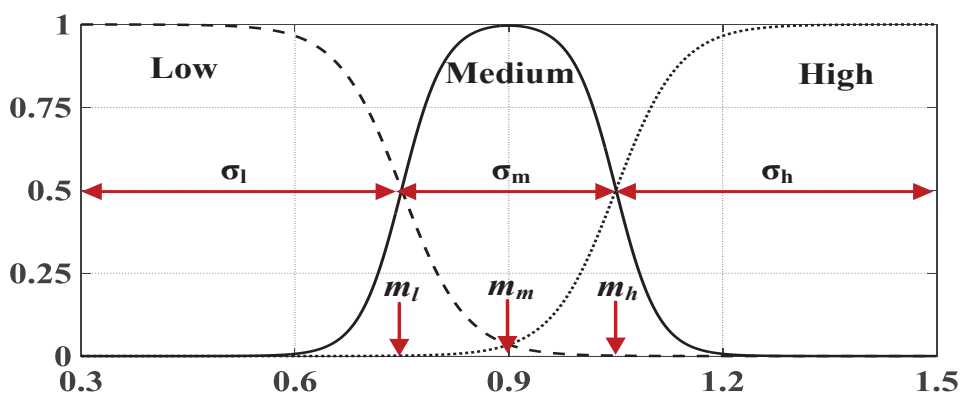

(b)

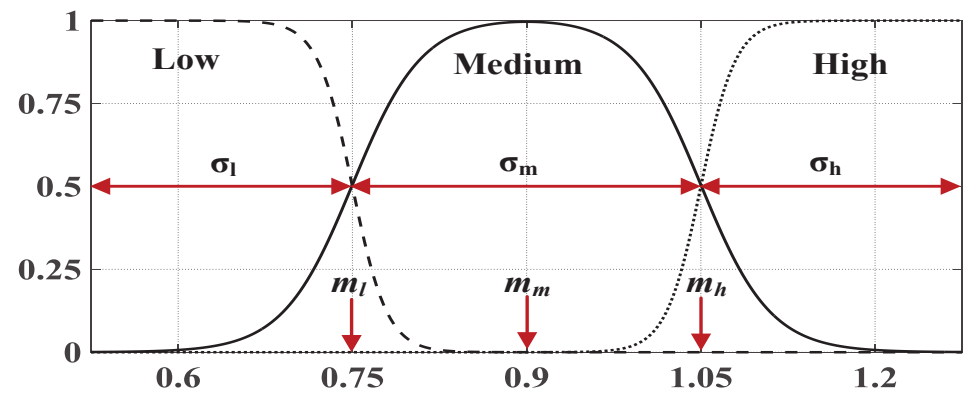

(c)

Figure 4.4: Fuzzy membership functions. (a) Fuzzy input subsets of the $T f_{1}$ and $T f_{2}$. (b) Fuzzy output subset of the $Q_{R S C}^{*}$. (c) Fuzzy output subset of the $Q_{S T A T}^{*}$.

fuzzy reasoning is entirely complicated. The procedure of fuzzy model and fuzzy rules tuning is designed using the following five steps:

\section{Step 1) Initialization}

There are two control variables $\left(Q_{R S C}\right.$ and $\left.Q_{S T A T}\right)$ for the NSA algorithm and also two adjustable parameters $\left(y_{1}\right.$ and $\left.y_{2}\right)$ for the FLC model, which can be formulated as the vector $\boldsymbol{X}=\left[\begin{array}{ll}Q_{R S C} & Q_{S T A T}\end{array}\right]^{T}$ and the vector $\boldsymbol{Y}=\left[\begin{array}{ll}y_{1} & y_{2}\end{array}\right]^{T}$. The upper and lower bounds, 
Table 4.2: Parameters Used to Generate Initial Sigmoid Membership Functions for Input and Output Variables

\begin{tabular}{lccccccc}
\hline \hline Variables & Variation & \multicolumn{2}{c}{ Low subset } & \multicolumn{2}{c}{ Mid subset } & \multicolumn{3}{c}{ High subset } \\
& range & $m_{l}$ & $\sigma_{l}$ & $m_{m}$ & $\sigma_{m}$ & $m_{h}$ & $\sigma_{h}$ \\
\hline$T f_{1,2,3}$ & {$[1,2, \ldots, 10]$} & 4 & 3 & 5.5 & 3 & 7 & 3 \\
$Q_{R S C}^{*}$ & $(0.31 .5)$ & 0.75 & 0.45 & 0.9 & 0.3 & 1.05 & 0.45 \\
$Q_{S T A T}^{*}$ & $(0.51 .275)$ & 0.75 & 0.225 & 0.9 & 0.3 & 1.05 & 0.225 \\
\hline \hline
\end{tabular}

Table 4.3: Parameters Used to Generate Tunable Sigmoid Membership Functions for Output Variables

\begin{tabular}{lcccccc}
\hline \hline Variables & \multicolumn{2}{c}{ Low subset } & \multicolumn{2}{c}{ Mid subset } & \multicolumn{2}{c}{ High subset } \\
& $m_{l}$ & $\sigma_{l}$ & $m_{m}$ & $\sigma_{m}$ & $m_{h}$ & $\sigma_{h}$ \\
\hline$Q_{R S C}^{*}$ & $y_{1}$ & 0.45 & $y_{1}+0.15$ & 0.3 & $y_{1}+0.3$ & 0.45 \\
$Q_{S T A T}^{*}$ & $y_{2}$ & 0.225 & $y_{2}+0.15$ & 0.3 & $y_{2}+0.3$ & 0.225 \\
\hline \hline
\end{tabular}

$\boldsymbol{X}^{\max }$ and $\boldsymbol{X}^{\min }$ of the control parameters and the upper and lower bounds, $\boldsymbol{Y}^{\max }$ and $\boldsymbol{Y}^{\text {min }}$ of the fuzzy variables must be specified to determine the range of the searching apace, given by

$$
\left\{\begin{array}{l}
0.3 \leq Q_{R S C} \leq 1.5 \text { MVar } \\
0.5 \leq Q_{S T A T} \leq 1.27 \text { MVar } \\
0.69 \leq y_{1} \leq 0.81,0.69 \leq y_{2} \leq 0.81,
\end{array}\right.
$$

In this work, the limitations of the control variables for the optimization problem are determined with two principles; 1) RSC of the DFIG should have the minimum contribution of 20\% (0.3 MVar from 1.5 MVar) for fault ride-through requirement during the fault; and 2) STATCOM must provide the minimum value of steady-state reactive power $0.5 \mathrm{M}$ Var and the maximum $85 \%$ of its total capacity (i.e., 1.27 MVar from 1.5 MVar) for safe operation during the fault. Hence, the upper and lower bounds for optimization process are: $\boldsymbol{X}^{\min }=\left[\begin{array}{ll}0.3 & 0.5\end{array}\right]^{T}, \boldsymbol{X}^{\max }=\left[\begin{array}{ll}1.5 & 1.27\end{array}\right]^{T}, \boldsymbol{Y}^{\min }=\left[\begin{array}{lll}0.69 & 0.69\end{array}\right]^{T}$, and $\boldsymbol{Y}^{\max }=\left[\begin{array}{ll}0.81 & 0.81\end{array}\right]^{T}$. 
As an arbitrary choice, each objective of the MOP model $f_{1}\left(Q_{R S C}, Q_{S T A T}\right)$ and $f_{2}\left(Q_{R S C}, Q_{S T A T}\right)$, where $f_{1}$ and $f_{2}$ denote to VSI and TPSI, respectively, is assigned an initial temperature of $T f_{1}$ and $T f_{2}$ based on their priority selection, i.e., low $\left(T f_{1,2}=1\right)$, medium $\left(T f_{1,2}=5\right)$, and high $\left(T f_{1,2}=10\right)$. As mentioned before, two initial temperatures $T f_{1}$ and $T f_{2}$ are considered for the input fuzzy system, which are divided into the three fuzzy subsets. For three input fuzzy subsets, there are 7 possible cases that are needed to convert into equivalent fuzzy rules after 7 optimization round, as shown in Table 4.4.

\section{Step 2) Selecting initial temperature}

At the first round of the optimization, the initial temperatures of $T f_{1}=10$ and $T f_{2}=10$ for two objectives is selected with the corresponding initial fuzzy input High - High as the rule number 1, while it is shifted to the next rule at the end of each optimization round, until reaching the rule number 7 (i.e., updating the fuzzy rules). Moreover, the output fuzzy subsets (Fig. 4.4(b) and (c)) are generated using initial values of $y_{1}=y_{2}=0.75$ at the first round of the optimization, and are then regenerated simultaneously at the next round of the optimization based on the updated variables of $y_{1}$ and $y_{2}$ (i.e., updating the fuzzy model). In the proposed NSA algorithm, 100 iterations (maxiter $=100)$ is considered for each round of the optimization. Set iter $=1$ and start the optimization process with step 3.

\section{Step 3) Generating new random solution}

Create randomly an initial solution $\boldsymbol{X}^{i t e r}=\left[\begin{array}{ll}Q_{R S C}^{i t e r} & Q_{S T A T}^{i t e r}\end{array}\right]^{T}$ inside the pre-defined searching space. To produce new control variables $\boldsymbol{X}^{n e w}=\left[Q_{R S C}^{n e w} Q_{S T A T}^{n e w}\right]^{T}$ based on the current solutions, an update for each parameter is calculated at each iteration. If random binary $\operatorname{bin}=1$, then

$$
\boldsymbol{X}^{\text {new }}=\boldsymbol{X}^{i t e r}+\left(\boldsymbol{X}^{\max }-\boldsymbol{X}^{i t e r}\right) \cdot \operatorname{rand}(0,1) \cdot \exp (-i t e r / \max i t e r)
$$

else 
Table 4.4: Fuzzy Rules Generated by NSA

\begin{tabular}{ccccc}
\hline \hline Rule No. & & $\begin{array}{c}\text { Fuzzy Input } \\
T f_{1}-T f_{2}\end{array}$ & \multicolumn{1}{c}{ Fuzzy Output } \\
& & $Q_{R S C}^{*}-Q_{S T A T}^{*}$ \\
\hline \hline 1 & IF & High - High & THEN & Medi-High \\
2 & IF & High - Medi & THEN & Medi - High \\
3 & IF & High - Low & THEN & High - High \\
4 & IF & Medi-High & THEN & Low - High \\
5 & IF & Medi-Low & THEN & High - High \\
6 & IF & Low - High & THEN & Low - Low \\
7 & IF & Low - Medi & THEN & Low - Low \\
\hline \hline
\end{tabular}

$$
\boldsymbol{X}^{n e w}=\boldsymbol{X}^{i t e r}+\left(\boldsymbol{X}^{i t e r}-\boldsymbol{X}^{\text {min }}\right) \cdot \operatorname{rand}(0,1) \cdot \exp (-i t e r / \max i t e r)
$$

Also, new adjustable parameters $\boldsymbol{Y}^{\text {new }}=\left[y_{1}^{\text {new }}, y_{2}^{\text {new }}\right]$ are computed based on a random value between $[-0.5,0.5]$ and the current temperature of each objective by

$$
\boldsymbol{Y}^{\text {new }}=\boldsymbol{Y}^{\text {iter }}+\operatorname{rand}(-0.5,0.5) \cdot\left[\left(T f_{1}^{\text {iter }}+T f_{2}^{\text {iter }}\right) /\left(T f_{1}^{\text {initial }}+T f_{2}^{\text {initial }}\right)\right]
$$

As the temperature decreases, changes in parameters of $y_{1}$ and $y_{2}$ decreases proportional to the temperature decrease.

\section{Step 4) Selecting new solution for next iteration}

The probability of selecting the new control variables $\boldsymbol{X}^{\text {new }}$ for $f_{1}$ and $f_{2}$ are obtained after normalizing the objective functions, as follows

$$
\begin{gathered}
\left\{\begin{array}{l}
\Delta f_{1}=f_{1}^{n o r m}\left(Q_{R S C}^{n e w}, Q_{S T A T}^{n e w}\right)-f_{1}^{n o r m}\left(Q_{R S C}^{i t e r} Q_{S T A T}^{\text {iter }}\right) \\
\Delta f_{2}=f_{2}^{n o r m}\left(Q_{R S C}^{n e w}, Q_{S T A T}^{n e w}\right)-f_{2}^{n o r m}\left(Q_{R S C}^{i t e r} Q_{S T A T}^{i t e r}\right)
\end{array}\right. \\
p r_{j}= \begin{cases}\exp \left(-\Delta f_{j} / T f_{j}^{i t e r}\right), & \text { if } \Delta f_{j} \geq 0 \\
1, & \text { if } \Delta f_{j}<0\end{cases}
\end{gathered}
$$


for $j=1$ and $j=2$. If $\left(p r_{1}>r_{1}\right)$ and $\left(p r_{2}>r_{2}\right)$

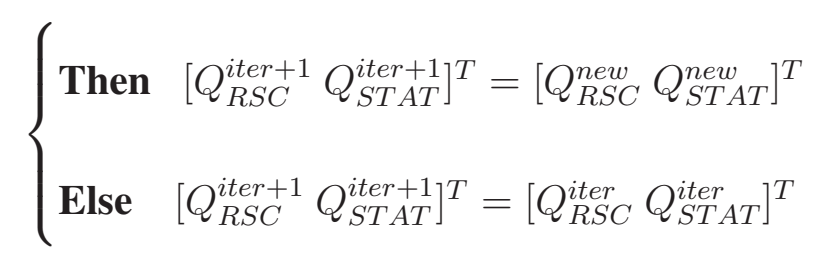

where $r_{1}$ and $r_{2}$ are uniformly distributed random numbers in $[0,1]$. The adjustable parameters of $\boldsymbol{Y}$ for the next iteration of the optimization can be achieved as

$$
\begin{aligned}
& \text { If } Q_{R S C}^{i t e r+1} \in[0.45,0.9] \text { and } Q_{S T A T}^{i t e r+1} \in[0.6,0.9] \text {, }
\end{aligned}
$$

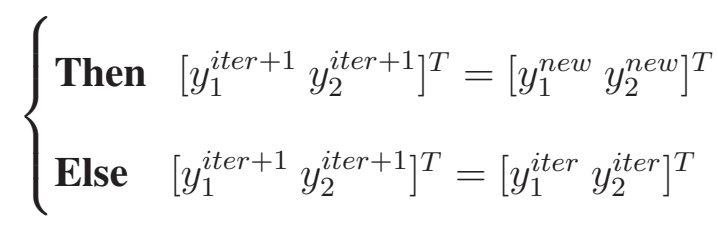

\section{Step 5) Updating fuzzy model and fuzzy rules}

In this step, the Pareto-optimal solutions for control variables $\boldsymbol{X}$ and optimal adjustable parameters $\boldsymbol{Y}$ will be updated after each round (there is 7 round optimization).

Set $T f_{1}^{i t e r+1}=r_{b} T f_{1}^{i t e r}, T f_{2}^{i t e r+1}=r_{b} T f_{2}^{i t e r}$, and iter $=i t e r+1$. If iter $<\max i t e r$, then go to step 3 to keep iterating. Otherwise, the search process will be terminated with $\left[Q_{R S C}^{*} Q_{S T A T}^{*}\right]^{T}=\left[\begin{array}{ll}Q_{R S C}^{\max i t e r} & Q_{S T A T}^{\max i t e r}\end{array}\right]^{T}$ and $\left[y_{1} y_{2}\right]^{T}=\left[\begin{array}{ll}y_{1}^{\max i t e r} & y_{2}^{\max i t e r}\end{array}\right]^{T}$.

The Pareto-optimal solutions $X^{*}$ obtained at each round of optimization process is used to update fuzzy rules, as presented in Table 4.4. Also, the obtained $Y$ values is used to update the output fuzzy subset configurations, as shown in Figures 4.4(b), (c), based on the tunable fuzzy model given in Table 4.2. If the round number of the optimization process is 7 , then stop optimization process(tuning the fuzzy model and fuzzy rules are completed); otherwise, go to step 2. 


\subsection{Computer Simulation Study}

In previous sections, the mathematical formulations are derived in order to find the optimal values of the compensating reactive power for both the RSC of the DFIG and the STATCOM controllers. It was achieved via the FLC, which is properly tuned using the NSA algorithm. In this section, the simulations in MATLAB/SIMULINK are presented to validate the effectiveness of the proposed control approach.

\subsubsection{Power System Case Study}

The original four-machine 12-bus benchmark power system in [168] was used as a platform system to study the flexible ac transmission system (FACTS) device applications for the transient stability analysis. In this study, the single-line diagram of the four-machine 12-bus power system is modified by placing a WT and a STATCOM at bus 6 (PCC), as shown in Figure 4.5. The system covers three geographical areas; area 1 represents the main generation area with two hydro power generation $\mathrm{G} 1$ and G2; area 2 represents the main load center with a hydro power generation G3; and area 3 represents the proposed combination of the 1.5 MW DFIG-based WT and the 1.5 MVar STATCOM. For dynamic studies, in this work, the conventional generators of G1, G2, and G3 are modeled in detail, in which the exciter and turbine governor dynamic models are taken into account. The detailed dynamic specifications of the conventional generators, as well as 12-bus system parameters are given in [168]. Table 4.5 shows the 1.5 MW DFIG parameters, which are used in the simulation results.

A three-phase symmetrical grid fault is considered, since the fault ride-through capability of the regional grid codes mostly refer to this type of fault. Thus, a three-phase fault is applied to the bus 1 end of line $1-6$ at $t=40 \mathrm{~s}\left(T_{s}=40\right)$ and is cleared after $150 \mathrm{~ms}$ $\left(T_{c}=40.15\right)$, resulting in a $40 \%$ depth of voltage dip at the PCC. The offline tuning of 


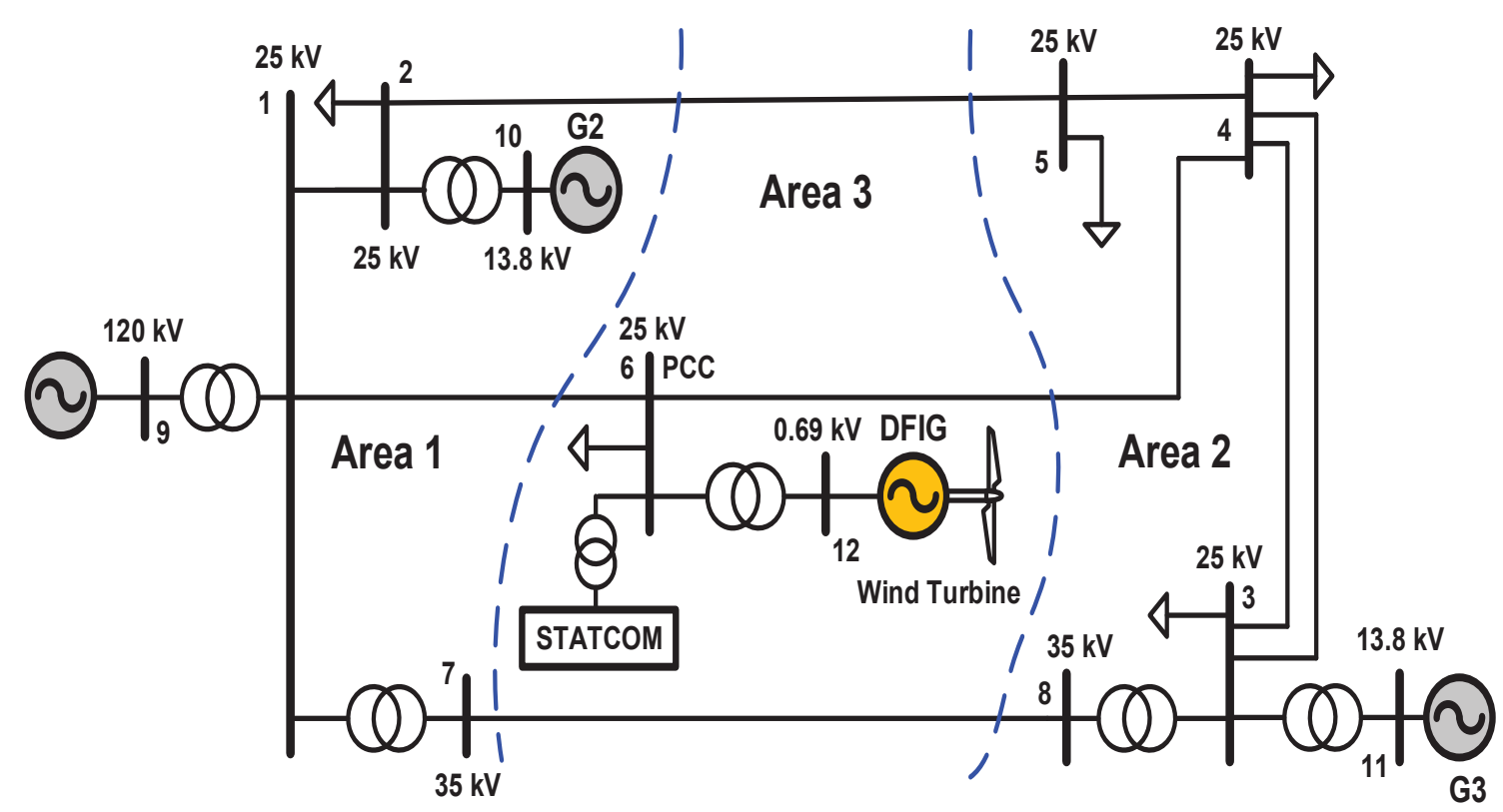

Figure 4.5: Single-line diagram of the multi-machine benchmark power system including a wind turbine and a STATCOM connected to the PCC.

Table 4.5: Parameters of the 1.5-MW DFIG

\begin{tabular}{llll}
\hline \hline Parameters & Value & Parameters & Value \\
\hline \hline Rated power & $1.5 \mathrm{MW}$ & Stator inductance & $0.171 \mathrm{pu}$ \\
Rated voltage & $0.69 \mathrm{kV}$ & Rotor inductance & $0.156 \mathrm{pu}$ \\
Rated frequency & $60 \mathrm{~Hz}$ & Magnetizing inductance & $2.9 \mathrm{pu}$ \\
Rated wind speed & $12 \mathrm{~m} / \mathrm{s}$ & Rotational inertia & $5.04 \mathrm{~s}$ \\
Stator resistance & $0.007 \mathrm{pu}$ & dc-link voltage & $1.2 \mathrm{kV}$ \\
Rotor resistance & $0.005 \mathrm{pu}$ & dc bus capacitor & $50 \mathrm{mF}$ \\
\hline \hline
\end{tabular}

the FLC model, via proposed NSA algorithm, were accomplished by means of MATLAB programming.

\subsubsection{Validation of the NSA Algorithm}

Table 4.6 shows 7 optimization round process results of the tuning algorithm due to the temperature changes of the two objectives based on priority selection (i.e., low (1), medium (5), and high (10)). The corresponding results are used to plot the variation of the ob- 
Table 4.6: Pareto Optimality Results Using NSA Algorithm.

\begin{tabular}{llcccc}
\hline $\begin{array}{l}\text { Case } \\
\text { No. }\end{array}$ & $\begin{array}{c}\text { Priority } \\
T f_{1}-T f_{2}\end{array}$ & $\begin{array}{c}Q_{R S C}^{*} \\
\text { (MVar) }\end{array}$ & $\begin{array}{c}Q_{S T A T}^{*} \\
\text { (MVar) }\end{array}$ & VSI & $T P S I$ \\
\hline \hline 1 & $10-10$ & 1.02 & 1.06 & 0.236 & 0.336 \\
2 & $10-5$ & 1.04 & 1.12 & 0.233 & 0.396 \\
3 & $10-1$ & 1.42 & 1.15 & 0.227 & 0.486 \\
4 & $5-10$ & 0.62 & 1.18 & 0.258 & 0.186 \\
5 & $5-1$ & 1.13 & 1.08 & 0.230 & 0.408 \\
6 & $1-10$ & 0.43 & 0.76 & 0.375 & 0.144 \\
7 & $1-5$ & 0.56 & 0.86 & 0.298 & 0.168 \\
\hline \hline
\end{tabular}
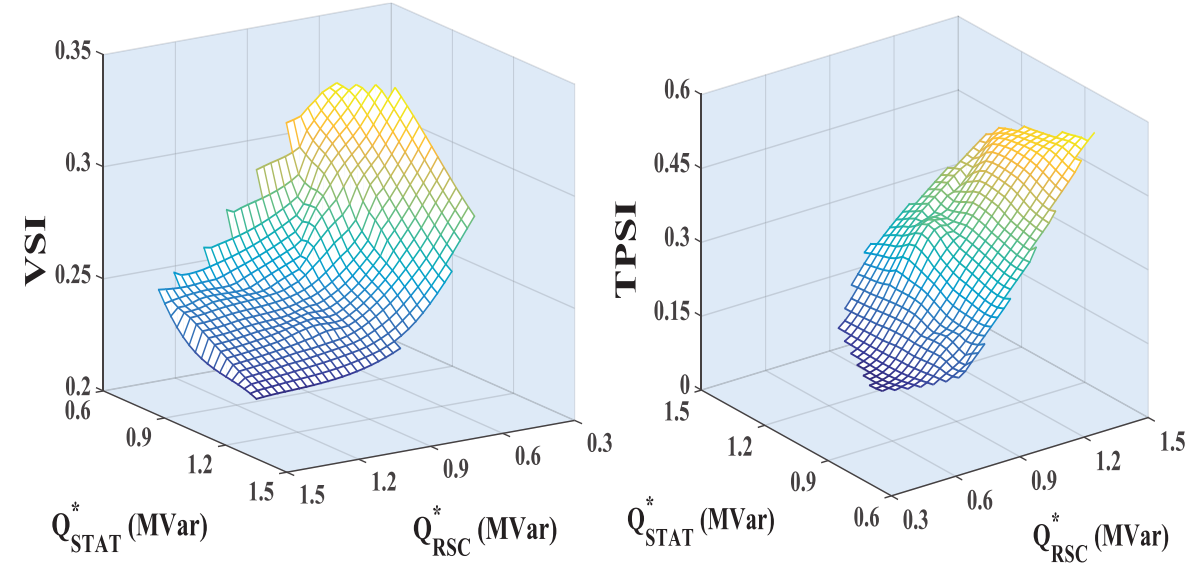

Figure 4.6: Three dimensional representations of control variables versus objective function.

jective functions, $V S I$ and TPSI versus the control variables $Q_{R S C}^{*}$ and $Q_{S T A T}^{*}$, shown in Figure 4.6, which are interpolated for better visualization. It can be seen that increasing the amount of $Q_{D F I G}^{*}$ and $Q_{S T A T}^{*}$ is needed to minimize $V S I$. However, the large value $Q_{R S C}^{*}$ increases the TPSI.

Figure 4.7 schematically depicts the progression of the control variables and objective functions of the optimization problem after 100 iterations, considering the first objective $(V S I)$ as a high priority and the second objective (TPSI) as a low priority. The results clearly show that the reactive power commands and objective functions rapidly converge 


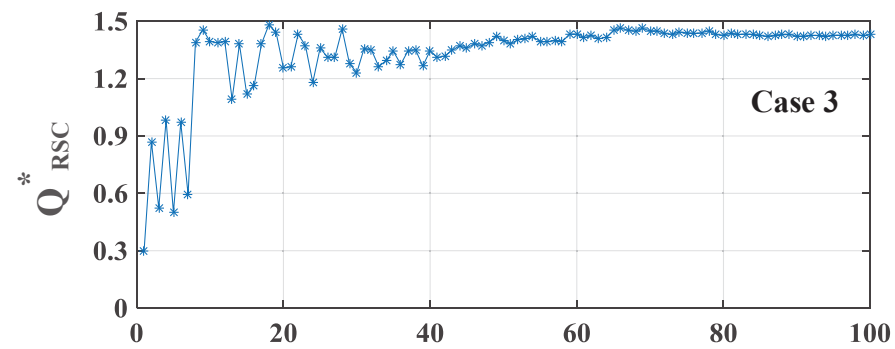

(a)

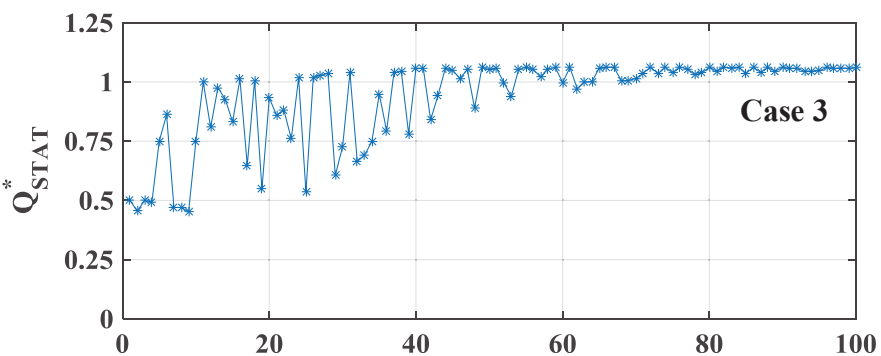

(b)

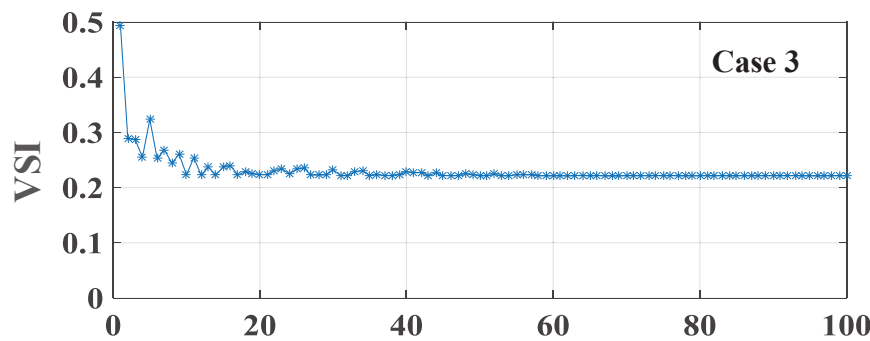

(c)

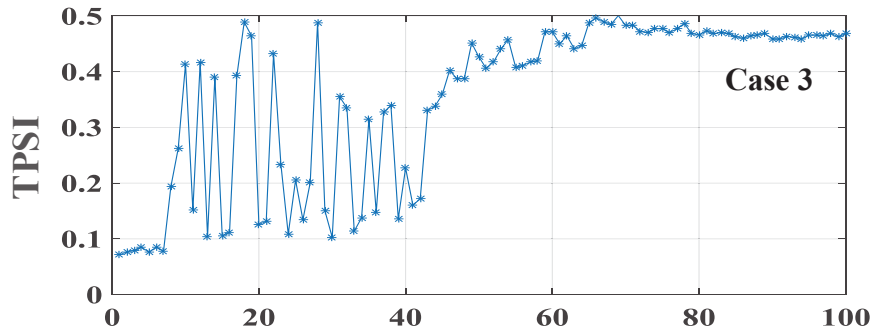

(d)

Figure 4.7: NSA-based optimization progress of the reactive power commands (control variables) and two objectives for the case 3; x-axis denotes to the number of iteration.

in an optimal point during the cooling (annealing) process.

For comparative purposes, the optimization process is verified by another algorithm, i.e., the non-dominant sorting GA version II (NSGA-II) [43]. The NSGA-II approximates 
Table 4.7: Comparison of the NSA Performance with NSGA-II

\begin{tabular}{cccccc}
\hline \hline $\begin{array}{c}\text { Optimization } \\
\text { Algorithm }\end{array}$ & $\begin{array}{c}\text { Number of } \\
\text { iterations(s) }\end{array}$ & $\begin{array}{c}\text { iteration } \\
\text { time(s) }\end{array}$ & $\begin{array}{c}\text { Total } \\
\text { time(s) }\end{array}$ & $\begin{array}{c}\text { Average } \\
\text { error }\end{array}$ & $\begin{array}{c}\text { Error } \\
\text { Variance }\end{array}$ \\
\hline \hline NSA & 100 & 0.41 & 41 & 0.605 & 0.144 \\
NSGA-II & 100 & 1.55 & 155 & 0.121 & 0.017 \\
\hline \hline
\end{tabular}

the entire Pareto front, and no modification could be made to locate only the optimal solutions corresponding to the multi-objectives problems. The best parameters of NSGAII optimization for the proposed problem are achieved with a population size of 60 , a crossover probability of 0.85 , and a mutation probability of 0.15 , resulting with the final Pareto-optimal solutions after 100 iterations. Table 4.7 presents the total computational times, using a PC with an Intel core i7-4770/3.5-GHz, the average errors, and the error variances that were produced by the two methods.

Compared to the NSGA-II algorithm, the proposed method exhibits considerably lower computational times, which is reliable for online application, where the control system needs to act in a very short period of time. The Figure 4.8 shows the Pareto front (set of all the Pareto optimal objective values), which is produced by the tradeoff among the 7 sets of mutually contradicting control variables. These results are compared with 11 optimal objective values obtained by the NSGA-II algorithm.

All these solutions lying on the Pareto front are non-dominated solutions, so that they can be selected with the individual decision makers based on his/her own preference. For some regional grid codes, such as Australia, Denmark and Germany, which supporting the reactive power under fault conditions has highest priority [3], the cases of 2, 3 and 5 in Table 4.6 can be suitable choices. On the contrary, for some regional grid codes, such as Ireland, Spain and the UK, where supplying the active power to the grid during and even after the faults has higher priority compared with reactive power [3], more contribution 


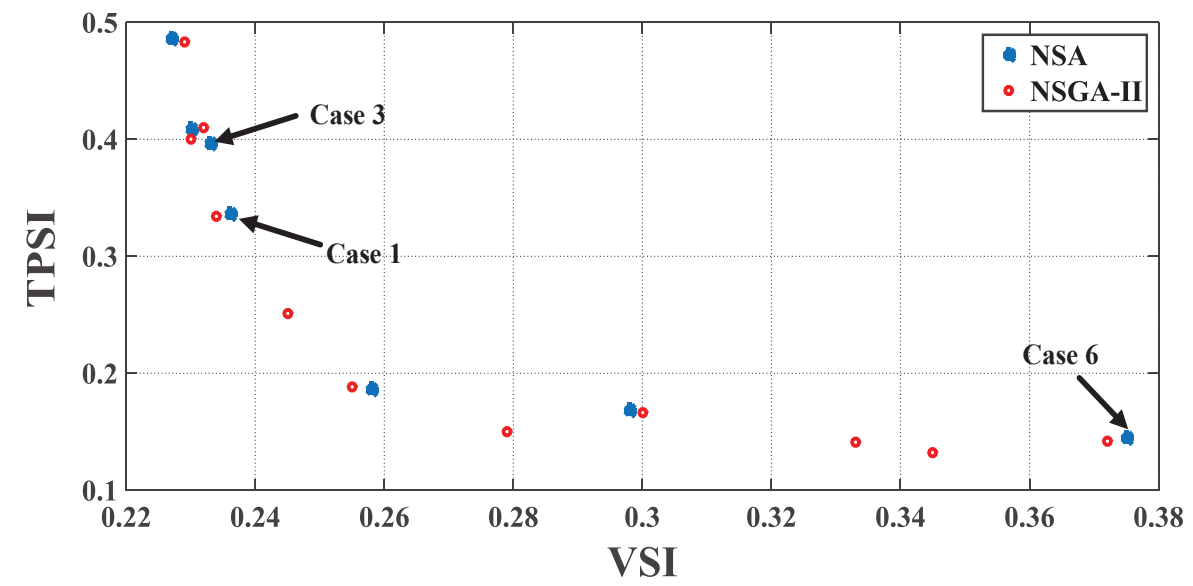

Figure 4.8: Pareto front that was obtained with NSGA-II and NSA algorithm.

of the STATCOM should be taken into consideration in order to compensate required reactive power for the stability improvement. Thus, in these grid reginal grid codes, the cases the cases of 4, 6 and 7 can be proper candidates. The case 1 could be a proper selection, when supplying the active and reactive power have the same importance.

It should be also mentioned that the concept of the proposed reactive control strategy during the fault is applicable to various sizes of the WT and STATCOM. The only variation would be in the upper and lower bounds, $\mathbf{X}^{\max }$ and $\mathbf{X}^{\min }$ of the control variables in order to determine the searching space for the proposed optimization problem.

\subsubsection{Simulation Case Studies}

For validation purposes, the dynamic performance of the combinatorial WT and the STATCOM, were reinforced with the proposed control approach in case numbers of 1, 3, and 6; compared with the case without the proposed control system, where all control parameters under fault condition were the same with the steady-state condition.

Case No.1 from Table 4.6: in case number 1, the proposed control system was optimally designed to minimize both objective functions by assigning the highest priority $\left(T f_{1}=10\right.$ and $\left.T f_{2}=10\right)$ to the $V S I$ and $T P S I$. In this case, both the RSC and the 


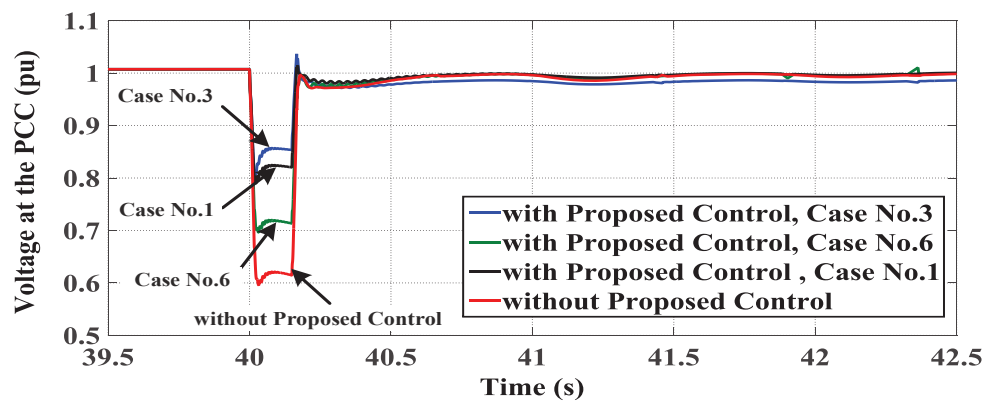

(a)

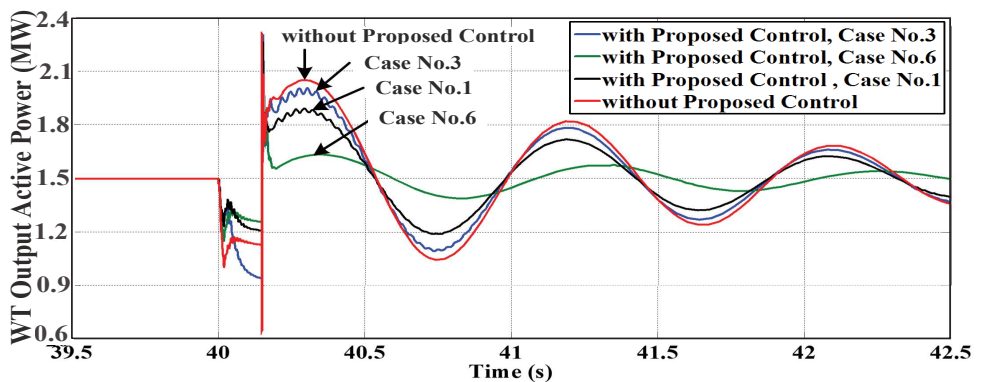

(b)

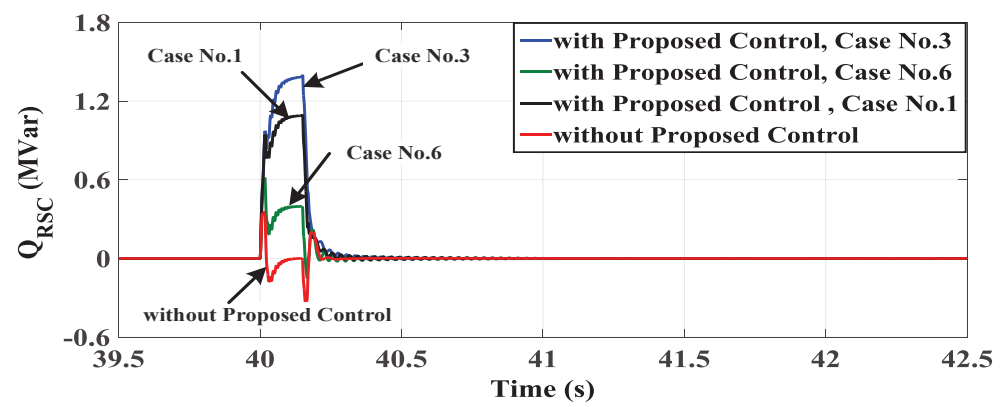

(c)

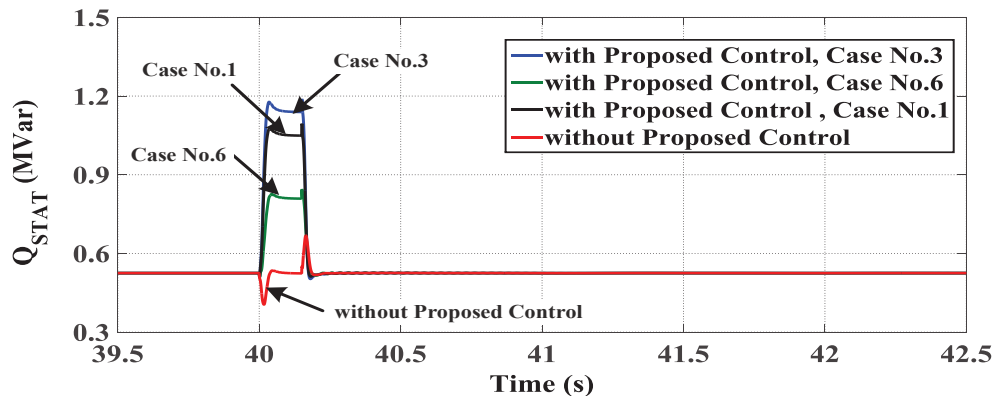

(d)

Figure 4.9: Dynamic response of the 12-bus system with and without proposed control system considering case 1, case 3, and case 6. (a) Voltage at PCC. (b) Output active power at the PCC. (c) Output reactive power of WT. (d) Output reactive power of STATCOM. 
STATCOM have almost a same contribution for generating the reactive powers during the faults in order to improve transient behavior of the voltage and power at the PCC during and after clearing the fault. Accordingly, reactive power commands of the RSC and the STATCOM were quickly changed to high values of $Q_{R S C}^{*}=1.02 \mathrm{MVar}$ and $Q_{S T A T}^{*}=1.06$ MVar during the fault, as shown in Figures 4.9(c) and (d). In this case, the depth of the voltage dip is about 24\%, as shown in Figure 4.9(a). The post-fault active power oscillations in case number 1 are damped more rapidly than in case number 3 , but this is still less rapidly than in case number 3 (see Figure 4.9(b)). These results confirm that the proposed control system enhances the LVRT capability of the WT and power oscillation damping of the system during transient conditions.

Case No.3 from Table 4.6: in case number 3, the proposed control system was optimally designed to minimize the voltage deviations at the PCC during the fault by assigning the highest priority to the objective $V S I$. Thus, more contribution of the RSC and STATCOM should be taken into consideration, in order to compensate required reactive power for voltage stability improvement of the weak power system. As shown in Figure 4.9(a), the proposed control approach significantly reduces the magnitude of voltage sag to around $15 \%$. Accordingly, reactive power commands of the RSC and the STATCOM were quickly changed to high values of $Q_{R S C}^{*}=1.42 \mathrm{MVar}$ and $Q_{S T A T}^{*}=1.15 \mathrm{MVar}$ during the fault, as shown in Figure 4.9(c) and Figure 4.9(d). After the fault clearing at $t=40.15 \mathrm{~s}$, reactive power values return to their predefined values in the steady-state condition. As it can be noticed in Figure 4.9(b), the proposed control system in case 3 is not able to reduce the active power oscillations at the PCC due to lower priority of TPSI compared to $V S I$.

Case No.6 from Table 4.6: in case number 6, the proposed control system was optimally designed to enhance power oscillation damping in power system after clearing the fault, by assigning the highest priority to the objective TPSI. In Figure 4.9(a), it can be 
observed that this case is unable to improve the voltage deviation at the PCC due to lower priority $V S I$ compared to $T P S I$, where magnitude of voltage sag reaches approximately $30 \%$. Accordingly, the reactive power commands of the RSC and the STATCOM were both set at low values of $Q_{R S C}^{*}=0.43 \mathrm{MVar}$ and $Q_{S T A T}^{*}=0.76 \mathrm{MVar}$ during the fault, as shown in Figure 4.9(c) and Figure 4.9(d). The post-fault active power oscillations in case 3 is damped more rapidly than other cases (Figure 4.9(b)).

\subsection{Summary}

Since, many WTs have been electrically integrated into a weak power grid with low shortcircuit ratios and significant voltage dips, the DFIG converters are not able to provide adequate reactive power and voltage support, due to their limited capacity. Thus, the application of huge STATCOMs have been increasingly proposed to help with the uninterrupted operation of a wind farm technology equipped with DFIGs under disturbances, and improve the resilience of the power system including the RES systems. The STATCOM can provide rapid and smooth reactive compensation and voltage control under steady-state and transient conditions. Reactive power coordination between the WT and the STATCOM is very important for improving the LVRT capability and thereby increasing the resilient of the overall power system. This chapter has been presented a computational intelligence technique in order to design an external interface controller for the coordinated reactive power control between the WT and the STATCOM, during the faults. The overall problem is formulated as a multiobjective optimization model, minimizing two conflicting objectives, and solved by stochastic NSA algorithm. This approach provides a Pareto front for decision makers to determine the optimal tradeoff between reducing the voltage deviations during and after the fault and damping the active power oscillations during the recovery period. Compared to the NSGA-II method, it is clearly confirmed the NSA exhibits considerably lower computational times (NSA is around 3.7 times faster than 
NSGA-II). The transient active power performance of the system after the fault clearing time is quantified by the so-called TPSI. For online applications, the optimal commanded values of the compensating reactive power for both RSC of the DFIG and the STATCOM controllers have been achieved via an FLC which is properly tuned using the NSA algorithm. Simulation results have shown that the proposed control approach can successfully improve the LVRT capability and can operate as an external damping controller for the WT, and therefore, improve the post-fault power oscillation damping of system. 


\section{CHAPTER 5}

\section{ACTIVE AND REACTIVE POWER CONTROL OF THREE-PHASE SINGLE-STAGE GRID-TIED PV MODULE-INTEGRATED CONVERTER}

\subsection{Overview}

This chapter discusses the application of a three-phase module-integrated converter (MIC) incorporated into a grid-tied photovoltaic (PV) system. A current source inverter (CSI) with dc voltage boost capability, namely the single-stage power conversion system, is proposed for a three-phase PV MIC system. A power control strategy based on synchronous frame proportional-integral (PI) regulators with structural simplicity and fast dynamic response is designed to control the proposed CSI-based MIC system. The principle goal is to provide a very smooth and fast dynamic response to step jumps and falls in the active and reactive power commands. It is demonstrated that the active and reactive powers which are exchanged between the PV MIC and the grid can be obtained through controlling the modulation index and the modulation angle introduced in the phasor PWM (PPWM) switching pattern. In the design of the PI controller, two distinct $d$ - and $q$-axis currents must be extracted in order to be independently regulated. However, due to the poor disturbance rejection capabilities, the $d$ and $q$ axes are not fully decoupled, and the step changes in one axis generate transients in the other, which might lead to power quality and performance deterioration. Thus, a multivariable PI controller with a faster dynamic response and superior axis decoupling capability is proposed, in which the active and reactive powers are almost fully decoupled so that the step changes in one power reference value negligibly affect the other.

Up to the present time, as far as the authors are aware, there have been no reports, that fully consider the active and reactive power control of a CSI-based MIC system by using a multivariable PI-based controller. Thus, this chapter attempts to fill this void by developing the multivariable PI current regulator for the three-phase single-stage grid-tied CSI 
topology. First, a switching pattern based on the phasor pulse-width-modulated (PPWM) technique is employed to produce the switching signals through introducing the modulation index and the modulation angle, namely control parameters. Then, the state-space averaging method, in combination with the $d q$ reference frame, is used to derive the largeand small-signal models in order to study the dynamic behaviors of the CSI-based MIC system. The control design procedure corresponding to the multivariable PI regulator is presented, in which the active and reactive powers are almost fully decoupled so that the step changes in one power reference value negligibly affect the other. Finally, the performance of the multivariable PI control method has been simulated in MATLAB/Simulink software and then experimentally verified through a 300-VA laboratory prototype. The results are compared with that of the conventional PI regulation approach.

\subsection{Configuration and Modulation Strategy}

The system architecture for the PV MIC system based on the three-phase grid-tied CSI topology is shown in Figure 5.1. The CSI circuit consists of a bridge with six reverse blocking MOSFET switches $\left(S_{1}-S_{6}\right)$, each of them is in series with a diode, a dc-link inductor $L_{d c}$ as the main energy storage component with small resistance $R_{d c}$, and a PV source emulator, representing the output voltage of the PV array $\left(v_{p v}\right)$. The system is connected to the three-phase grid voltages $v_{a g}, v_{b g}, v_{c g}$ through the RLC filter unit, which smooths out the pulsed phase currents from the dc link. The voltages $v_{a w}, v_{b w}, v_{c w}$ and currents $i_{a w}, i_{b w}, i_{c w}$ represent the output voltages and currents of the converter, as shown in Figure 5.1.

A CSI is typically operated using either a selected harmonic elimination (SHE) switching pattern or a space vector PWM (SVPWM) switching pattern. However, the boost ratio $\beta$, which can be defined as $V_{m} / V_{p v}$ (where $V_{m}$ and $V_{p v}$ are the magnitude of the rated phase voltage and PV emulator voltage, respectively), is below 1.0 for SHE- and slightly above 


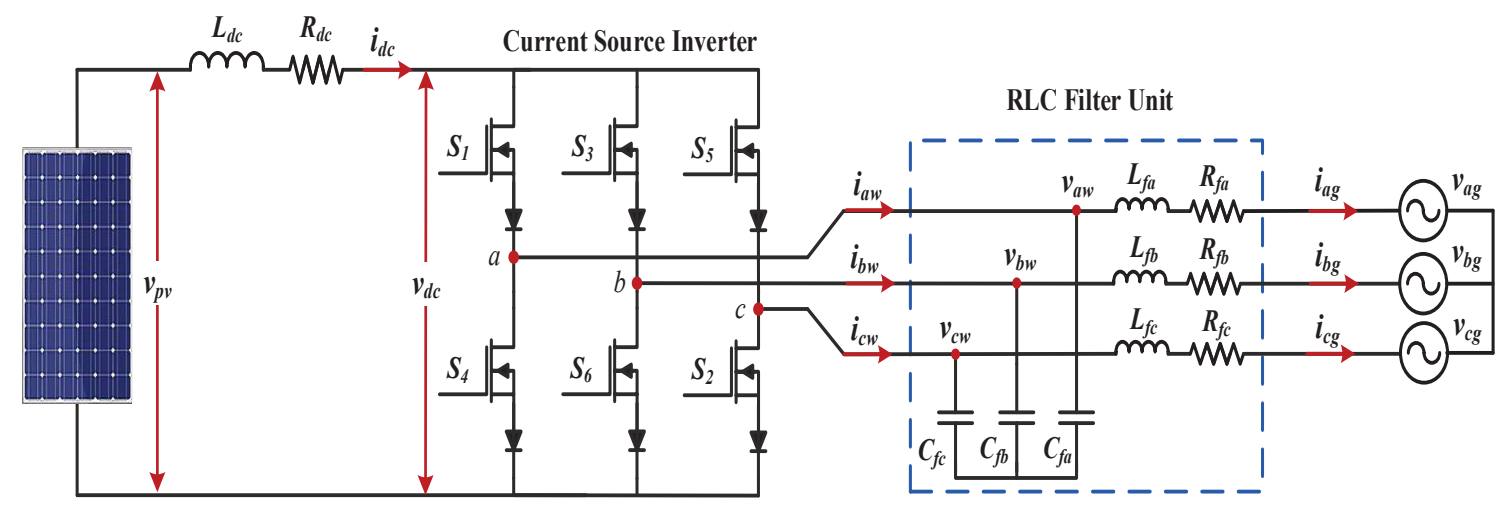

Figure 5.1: Three-phase single-stage grid-tied CSI test system.

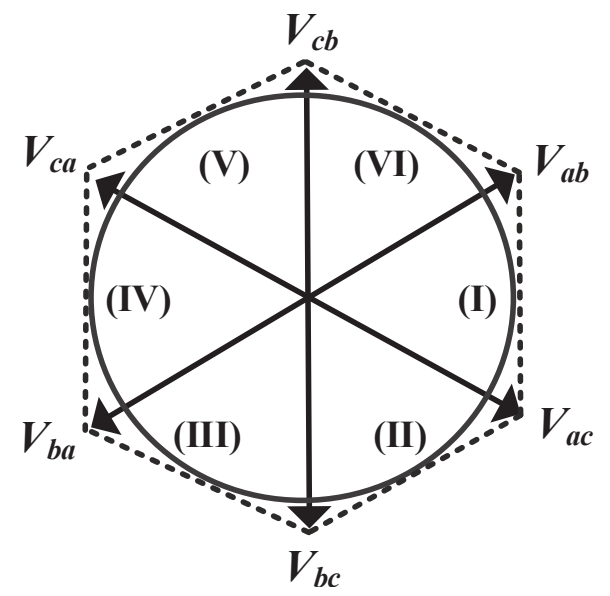

Figure 5.2: Six sections in line-to-line voltage phasors.

1.0 for SVPWM-based CSIs. To remedy this, a phasor PWM (PPWM) switching technique for a CSI topology formulated based on the voltage phasor quantities is proposed in [169]. The boost ratio in the PPWM-based CSI, namely the boost inverter, can easily be higher than 3.00, while the THD remains below 5\%. Based on the PPWM modulation strategy, there are six sectors separated by six line-to-line voltage phasors, $V_{a b}, V_{b c}$, $V_{c a}, V_{b a}, V_{c b}$, and $V_{a c}$, according to the zero-crossing points of grid voltages, as shown in Figure 5.2. The switching sequence of the boost inverter switches for all six sectors is depicted in Figure 5.3, where $V_{1}$ and $V_{2}$ are different line-to-line voltage phasors in each sector. Each of these sector is performed by three operating modes with switching cycle 


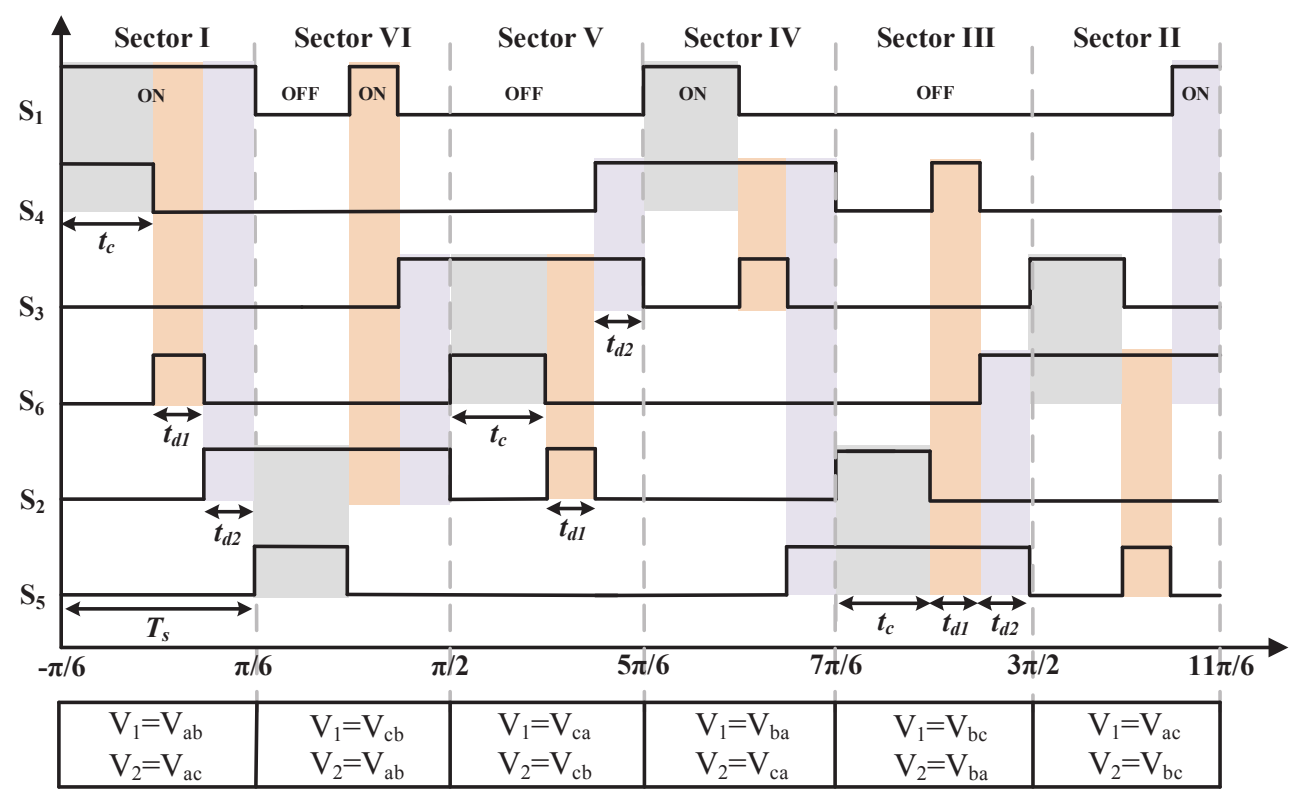

Figure 5.3: Switching sequence of all six sectors.

$T_{s}$, which includes three time intervals: $t_{c}$ is the time interval for charging the dc-link inductor, and $t_{d 1}$ and $t_{d 2}$ are the time intervals for the discharging of the injection of the current to the CSI output terminals, and $T_{s}=t_{c}+t_{d 1}+t_{d 2}$. At any instance, there are only two active conducting switches, one from the upper MOSFETs $\left(S_{1}, S_{3}, S_{5}\right)$ and one from the lower MOSFETs $\left(S_{4}, S_{6}, S_{2}\right)$, to form a flow pass for the inductor current, $i_{d c}$.

For instance, Sector I $\left(-\pi / 6 \leq \alpha_{0}<\pi / 6\right)$ can be performed in three time intervals as shown in Figure 5.4 and described below.

Interval $1\left[0-t_{c}\right]$ :

$S_{1}$ and $S_{4}$ are turned on and others switches are off. The dc-link inductor is being charged to boost the output voltage, while output currents are supplied by $C_{f a}, C_{f b}$, and $C_{f c}$ (see Figure 5.4(a)).

Interval $2\left[t_{c}-\left(t_{c}+t_{d 1}\right)\right]$ :

During this period of time, $S_{1}, S_{6}$ are closed, where the inductor current $i_{d c}$ discharges through $C_{f a}, C_{f b}$, and the grid voltages $v_{a g}, v_{b g}$ (see Figure 5.4(b)). 

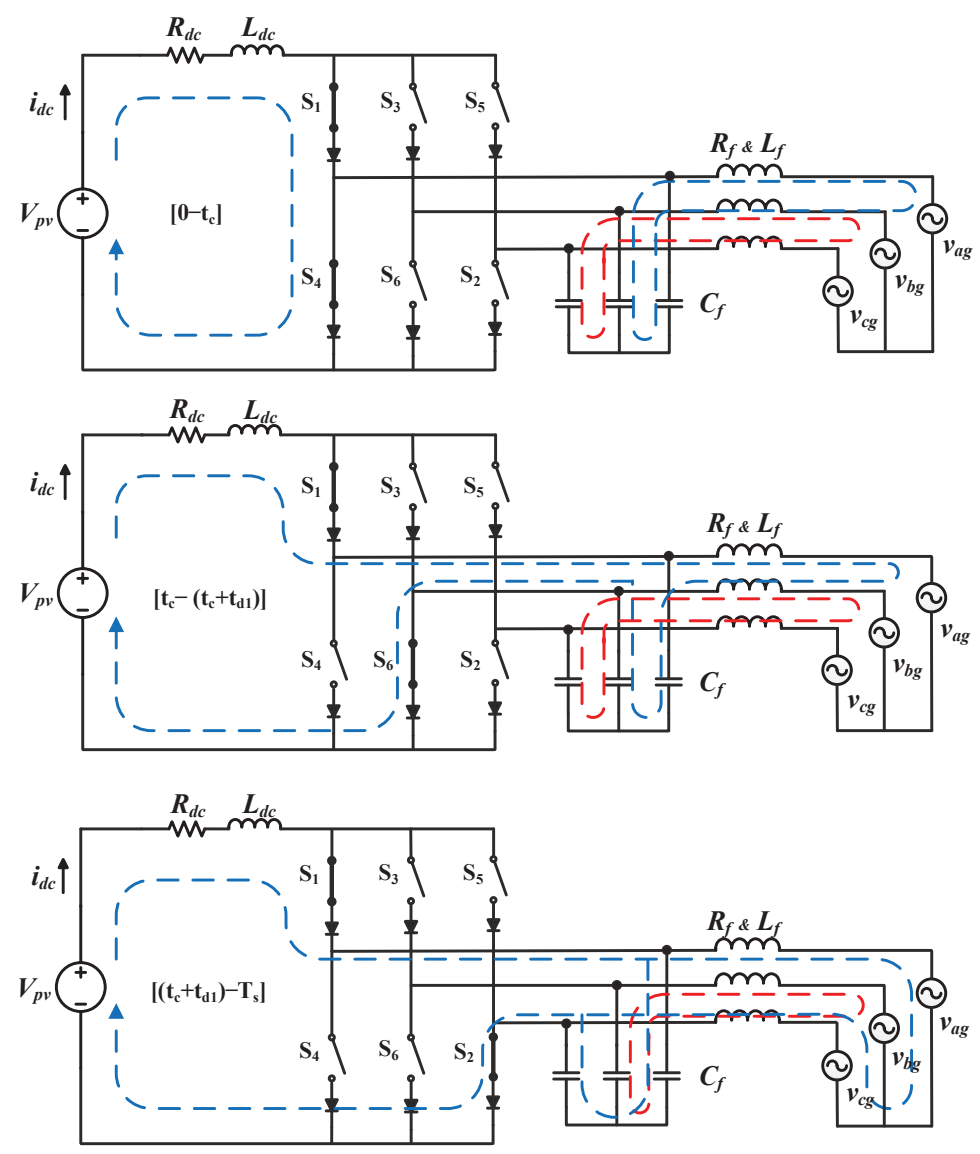

Figure 5.4: Three intervals for proposed switching patterns for Sector I.

Interval $3\left[\left(t_{c}+t_{d 1}\right)-T_{s}\right]$ :

$S_{1}, S_{2}$ are $\mathrm{ON}$ and $i_{d c}$ discharges the through $C_{f a}, C_{f c}$, and $v_{a g}, v_{c g}$, whereas $i_{b g}$ is supplied by $C_{f b}, C_{f c}$ (see Figure 5.4(c)). In principle, one of the switches is $\mathrm{ON}$ for the entire $T_{s}$ duration in a given sector, while other switches operate at the switching frequency.

Figure 5.5 shows the voltage and current waveforms of the dc-link inductor with the assumption that the dc-link resistance $R_{d c}$ can be negligible, and the PV input voltage $v_{p v}$ remains constant over a switching cycle $T_{s}$ (i.e., $v_{p v}=V_{p v}$ ). Referring to this figure, the dynamic equations of the dc-link inductor current over one switching cycle can be obtained as 


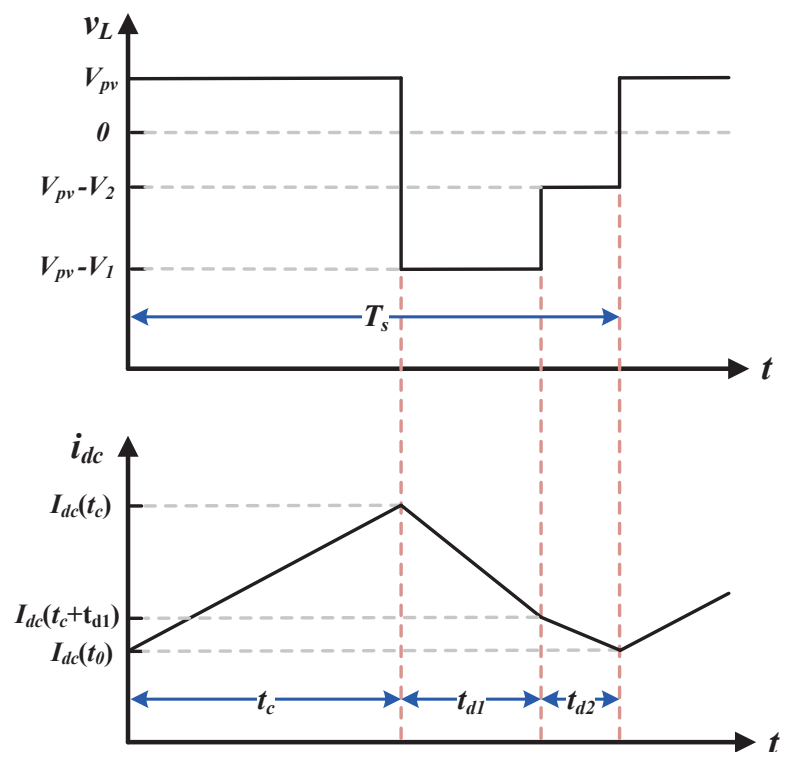

Figure 5.5: Voltage and current of the dc-link inductor over one $T_{s}$ in a CSI, while $V_{1}$ and $V_{2}$ are line-line voltage output.

$$
\begin{cases}I_{d c\left(t_{c}\right)}-I_{d c\left(t_{0}\right)}=\frac{V_{p v}}{L_{d c}} t_{c}, & 0<t \leq t_{c} \\ I_{d c\left(t_{c}+t_{d 1}\right)}-I_{d c\left(t_{c}\right)}=\frac{V_{p v}-V_{1}}{L_{d c}} t_{d 1}, & t_{c}<t \leq t_{c}+t_{d 1} \\ I_{d c\left(T_{s}\right)}-I_{d c\left(t_{c}+t_{d 1}\right)}=\frac{V_{p v}-V_{2}}{L_{d c}} t_{d 2}, & t_{c}+t_{d 1}<t \leq T_{s}\end{cases}
$$

where $I_{d c\left(t_{0}\right)}$ and $I_{d c\left(T_{s}\right)}$ represent dc-link inductor currents at the beginning and the end of one switching cycle, respectively. Summation of the above equations yields the equation below

$$
V_{p v} T_{s}-L_{d c}\left(I_{d c\left(T_{s}\right)}-I_{d c(0)}\right)=V_{1} t_{d 1}+V_{2} t_{d 2}
$$

In the steady-state and balanced conditions (i.e., $\left.I_{d c\left(T_{s}\right)}=I_{d c(0)}\right)$, the equation can be further simplified as

$$
V_{p v} T_{s}=V_{1} t_{d 1}+V_{2} t_{d 2}
$$

If the voltage $V_{a b}=\sqrt{3} V_{m} \cos (\omega t)$ is considered as the reference signal, the charging and discharging intervals for all six sectors are considered as below [170]: 


$$
\left\{\begin{array}{l}
t_{d 1}=m_{i} T_{s} \cos \left(\omega t-\alpha_{0}\right) \\
t_{d 2}=m_{i} T_{s} \cos \left(\omega t-\alpha_{0}-\frac{2 \pi}{3}\right) \\
t_{c}=T_{s}-\left(t_{d 1}+t_{d 2}\right)
\end{array}\right.
$$

where $0<m_{i} \leq 1$ is the modulation index that can be utilized to adjust the level of dc current at the dc-link inductor, and modulation angle $\alpha_{0}$ is the phase shift with respect to the voltage $v_{a b}$ that can be applied to control the power factor of the CSI-based MIC system, and $\omega$ is the grid angular frequency.

The right-hand side of the equation (5.3) for Sector I $\left(-\pi / 6 \leq \alpha_{0}<\pi / 6\right)$ with $V_{1}=V_{a b}$ and $V_{2}=V_{a c}=\sqrt{3} V_{m} \cos (\omega t-\pi / 3)$ can be expressed as

$$
\left\{\begin{array}{l}
V_{1} t_{d 1}=\frac{\sqrt{3} m_{i} T_{s} V_{m}}{2}\left(\cos \left(\alpha_{0}\right)+\cos \left(2 \omega t-\alpha_{0}\right)\right) \\
V_{2} t_{d 2}=\frac{\sqrt{3} m_{i} T_{s} V_{m}}{2}\left(\cos \left(\alpha_{0}+\pi / 3\right)+\cos \left(2 \omega t-\alpha_{0}\right)\right)
\end{array}\right.
$$

Substituting (5.5) into (5.3) yields

$$
V_{p v} T_{s}=\frac{3}{2} m_{i} T_{s} V_{m} \cos \left(\alpha_{0}+\pi / 6\right)
$$

Since the boost ratio $\beta=V_{m} / V_{p v}, m_{i}$ can be obtained from (5.6)

$$
m_{i}=\frac{2}{3 \beta \cos \left(\alpha_{0}+\pi / 6\right)}
$$

The variation of the boost ratio $\beta$, as a function of modulation index $m_{i}$ and modulation angle $\alpha_{0}$, is depicted in Figure 5.6 for Sector I, where $-\pi / 6 \leq \alpha_{0}<\pi / 6$ (see Figure 6.4). The graph shows that the boost ratio of the PPWM-based CSI increases with the reduction of the modulation index. Also, for a constant $m_{i}=0.2$, the boost ratio $\beta$ increases with the increase of the modulation angle.

\subsection{State-Space Average Modeling of Grid-Tied CSI-Based MIC}

In this section, the state-space averaging technique is applied to derive the large- and small-signal models of the three-phase grid-tied CSI-based MIC system. These two mod- 


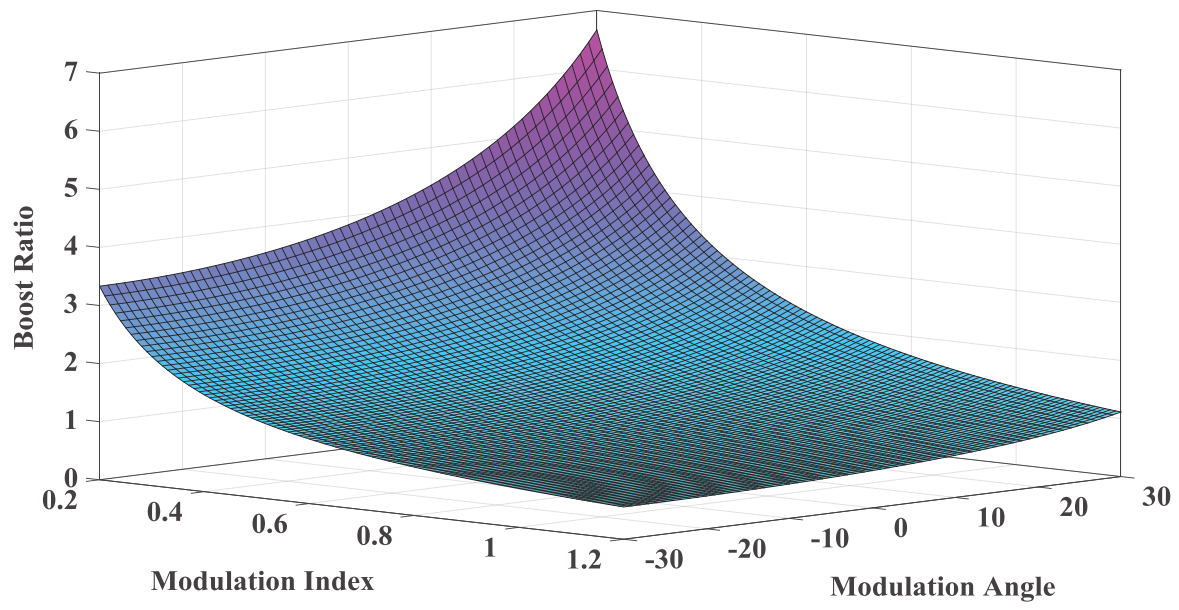

Figure 5.6: Variations of boost ratio $\beta$ versus modulation index $m_{i}$ and the modulation angle $\alpha_{0}$ (degree) for Sector I.

els are established in the following two steps. First, the averaging method is applied to obtain the equivalent circuits of the three-phase grid-tied CSI. These circuits indicate the dynamic behavior of the CSI in both charging and discharging times. A rotating reference frame transformation is used to convert the large-signal model of six distinct sectors (i.e., Sector I to Sector VI in Figure 5.3) into a single state-space representation. Then, the linearization method can be used on the large-signal model to obtain the CSI's small-signal model.

\subsubsection{Large-Signal Model}

The circuit diagrams, corresponding to the three possible switching states of the grid-tied CSI for Sector I, are shown in Figure 5.7. By applying the periodic averaging technique [171], the state-space model can be written as

$$
d x / d t=A_{I} x+B u .
$$

where $x$ is the approximate state vector $x=\left[\begin{array}{lllll}i_{d c} & i_{a g} & i_{b g} & v_{a b}^{w} & v_{b c}^{w}\end{array}\right]^{T}$ and $u$ is the input vector $u=\left[\begin{array}{lll}v_{p v} & v_{a b}^{g} & v_{b c}^{g}\end{array}\right]^{T}$. In this model, the current $i_{c g}$ and voltage $v_{c a}^{w}$ have been written in terms of $i_{a g}, i_{b g}$ and $v_{a b}^{w}, v_{b c}^{w}$, respectively. Thus, the state-space variables remain the same 


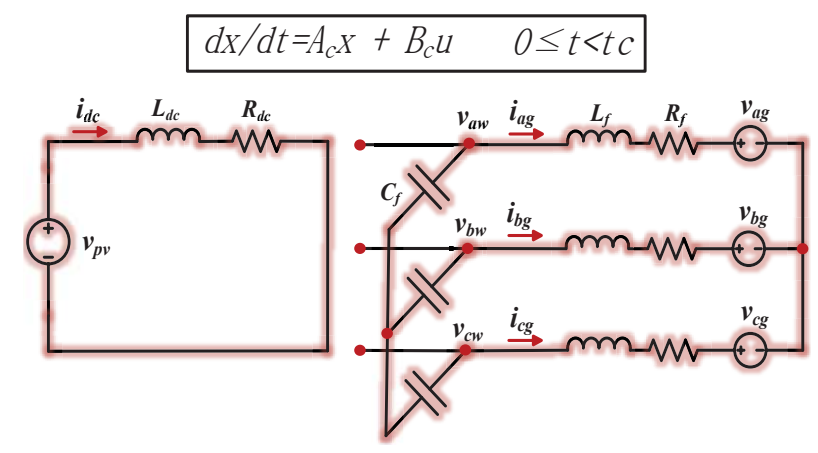

(a)

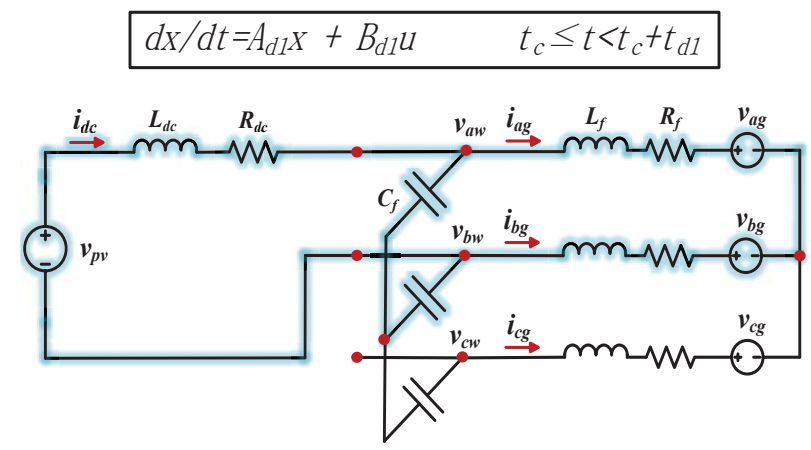

(b)

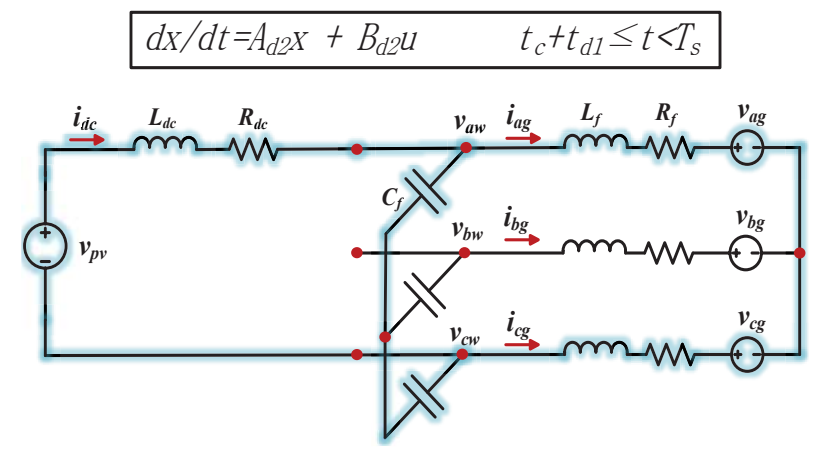

(c)

Figure 5.7: Circuit diagrams and state equation of the three-phase grid-tied CSI-based MIC for Sector I. (a) Charging state, $S_{1}$ and $S_{4}$ on. (b) Discharging state, $S_{1}$ and $S_{6}$ on. (c) Discharging state, $S_{1}$ and $S_{2}$ on.

for all six sectors. Also, it should be noted that the input matrices are the same at any switching state: $B_{c}=B_{d 1}=B_{d 2}=B$. The input matrix, as well as the system matrices in charging and discharging states, can be derived based on the circuit diagrams provided in Figure 5.7, as given by 


$$
\begin{gathered}
B=\left[\begin{array}{ccc}
\frac{1}{L_{f}} & 0 & 0 \\
0 & \frac{-1}{2 L_{f}} & \frac{-\sqrt{3}}{6 L_{f}} \\
0 & \frac{\sqrt{3}}{6 L_{f}} & \frac{-1}{2 L_{f}} \\
0 & 0 & 0 \\
0 & 0 & 0
\end{array}\right], A_{c}=\left[\begin{array}{ccccc}
\frac{-R_{d c}}{L_{d c}} & 0 & 0 & 0 & 0 \\
0 & \frac{2}{3 L_{f}} & \frac{1}{3 L_{f}} & \frac{-R_{f}}{L_{f}} & 0 \\
0 & \frac{-1}{3 L_{f}} & \frac{1}{3 L_{f}} & 0 & \frac{-R_{f}}{L_{f}} \\
0 & 0 & 0 & \frac{-1}{C_{f}} & \frac{1}{C_{f}} \\
0 & 0 & 0 & \frac{-1}{C_{f}} & \frac{-2}{C_{f}}
\end{array}\right] \\
A_{d_{1}}=\left[\begin{array}{ccccc}
\frac{-R_{d c}}{L_{d c}} & \frac{-1}{L_{d c}} & 0 & 0 & 0 \\
0 & \frac{2}{3 L_{f}} & \frac{1}{3 L_{f}} & \frac{-R_{f}}{L_{f}} & 0 \\
0 & \frac{-1}{3 L_{f}} & \frac{1}{3 L_{f}} & 0 & \frac{-R_{f}}{L_{f}} \\
\frac{2}{C_{f}} & 0 & 0 & \frac{-1}{C_{f}} & \frac{1}{C_{f}} \\
\frac{-1}{C_{f}} & 0 & 0 & \frac{-1}{C_{f}} & \frac{-2}{C_{f}}
\end{array}\right], A_{d_{2}}=\left[\begin{array}{ccccc}
\frac{-R_{d c}}{L_{d c}} & \frac{-1}{L_{d c}} & \frac{-1}{L_{d c}} & 0 & 0 \\
0 & \frac{2}{3 L_{f}} & \frac{1}{3 L_{f}} & \frac{-R_{f}}{L_{f}} & 0 \\
0 & \frac{-1}{3 L_{f}} & \frac{1}{3 L_{f}} & 0 & \frac{-R_{f}}{L_{f}} \\
\frac{1}{C_{f}} & 0 & 0 & \frac{-1}{C_{f}} & \frac{1}{C_{f}} \\
\frac{1}{C_{f}} & 0 & 0 & \frac{-1}{C_{f}} & \frac{-2}{C_{f}}
\end{array}\right]
\end{gathered}
$$

The average state matrix of Sector I can be obtained from $A_{I}=\left[d_{c} A_{c}+d_{1} A_{d 1}+\right.$ $\left.d_{2} A_{d 2}\right]$, where $d_{c}=t_{c} / T_{s}, d_{1}=t_{d 1} / T_{s}$, and $d_{2}=t_{d 2} / T_{s}$ are the corresponding duty ratios [172]. Similarly, the averaged system matrices for other sectors, $A_{I I}, A_{I I I}, A_{I V}$, $A_{V}$, and $A_{V I}$ can be obtained from their six sectors of PPWM and corresponding circuit diagrams, respectively.

The averaged system matrix $A_{I}$ varies with time due to the fact that it is a function of charging and discharging intervals (see Equation (5.4)). However, transferring Equation (5.8) to the synchronously rotating $d q$ reference frame results in a time invariant statespace averaging model as follows

$$
d x_{d q} / d t=A_{d q I} x_{d q}+B u_{d q}
$$

where $x_{d q}=\left[\begin{array}{lllll}i_{d c} & i_{d g} & i_{q g} & v_{d w} & v_{q w}\end{array}\right]^{T}$ and $u_{d q}=\left[\begin{array}{lll}v_{p v} & v_{d g} & v_{q g}\end{array}\right]^{T}$ are the approximate state vector and input vector in the rotating $d q$-frame of reference.

It can be proved that the averaged matrices obtained for the six sectors in the $d q$ reference frame are the same, i.e., $A_{d q I}=A_{d q I I}=\cdots=A_{d q V I}=A_{d q}$, in which the $A_{d q}$ 
represents the dynamic matrix of the large-signal model [172]. Therefore, the state-space average representation of the three-phase grid-tied CSI-based MIC system in the rotating $d q$-frame of reference can be written as

$$
\begin{aligned}
& \frac{d}{d t}\left[\begin{array}{c}
i_{d c} \\
i_{d g} \\
i_{q g} \\
v_{d w} \\
v_{q w}
\end{array}\right]=\underbrace{\left[\begin{array}{ccccc}
\frac{-R_{d c}}{L_{d c}} & 0 & 0 & \frac{-\sqrt{3} m_{i} \sin \left(\alpha_{0}\right)}{2 L_{d c}} & \frac{-\sqrt{3} m_{i} \cos \left(\alpha_{0}\right)}{2 L_{d c}} \\
0 & \frac{-R_{f}}{L_{f}} & \omega & \frac{1}{2 L_{f}} & \frac{\sqrt{3}}{6 L_{f}} \\
0 & -\omega & \frac{-R_{f}}{L_{f}} & \frac{-\sqrt{3}}{6 L_{f}} & \frac{1}{2 L_{f}} \\
\frac{\sqrt{3} m_{i} \sin \left(\alpha_{0}\right)}{C_{f}} & \frac{-3}{2 C_{f}} & \frac{\sqrt{3}}{2 C_{f}} & 0 & \omega \\
\frac{\sqrt{3} m_{i} \cos \left(\alpha_{0}\right)}{C_{f}} & \frac{-\sqrt{3}}{2 C_{f}} & \frac{-3}{2 C_{f}} & -\omega & 0
\end{array}\right]}_{A_{d q}}\left[\begin{array}{c}
i_{d c} \\
i_{d g} \\
i_{q g} \\
v_{d w} \\
v_{q w}
\end{array}\right]+ \\
& \underbrace{\left[\begin{array}{ccc}
\frac{1}{L_{d c}} & 0 & 0 \\
0 & \frac{-1}{2 L_{f}} & \frac{-\sqrt{3}}{6 L_{f}} \\
0 & \frac{\sqrt{3}}{6 L_{f}} & \frac{-1}{2 L_{f}} \\
0 & 0 & 0 \\
0 & 0 & 0
\end{array}\right]}_{B}\left[\begin{array}{l}
v_{p v} \\
v_{d g} \\
v_{q g}
\end{array}\right]
\end{aligned}
$$

The 5th order state-space model in (5.12) can be divided into $q$-axis and $d$-axis equations on the ac side and dc side. The CSI's dynamics of the dc-side input can be written as

$$
v_{p v}=L_{d c} \frac{d i_{d c}}{d t}+R_{d c} i_{d c}+\frac{\sqrt{3}}{2} m_{i} \sin \left(\alpha_{0}\right) v_{d w}+\frac{\sqrt{3}}{2} m_{i} \cos \left(\alpha_{0}\right) v_{q w}
$$

The dc voltage equation in (5.13) contains both $d$-axis and $q$-axis components, which couple the dc input to the ac output. The $d$-axis dynamics at ac side can be expressed as

$$
\begin{gathered}
i_{d g}=\frac{2 \sqrt{3}}{3} m_{i} \sin \left(\alpha_{0}\right) i_{d c}+\frac{\sqrt{3}}{3} i_{q g}+\frac{2}{3} C_{f} \omega v_{q w}-\frac{2}{3} C_{f} \frac{d v_{d w}}{d t} \\
v_{d w}=2 L_{f} \frac{d i_{d g}}{d t}+2 R_{f} i_{d g}-2 L_{f} \omega i_{q g}-\frac{\sqrt{3}}{3} v_{q w}+v_{d g}+\frac{\sqrt{3}}{3} v_{q g}
\end{gathered}
$$


Both of the above equations contain the $q$-axis components. These terms can be modeled as dependent current and voltage sources. Similarly, at the ac-side, the $q$-axis dynamics including dependent sources can be obtained as

$$
\begin{gathered}
i_{q g}=\frac{2 \sqrt{3}}{3} m_{i} \cos \left(\alpha_{0}\right) i_{d c}-\frac{\sqrt{3}}{3} i_{d g}-\frac{2}{3} C_{f} \omega v_{d w}-\frac{2}{3} C_{f} \frac{d v_{q w}}{d t} \\
v_{q w}=2 L_{f} \frac{d i_{q g}}{d t}+2 R_{f} i_{q g}+2 L_{f} \omega i_{d g}+\frac{\sqrt{3}}{3} v_{d w}+v_{q g}+\frac{\sqrt{3}}{3} v_{d g}
\end{gathered}
$$

The average equivalent circuit model of the single-stage three-phase CSI in the stationary $d q$ frame is shown in Figure 5.8. The equivalent circuits at dc-side, $d$-axis, and $q$-axis are modeled by passive elements, as well as dependent voltage and current sources. The stability and sensitivity of the three-phase grid-tied CSI-based MIC system can be studied using these equivalent models.

\subsubsection{Small-Signal Model}

As expressed in (5.12), $u=\left[\begin{array}{lll}v_{p v} & v_{d g} & v_{q g}\end{array}\right]^{T}$ represents the system input for constant values of $m_{i}$ and $\alpha_{0}$. While for the practical implementation of the proposed system, $m_{i}$ and $\alpha_{0}$ can be used to regulate the injected active and reactive powers to the grid, in grid-tied conditions. Thus, (5.12) should be linearized around a steady-state operating point by defining small perturbations for the system variables as

$$
x_{d q}=X_{d q}+\delta x_{d q}, \quad v_{p v}=V_{p v}+\delta v_{p v}, \quad m_{i}=M_{i}+\delta m_{i}, \quad \alpha_{0}=A_{0}+\delta \alpha_{0}
$$

where the symbol $\delta$ denotes small-signal perturbation, and the capital letters indicate their steady-state components. The linearized (small-signal) model can be written as (5.19). The stability studies of the small signal model in (5.19) can be performed by using several different techniques. However, the eigenvalue analysis is one of the common techniques to study stability of the system as explained in the following subsection. 


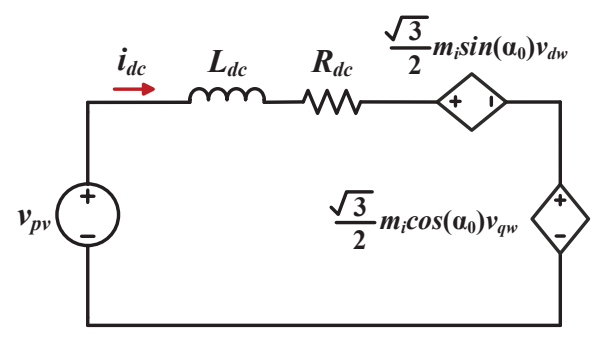

(a)

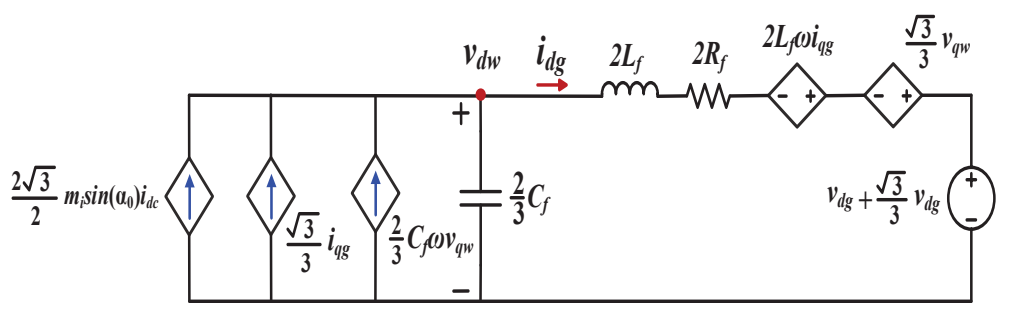

(b)

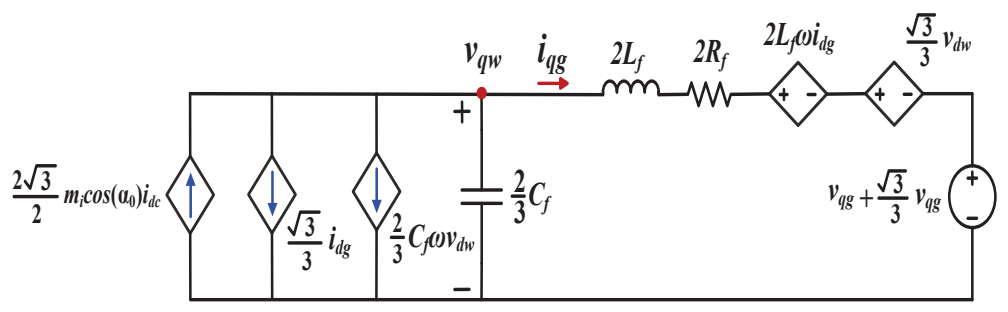

(c)

Figure 5.8: CSI-based MIC equivalent circuits. (a) dc-side equivalent circuit. (b) $d$-axis equivalent circuit. (c) $q$-axis equivalent circuit.

$$
\begin{aligned}
& \frac{d}{d t}\left[\begin{array}{l}
\delta i_{d c} \\
\delta i_{d g} \\
\delta i_{q g} \\
\delta v_{d w} \\
\delta v_{q w}
\end{array}\right]=\left[\begin{array}{ccccc}
\frac{-R_{d c}}{L_{d c}} & 0 & 0 & \frac{-\sqrt{3} M_{i} \sin \left(A_{0}\right)}{2 L_{d c}} & \frac{-\sqrt{3} M_{i} \cos \left(A_{0}\right)}{2 L_{d c}} \\
0 & \frac{-R_{f}}{L_{f}} & \omega & \frac{1}{2 L_{f}} & \frac{\sqrt{3}}{6 L_{f}} \\
0 & -\omega & \frac{-R_{f}}{L_{f}} & \frac{-\sqrt{3}}{6 L_{f}} & \frac{1}{2 L_{f}} \\
\frac{\sqrt{3} M_{i} \sin \left(A_{0}\right)}{C_{f}} & \frac{-3}{2 C_{f}} & \frac{\sqrt{3}}{2 C_{f}} & 0 & \omega \\
\frac{\sqrt{3} M_{i} \cos \left(A_{0}\right)}{C_{f}} & \frac{-\sqrt{3}}{2 C_{f}} & \frac{-3}{2 C_{f}} & -\omega & 0
\end{array}\right]\left[\begin{array}{c}
\delta i_{d c} \\
\delta i_{d g} \\
\delta i_{q g} \\
\delta v_{d w} \\
\delta v_{q w}
\end{array}\right]+ \\
& {\left[\begin{array}{ccccc}
\frac{1}{L_{d c}} & 0 & 0 & \frac{\sqrt{3}}{2 L_{d c}}\left[\sin \left(A_{0}\right) V_{d w}-\cos \left(A_{0}\right) V_{q w}\right] & \frac{\sqrt{3} M_{i}}{2 L_{d c} T_{s}}\left[\cos \left(A_{0}\right) V_{d w}+\sin \left(A_{0}\right) V_{q w}\right] \\
0 & \frac{-1}{2 L_{f}} & \frac{-\sqrt{3}}{6 L_{f}} & 0 & 0 \\
0 & \frac{\sqrt{3}}{6 L_{f}} & \frac{-1}{2 L_{f}} & 0 & 0 \\
0 & 0 & 0 & \frac{-\sqrt{3} \sin \left(A_{0}\right) I_{d c}}{C_{f}} & \frac{-\sqrt{3} M_{i} \cos \left(A_{0}\right) I_{d c}}{C_{f}} \\
0 & 0 & 0 & \frac{\sqrt{3} \cos \left(A_{0}\right) I_{d c}}{C_{f}} & \frac{-\sqrt{3} M_{i} \sin \left(A_{0}\right) I_{d c}}{C_{f}}
\end{array}\right]\left[\begin{array}{l}
\delta v_{p v} \\
\delta v_{d g} \\
\delta v_{q g} \\
\delta m_{i} \\
\delta \alpha_{0}
\end{array}\right]}
\end{aligned}
$$


Table 5.1: System Parameters

\begin{tabular}{llll}
\hline \hline Parameters & Value & Parameters & Value \\
\hline \hline Rated power & $300 \mathrm{VA}$ & dc-link resistance & $R_{d c}=0.2 \Omega$ \\
Rated phase voltage & $120 \mathrm{~V}$ & dc-link inductance & $L_{d c}=6 \mathrm{mH}$ \\
Rated frequency & $60 \mathrm{~Hz}$ & Filter capacitance & $C_{f}=15 \mu \mathrm{F}$ \\
Switching frequency & $3 \mathrm{kHz}$ & Filter inductance & $L_{f}=2 \mathrm{mH}$ \\
dc-link voltage & $50 \mathrm{~V}$ & Filter resistance & $R_{f}=2 \Omega$ \\
\hline \hline
\end{tabular}

\subsubsection{Open-Loop Stability Analysis}

Herein, the stability of the proposed system, with respect to the variation in system matrix parameters of the equation (5.19), is studied. This study is based on a 300-VA laboratory prototype, in which parameters are summarized in Table 5.1. In addition, the grid voltage frequency is assumed constant at $\omega=120 \pi$ and modulations are initially set at $m_{i}=0.2$ and $\alpha_{0}=-\pi / 6$. Substituting these values into (5.19) results in the state vector at equilibrium point as $X_{d q}=\left[\begin{array}{lllll}61.46 & 0.48 & 12.16 & 34.82 & 148.57\end{array}\right]^{T}$, which represents the steady-state values of the state vector $x_{d q}$ at a system operating point of about $300 \mathrm{~W}$ and 0 Var (rms grid current of $0.86 \mathrm{~A}$ ). The eigenvalues of the system at this operating point are $\lambda_{1}=-101.6, \lambda_{2,3}=-867.1 \pm j 6065.9$, and $\lambda_{4,5}=-123.7 \pm j 4718.3$. The real parts of all five eigenvalues are negative, which signifies the linearized system's stability. However, the stability of the overall system cannot be guaranteed by system matrix parameters. Thus, for stability analysis, the eigenvalues of the system are plotted versus the variation of one parameter at a time, while the other nominal parameters remain constant. It is worth mentioning that the system matrix in (5.19) is independent of $v_{p v}$ and therefore, the system eigenvalues remain constant with the variations of the dc-link voltage. In fact, the variation of the dc-link voltage does not affect the stability of the proposed system. 


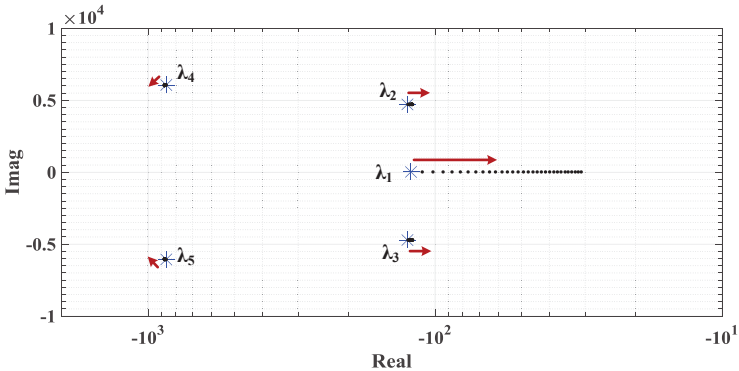

(a)

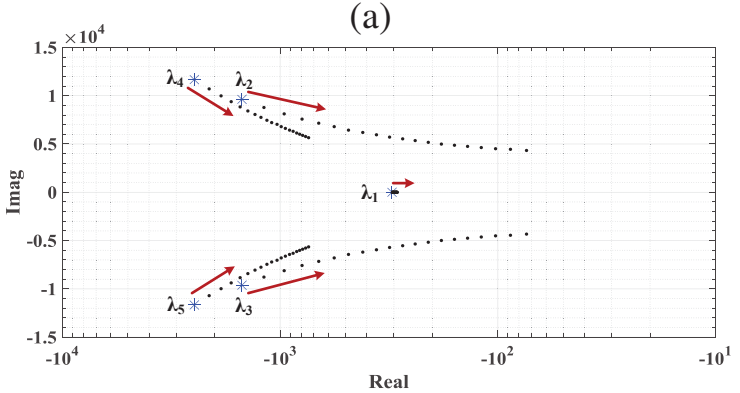

(c)

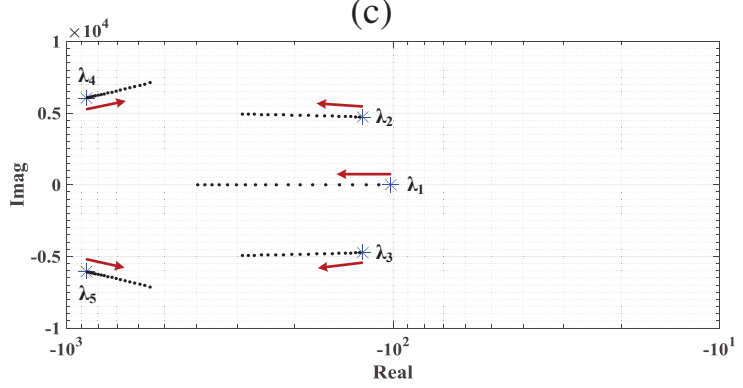

(e)

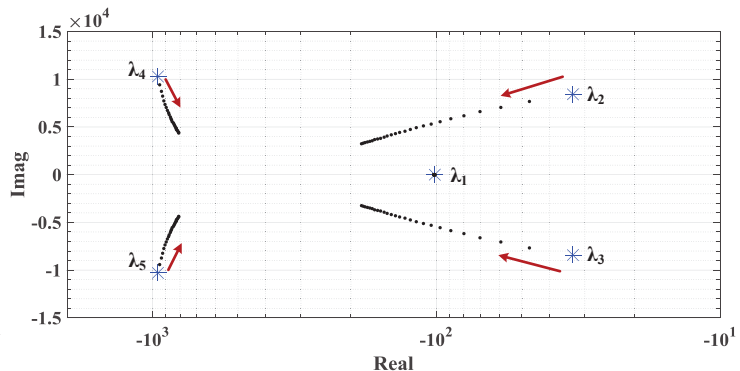

(b)

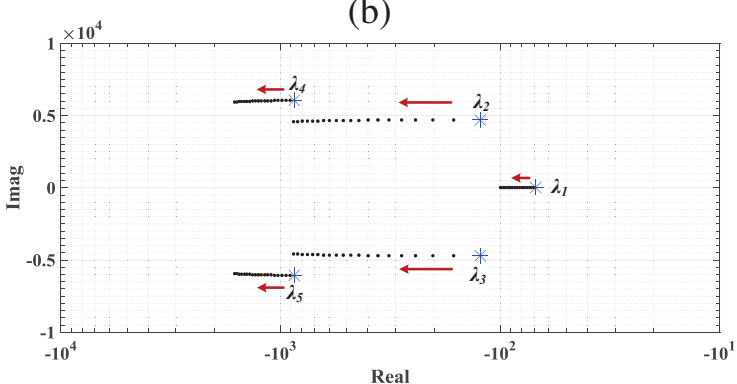

(d)

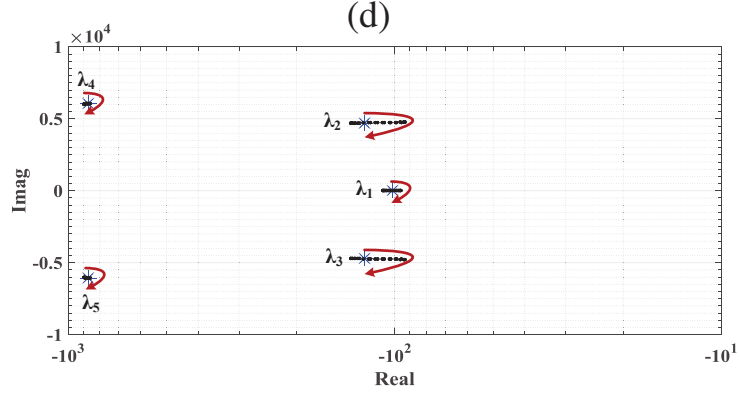

(f)

Figure 5.9: Location of eigenvalues of small-signal model. (a) $L_{d c}$ varies from $5 \mathrm{mH}$ to $20 \mathrm{mH}$. (b) $C_{f}$ varies from $5 \mu \mathrm{F}$ to $30 \mu \mathrm{F}$. (c) $L_{f}$ varies from $0.5 \mathrm{mH}$ to $2.5 \mathrm{mH}$. (d) $R_{f}$ varies from $2 \Omega$ to $5 \Omega$. (e) $m_{i}$ varies from 0.2 to 1 . (e) $\alpha_{0}$ varies from $-\pi / 6$ to $11 \pi / 6$.

\section{1) Eigenvalues Movement for Variations in $L_{d c}$}

The impacts of $L_{d c}$ on the eigenvalues of the system are shown in Figure 5.9(a), when $L_{d c}$ varies from $5 \mathrm{mH}$ to $20 \mathrm{mH}$. It can be observed that the eigenvalues of $\lambda_{2}, \lambda_{3}, \lambda_{4}$, and $\lambda_{5}$, slightly change, while $\lambda_{1}$ moves toward right, staying in the left-half of the s-plane. Thus, the system is stable with variations in the dc-link inductance. It should be noted that rising the $L_{d c}$, consequently increasing the $R_{d c}$, increases the losses at the dc side, which is usually undesirable for PV MIC systems. 


\section{2) Eigenvalues Movement for Variations in $C_{f}$}

The variation of system eigenvalues are shown in Figure 5.9(b), in which the filter capacitor value varies from $5 \mu \mathrm{F}$ to $30 \mu \mathrm{F}$, with other nominal parameter values provided in Table I. It is obvious that the complex eigenvalues $\lambda_{2}, \lambda_{3}, \lambda_{4}$, and $\lambda_{5}$ move toward the real axis, whereas the impact of the $C_{f}$ on the eigenvalue $\lambda_{1}$ is almost negligible. This reduces the system's natural frequencies and increases the damping factor of the system. However, the larger value of the filter capacitor might lead to a much lower power factor in the system.

\section{3) Eigenvalues Movement for Variations in $\mathbf{L}_{f}$ and $\mathbf{R}_{f}$}

The impact of the filter inductance and resistance on the location of the system eigenvalues are demonstrated in Figure 5.9(c) and 5.9(d), respectively, as $L_{f}$ varies from $0.5 \mathrm{mH}$ to $2.5 \mathrm{mH}$, and $R_{f}$ changes from $2 \Omega$ to $5 \Omega$. It can be observed from Figure 5.9 (c) that the increase of the filter inductance displaces all complex eigenvalues toward the vertical axis of the s-plan. It is expected that the overall system response becomes longer and less oscillatory as $L_{f}$ increases. However, since the filter inductance reduces the injected current harmonics to the grid, it may not be a good idea to decrease its value to improve the system's stability, without first considering the quality of the current waveform.

On the contrary, as can be seen in Figure 5.9(d), increasing the resistance of the filter moves all eigenvalues of the system toward the left, resulting in a faster response to any step changes in the control signals. However, increase of the resistance increases the power losses of the system, which is usually undesirable.

\section{4) Eigenvalues Movement for Variations in $\mathbf{m}_{\mathbf{i}}$}

Figure 5.9(e) illustrates the small signal poles (eigenvalues) of the CSI-based MIC system as modulation index $m_{i}$ varies from 0.2 to 1 . This range of $m_{i}$ corresponds to the apparent power variation from 0 to $300 \mathrm{VA}$. As can be seen, the imaginary part of $\lambda_{4}$ and $\lambda_{5}$ 
increase, while the real parts decrease as $m_{i}$ increases. Unlike $\lambda_{4}$ and $\lambda_{5}$, the values of the $\lambda_{1}, \lambda_{2}$, and $\lambda_{3}$ move away from the real axis as $m_{i}$ increases. Since the eigenvalues $\lambda_{1}, \lambda_{2}$, and $\lambda_{3}$ are dominant than $\lambda_{4}$ and $\lambda_{5}$, it can be expected that the overall system response becomes faster with less oscillatory. Nevertheless, it is obviously demonstrated that all eigenvalues stay on the left half of the s-plane for the whole operating range, which presents the stability of the CSI-based MIC system over the range.

\section{5) Eigenvalues Movement for Variations in $\alpha_{0}$}

The system eigenvalues, as the modulation angle $\alpha_{0}$ varies during one grid frequency cycle (from $-\pi / 6$ to $11 \pi / 6$ ), are shown in Figure 5.9(f). Although the system matrix in (5.19) is a function of $\alpha_{0}$, it is surprising that the eigenvalues of the system are only able to rotationally displace as the modulation angle varies. Thus, it will never affect the stability of the proposed system.

\subsection{Power Control System for Three-Phase CSI-Based MIC}

Several maximum power point tracking (MPPT) algorithms have been reported for the PV inverter studies [173]. The outcome of MPPT provides the active power reference for the CSI. This section mainly focuses on the converter control; hence, the MPPT control method is not discussed. A multivariable PI-based $d q$ current controllers with feedback decoupling capability are proposed in which the step changes in one axis negligibly affect the other. A PPWM switching pattern for the CSI-based MIC system is employed to produce charging and discharging intervals to generate gating signals. The reactive and active powers of the PV MIC system can be independently regulated by controlling the modulation index $m_{i}$ and the modulation angle $\alpha_{0}$. Compared with other modulation schemes, PPWM can provide more control flexibility and better dynamic performance [169]. 


\subsubsection{Comparative Study of Conventional and Multivariable PI-Based}

\section{$d q$ Current Control}

The conventional $d q$ current control strategy is well known and has been widely studied in the literature [65-67]. In order to achieve the decoupled control of $i_{d g}$ and $i_{q g}$, the output voltages of the converter should be controlled by adopting (5.15) and (5.17) in Section 5.3 , as follows

$$
\left\{\begin{array}{l}
v_{d w}=u_{c d}-2 L_{f} \omega i_{q g}+v_{e} \\
v_{q w}=u_{c q}+2 L_{f} \omega i_{d g}+v_{f}
\end{array}\right.
$$

where $u_{c d}$ and $u_{c q}$ are control signals in the $d$ and $q$ axes in the rotating reference frame,

respectively, and the voltages $v_{e}=-\frac{\sqrt{3}}{3} v_{q w}+v_{d g}+\frac{\sqrt{3}}{3} v_{q g}$ and $v_{f}=\frac{\sqrt{3}}{3} v_{d w}+v_{q g}+\frac{\sqrt{3}}{3} v_{d g}$ are voltage feedforwards. According to (5.20), the structural diagram of the current regulator based on conventional PI controllers is depicted in Figure 5.10(a), in which the voltage feedforwards and the coupling terms are shown. A conventional PI controller can be adopted in form of the Laplace transformation $P(s)$, given by

$$
P(s)=\frac{k_{p} s+k_{I}}{s}
$$

where, $k_{p}$ and $k_{I}$ are the proportional gain and integral gain of the controller, respectively.

It should be noted that a fully decoupled system can be theoretically produced by adopting the feedforward signals. If the feedforward signals can accurately neutralize the effect of the coupling terms (i.e., $\omega L_{f} i_{d g}$ and $\omega L_{f} i_{q g}$ ), the complete decoupling can be accomplished. However, due to measurement errors in a real system, it is not practically possible to precisely determine the value of $i_{d g}, i_{q g}$, and $L_{f}$. Furthermore, the sampling process might produce a delay in the system, resulting in an imperfect cancellation. Thus, implementing the conventional PI control strategy cannot fully decouple the axis in a practical case. Compared with the conventional PI-based control method, which relies 


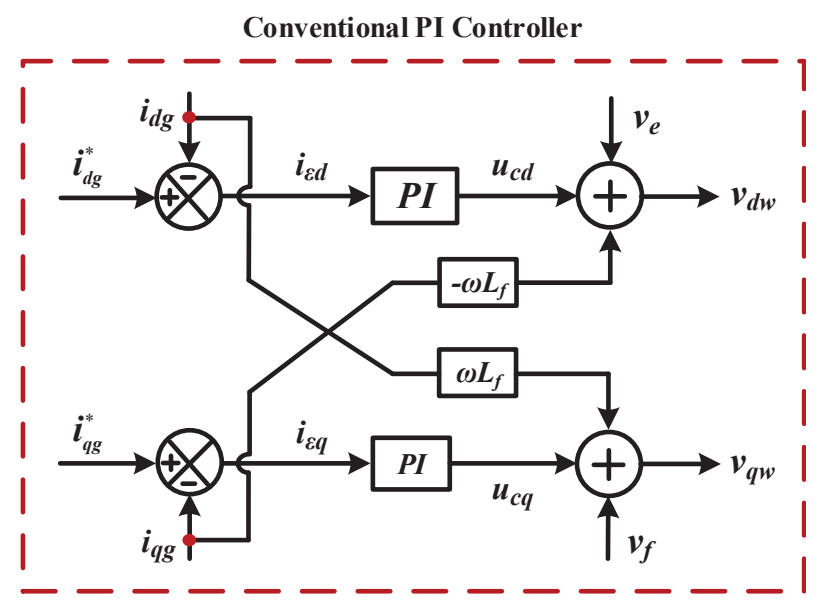

(a)

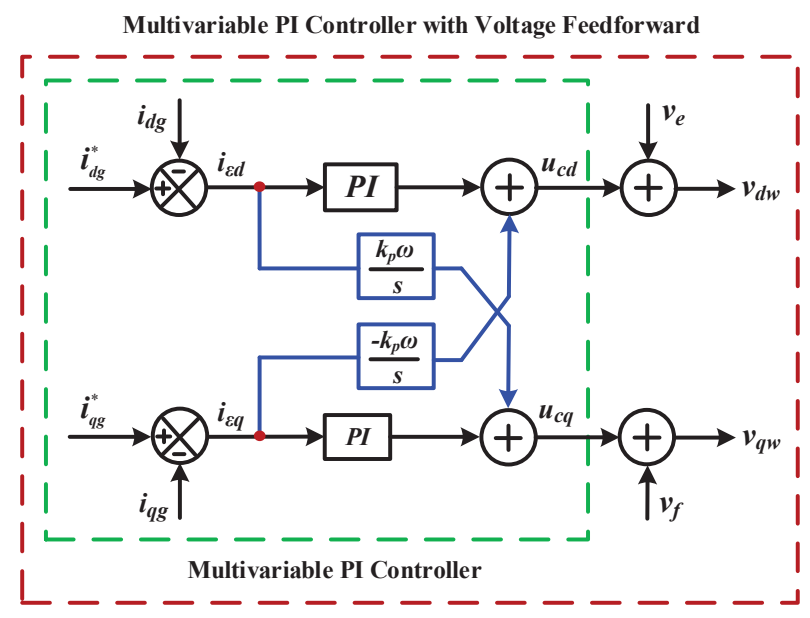

(b)

Figure 5.10: Structural diagram of $d q$ current control. (a) Conventional PI-based control. (b) Multivariable PI-based control with voltage feedforward.

on the feedforward signals to exclude the coupling effects, the proposed multivariable PIbased technique employs the plant inversion approach to design a fully decoupled control system. The multivariable PI controller can be defined as [68]

$$
P(s)=\frac{\left[k_{p}(s+j \omega)+k_{I}\right]}{s}
$$

where it has a complex transfer function. When separating the real and imaginary parts, the controller can be rewritten as 


$$
u_{c d}+j u_{c q}=\left(\frac{k_{p} s+k_{I}}{s}+j \frac{k_{p} \omega}{s}\right)\left(i_{\varepsilon d}+j i_{\varepsilon q}\right)
$$

Therefore

$$
\begin{aligned}
& u_{c d}=\frac{k_{p} s+k_{I}}{s} i_{\varepsilon d}-\frac{k_{p} \omega}{s} i_{\varepsilon q} \\
& u_{c q}=\frac{k_{p} s+k_{I}}{s} i_{\varepsilon q}+\frac{k_{p} \omega}{s} i_{\varepsilon d}
\end{aligned}
$$

where, $i_{\varepsilon d}$ and $i_{\varepsilon q}$ are the error signals of the $d$ and $q$ current axes in the rotating reference frame, respectively. The control signals in both the $d$ and $q$ axes are described in (5.24) and (5.25), including cross-coupling terms. However, dissimilar to the conventional PI

controller, the coupling terms (i.e., $\frac{k_{p} \omega}{s} i_{\varepsilon q}$ and $\frac{k_{p} \omega}{s} i_{\varepsilon d}$ ) include integrators, which can considerably decrease the coupling effect of the axis on each other. Figure 5.10(b) shows a structural diagram of the proposed multivariable PI controller resulting in a practically decoupled axis.

\subsubsection{Active and Reactive Power Control Implementation}

Details of the proposed power control scheme for the three-phase grid-tied CSI-based MIC system are illustrated in Figure 5.11. The major control objectives include the following: 1) tracking of the reference dc-link current; 2) providing a certain amount of active and reactive power; and 3) significantly decoupling the active and reactive powers from each other, upon the step changes in command signals.

The controller is combined with two independent control loops for active and reactive powers, respectively. In order to achieve independent control, the three-phase grid currents $i_{a b c}^{g}$ are transformed into the $d q$ components $i_{d g}$ and $i_{q g}$ in the grid-voltage reference frame. The grid-voltage phase-locked loop (PLL) is employed to obtain a synchronous angle $\theta_{g}=\omega t$. With the $d q$ synchronous frame, the grid voltages $v_{a b c}^{g}$ only have the $d$-axis component $v_{d g}$ while the $q$-axis component $v_{q g}$ equals zero. Thus, the active and reactive 

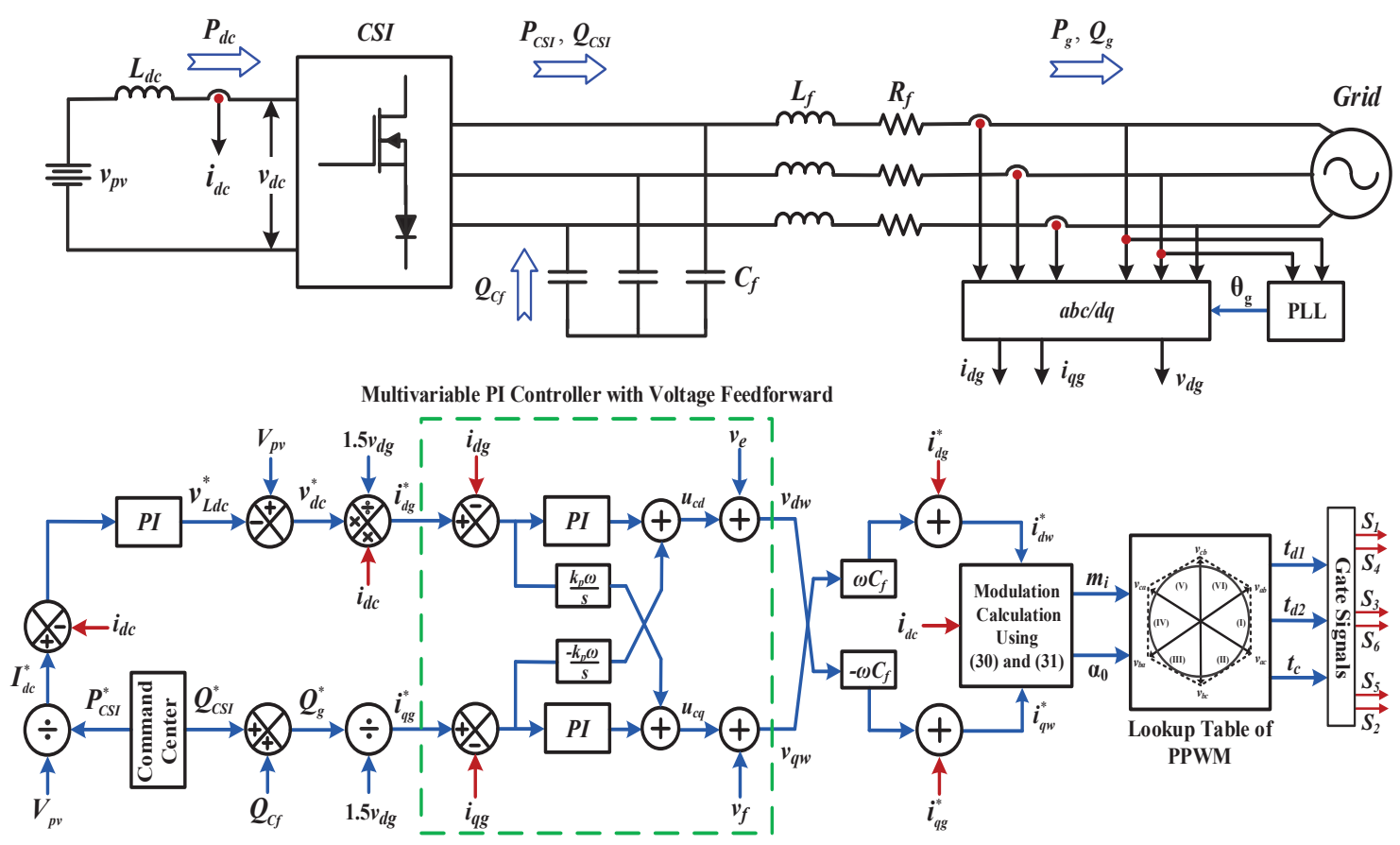

Figure 5.11: Overall control block diagram of the three-phase grid-tied CSI-based MIC system.

power exchanged from the PV MIC system to the grid can be independently controlled by regulating the grid currents $i_{d g}$ and $i_{q g}$, respectively, as

$$
\left\{\begin{array}{l}
P_{g}=1.5 v_{d g} i_{d g} \\
Q_{g}=1.5 v_{d g} i_{q g} .
\end{array}\right.
$$

By neglecting the loss in the system, the active power on the grid side is equal to the active power of the dc-link in a steady-state operation, which is given by

$$
P_{g}=P_{C S I}=P_{d c}=V_{p v} I_{d c}
$$

where $V_{p v}$ and $I_{d c}$ are the dc-link voltage and current magnitude, respectively. The dc-link current is controlled so that the CSI can supply the required active power to the grid. Thus, the PI controller with a control speed of $T_{s} / L_{d c}$ is used to generate the $d$-axis reference of the grid current [174]. The magnitude of the dc-link current reference $I_{d c}^{*}$, which can be 
determined from (5.27), is compared with the measured dc-link current $i_{d c}$, and an error signal is input to the PI regulator to generate a control signal of $v_{L d c}^{*}$. The dc voltages of the PV side and converter side, along with the voltage across the dc-link inductance, satisfy the equation $v_{d c}^{*}=V_{p v}-v_{L d c}^{*}$. By neglecting the loss in the system, the grid reference current $i_{d g}^{*}$ is derived based on the power-balance assumption, i.e.,

$$
i_{d g}^{*}=\frac{P_{g}^{*}}{1.5 v_{d g}}=\frac{v_{d c}^{*} i_{d c}}{1.5 v_{d g}} .
$$

The grid reactive power can be adjusted through proper control of the $q$-component current. The filter capacitor can be selected to inject a fixed amount of the reactive power $Q_{C_{f}}$, which is about 0.25 p.u. of the rated power (in this study). The $q$-axis grid reference current $i_{q g}^{*}$ can simply be obtained by using (5.26), where $Q_{C_{f}}$ is added into the CSI reactive power command $Q_{C S I}^{*}$ in order to find the total reactive power reference $Q_{g}^{*}$ injected into the grid.

The measured signals of the grid's active and reactive currents, $i_{d g}$ and $i_{q g}$, are compared with their reference values, and then error signals are passed through the proposed multivariable PI controller with voltage feedforward, shown in Figure 5.10(b), to derive the converter output voltages.

The steady-state $d q$ components of the capacitor currents, derived by using converter output voltages, are $i_{d c_{f}}=-\omega C_{f} v_{q w}$ and $i_{q c_{f}}=\omega C_{f} v_{d w}$. Thus, the output reference current provided by the converter is the subtraction of the grid reference current from the capacitor current, which is written in the $d q$ axes as

$$
\left\{\begin{array}{l}
i_{d w}^{*}=i_{d g}^{*}-i_{d c_{f}}=i_{d g}^{*}+\omega C_{f} v_{q w} \\
i_{q w}^{*}=i_{q g}^{*}-i_{q c_{f}}=i_{q g}^{*}-\omega C_{f} v_{d w}
\end{array}\right.
$$

Finally, the currents of $i_{d w}^{*}$ and $i_{q w}^{*}$ are applied to (5.30) and (5.31) to produce the value 


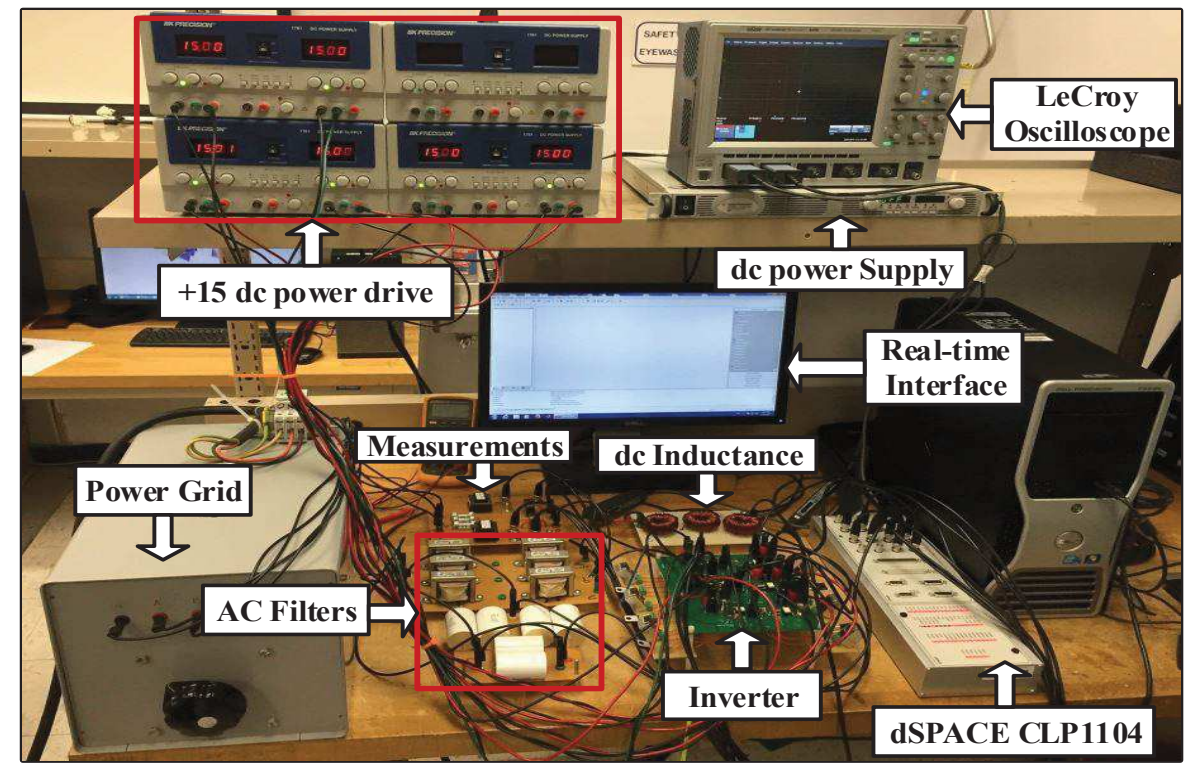

Figure 5.12: Layout of experimental setup.

of the modulation index $m_{i}$ and the modulation angle $\alpha_{0}$, namely control parameters as

$$
\begin{gathered}
m_{i}=\frac{\sqrt{i_{d w}^{*}{ }^{2}+i_{q w}^{*}{ }^{2}}}{i_{d c}} \\
\alpha_{0}=\arccos \left(\frac{2}{3 m_{i} \beta}\right)-\frac{\pi}{6} .
\end{gathered}
$$

To achieved all pre-defined control objectives, the three-phase grid-tied CSI-based MIC uses the PPWM lookup table (discussed in Section II) to generate time intervals of charging and discharging for each sector, i.e., $t_{c}, t_{d 1}, t_{d 2}$ using (5.4), while these time intervals must follow the angle $\theta_{g}=\omega t$.

\subsection{Simulation and Experimental Verification}

The proposed CSI-based MIC system with the multivariable-PI-based power control strategy were first simulated in Matlab/Simulink. Then, to evaluate the performance of the proposed control system, a 300-VA grid-tied prototype of the three-phase, single-stage CSI was built, as shown in Figure 5.12. The main system parameters used in experimental 
work have been listed in Table 5.1. A full-bridge inverter was constructed by Fairchild MOSFETs with low Rds(on) and DSEP 30 diodes, including the snubber protection circuits. The input voltage of this module is provided by a 50-V power supply, connected to the dc-link inductor with $L_{d c}=6 \mathrm{mH}$ and $R_{d c}=0.2 \Omega$. The inverter is connected to the grid (3ph, $208 \mathrm{~V}$ line to line, $60 \mathrm{~Hz}$ ) through the RLC filter unit. The PPWM strategy was applied with the switching frequency of $3 \mathrm{kHz}$. The switching frequency was selected to meet the total harmonic distortion (THD) criteria based on IEEE STD 5191992. It is worth mentioning that the boost ratio in PPWM strategy was kept at about $3.4\left(\beta=V_{m} / V_{p v}=170 / 50=3.4\right)$. The proposed control strategy was implemented by a dSPACE CLP1104 rapid prototyping board, in which the MATLAB/Simulink and dSPACE Control Desk were used together to apply signal processing algorithms. The PI controller for dc-link current was set at $k_{p}=0.005$ and $k_{I}=0.03$, while $k_{p}$ and $k_{I}$ for PI active and reactive current controllers were set at 0.001 and 0.15 , respectively. The measurements were performed using a LeCroyWaverunner 64XI oscilloscope with a bandwidth of $600 \mathrm{MHz}$ and carried out to demonstrate the performance of the proposed control system.

\subsubsection{Simulation Results}

In order to show the dynamic responses of the proposed control system, two scenarios were considered based on the falls and jumps in the CSI-based MIC active and reactive power commands: 1$)$ a step-down in the active power reference value $\left(P_{C S I}^{*}\right)$, and a stepup in the reactive power command at the same time and 2) a step-up in the active power command, while the reactive power reference value has a step-down at the same time. It should be noted that the desired value of the $P_{C S I}^{*}$ is normally dictated by the MPPT algorithm, while the desired value of the $Q_{C S I}^{*}$ can be chosen according to the power system needs. 


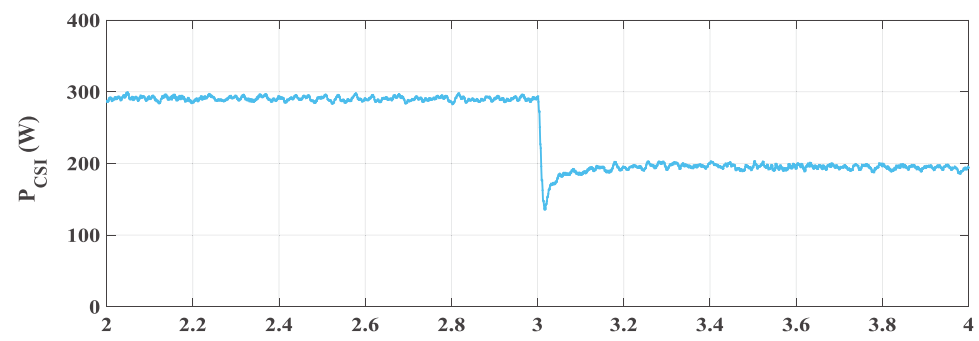

(a)

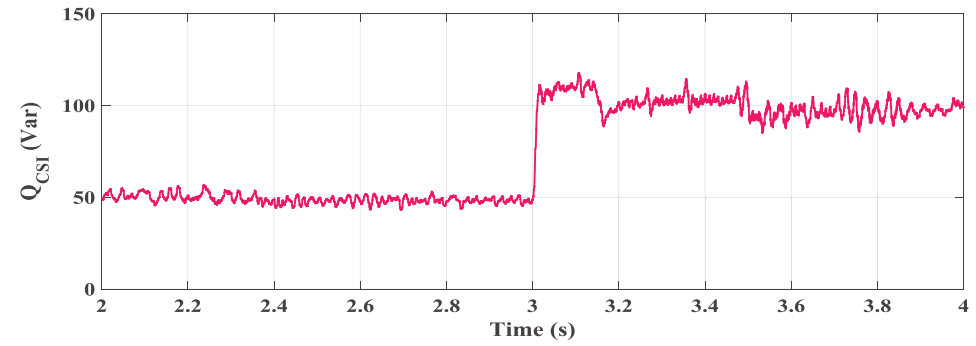

(b)

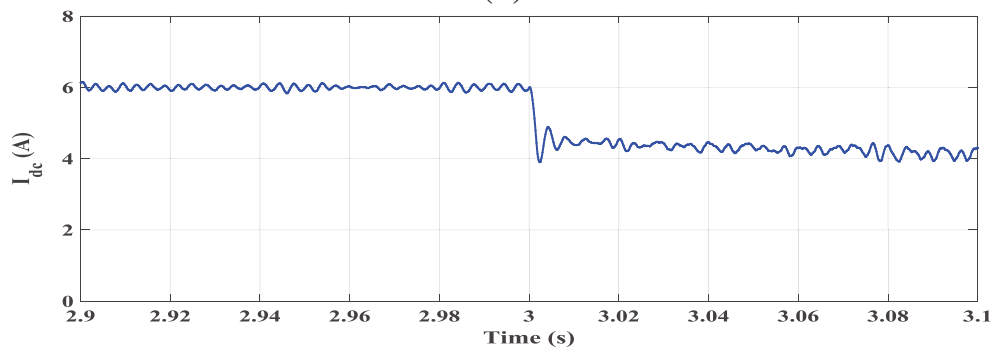

(c)
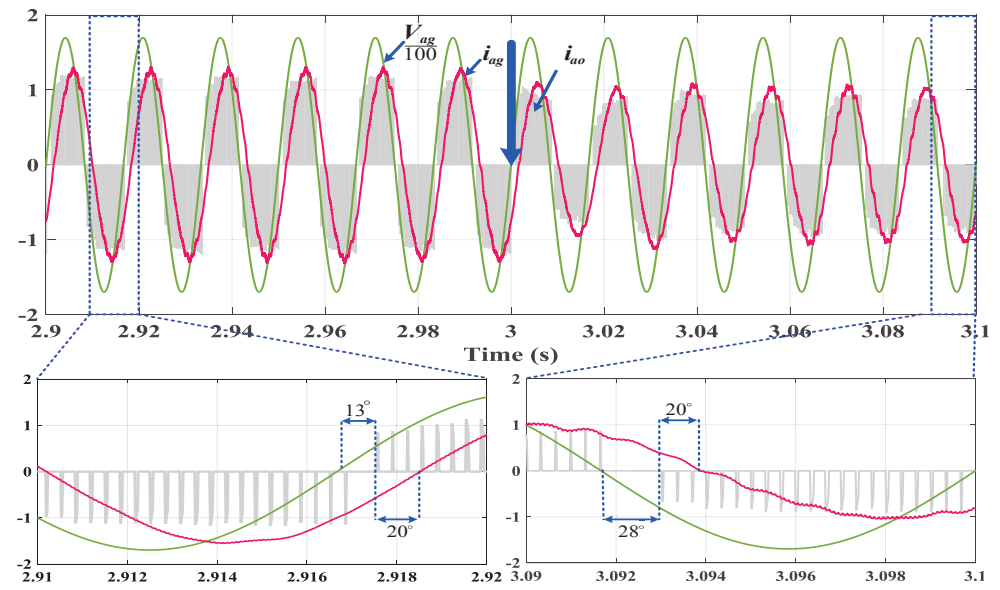

(d)

Figure 5.13: Performance of the proposed method in tracking active and reactive power changes for scenario I. (a) active power step-up. (b) reactive power step-down. (c) dc-link current. (d) voltage and current waveforms. 
Simulation results are shown in Figure 5.13(a)-(d) and Figure 5.14(a)-(d). In the first scenario, the active power was assumed to have a step-down from 300 to $200 \mathrm{~W}$ at $t=3 \mathrm{~s}$, while the reactive power command had a step-up from 50 to 100 Var at the same time of the simulation, indicated in Figure 5.13(a) and 5.13(b). As can be seen, the proposed controller has a promising tracking response to regulate the active and reactive power independently. As displayed in Figure 5.13(c), the dc current is decreased from around $6 \mathrm{~A}$ to $4 \mathrm{~A}$ due to the step change of the CSI active power command. It is observed that the proposed multivariable PI controller has a faster dynamic response and superior axis decoupling capability.

Figure 5.13(d) shows the phase-a waveforms of the grid voltage $v_{a g}$, CSI output current $i_{a o}$, and the grid current $i_{a g}$ for the first scenario. Initially, the CSI current magnitude and its phase angle with respect the $v_{a g}$, which can be obtained from the $\varphi_{\text {inv }}=\arctan \left(Q_{C S I}^{*} / P_{C S I}^{*}\right)$, are $1.2 \mathrm{~A}$ and $13^{0}$, respectively. At $t=3 \mathrm{~s}$ the magnitude of the CSI current steps down to $0.88 \mathrm{~A}$ while phase angle $\varphi_{i n v}$ increases to $28^{\circ}$, as illustrated in Figure 5.13(d).

As demonstrated in overall control system of Figure 5.11, it is expected that for first scenario $m_{i}$ is slightly changed from 0.2 to 0.22 in order to reduce the delivered active power to the grid, while $\alpha_{0}$ is adjusted accordingly to increase reactive power, changing from $-0.297 \mathrm{rad}\left(-17^{0}\right)$ to $-0.035 \mathrm{rad}\left(-2^{0}\right)$. Based on $(5.16)$, it should be note that there is a phase shift $30^{\circ}$ between the $\varphi_{i n v}$ and $\alpha_{0}$, i.e., $\alpha_{0}=\varphi_{i n v}-30^{0}$. As was mentioned in the previous section, the filter capacitor is selected to inject $25 \%$ of rated power (around 80 Var), therefore creating a fixed shift angle of $20^{\circ}$ in the grid current $i_{a g}$. In the second scenario shown in Figure 5.14, the active power command jumps simultaneously from 100 to around $280 \mathrm{~W}$ and reactive power command drops from 200 to 100 VAR at $t=3 \mathrm{~s}$. As shown in Figure 5.14(c), the dc current is increased from 2 A to around 6 A due to the step-up change of the CSI active power command. The proposed controller has shown 


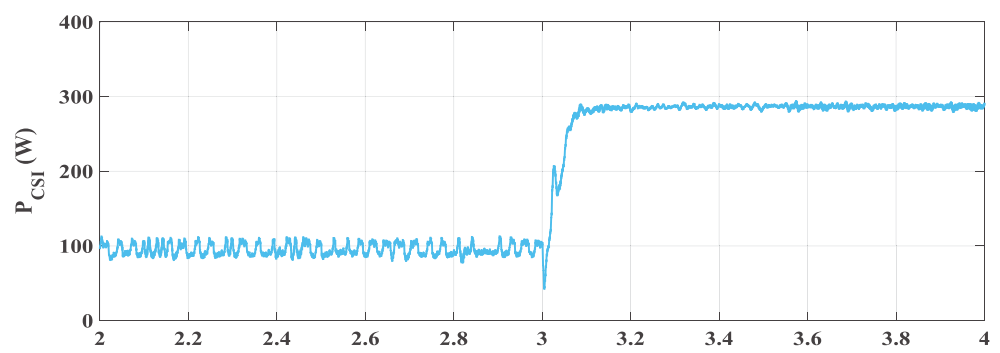

(a)

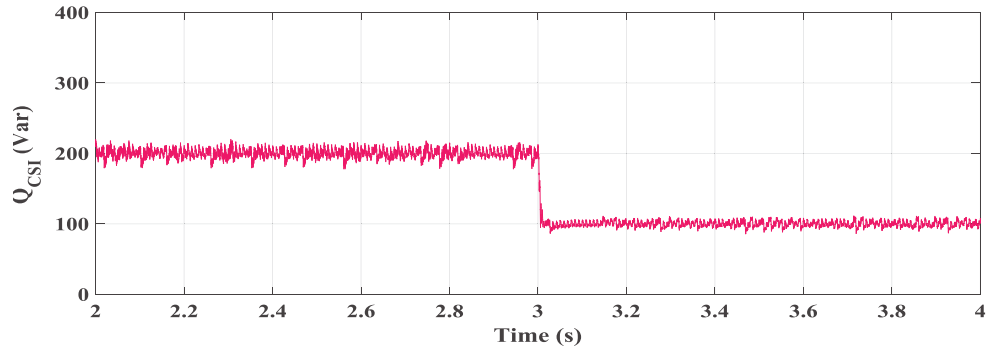

(b)

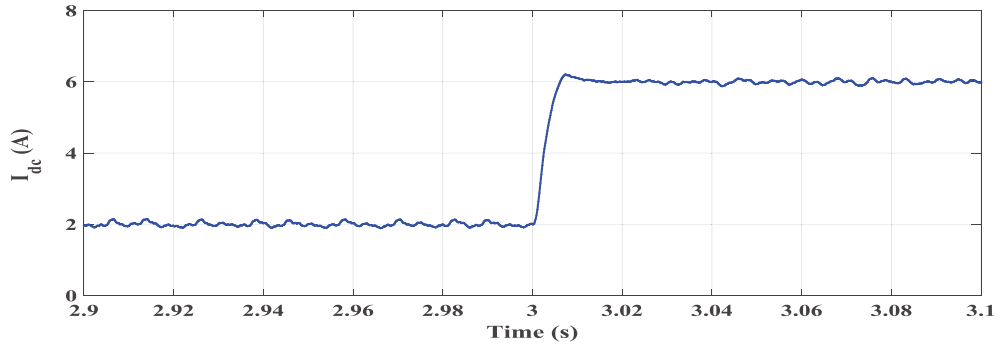

(c)
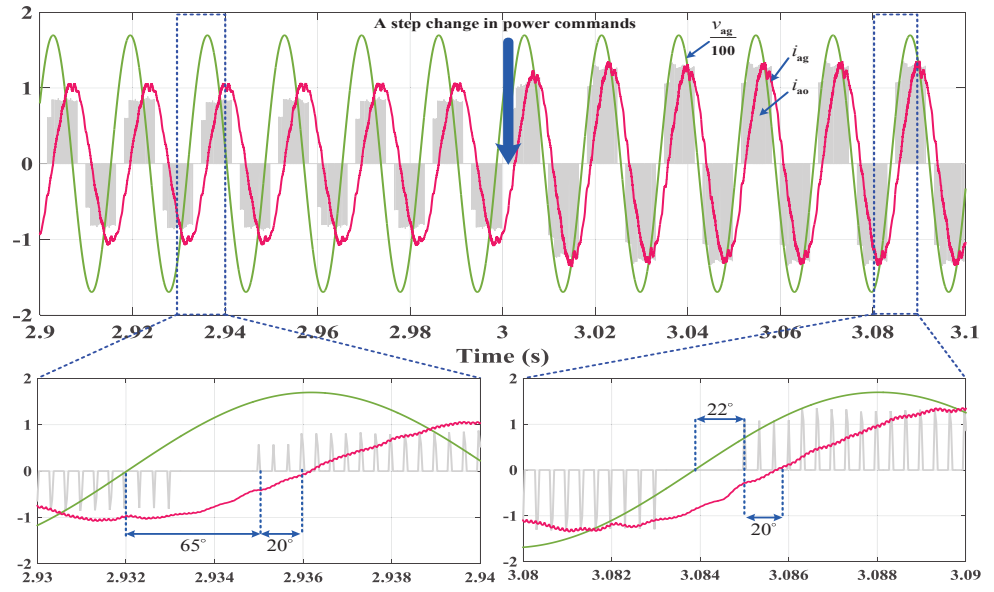

(d)

Figure 5.14: Performance of the proposed method in tracking active and reactive power changes for scenario II. (a) active power step-up. (b) reactive power step-down. (c) dclink current. (d) voltage and current waveforms. 
a promising tracking response to regulate the active and reactive power with almost zero coupling effect. Similarly, it is expected that for second scenario $m_{i}$ is slightly changed from 0.43 to 0.21 in order to reduce the delivered active power to the grid, while $\alpha_{0}$ is adjusted accordingly to increase reactive power, changing from $0.61 \mathrm{rad}\left(35^{0}\right)$ to -0.122 $\operatorname{rad}\left(-7^{0}\right)$.

\subsubsection{Experimental Results}

Figure 5.15 and Figure 5.16 show the performance of power regulation schemes subsequent to step changes in the reference value of the active and reactive power. Description of plots in these figures are the same as in Figure 5.13 and Figure 5.14. However, upon each step change, the dc-link current, active and reactive power commands change to track the reference value changes in almost $20 \mathrm{~ms}$ with a zero steady-state error.

\section{Decoupling Verification of Proposed Control System}

In order to demonstrate the decoupling capability of the proposed control system, two scenarios were considered based on the falls and jumps in the CSI's active and reactive power commands: 1) a step-down and a step-up in the active power reference value

$\left(P_{C S I}^{*}\right)$, while the reactive power command was kept constant and 2) a step-up and a stepdown in the reactive power command $\left(Q_{C S I}^{*}\right)$, while the active power reference value was kept constant.

In first scenario, the performance of both control regulation schemes is experimentally evaluated for two step changes in the reference value of the CSI's active power, as shown in Figure 5.17. Hence, in this scenario, the active power was first assumed to have a step-down from $300 \mathrm{~W}$ (the rated value) to about $100 \mathrm{~W}$, and then after $0.2 \mathrm{~s}$, it had a step-up to $300 \mathrm{~W}$, whereas the reference value for the reactive power was kept constant at $Q_{C S I}^{*}=0$ during the whole process. As can be seen in Figure 5.17(a), corresponding 


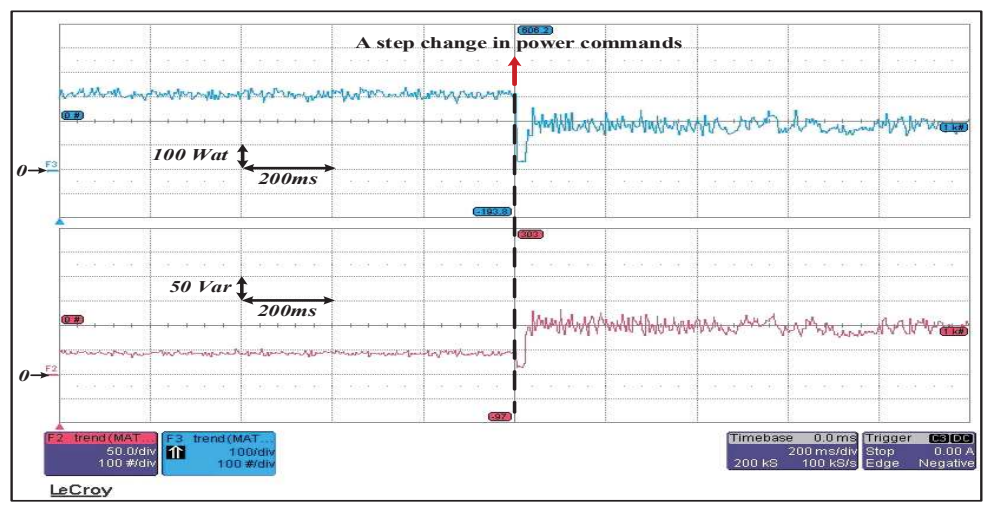

(a)

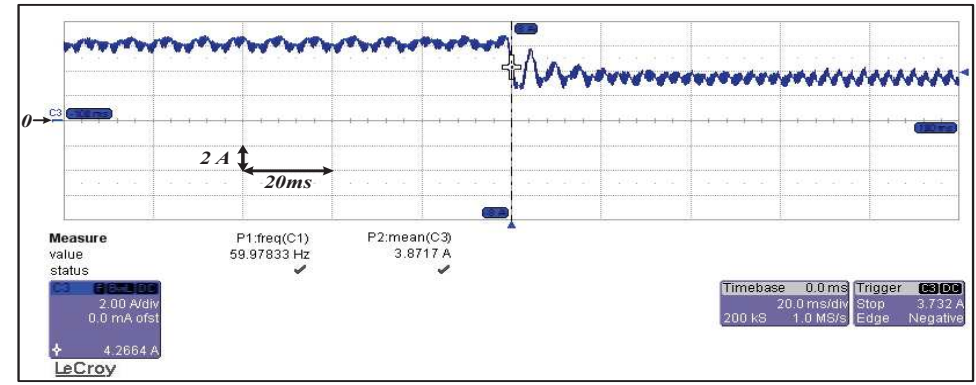

(b)

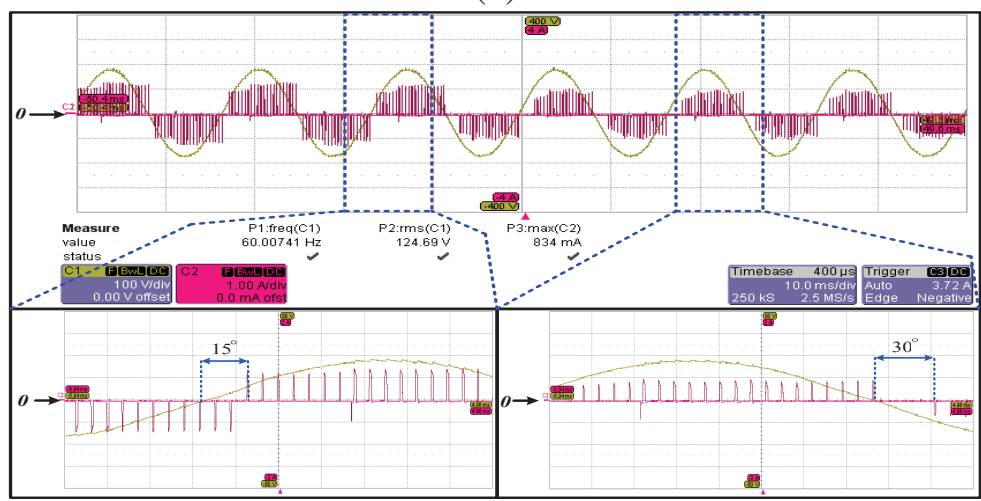

(c)

Figure 5.15: Experimental performance of the proposed method in tracking active and reactive power changes for scenario I. (a) active power step-down. (b) reactive power step-up. (C) dc-link current. (d) voltage and current waveforms.

to the conventional PI controller, the reactive power reference signal experiences the nonnegligible transients upon each step change. The transients verify that the $d$ and $q$ axes are not fully decoupled when the conventional PI-based control scheme was implemented for the CSI-based MIC system. However, for multivariable PI-based controller, the reference 


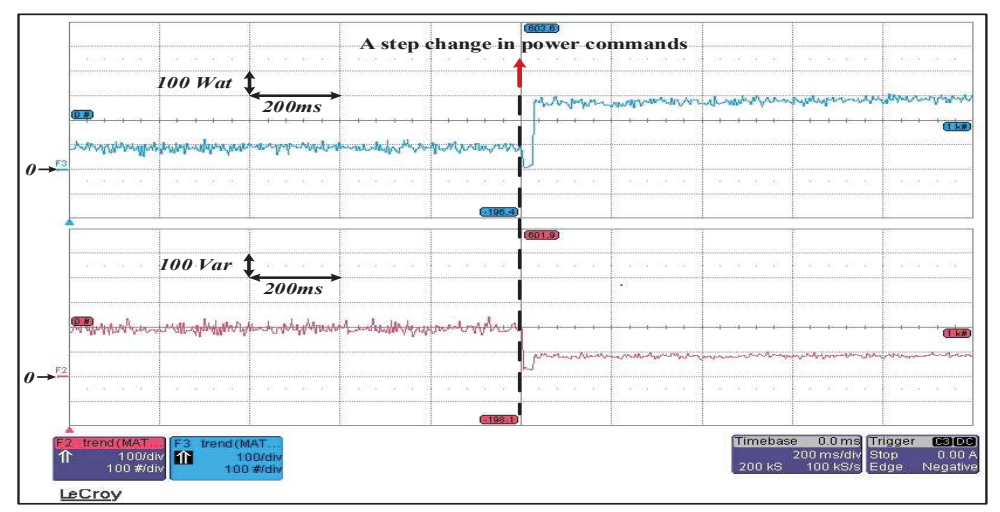

(a)

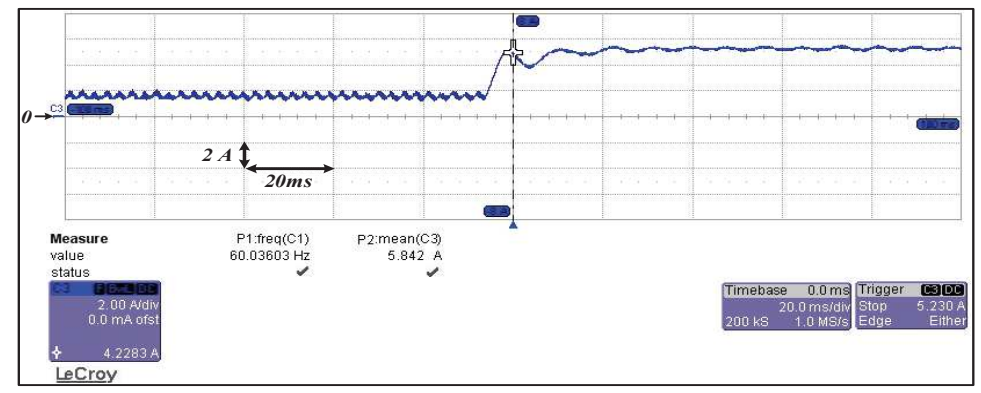

(b)

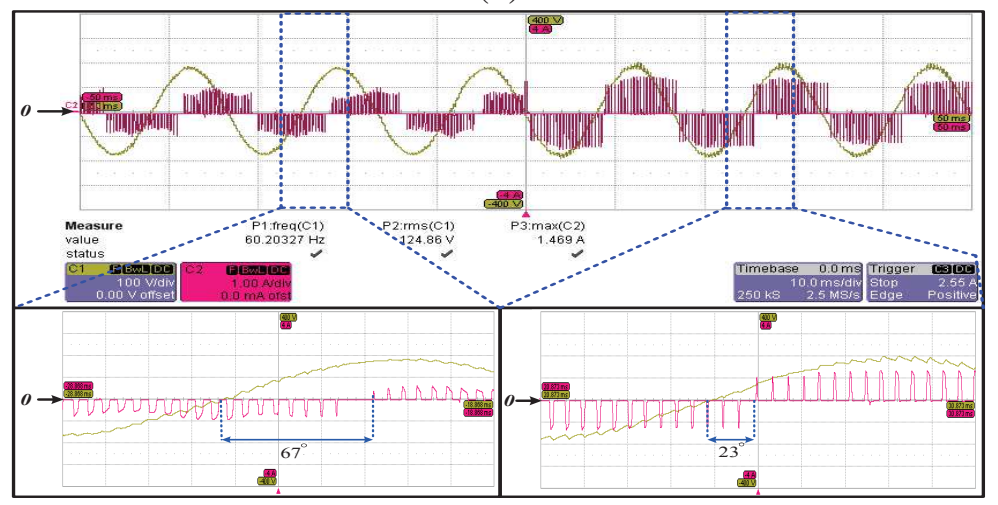

(c)

Figure 5.16: Experimental performance of the proposed method in tracking active and reactive power changes for scenario II. (a) active power step-up. (b) reactive power stepdown. (C) dc-link current. (d) voltage and current waveforms.

value of the reactive power sustains a much less amount of transients, subsequent to each step change in the active power command, as shown in Figure 5.17(b).

Figures 5.17(a) and 5.17(b) clarify that the performance of the multivariable PI-based controller in terms of tracking the step changes in the active power reference value is 


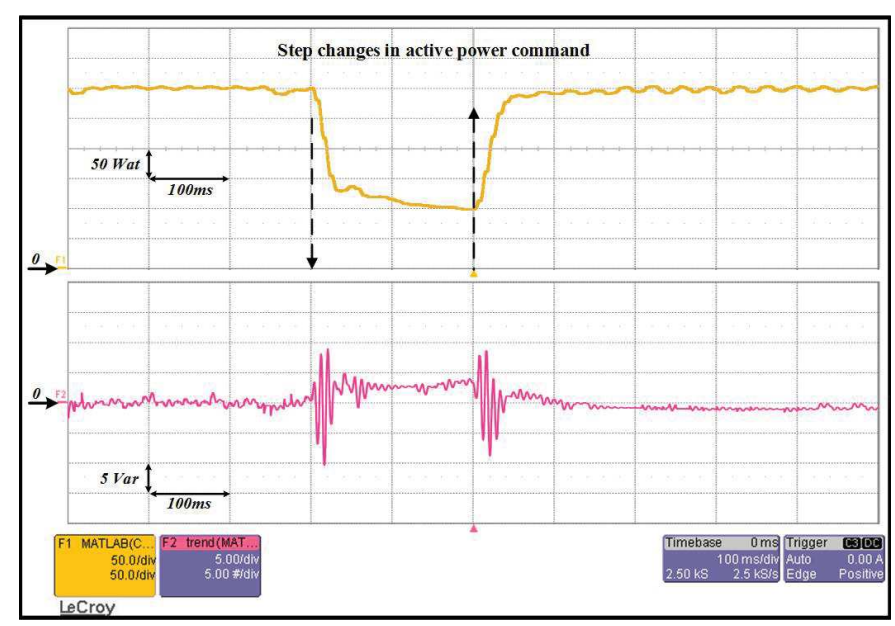

(a)

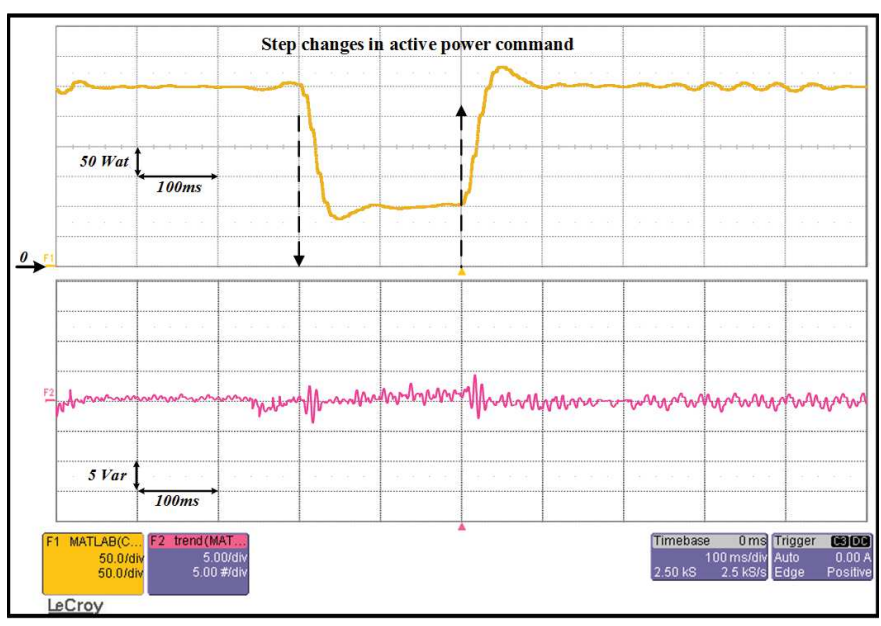

(b)

Figure 5.17: Experimental results for the transient response during step changes in active power command $P_{C S I}^{*}$. (a) Conventional PI-based controller. (b) Multivariable PI-based controller.

almost comparable to that of the conventional PI-based controller. However, contrary to the conventional controller, upon the step changes in the active power command, the proposed multivariable PI controller produces much less transients on the $Q_{C S I}^{*}$ signal.

As demonstrated in overall control block diagram, shown in Figure 5.11, the active and reactive power exchanged between the PV MIC and the grid are obtained through controlling the modulation index $m_{i}$ and the modulation angle $\alpha_{0}$. Thus, for this scenario in order to regulate the active and reactive power independently, the value of the $m_{i}$ was 


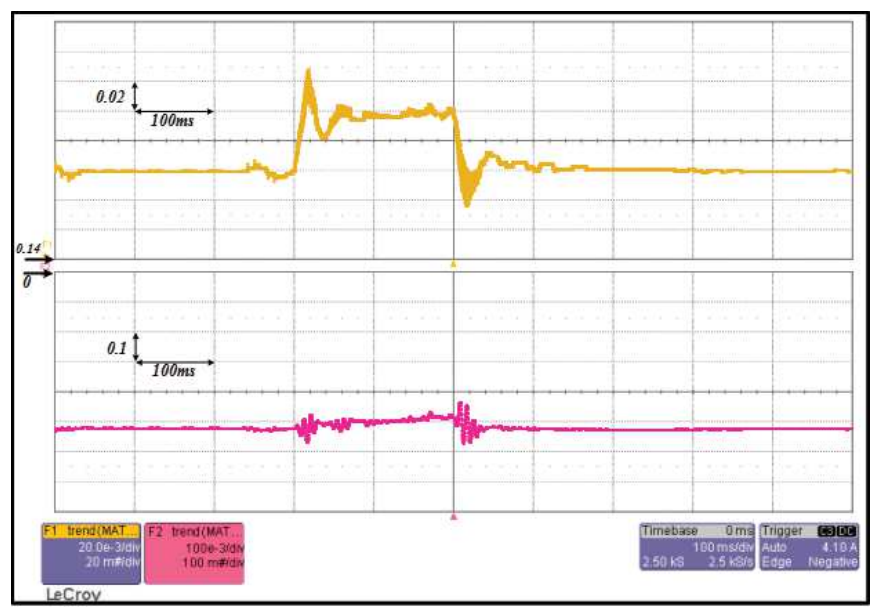

Figure 5.18: Experimental results for the transient response of multivariable PI controller during step changes in active power command. Description of plots: modulation index $m_{i}$ on top and modulation angle $\alpha_{0}$ on bottom.

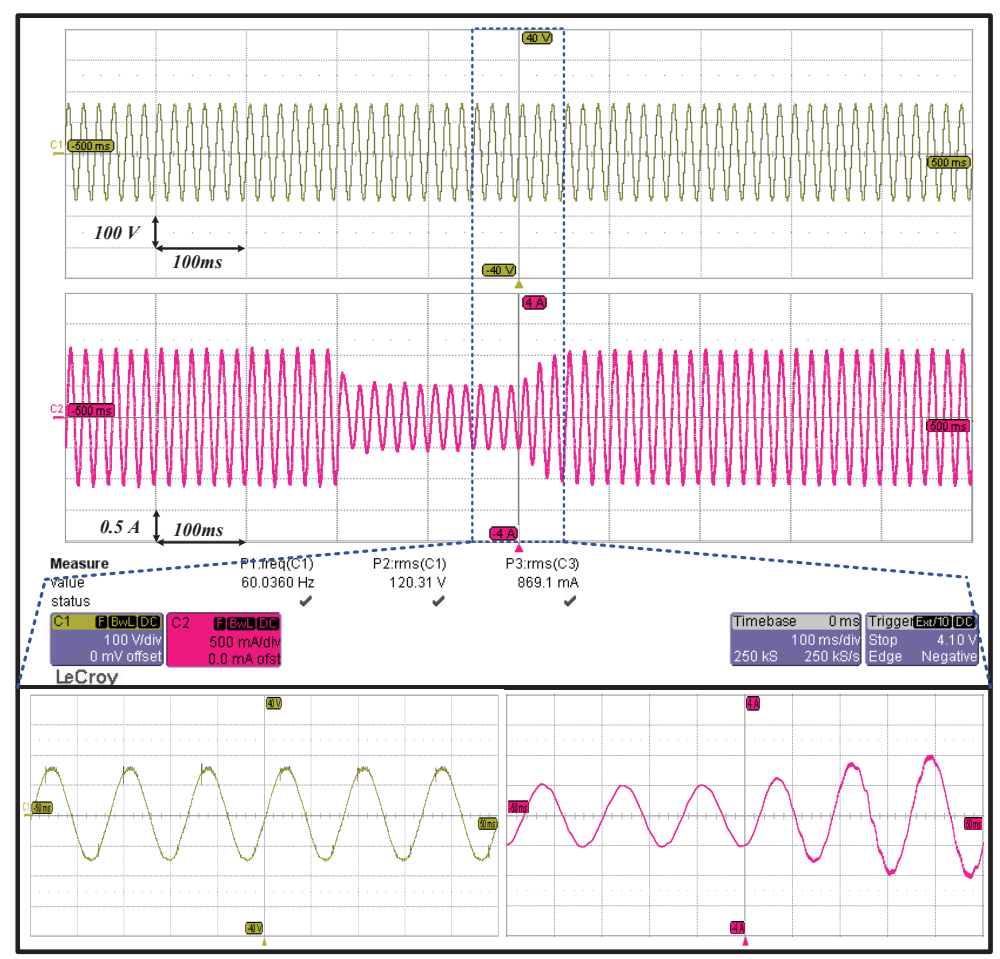

Figure 5.19: Experimental results for the transient response of multivariable PI controller during step changes in active power command. Description of plots: phase-a waveform of the grid voltage on top and grid current on bottom. 


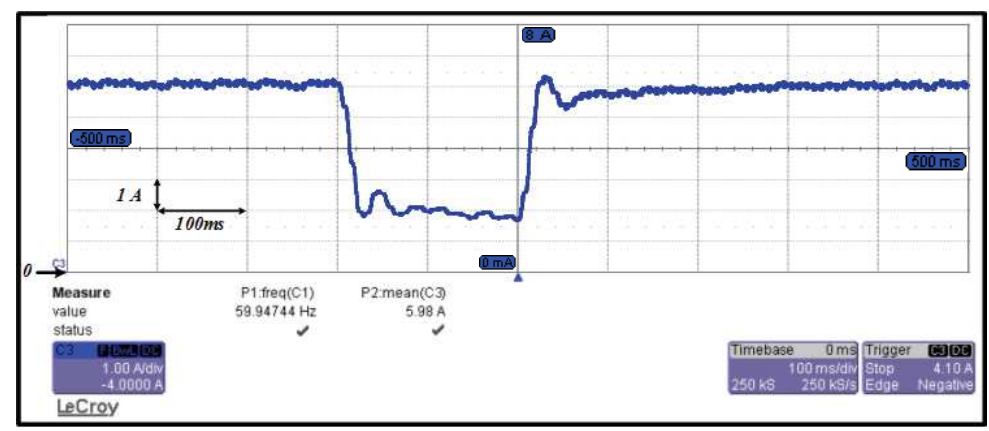

Figure 5.20: Experimental results for the transient response of dc-link current during step changes in active power command.

changed from 0.2 to 0.22 in the step-down mode and returned to the value of 0.2 in the step-up mode, while the value of $\alpha_{0}$ was kept around its initial value of $-0.52 \mathrm{rad}$ by the controller, as shown in Figure 6.19. Based on (5.31), it should be note that there is a phase shift of $\pi / 6 \mathrm{rad}$ between the CSI current phase angle $\varphi_{i n v}$ and modulation angle $\alpha_{0}$, which can be obtained from the $\varphi_{i n v}=\arctan \left(Q_{C S I}^{*} / P_{C S I}^{*}\right)$ and $\alpha_{0}=\varphi_{i n v}-\pi / 6$, respectively.

The phase-a waveform of the grid voltage $v_{a g}$, and the injected grid current $i_{a g}$, are shown in Figure 5.19. As can be seen, the voltage remains unchanged during the step changes. However, upon each step change, the magnitude and phase angle of the grid current change to track the reference value with a zero steady-state error, as shown in Figure 5.19. As was mentioned in the previous section, the filter capacitor is selected to inject $25 \%$ of rated power (around 80 Var), therefore creating a fixed shift angle in the grid current $i_{a g}$ with respect to the $v_{a g}$. As displayed in Figure 5.20, the dc current is decreased from around $6 \mathrm{~A}$ to $2 \mathrm{~A}$ due to the step change of the CSI active power command. It is observed that the proposed multivariable PI controller has a promising tracking response to regulate the active and reactive power.

In second scenario, in order to evaluate the performance of the multivariable PI controller in terms of decoupling the axes during the step changes in the reactive power 


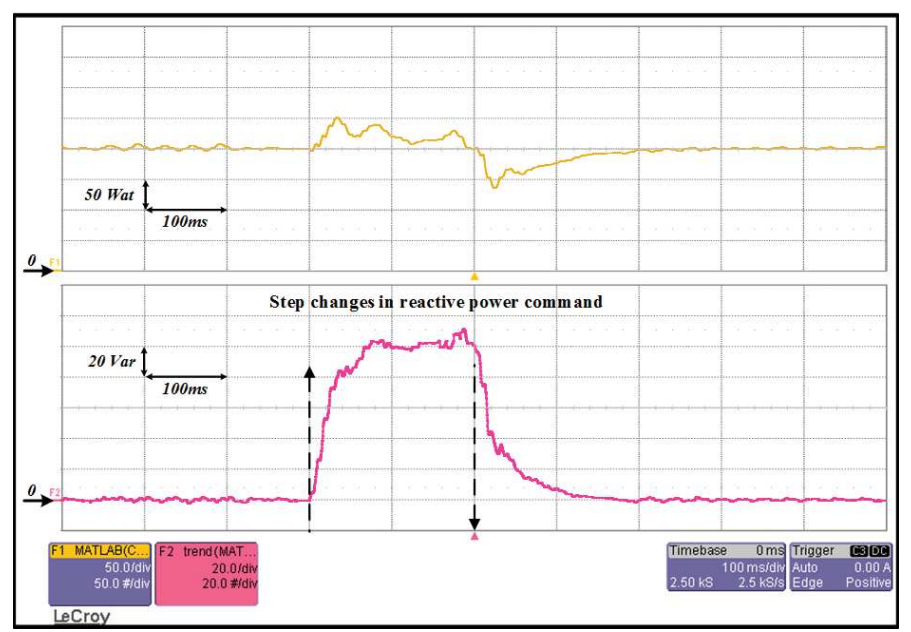

(a)

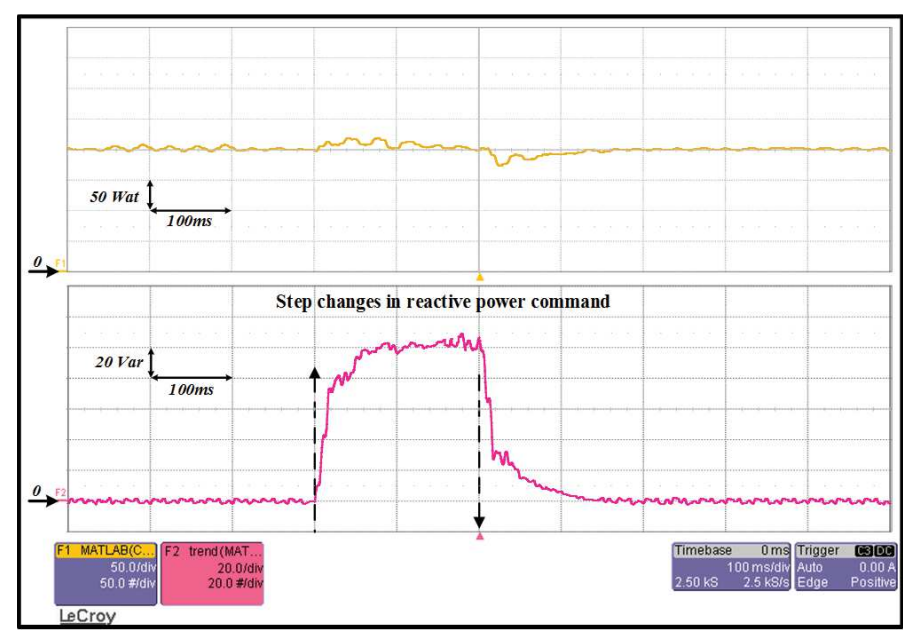

(b)

Figure 5.21: Experimental results for the transient response during step changes in reactive power command $Q_{C S I}^{*}$. (a) Conventional PI-based controller. (b) Multivariable PI-based controller.

command, a similar test to that of the previous scenario was carried out. In this scenario, the reference value for the active power was kept constant at $P_{C S I}^{*}=200 \mathrm{~W}$ during the whole process, whereas the reactive power command first jumped from 0 Var to around 100 Var, then after $200 \mathrm{~ms}$ it was set back to 0 Var, as shown in Figure 5.21.

Comparing Figure 5.21(a) and 5.21(b) again demonstrates that the dynamic performance of the multivariable PI-based controller in terms of tracking the step changes in the reactive power command is comparable to that of the conventional PI-based con- 


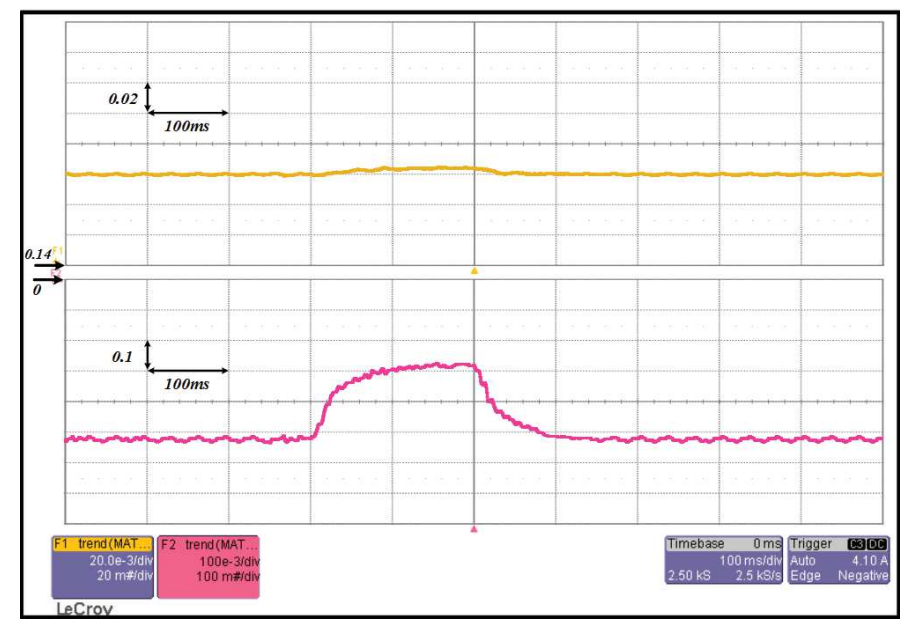

Figure 5.22: Experimental results for the transient response of multivariable PI controller during step changes in reactive power command. Description of plots: modulation index $m_{i}$ on top and modulation angle $\alpha_{0}$ on bottom.

troller. However, the proposed multivariable PI controller generates less transients on the $P_{C S I}^{*}$ signal, upon the step changes in the reactive power reference value.

Similarly, as shown in Figure 5.22, it is observed that for second scenario, the value of $m_{i}$ was adjusted around 0.2 by the controller, while $\alpha_{0}$ was regulated accordingly to have a increase from $-0.52 \mathrm{rad}$ to about $-0.3 \mathrm{rad}$ in order to keep $P_{C S I}^{*}$ constant and to increase the delivered reactive power to the grid during the step changes.

Figure 5.23 (top plot) depicts the phase-a waveform of the grid voltage $v_{a g}$, which are dictated by the grid. Subsequent to each change in the reactive power reference value, the controller regulates the grid current at the desired level, as shown in Figure 5.23 (bottom plot). As shown in Figure 5.24, the multivariable PI controller kept the dc-link current at a constant value of $4 \mathrm{~A}$, corresponding to the active power $200 \mathrm{~W}$, due to the step-up change of the CSI reactive power command. As can be seen, the proposed controller has shown a promising tracking response to regulate the active and reactive powers. 


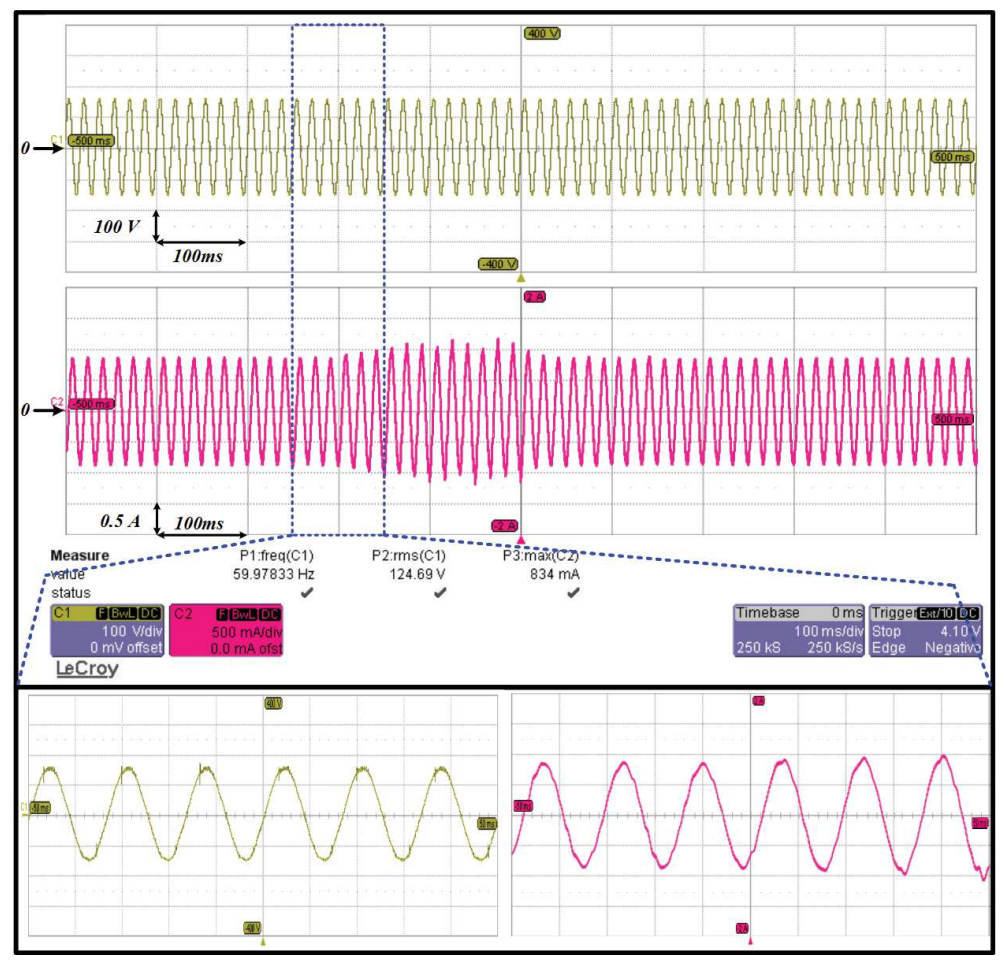

Figure 5.23: Experimental results for the transient response of multivariable PI controller during step changes in reactive power command. Description of plots: phase-a waveform of the grid voltage on top and grid current on bottom.

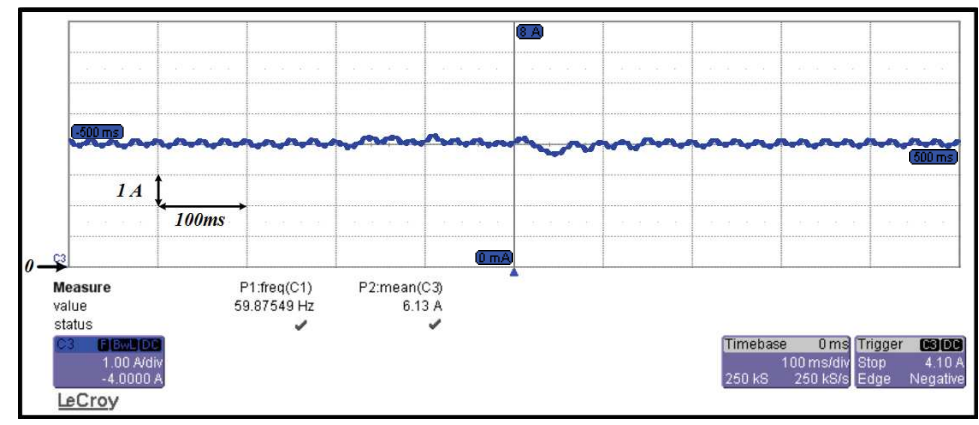

Figure 5.24: Experimental results for the transient response of dc-link current during step changes in reactive power command.

\subsection{Summary}

In this chapter, a 300-VA three-phase single-stage CSI was proposed and experimentally implemented as a testbed for grid-tied PV MIC systems. A multivariable PI-based current control strategy with structural simplicity was presented to independently control the 
active and reactive powers, through regulating the control parameters of $m_{i}$ and $\alpha_{0}$ introduced in the PPWM switching pattern. The experimental results show that the proposed control system has a promising dynamic response in terms of tracking the step changes in the active and reactive power reference values. Furthermore, the multivariable PI control method represents a superior axis decoupling capability, so that the step changes in one power command signal negligibly affect the other. The dynamic behaviors of the threephase grid-tied CSI-based MIC system were studied by using the open-loop eigenvalue analysis of the small signal model. Surprisingly, it is concluded that the dc-link voltage has never affected the stability of the proposed system. The results also reveal that the proposed system stays stable as control parameters of $m_{i}$ and $\alpha_{0}$ vary within the CSI's operating limits.

Considering further expansion of the three-phase PV MIC system, it would be required for MIC to be equipped with the real-time active and reactive power control capability to fulfill the upcoming grid requirements. Thus, these investigations can pave the path for the CSI-based MIC applications into photovoltaic energy generation systems. This study has also presented the tools for further exploring the CSI-based MIC capabilities for other case studies such as stability analysis of closed-loop control systems and large-scale grid-tied PV applications. 


\section{CHAPTER 6}

\section{EXPLORING RELIABLE STRATEGIES FOR PROTECTING SMART GRID UNDER MULTI-FAILURE EVENTS}

\subsection{Overview}

The main contribution of this chapter is to introduce and develop a new game-theoretic framework to analyze how a power grid can dynamically survive concurrent severe multifailure events. In this study, instead of exploring the critical multiple contingencies by creating the random failures via simulation approaches or optimization algorithms, the multiple failures are generated in the game-theoretic framework, as the worst-case scenarios. More importantly, such events with low probability of occurrence, which might cause serious damage to the power system, are considered in the proposed method. Accordingly, this approach provides reasonable decisions for power grid operators about how to allocate the limited budgets on components of the grid network in order to reduce the probability of a successful failure and maximize the power system survivability.

Thus, this work is first accomplished by utilizing a developed optimal load shedding technology in order to calculate the amount of shed load under different types of failures, which represents the physical impacts of the failures on the power grid. Using these quantified physical impacts, a stochastic game for continual dynamic interactions between the failure maker, which generates contingencies with the low and high probability of occurrence, and the power grid operator, is formulated. Then, this chapter proposes a novel learning algorithm to obtain the operator's Nash equilibrium strategies that provide insightful guidelines on how to deploy a limited budget for protecting critical components of the power grid. Simulation results using the IEEE standard 14-bus and IEEE 39-bus benchmark are presented to illustrate the feasibility of the proposed approach. 
The rest of this chapter is organized as follows. Section 6.2 presents the system model and problem formulation. The stochastic game framework is described and analyzed in Section 6.3. The two algorithms to obtain Nash equilibrium strategies for power system operators are presented in Section 6.4, while some illustrative examples are given in Section 6.5. The conclusions are drawn in Section 6.6.

\subsection{System Model and Problem Formulation}

Consider a power system composed of $N_{\mathcal{V}}$ buses (including $N_{g}$ generation buses and $N_{l}$ load buses) and $N_{\mathcal{E}}$ transmission lines. This system can be modeled using a graph $\mathcal{G}:=$ $(\mathcal{V}, \mathcal{E})$ with $N_{\mathcal{V}}$ vertices and $N_{\mathcal{E}}$ edges. The set of vertices $\mathcal{V}=\left\{v_{1}, v_{2}, \ldots, v_{N_{\mathcal{V}}}\right\}$ contains $N_{\mathcal{V}}$ nodes in the graph that include generation plants, transformers, substation devices, and customers. The set $\mathcal{E}=\left\{e_{1}, e_{2}, \ldots, e_{N_{\mathcal{E}}}\right\}$ of edges includes $N_{\mathcal{E}}$ edges corresponding to transmission lines. Hence, the total number of system components is $N_{\mathcal{G}}=N_{\mathcal{V}}+N_{\mathcal{E}}$, which must be protected from failures.

In this section, it is assumed that failure makers can generate the sever failures on the system by distributing their limited budget $B_{f}$ over one or more components of the graph in order to maximize the physical impact of the failures on the system. In contrast, operators need to decide how to allocate their limited budget $B_{d}$ for the protection of the $N_{G}$ components in order to maximize system survivability under such severe failures. Based on the operator decision, the terms of budgets can be the amount of money which needs to spend to reinforce the power system infrastructure, the number of protection and measuring devices installed on critical transmission lines, the number of the technician which should be sent to failed transmission line in order to fix them quickly.

The possible failure events that can be considered in this study are categorized into two groups: a single-failure event, which can affect only one component of the graph at a time, and a multi-failure event, which can simultaneously cut off two or more components 
of the graph. In the literature, the physical impact of a failure event on the system is measured by the cost of shed load following the failure of components. As mentioned in [175], the optimal load shedding problem can be formulated to minimize the cost of load, which must be shed considering power system stability constraints, as follows

$$
\begin{gathered}
\min _{\mathbf{z}, \boldsymbol{\theta}} \sum_{j=1}^{N_{l}} w_{l j} u_{l j} z_{l j}, \\
\text { s.t. } \quad \boldsymbol{\Gamma}^{T} \boldsymbol{B} \sin (\boldsymbol{\Gamma} \boldsymbol{\theta})-(\boldsymbol{p}+\boldsymbol{z})=0, \\
\mathbf{0} \leq \boldsymbol{p}_{g}+\boldsymbol{z}_{g} \leq \boldsymbol{p}_{g}, \\
\boldsymbol{p}_{l} \leq \boldsymbol{p}_{l}+\boldsymbol{z}_{l} \leq \mathbf{0}, \\
\boldsymbol{\theta}_{\min } \leq \boldsymbol{\Gamma} \boldsymbol{\theta} \leq \boldsymbol{\theta}_{\max }, \\
\boldsymbol{c}_{\min } \leq \boldsymbol{B} \sin (\boldsymbol{\Gamma} \boldsymbol{\theta}) \leq \boldsymbol{c}_{\max },
\end{gathered}
$$

where $\boldsymbol{w}_{l}=\left[w_{l 1}, w_{l 2}, \ldots, w_{l N_{l}}\right]^{T}$ is the weight vector introduced to represent the relative importance of different kinds of load buses. $\boldsymbol{u}_{l}=\left[u_{l 1}, u_{l 2}, \ldots, u_{l N_{l}}\right]^{T}$ represents the load cost vector, each of those components explains independent load values. $\boldsymbol{p}=\left[\boldsymbol{p}_{g}^{T}, \boldsymbol{p}_{l}^{T}\right]^{T}$ represents the power distribution over $N_{\mathcal{V}}$ buses, in which $\boldsymbol{p}_{g}=\left[p_{g 1}, p_{g 2}, \ldots, p_{g N_{g}}\right]^{T} \geq \mathbf{0}$ refers to power generation over $N_{g}$ generation buses, while $\boldsymbol{p}_{l}=\left[p_{l 1}, p_{l 2}, \ldots, p_{l N_{l}}\right]^{T} \leq \mathbf{0}$ refers to load distribution over $N_{l}$ load buses. $\boldsymbol{z}=\left[\boldsymbol{z}_{g}^{T}, \boldsymbol{z}_{l}^{T}\right]^{T}$ represents the changes in power assignment for $N_{\mathcal{V}}$ buses due to component failures and corresponding load shedding, in which $\boldsymbol{z}_{g}=\left[z_{g 1}, z_{g 2}, \ldots, z_{g N_{g}}\right]^{T} \leq \mathbf{0}$ refers to power reduction at given generation buses while $\boldsymbol{z}_{l}=\left[z_{l 1}, z_{l 2}, \ldots, z_{l N_{l}}\right]^{T} \geq \mathbf{0}$ refers to load to be shed. $\boldsymbol{\theta}=$ $\left[\theta_{1}, \theta_{2}, \ldots, \theta_{N_{\mathcal{V}}}\right]^{T}$ gives the phase angle at each bus, while $\boldsymbol{\theta}_{\min }$ and $\boldsymbol{\theta}_{\max }$ are, respectively, the minimized and maximized limitations of the phase angle at each bus. $\Gamma \in R^{N_{\mathcal{V}} \times N_{\mathcal{E}}}$ is 
the incidence matrix for the topology of the power grid, and $\boldsymbol{B} \in R^{N_{\mathcal{E}} \times N_{\mathcal{E}}}$ is a diagonal matrix, in which those diagonal entries correspond to line admittances. $\boldsymbol{c}_{\min }$ and $\boldsymbol{c}_{\max }$ independently represent the minimized and maximized power limitations of each branch.

In this considered problem, constraint (6.2) corresponds to the physical power flow equation that must be satisfied during the load shedding. Constraint (6.3) ensures that, for $N_{g}$ generation buses, the power can only be reduced without being added. Similarly, in (6.4), $\boldsymbol{p}_{l} \leq \boldsymbol{p}_{l}+\boldsymbol{z}_{l}$ guarantees that the load is being shed, not being added, while $\boldsymbol{p}_{l}+\boldsymbol{z}_{l} \leq \mathbf{0}$ guarantees that the amount of shed load is not so much that may turn a load into generation. Constraint (6.5) indicates that, in order to keep the power system in a stable state, each phase angle of $N_{\mathcal{V}}$ buses should be in such an interval. Similarly, the capacity limits of transmission lines are given in the constraint (6.6).

Constraints (6.2) and (6.6) including trigonometric sine functions render the load shedding problem a hard non-convex optimization problem. However, as described in [175], the phase angle differences $\boldsymbol{\Gamma} \boldsymbol{\theta} \approx \mathbf{0}$ under normal operations of the power grid. If the phase angle vector is limited to $\boldsymbol{\theta}_{\min }=-\pi / 2$ and $\boldsymbol{\theta}_{\max }=\pi / 2$, the non-convex optimization problem can be linearized as a linear programming problem, shown according to $\sin (\boldsymbol{\Gamma} \boldsymbol{\theta}) \approx \boldsymbol{\Gamma} \boldsymbol{\theta}$ for $\boldsymbol{\Gamma} \boldsymbol{\theta} \approx 0$. Thus, (5.1) can be solved by efficient techniques, including simplex methods [176] and interior-point methods [177] for optimal solutions. A severe multiple failure as the worst case scenario could lead to a combination of components failures, so that the incidence matrix $\Gamma$ must be updated according to the effected component in the system. Taking the updated incidence matrix $\Gamma$ as an input parameter, the cost of shed load under the multiple failures will then be equal to $\sum_{j=1}^{N_{l}} w_{l j} u_{l j} z_{l j}$ derived by (6.1). Using this optimal load shedding technique, a game-theoretic approach for analyzing the continual interactions between the failure maker and the power grid operator is proposed in the next section. 


\subsection{Stochastic Game-theoretic Approach}

In this section, a stochastic game is formulated in the normal form $\Pi=\left\langle\mathcal{S}, \mathcal{F}, \mathcal{D}, \mathbf{R}^{\mathcal{F}}, \mathbf{R}^{\mathcal{D}}, \mathbf{T}\right\rangle$, in which players of the game are the failure maker (player 1), representing the nature of the power system, and the power grid operator (player 2). The key components of the game include [178]:

$\mathcal{S}:=\left\{s_{1}, s_{2}, \ldots, s_{N_{\mathcal{S}}}\right\}$ represents the power grid's state space.

$\mathcal{F}:=\left\{f_{1}, f_{2}, \ldots, f_{N_{\mathcal{F}}}\right\}$ represents the failure maker's action space, which contains all possible methods of allocating budget $B_{f}$ over the $N_{\mathcal{G}}$ components of the graph.

$\mathcal{D}:=\left\{d_{1}, d_{2}, \ldots, d_{N_{\mathcal{D}}}\right\}$ represents the operator's response space, which contains all possible methods to distribute budget $B_{d}$ over the $N_{\mathcal{G}}$ components of the graph.

$\mathbf{R}^{\mathcal{F}}(s):=\left[R_{f, d}^{\mathcal{F}}(s)\right]_{N_{\mathcal{F}} \times N_{\mathcal{D}}}$ is the failure maker's expected reward matrix with respect to failure event $f \in \mathcal{F}$ and the operator's response $d \in \mathcal{D}$.

$\mathbf{R}^{\mathcal{D}}(s):=\left[R_{f, d}^{\mathcal{D}}(s)\right]_{N_{\mathcal{F}} \times N_{\mathcal{D}}}$ is the operator's expected reward matrix corresponding to the operator's response $d \in \mathcal{D}$ and the failure event $f \in \mathcal{F}$.

$\mathbf{T}(f, d)=\left[T_{s_{i}, s_{j}}(f, d)\right]_{N_{\mathcal{F}} \times N_{\mathcal{D}}}$ is the power system's state transition matrix corresponding to the failure event $f \in \mathcal{F}$ and the operator's response $d \in \mathcal{D}$.

Let $\rho^{\mathcal{S}}[t]$ be the probability distribution on the power system's state space at time $t$, then the probability distribution at time $t+1$ will be $p^{\mathcal{S}}[t+1]=\mathbf{T}(f, d) \times \rho^{\mathcal{S}}[t]$. The matrix entry $T_{s_{i}, s_{j}}(f, d)$ indicates the probability that state $s_{i}$ transitions to state $s_{j}$ under a failure action $f$ and an operator's response $d$. The $T_{s_{i}, s_{j}}(f, d)$ can be derived based on two probabilities, the failure probability $p_{i}^{\text {fail }}(f, d)$ that the normal component $i$ of the power grid fails in one time step under a failure action $f$ and an operator's response $d$ and the recover probability $p_{i}^{\text {rec }}(f, d)$ that the failed component $i$ of the power grid recovers in one time step under a failure action $f$ and an operator's response $d$. Assume the $p_{i}^{\text {fail }}(f, d)$ and $p_{i}^{\text {rec }}(f, d)$ depend on the operator's budgets and the failure maker's budgets deployed on 
each component, then $p_{i}^{\text {fail }}(f, d)$ and $p_{i}^{\text {rec }}(f, d)$ can be formulated, as follows

$$
\left\{\begin{array}{l}
p_{i}^{\text {fail }}(f, d)=\left[c_{i}^{f} /\left(1+c_{i}^{f}\right)\right] \times\left[c_{i}^{f} /\left(1+c_{i}^{d}\right)\right], \\
p_{i}^{\text {rec }}(f, d)=\left[c_{i}^{d} /\left(1+c_{i}^{d}\right)\right] \times\left[1 /\left(1+c_{i}^{d}\right)\right],
\end{array}\right.
$$

where $c_{i}^{f(d)}$ is the allocated budget of failures (response mechanisms) related to action $f(d)$ that happened at the component $i$ of the system.

As it is mentioned in the previous section, for the power grid composed of $N_{\mathcal{G}}$ components, the failure makers and operators are constrained by a finite amount of budget. Thus, the budget $B_{f}$ for multiple failures and budget $B_{d}$ for the operator's response mechanisms are implemented at a given time.

Here, it is defined that each failure $f_{i} \in \mathcal{F}, i=1, \ldots, N_{\mathcal{F}}$, as a strategy of allocating possible budgets over $N_{\mathcal{G}}$ components

$$
\left\{\begin{array}{l}
f_{i}=\left[c_{i, 1}^{f}, c_{i, 2}^{f}, \ldots, c_{i, N_{\mathcal{G}}}^{f}\right], \\
\sum_{j=1}^{N_{\mathcal{G}}} c_{i, j}^{f}=B_{f}
\end{array}\right.
$$

where $0 \leq c_{i, j}^{f} \leq B_{f}, j=1,2 \ldots, N_{\mathcal{G}}$, represents the allocated budget for failures, which happened for component $j$ of the grid. Similarly, each operator's response $d_{i} \in \mathcal{D}, i=$ $1, \ldots, N_{\mathcal{D}}$, conditions one strategy to distribute its limited budget over $N_{\mathcal{G}}$ elements

$$
\left\{\begin{array}{l}
d_{i}=\left[c_{i, 1}^{d}, c_{i, 2}^{d}, \ldots, c_{i, N_{\mathcal{G}}}^{d}\right]^{T}, \\
\sum_{j=1}^{N_{\mathcal{G}}} c_{i, j}^{d}=B_{d}
\end{array}\right.
$$

where $0 \leq c_{i, j}^{d} \leq B_{d}, j=1,2 \ldots, N_{\mathcal{G}}$, denotes the allocated budget for protecting the component $j$ of the grid.

\subsubsection{Nash Equilibrium Strategies}

In the proposed stochastic game $\Pi$, for a given state $s \in \mathcal{S}$, a pair action $(f, d)$ will lead to an immediate reward for both sides of the game. Then, the state $s$ transitions to 
the next state $s_{i}, i=1, \ldots, N_{\mathcal{S}}$, based on transition probabilities $T_{s, s_{i}}(f, d)$, satisfying the constraint $\sum_{i=1}^{N_{\mathcal{S}}} T_{s, s_{i}}(f, d)=1$.

For a given state $s \in \mathcal{S}$, a pair action $(f, d)$, and a consequent disruption, the expected reward of the failure maker, denoted by $R_{f, d}^{\mathcal{F}}(s)$, is measured by the expected cost of load that must be shed due to the component failures of the power grid, as follows

$$
R_{f, d}^{\mathcal{F}}(s)=\sum_{i=1}^{N_{\mathcal{S}}}\left(T_{s, s_{i}}(f, d) \times \sum_{i=1}^{N_{l}} w_{l j} u_{l j} z_{l j}\right),
$$

in which $\sum_{i=1}^{N_{l}} w_{l j} u_{l j} z_{l j}$ is derived by the optimal load shedding problem (6.1).

For generating the severe multi-failure events as a worst-case scenario, we intend to maximize the expected reward for the failure maker, while the power grid operator intends to minimize it. Thus, the expected reward for operator is just the negative of the expected reward for the failure maker, presented by $R_{f, d}^{\mathcal{D}}(s)=-R_{f, d}^{\mathcal{F}}(s)$. The proposed stochastic game can be therefore considered as a zero-sum stochastic game [179].

So far, the immediate rewards for both sides of the game at each state game has been specified, but not how these rewards are aggregated into an overall payoff. To solve this problem, the most commonly used aggregation method is the discounted-sum reward [180]. In the proposed game, the discounted-sum reward of the failure maker is the discounted sum of expected rewards at each time step $t$, with a discount factor $\beta \in[0,1)$ :

$$
Q:=\sum_{t=0}^{\infty} \beta^{t} R_{f, d}^{\mathcal{F}}(s(t)) .
$$

where $\beta^{t}$ is the weight of the immediate reward at the time step $t$, representing the relative importance of the immediate reward in the overall payoff (a smaller $\beta$ leads to lower future payoffs).

The discounted-sum reward of the power grid operator is the negative of this number. The goal of the failure maker is to generate the worst multi-failure conditions in order to maximize the long-term reward $Q$, while facing the operator, who intends to minimize it. 
In order to solve the two players' optimal strategies of a stochastic game in the normal form $\Pi$, one popular solution is that of a Nash equilibrium in mixed strategies [181], in which it is a state of the game such that no player can increase its reward by unilaterally deviating from this equilibrium state. For mixed strategies, the strategies for generating severe multi-failure events and corresponding response mechanisms are defined as probability distributions over their space $\mathcal{F}$ and $\mathcal{D}$, respectively. Thus, the mixed strategy of a failure maker for a given state $s[t]$ will be

$$
\left\{\begin{array}{l}
\boldsymbol{\rho}_{\mathcal{F}}(s[t]):=\left[\operatorname{Pr}\left\{f(s[t])=f_{1}\right\}, \ldots, \operatorname{Pr}\left\{f(s[t])=f_{N_{\mathcal{F}}}\right\}\right]^{T}, \\
\sum_{i=1}^{N_{\mathcal{F}}} \operatorname{Pr}\left\{f(s[t])=f_{i}\right\}=1,
\end{array}\right.
$$

where $\operatorname{Pr}\left\{f(s[t])=f_{i}\right\}$ represents the probability of choosing a failure event $f_{i}$ in state $s[t] \in \mathcal{S}$.

Correspondingly, the operator's response mixed strategy in state $s[t]$ is given by:

$$
\left\{\begin{array}{l}
\boldsymbol{\rho}_{\mathcal{D}}(s[t]):=\left[\operatorname{Pr}\left\{d(s[t])=d_{1}\right\}, \ldots, \operatorname{Pr}\left\{d(s[t])=d_{N_{\mathcal{D}}}\right\}\right]^{T}, \\
\sum_{i=1}^{N_{\mathcal{D}}} \operatorname{Pr}\left\{d(s[t])=d_{i}\right\}=1,
\end{array}\right.
$$

where $\operatorname{Pr}\left\{d(s[t])=d_{i}\right\}$ indicates the probability of selecting an operator's response $d_{i}$ in state $s[t] \in \mathcal{S}$. Based on the above equations, the Nash equilibrium can be defined as follows:

\section{Definition 1}

Consider the proposed stochastic game $\Pi=\left\langle\mathcal{S}, \mathcal{F}, \mathcal{D}, \boldsymbol{R}^{\mathcal{F}}, \boldsymbol{R}^{\mathcal{D}}, \boldsymbol{T}\right\rangle$, where rewards $\boldsymbol{R}^{\mathcal{F}}$ and $\boldsymbol{R}^{\mathcal{D}}$ are derived by solving the optimal load shedding problem (1). A Nash equilibrium for time step $t$ is a tuple of two mixed strategies $\left(\boldsymbol{\rho}_{\mathcal{F}}^{*}, \boldsymbol{\rho}_{\mathcal{D}}^{*}\right)$, such that for all failure mixed strategies $\rho(\mathcal{F})$ and the operator's response mixed strategies $\rho(\mathcal{D})$, it satisfies the following set of inequalities:

$$
Q\left(s[t], \boldsymbol{\rho}_{\mathcal{F}}, \boldsymbol{\rho}_{\mathcal{D}}^{*}\right) \leq Q\left(s[t], \boldsymbol{\rho}_{\mathcal{F}}^{*}, \boldsymbol{\rho}_{\mathcal{D}}^{*}\right) \leq Q\left(s[t], \boldsymbol{\rho}_{\mathcal{F}}^{*}, \boldsymbol{\rho}_{\mathcal{D}}\right)
$$


Although the existence of the Nash equilibrium for static games is guaranteed by the Nash's theorem [181], in the case of stochastic games, the possible number of strategies is infinite and is known only in very special cases of stochastic games. However, in this game, we limit our study to stationary optimal strategies by solving the mixed strategies for both sides of the game in each state, instead of each time step, where $\boldsymbol{\rho}_{\mathcal{F}}(s)=\boldsymbol{\rho}_{\mathcal{F}}(s[t])$ and $\boldsymbol{\rho}_{\mathcal{D}}(s)=\boldsymbol{\rho}_{\mathcal{D}}(s[t]), \forall t$ are optimal. For zero-sum discounted stochastic games, it has been proved in [178] that there exists an unique Nash equilibrium in stationary strategies for both sides of the game. Therefore, there always exists a Nash equilibrium for generating the severe multi-failure events and guiding the power grid operator with the stationary strategy selection in the proposed stochastic game $\Pi$.

\subsection{Game Solution}

In this section, the two-player zero-sum stochastic game $\Pi$, as proposed in Section 6.3, is solved. The main objective is to characterize Nash equilibrium strategies for each state $s \in \mathcal{S}$, where Nash equilibrium strategy of the one side of the game is the optimal strategy

to maximize the minimum discounted sum of expected rewards under the opponent's optimal strategy. Due to the zero-sum nature of the game, it is sufficient to describe the solution of optimal strategies for only one side of the game. Therefore, hereinafter, the operator's side of the game is considered, as the solution for the failure maker will be analogous.

Using the minimax Q-learning algorithm presented in [181], the operator's Nash equilibrium strategies can be derived recursively through the following dynamic programming approach. At time step $t$, the optimal discounted sum of expected rewards $Q^{*}$, for a given state $s$ and a pair action $(f, d)$, can be devised iteratively by the following recursions

$$
Q_{f, d}^{t+1}(s)=R_{f, d}^{\mathcal{F}}(s)+\beta \sum_{i=1}^{N_{\mathcal{S}}}\left[T_{s, s_{i}}(f, d)\right] V\left(s_{i}\right)
$$




$$
V\left(s_{i}\right)=\min _{\boldsymbol{\rho}_{\mathcal{D}}} \max _{f} \boldsymbol{\rho}_{\mathcal{D}}^{T}\left(s_{i}\right) Q_{f, d}^{t}\left(s_{i}\right)
$$

for a given initial condition $Q_{0}$. One of the drawbacks of this method is that every iteration of this algorithm just depends on rewards derived at the current time step, while ignoring those derived before steps. Therefore, the computational complexity of such algorithm grows exponentially with the size of the power grid, making it impractical for grids with reasonable sizes. Inspired by the improved linear programming algorithm for Markov decision processes (MDPs) in [179], this study introduces a changeable learning rate $\alpha_{t}=$ $1 /(t+1)^{\omega}$ for each time step $t$, for $\omega \in[0.1,1]$, into the minimax Q-learning algorithm. Using the learning rate $\alpha_{t}$, two new recursions are defined for computing the optimal discounted sum of expected rewards $Q^{*}$ at time step $t$, as follows

$$
\begin{gathered}
Q_{f, d}^{t+1}(s)=\alpha_{t}\left\{R_{f, d}^{\mathcal{F}}(s)+\beta \sum_{i=1}^{N_{\mathcal{S}}}\left[T_{s, s_{i}}(f, d)\right] \times V\left(s_{i}\right)\right\}+\left(1-\alpha_{t}\right) Q_{f, d}^{t}(s) . \\
V\left(s_{i}\right)=\min _{\boldsymbol{\rho}_{\mathcal{D}}} \max _{f} \boldsymbol{\rho}_{\mathcal{D}}^{T}\left(s_{i}\right) Q_{f, d}^{t}\left(s_{i}\right)
\end{gathered}
$$

for a given initial condition $Q_{0}$. Also, (6.18) can be formulated as a linear constrained optimization problem

$$
\left\{\begin{array}{l}
\min _{\boldsymbol{\rho}_{D}} V\left(s_{i}\right), \\
\boldsymbol{\rho}_{\mathcal{D}}^{T}\left(s_{i}\right) Q_{f, d}^{t}\left(s_{i}\right) \leq V\left(s_{i}\right), \forall f \in \mathcal{F},
\end{array}\right.
$$

The operator's mixed strategy $\boldsymbol{\rho}_{\mathcal{D}}^{*}(s), \forall s \in \mathcal{S}$, calculated by (6.19), is the Nash equilibrium strategy. The fixed points of (6.17) and (6.18), $Q^{*}$ and $V^{*}$, lead to the optimal minmax solution for the operator. Correspondingly, the Nash equilibrium strategy for the failure maker $\boldsymbol{\rho}_{\mathcal{F}}^{*}(s) \forall s \in \mathcal{S}$, can be obtained by solving the dual of the linear constraint optimization (6.18)

$$
\left\{\begin{array}{l}
\max _{\boldsymbol{\rho}_{F}} V_{\text {dual }}\left(s_{i}\right), \\
\boldsymbol{\rho}_{\mathcal{F}}^{T}\left(s_{i}\right) Q_{f, d}^{t}\left(s_{i}\right) \geq V_{\text {dual }}\left(s_{i}\right), \forall d \in \mathcal{D},
\end{array}\right.
$$




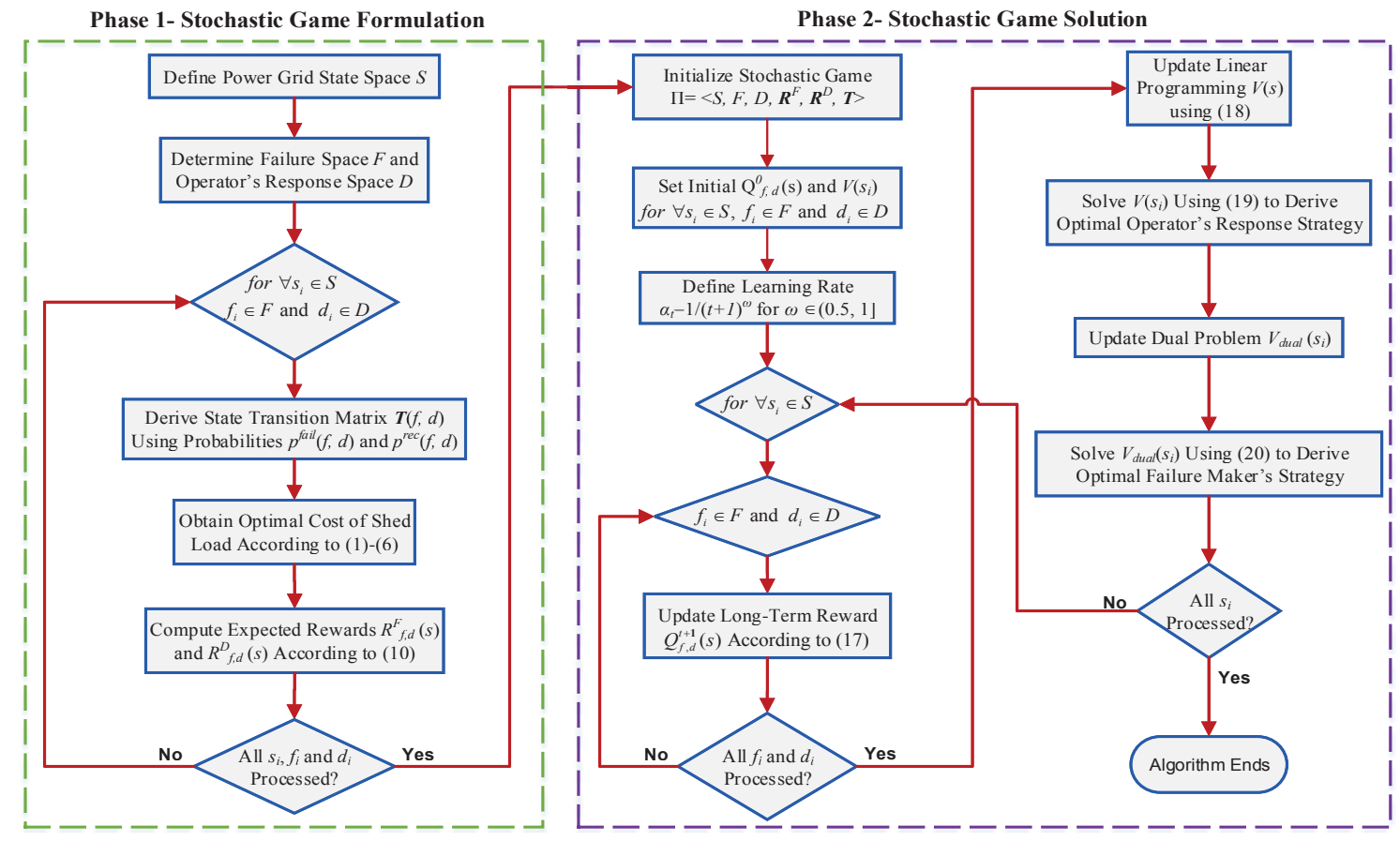

Figure 6.1: Flowchart of proposed stochastic game approach.

For the zero-sum stochastic games, the strong max-min property in [178] proves that the strong duality applies and $V_{\text {dual }}\left(s_{i}\right)$ is equal to $V\left(s_{i}\right)$. Thus, the tuple of Nash equilibrium strategies $\left(\boldsymbol{\rho}_{\mathcal{D}}^{*}(s), \boldsymbol{\rho}_{\mathcal{F}}^{*}(s)\right) \forall s \in \mathcal{S}$, obtained by (6.19) and (6.20), is the Nash equilibrium that we are looking for each state of the power grid. The convergence of the computing procedure of the Nash equilibrium for the proposed stochastic game is proved in [178], so that the reward sequence $\left\{Q_{t}\right\}_{t \rightarrow \infty}$, derived by the algorithm, converges to the optimal point $Q^{*}$. The overall procedure of computing the game rewards and Nash equilibrium of the proposed stochastic game $\Pi$ is depicted in the flowchart, shown in Figure 6.1.

\subsection{Simulation Results and Analysis}

In this section, the performance of the proposed stochastic game algorithm was evaluated using the standard IEEE 14-bus system from MATPOWER, as a benchmark system, to 

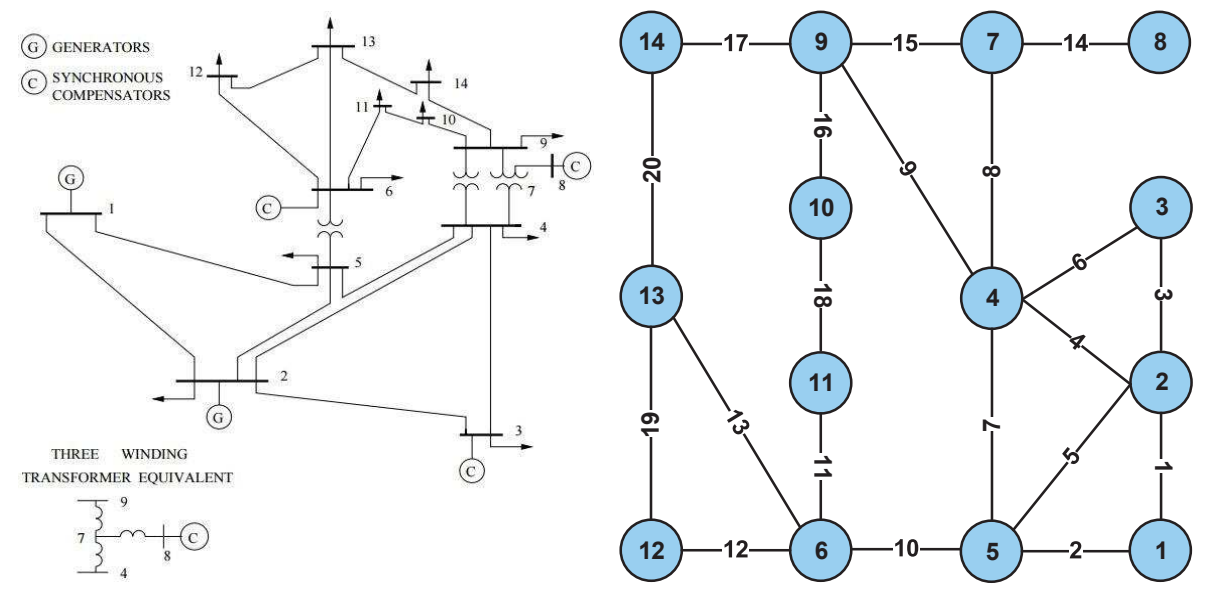

Figure 6.2: The IEEE 14-bus system contains 2 generators, 20 transmission lines. (a) Bus diagram. (b) Link Diagram.

illustrate the solutions of the game. The bus and line diagram of the system are given in Figure 6.2, including the total load of $259 \mathrm{MW}, 2$ generation buses, and 20 transmission lines. For simulating the game, the failures that disrupt only the transmission lines of the system are considered. Note that particular transmission lines in a power system are important, so that any failure on them will significantly cut off a large amount of power flow from generation to load. Thus, the operator (decision-maker) must react to these failures by restoring some links before the failures proceed to further stages.

Although the proposed framework can be applied to any finite number of states in power systems, only two power grids' states are defined in order to keep this simulation study numerically simple. If the total number of loads in the grid is $P_{\text {total }}$, two states $s_{1}$ and $s_{2}$ can be defined as $P_{\text {shed }}=0$ and $0<P_{\text {shed }} \leq P_{\text {total }}$, respectively. Also, it is assumed the limited budget $B_{f}=10$ for the failure maker to allocate it on the transmission lines in order to maximize the physical impact of the failures on the system. Similarly, the limited budget $B_{d}=10$ is assigned for the operator to distribute it on the transmission lines in order to maximize system survivability under multiple failures. The goal is to derive the Nash equilibrium strategies of both sides of the game for $s_{1}$ and $s_{2}$ based on the pre-assigned budgets. 
Table 6.1: Shed Load due to Failures in the IEEE 14-Bus System.

\begin{tabular}{llcclc}
\hline \hline $\begin{array}{l}\text { Failure } \\
\text { No. }\end{array}$ & Failure & $\begin{array}{c}\text { Shed Load } \\
(\mathrm{MW})\end{array}$ & $\begin{array}{c}\text { Failure } \\
\text { No. }\end{array}$ & $\begin{array}{l}\text { Failure } \\
\text { Location }\end{array}$ & $\begin{array}{c}\text { Shed Load } \\
(\mathrm{MW})\end{array}$ \\
\hline \hline $1\left(f_{1}\right)$ & Line 1 and 2 & 228 & $6\left(f_{3}\right)$ & Line 11 and 18 & 3.5 \\
2 & Line 1 and 5 & 0 & $7\left(f_{4}\right)$ & Line 12 and 19 & 6.1 \\
$3\left(f_{2}\right)$ & Line 3 and 6 & 94 & 8 & Line 16 and 17 & 0 \\
4 & Line 4 and 6 & 0 & $9\left(f_{5}\right)$ & Line 16 and 18 & 9 \\
5 & Line 11 and 13 & 0 & $10\left(f_{6}\right)$ & Line 12 and 20 & 14.9 \\
\hline \hline
\end{tabular}

In the 14-bus system, 10 types of two-failure events were investigated. The amount of shed load related to each of the successful failures is listed in Table 6.1, in which failure numbers $1,3,6$, and 8-10 are selected as the six severe multi-failure events that can cause more load to be shed than other failures. These critical multiple contingencies usually form the focus of the strategies for both sides of the game. Thus, the failure maker's action space can be formed by $\mathcal{F}=\left\{f_{1}, f_{2}, f_{3}, f_{4}, f_{5}, f_{6}\right\}$, as listed in Table 3.1. Similarly, the operator's response space $\mathcal{D}$ includes six corresponding response mechanisms, i.e., $\mathcal{D}=\left\{d_{1}, d_{2}, d_{3}, d_{4}, d_{5}, d_{6}\right\}$

Figure 6.3 shows the Nash equilibrium strategies for generating the multi-failure events and the corresponding operator's responses in states $s_{1}$ and $s_{2}$ for the 14-bus system with discount factors $\beta$ zero (static game), 0.5 , and 0.9 . The $y$-axis presents the probability, in which a certain failure or a response action can be chosen. As can be seen in Figure 6.3, the results show that the proposed game derives different Nash equilibrium strategies as the $\beta$ varies from 0 to 0.9 . For example, Figure 6.3(a) shows that in the state $s_{1}$ of the static game, the most severe failures can be generated by taking failure events $f_{1}$ and $f_{2}$, which would lead to more load to be shed when the failure succeeds. However, in the stochastic game, the best selections for generating the worth-case scenarios are failure events $f_{3}$ and $f_{4}$, in which less impacts are caused. This observation can be explained according to the operator's response strategy. In both the static game and the 


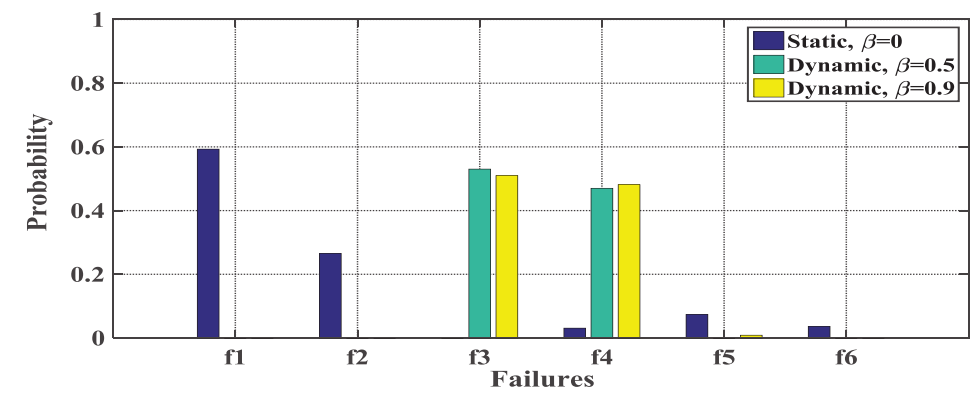

(a)

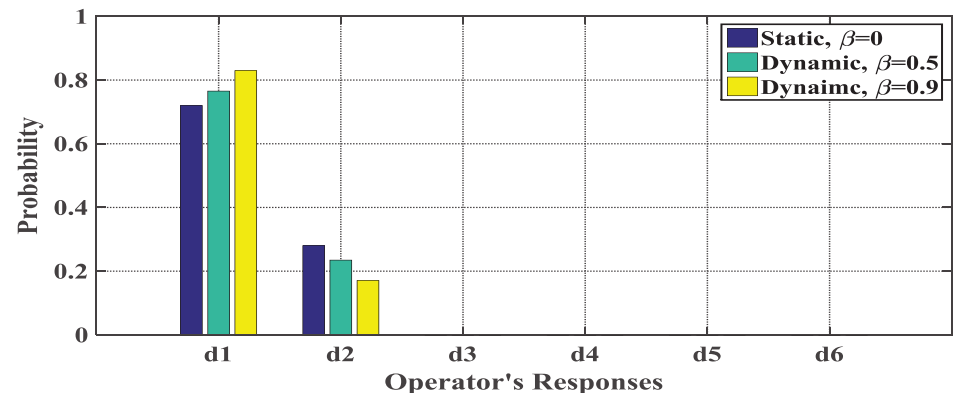

(b)

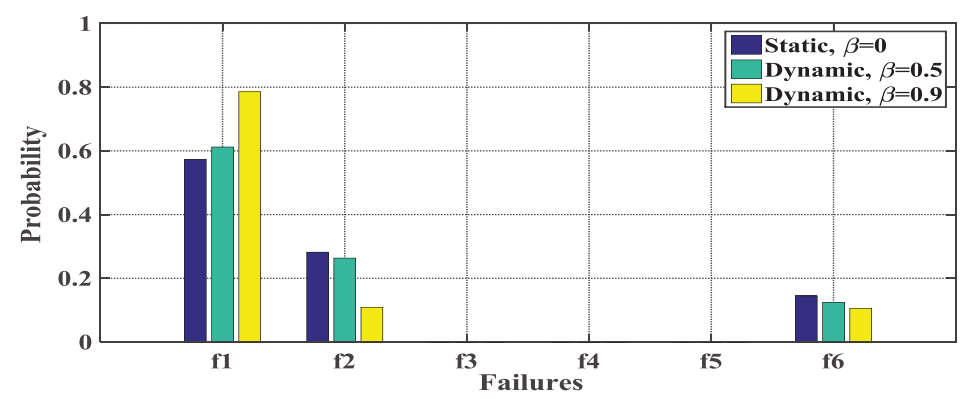

(c)

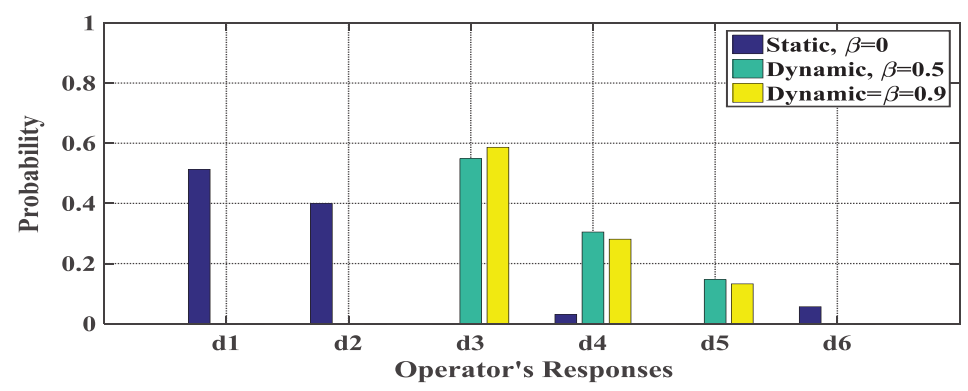

(d)

Figure 6.3: Nash equilibrium strategies with various discounted factors $\beta$ for IEEE 14bus system. (a) strategies of generating multi-failure events in state $s_{1}$. (b) operator's response strategies in state $s_{1}$. (c) strategies of generating severe multi-failure events in state $s_{2}$. (d) operator's response strategies in state $s_{2}$. 
stochastic game (see Figure 6.3(b)), the operator tries to survive the power grid, opting response actions $d_{1}$ and $d_{2}$ with adequately high probability. Although failure events $f_{1}$ and $f_{2}$ could lead to a higher physical damage, they are difficult to be successful under a proper survivability plan in the operator system. Therefore, the Nash equilibrium strategy for failure maker proposes the failure events with low probability of occurrence $\left(f_{3}\right.$ and $f_{4}$ ), which might cut off the transmission lines with less consideration from the power grid operator. Consequently in state $s_{2}$ of the stochastic game (Figures 6.3(c) and 6.(d)), the operator progressively changes its strategy by focusing more on response actions $d_{3}$, $d_{4}$ and $d_{5}$, as $\beta$ varies. This represents a continual interaction (in this effort 2 interactions) between the failure maker and power grid operator.

In addition to the IEEE 14-bus system, the IEEE 39-bus system, including the total load of $6100 \mathrm{MW}, 10$ generation buses, and 34 transmission lines, is also implemented as an additional benchmark for more comprehensive comparison, as shown in Figure 6.4. Similarly, 30 types of multi-failure events are investigated. The amount of shed load following each of the successful failures is listed in Table 6.2, in which failure numbers $3,5,8,10,11,15,24,25,28$ and 30 are chosen as ten severe multi-failure events with higher physical impacts on the system than others. Therefore, the failure maker's action space is formed by $\mathcal{F}=\left\{f_{1}, f_{2}, f_{3}, f_{4}, f_{5}, f_{6}, f_{7}, f_{8}, f_{9}, f_{10}\right\}$.

Given the same setting as the 14-bus system $\left(B_{f}=10\right.$ and $\left.B_{d}=10\right)$, Figure 6.5 presents the Nash equilibrium strategies for generating severe multi-failure events and the corresponding operator's responses in states $s_{1}$ and $s_{2}$ with various discount factors $\beta$. In $s_{1}$ of the stochastic game, the best selections for generating the severe failures are the $f_{3}$ with high probability and $f_{9}$ events, which make less physical impacts on the power systems, as shown in Figure 6.5(a) (i.e., based on the optimal load shedding in (5.1), Line 6, Line 24, Line 32, and Line 34 have less consideration from power grid operator). 


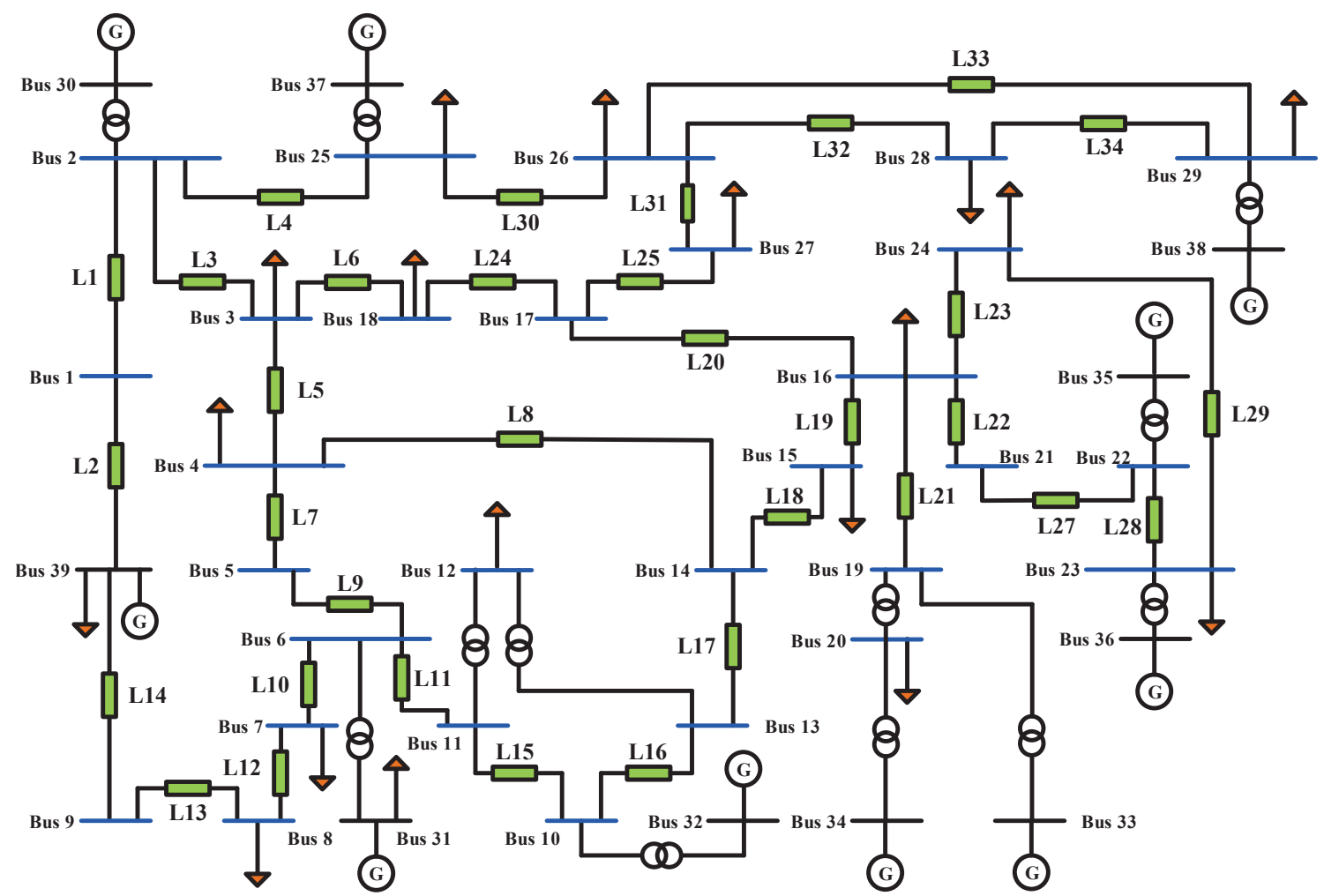

Figure 6.4: The IEEE 39-bus system contains 10 generators, 34 transmission lines, and 18 loads.

Figure 6.5(b) shows in state $s_{1}$ the operator's Nash equilibrium strategy varies less with different discount factors $\beta$ than the 14-bus system. Compared with the 14-bus system, the IEEE 39-bus system has a larger scale and the failure events might be less connected, so that the transmission lines selected to be failed are more independent between each other. Moreover, as state $s_{1}$ is the safe state without load to be shed, the operator's objective is just to reinforce the transmission lines against potential failures. Thus, the operator's Nash equilibrium strategy varies little between different game models. The continual interaction capability between the failure maker and power grid operator, which was the main goal of the proposed method, is represented in Figures 6.5(c) and 6.5(d).

Tables 6.3 and 6.4 show the Nash equilibrium strategies of the power system operator with various limited budgets $B_{d}$, namely 10, 20, 30, 40, and 50, assuming a limited 


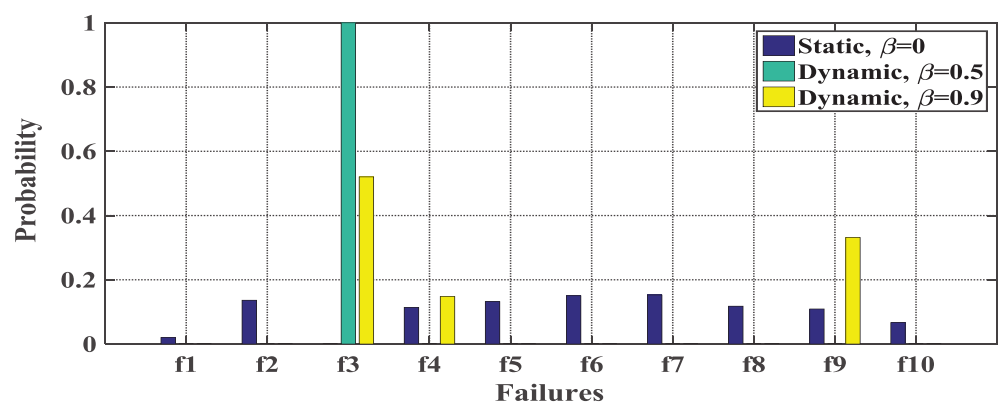

(a)

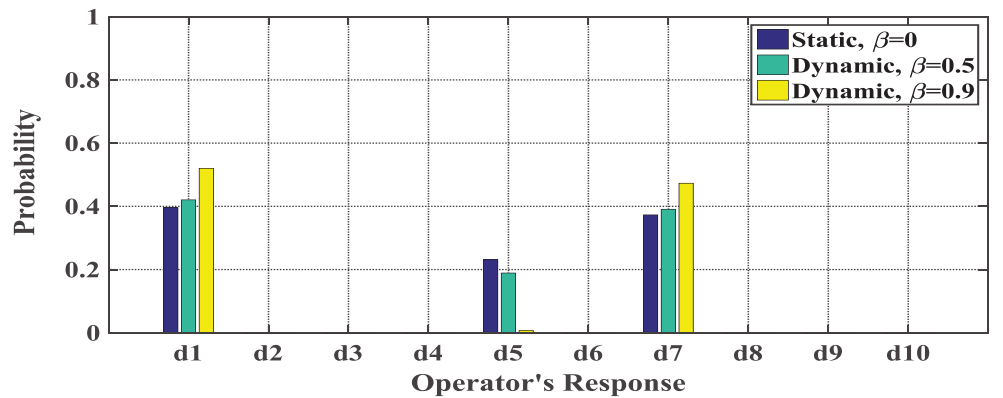

(b)

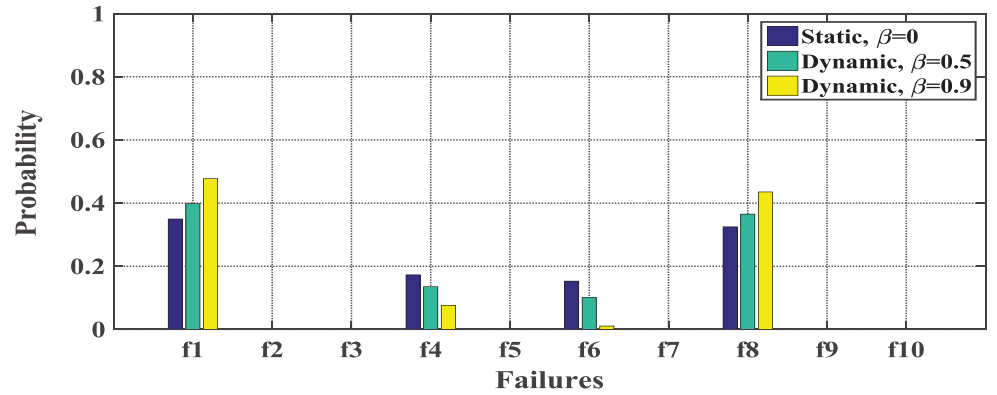

(c)

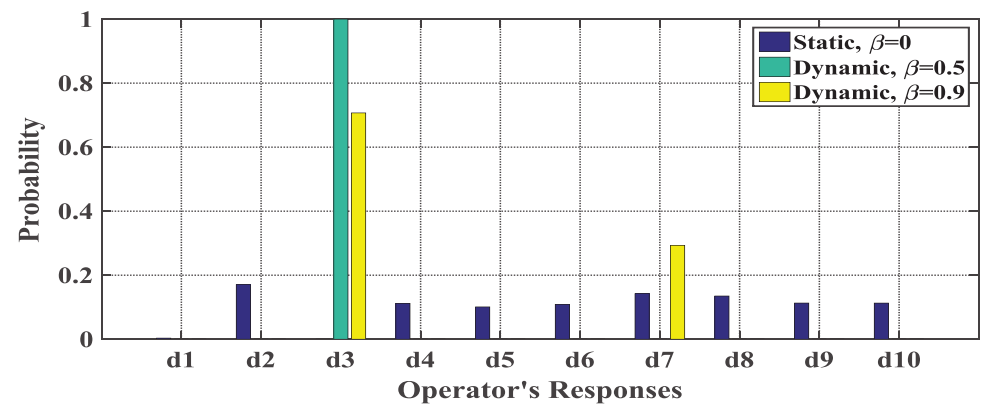

(d)

Figure 6.5: Nash equilibrium strategies with various discounted factors $\beta$ for the IEEE 39bus system. (a) severe generated multi-failure event strategies in state $s_{1}$. (b) operator's response strategies in state $s_{1}$. (c) severe generated multi-failure events strategies in state $s_{2}$. (d) operator's response strategies in state $s_{2}$. 
Table 6.2: Shed Load due to Failures in the IEEE 39-Bus System

\begin{tabular}{|c|c|c|c|c|c|}
\hline $\begin{array}{l}\text { Failure } \\
\text { No. }\end{array}$ & $\begin{array}{c}\text { Failure } \\
\text { Location }\end{array}$ & $\begin{array}{c}\text { Shed Load } \\
\text { (MW) }\end{array}$ & $\begin{array}{l}\text { Failure } \\
\text { No. }\end{array}$ & $\begin{array}{c}\text { Failure } \\
\text { Location }\end{array}$ & $\begin{array}{l}\text { Shed Load } \\
\text { (MW) }\end{array}$ \\
\hline 1 & Line 1 and 2 & 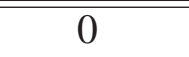 & 16 & Line 19 and 20 & 0 \\
\hline 2 & Line 1 and 3 & 0 & 17 & Line 20 and 24 & 0 \\
\hline $3\left(f_{1}\right)$ & Line 2 and 14 & 658 & 18 & Line 20 and 25 & 0 \\
\hline 4 & Line 3 and 6 & 0 & 19 & Line 22 and 23 & 0 \\
\hline $5\left(f_{2}\right)$ & Line 4 and 30 & 340 & 20 & Line 22 and 27 & 0 \\
\hline 6 & Line 5 and 6 & 0 & 21 & Line 23 and 25 & 0 \\
\hline 7 & Line 5 and 7 & 0 & 22 & Line 24 and 25 & 0 \\
\hline $8\left(f_{3}\right)$ & Line 6 and 24 & 158 & 23 & Line 25 and 26 & 0 \\
\hline 9 & Line 7 and 8 & 0 & $24\left(f_{7}\right)$ & Line 27 and 28 & 635 \\
\hline $10\left(f_{4}\right)$ & Line 10 and 12 & 233 & $25\left(f_{8}\right)$ & Line 28 and 29 & 333 \\
\hline $11\left(f_{5}\right)$ & Line 12 and 13 & 522 & 26 & Line 30 and 33 & 0 \\
\hline 12 & Line 13 and 14 & 0 & 27 & Line 31 and 32 & 0 \\
\hline 13 & Line 16 and 18 & 0 & $28\left(f_{9}\right)$ & Line 32 and 34 & 206 \\
\hline 14 & Line 15 and 17 & 0 & 29 & Line 33 and 30 & 0 \\
\hline $15\left(f_{6}\right)$ & Line 18 and 19 & 320 & $30\left(f_{10}\right)$ & Line 33 and 34 & 347 \\
\hline
\end{tabular}

Table 6.3: Nash Equilibrium Strategies of Operator for Various Budgets

\begin{tabular}{||l||l||}
\hline Budgets & \multicolumn{1}{|c||}{ Allocated Budgets for 14-Bus System } \\
\hline$B_{d}=10$ & $\mathrm{~S} 1:\left(L_{1}=L_{2}=3.83, L_{3}=L_{6}=1.17\right.$, and others are 0$)$ \\
& $\mathrm{S} 2:\left(L_{11}=2.35, L_{12}=L_{19}=1.85, L_{16}=0.8, L_{18}=3.15\right.$, others are 0$)$ \\
\hline$B_{d}=20$ & $\mathrm{~S} 1:\left(L_{1}=L_{2}=7.53, L_{3}=L_{6}=2.47\right.$, others are 0$)$ \\
& $\mathrm{S} 2:\left(L_{11}=4.82, L_{12}=L_{19}=3.78, L_{16}=1.4, L_{18}=6.22\right.$, others are 0$)$ \\
\hline$B_{d}=30$ & $\mathrm{~S} 1:\left(L_{1}=L_{2}=11.25, L_{3}=L_{6}=3.75\right.$, others are 0$)$ \\
& $\mathrm{S} 2:\left(L_{11}=6.94, L_{12}=L_{19}=4.61, L_{16}=3.45, L_{18}=10.39\right.$, others are 0$)$
\end{tabular}

budgets $B_{f}=10$ for failure maker and a given discount number $\beta=0.5$. The allocated budget for protecting the transmission lines of the IEEE 14-bus and 39-bus systems in states $s_{1}$ and $s_{2}$ are computed by multiplying the selection probability of a response action in each state by the assigned limited budget $B_{d}$.

As presented in Tables 6.3 and 6.4, for each protection budget, there is a Nash equi- 
Table 6.4: Nash Equilibrium Strategies of Operator for Various Budgets.

\begin{tabular}{|c|c|}
\hline Budgets & Allocated Budgets for 39-Bus System \\
\hline$B_{d}=10$ & $\begin{array}{l}\text { S1: }\left(L_{2}=L_{14}=2.1, L_{12}=L_{13}=0.94, L_{27}=L_{28}=1.95, \text { others are } 0\right) \\
\text { S2: }\left(L_{6}=L_{24}=5, \text { others are } 0\right)\end{array}$ \\
\hline$B_{d}=20$ & $\begin{array}{l}\text { S1: }\left(L_{2}=L_{14}=4.68, L_{12}=L_{13}=2.03, L_{27}=L_{28}=3.28 \text { others are } 0\right) \\
\text { S2: }\left(L_{6}=L_{24}=4.52, L_{32}=L_{34}=0.48, \text { others are } 0\right)\end{array}$ \\
\hline$B_{d}=30$ & $\begin{array}{l}\mathrm{S} 1:\left(L_{2}=L_{14}=8.36, L_{12}=L_{13}=1.91, L_{27}=L_{28}=4.75 \text { others are } 0\right) \\
\mathrm{S} 2:\left(L_{6}=L_{24}=13.14, L_{32}=L_{34}=1.86, \text { others are } 0\right)\end{array}$ \\
\hline$B_{d}=40$ & $\begin{array}{l}\mathrm{S} 1:\left(\mathrm{L}_{2}=\mathrm{L}_{14}=8.33 \mathrm{~L}_{12}=\mathrm{L}_{13}=3.89, \mathrm{~L}_{27}=\mathrm{L}_{28}=5.63, \mathrm{~L}_{33}=\mathrm{L}_{34}=2.13, \text { others are } 0\right) \\
\mathrm{S} 2:\left(\mathrm{L}_{6}=\mathrm{L}_{24}=14.12, \mathrm{~L}_{10}=\mathrm{L}_{12}=1.98, L_{32}=L_{34}=3.89, \text { others are } 0\right)\end{array}$ \\
\hline$B_{d}=50$ & $\begin{array}{l}\mathrm{S} 1:\left(\mathrm{L}_{2}=\mathrm{L}_{14}=8.42, \mathrm{~L}_{12}=\mathrm{L}_{13}=4.86, \mathrm{~L}_{27}=\mathrm{L}_{28}=9.04, \mathrm{~L}_{33}=\mathrm{L}_{34}=2.66, \text { others are } 0\right) \\
\mathrm{S} 2:\left(L_{6}=L_{24}=15.41, L_{10}=L_{12}=4.33, L_{32}=L_{34}=5.25, \text { others are } 0\right)\end{array}$ \\
\hline
\end{tabular}

librium strategy, so that it proposes the optimal allocation of limited budgets for some transmission lines in order to restore them before the failures proceed to further stages. For instance, in the 14-bus system, when 10, 20, or 30 budgets were implemented in state $s_{1}$, all of the budget, were allocated for lines $1,2,3$, and 6 , and any successful failures on them cause more load to be shed than other lines (see Table 6.3). When the limited budget increased to 50, some portion of the budget was allocated for lines 12 and 20, which have a risk of the shedding load in the 14-bus system. After a successful failure, resulting in shedding of partial loads (i.e., state $s_{2}$ ), the power system operator should allocate his/her limited budgets for the lines, such as lines $11,12,19$, and mostly lines 18 , which had less consideration in state $s_{1}$. In state $s_{1}$ and $s_{2}$ of the 39-bus system (Table 6.4), it can be clearly observed that by increasing the limited budget, more transmission lines would be under consideration (by assigning some portion of the budget), which lead to decrease of the amount of the shed loads, as well as risk of the outage.

Figure 6.6 presents the expected cost of shed load obtained from both the conventional Q-learning and modified Q-learning (by introducing the learning rate $\alpha_{t}$ ) for states $s_{1}$ and $s_{2}$ of the IEEE 14-bus and 39-bus system, assuming $B_{d}=B_{f}=10$ and $\beta=0.5$. As can be seen in Figure 6.6(a), the minimax Q-learning algorithm requires less iterations for con- 
verging to the Nash equilibrium compared with the proposed learning algorithm (around 30 iterations less than proposed game algorithm for $\omega=0.9$ ). However, the proposed algorithm yields lower expected costs of shed load for the operator. It is notable that, as $\omega$ increases, the modified algorithm reaches a lower expected costs of shed load due to the decrease of the learning rate $\alpha_{t}=1 /(t+1)^{\omega}$ proposed in the conventional method. In other words, the modified algorithm yields an expected cost reduction ranging between $5.336 \%$ (for $\omega=0.1$ ), $16 \%$ (for $\omega=0.5$ ), and $22.16 \%$ (for $\omega=0.9$ ) in state $s_{1}$ of the system relative to the minimax Q-learning algorithm. Compared to the IEEE 14-bus system, the modified algorithm for the IEEE 39-bus system results in a higher cost of load shedding with the increase of the $\omega$. For example, the proposed algorithm with $\omega=0.8$ converges to an expected cost $356 \$ / \mathrm{kW}$, which is 6 times the cost of $\omega=0.9$ in state $s_{1}$ of the 14-bus system.

\subsection{Summary}

This chapter has presented a new approach based on stochastic game theory to study survivability of the power grid under severe concurrent multi-failure events. In this study, the multiple failures have been generated by the failure maker introduced as one side of the game-theoretic framework. An optimal load shedding approach is proposed to calculate the minimum cost of shed load under different types of failures by generating multiple failures with the low and high probability of occurrence. The complex interactions between the failure maker and power grid operator have been formulated by considering the limited budgets introduced in the stochastic game. Also, this chapter has proposed a modified Q-learning algorithm to find the Nash equilibrium strategies of this game with a less cost of load shedding, compared with the conventional Q-learning, in order to provide realistic guidelines on how to deploy limited budgets for protecting critical components of the power grid. The IEEE 14-bus and 39-bus systems are used as test models to illustrate 


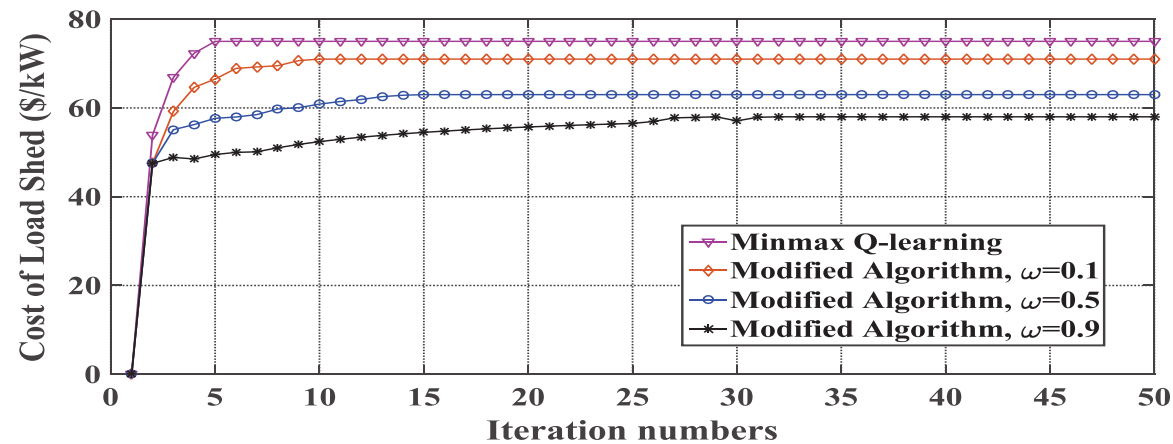

(a)

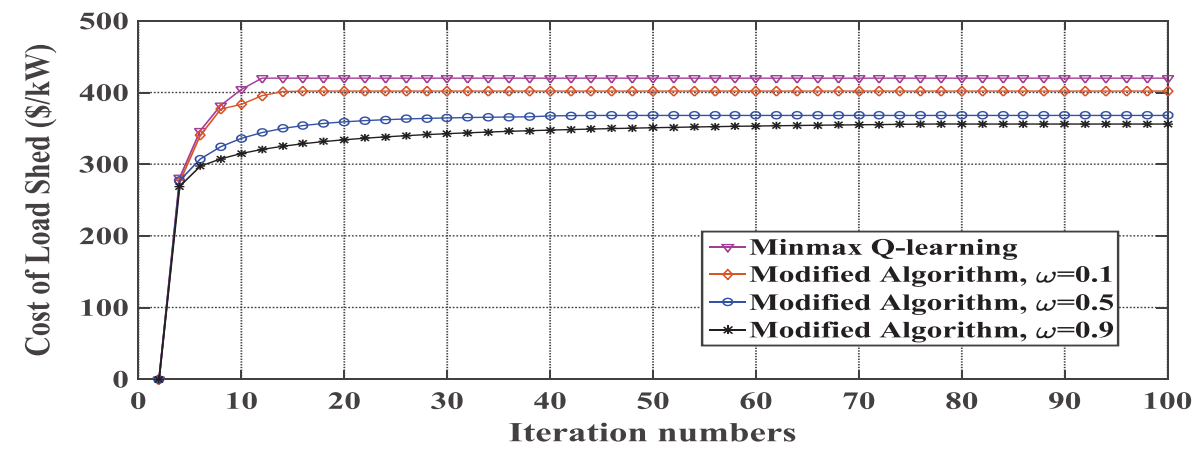

(b)

Figure 6.6: Comparison of expected cost of load shedding in state $s_{1}$ of the stochastic game, (a) IEEE 14-bus system. (b) IEEE 39-bus system. 
solutions of the proposed game. Simulation results have shown that the proposed game method can provide the optimal allocation of limited budgets for some transmission lines in order to restore them before the failures proceed to further stages. 


\section{CHAPTER 7}

\section{CONCLUSION AND FUTURE WORK}

\subsection{Conclusion}

The integration of renewable energy resources (RESs) with improved penetration has been of keen interest to the engineers and power system operators for a while now. Research institutes are still conducting studies to assess the possibility of increasing the penetration of RES. However, grid integration of large RESs particularly in weak grid can pose the adverse effects on in several significant ways, such as transient and voltage stability, existing protection schemes, power leveling and energy balancing. To protect the network from threats related to these issues, this dissertation has developed the advanced and algorithmic methods for specification of the protection scheme, reactive power capability and power control requirements for interconnection of the PV and wind power plants into the smart grid. In this dissertation, different aspects related to the design, optimization, implementation, protection, and energy management of smart grid systems with renewable energy sources are presented.

Wind turbines with the grid-connected mode of the operation play a significant role toward in sustainable energy development in the future, in which a non-negligible $20 \%$ of them is still employing the FSIGs. Thus, the fault-ride through characteristics of FSIGbased wind turbines still need to be analyzed. In chapter 2, the comprehensive review of the state-of-the-art developments for LVRT capability improvement of WTs based on FSIGs is given. All reviewed methodologies have been categorized into three main groups, i.e., series-connected solutions, shunt-connected solutions, and hybrid-connected solutions; discussing the performance of the LVRT schemes including their advantages and limitations in the detail. Also, an analysis of these LVRT methods in terms of control complexity and economic feasibility has been comparatively investigated and summa-

rized in Table 2.1, so that it concludes that the overall cost and control complexity of the 
SFCL and UPQC schemes are higher than other types of LVRT technologies. On the other hand, the SDBR and BFCL methods were relatively the cheapest and simplest control structure among other LVRT solutions from the economic feasibility point of view. For verification purposes, several simulations has been presented in MATLAB software to compare the reviewed LVRT schemes. Based on the simulated results, series connection DVR and shunt connection STATCOM are the highly efficient LVRT capability enhancement approaches. Thus, this study has provided the guidelines for researchers in order to choose a suitable technique for the LVRT capability improvement of WT systems.

Application of superconducting devices, such as resistive SFCL (RSFCL) and SMES for optimal performance of the WTs has been proposed in chapter 3. The main efforts are categorized into three sections. The application of the RSFCL, in providing an additional low voltage ride-through support to the wind-driven FSIG, has been investigated in chapter3-section 3.1. RSFCL can limit the grid current during the fault and minimize the voltage reduction at the generator terminal, leading to a compliance with international grid codes. The results has been demonstrated that the integration of the FSIG-based WT will become more promising from the energy-saving and downsizing perspective by introducing the RSFCL as self-healing limiter. Furthermore, the application of the RSFCL, in providing an additional low voltage ride-through support to the PMSG-based WT, has been studied in chapter3-section 3.2. it has been demonstrated that the RSFCL can be a promising solution for improving the WT controller performance with respect to the dclink fluctuations and the extreme loads on the wind. The proposed RSFCL can decrease the activation of the dc braking chopper and fast pitch angle control systems, thereby increasing the reliability of the system. A further study has been performed to determine the optimal performance of the combinatorial 50 MW PMSG-WPP and RSFCL using a multiobjective optimization based on AHP. 
A micro-SMES system integrated to the VSWT-PMSG, which is interconnected to the standalone dc microgrid through the bi-directional DC-DC converter, has been proposed in chapter3-section 3.3. A power balance between loads, micro-SMES system and nondeterministic wind generation has been maintained via presenting the supervisory control architecture. For this purpose, an optimal design scheme based on SA algorithm has been modeled for micro-SMES solenoid coil to ensure the desired energy storage capacity (880 kJ) with least volume. Moreover, this section presents an effective control strategy to obtain a rapid response of the micro-SMES system to the wind speed fluctuations in order to stabilize dc-link voltage; thereby smoothing the output power simultaneously. This penetrative approach allows finding the optimized charging and discharging model based on the exponential function.

Chapter 4 has presented a computational intelligence technique based on a multiobjective optimization model in order to design an external interface controller for the coordinated reactive power control between the DFIG-based WT and the STATCOM, during the faults. Two conflict objectives were voltage deviations during and after grid faults and low-frequency oscillations of the active power after clearing the faults. This chapter has introduced a new index called transient power severity index (TPSI) to quantify the transient active power performance of the system. This proposed methodology provides a Pareto front for decision makers to determine the optimal tradeoff between these two objectives. For online applications, the optimal commanded values of the compensating reactive power for both RSC of the DFIG and the STATCOM controllers have been achieved via an FLC, which is properly tuned using the NSA algorithm. Simulation results have shown that the proposed control approach can successfully improve the LVRT capability of the WT in the weak grid-connected mode. Moreover, it can operate as an external damping controller for the WT, and therefore, improve the post-fault power oscillation damping of system. 
Regarding the design and hardware implementation, the 300-VA three-phase singlestage CSI, with dc voltage boost capability, has been proposed and has been experimentally implemented as a testbed for grid-tied PV MIC systems. The switching pattern based on the phasor pulse-width-modulated (PPWM) technique has been employed to produce the switching signals through regulating the modulation index $m_{i}$ and modulation angle $\alpha_{0}$. The state-space averaging method, in combination with the $d q$ reference frame, has been used to derive the large- and small-signal models in order to study the dynamic behaviors of the CSI-based MIC system. Surprisingly, it is concluded that the dc-link voltage has never affected the stability of the proposed system. The results also reveal that the proposed system stays stable as control parameters of $m_{i}$ and $\alpha_{0}$ vary within the CSI's operating limits. The multivariable PI-based current control strategy with structural simplicity has been presented to independently control the active and reactive powers. The experimental results has showed that the proposed control system has a promising dynamic response in terms of tracking the step changes in the active and reactive power reference values. Furthermore, the multivariable PI control method represents a superior axis decoupling capability, so that the step changes in one power command signal negligibly affect the other.

Chapter 6 has developed a new approach based on the framework of stochastic game theory to enable a power system to recover from severe and concurrent failures, before such failures turn into a full blown cascading failure. In this study, the multiple failures have been generated by the failure maker introduced as one side of the game-theoretic framework. First, an optimal load shedding technique has been introduced to quantify the physical impact of worst-case contingencies by generating simultaneously multi-failure events at various points of the power network. Given these quantified impacts as input parameters, continual interactions between the failure maker, which generates severe 
multi-failure events, and the power grid operator (decision maker) have been formulated as a stochastic game. In this game, the goal of failure makers was to increase the probability of successful multiple failures and the goal of power grid operators was to increase the probability of successful response mechanisms. The complex interactions between the failure maker and power grid operator have been formulated by considering the limited budgets introduced in stochastic game. To find the Nash equilibrium of this game, a new learning algorithm based on the modified Q-learning algorithm has been proposed with a less cost of load shedding compared to the conventional Q-learning method. Simulation results for two IEEE standard 14-bus and 39-Bus systems have shown that the proposed game method can provide the optimal allocation of limited budgets for some transmission lines in order to restore them, before the failures proceed to further stages.

\subsection{Future Work}

The topics covered in this dissertation illustrated the new challenges in real-time control and optimal operation of smart grid system with integration of large renewable energy sources. Due to essential needs for understanding, recognizing and solving probable issues and obstacles of future power systems, it recommended to consider the following topics as the future works:

1. As demonstrated in chapter 3, the length of the recovery period of the SFCL is a function of the superconducting characteristics used for modeling of the SFCL. As part of future work, comprehensive studies will be suggested to determine an accurate model for the SFCL in order to obtain acceptable recovery periods for the potential applications, especially in RES integrations. Moreover, the comparison of various kind of the SFCL technologies, such as the resistive SFCL, the shield-core SFCL, and the saturable-core SFCL, saturated type SFCL would be an interesting research in this field of area. 
2. There are several interesting future research areas for this field study presented in chapter 4. For example in this dissertation, multiple DFIGs were experimented with one WT, in both modeling and simulation. Although this is a justifiable simplification due to the fact that there are normally no mutual interactions between wind turbines on a wind farm, it would be worth to take into consideration more multiple DFIG models, which are close to real cases, to prove the feasibility of the proposed control method, and to assist a realistic grid-connected wind farm development. In addition to the proposed power network as presented in this thesis, it would be interesting to model and analyze relatively large and complex power system scenarios to demonstrate the effectiveness of the proposed coordinated reactive power control for such a complex system. Finally, the validity of the proposed approach can be further verified by the experimental results of a small-scaled DFIG and STATCOM prototype.

3. Considering further expansion of the three-phase PV MIC system, it would be required for MIC to be equipped with the real-time active and reactive power control capability to fulfill the upcoming grid requirements. Thus, the investigations in chapter 5 can pave the path for the CSI-based MIC applications into photovoltaic energy generation systems. This chapter has also presented the tools to further explore the CSI-based MIC capabilities for other case studies, such as analyzing stability of the closed-loop control systems and coordinating the output power of the high number of the CSI-based MIC units as a large-scale grid-tied PV systems. Different faults of the system should can be studied, and correspondingly proper protection and control systems can be designed for the CSI.

4. The interactions between the failure maker and power grid operator are formed as different games in chapter 6. However, a large power system needs to take much time to implement the algorithms. Although a pruning strategy is proposed, it is still 
primitive. Therefore, further developing the framework and enhancing the efficiency of the proposed algorithms will be our future work. Also, the smart grid system is more complicated than the model used in this dissertation because it consists of the power network and the communication network interconnected by edges and occurrence of failures in one network triggers failures in the other network. 


\section{BIBLIOGRAPHY}

[1] T. Mai, R. Wiser, D. Sandor, and G. Brinkman, "Renewable electricity futures study volume 1: Exploration of high-penetration renewable electricity futures," NREL, 2012.

[2] J. Enslin, "Grid impacts and solutions of renewables at high penetration levels," QUANTA Technology, 2009.

[3] M. Mohseni and S. M. Islam, "Review of international grid codes for wind power integration: Diversity, technology and a case for global," Renewable and Sustainable Energy Reviews, vol. 16, no. 1, pp. 3876-3890, 2012.

[4] M. Tsili and S. Papathanassiou, "A review of grid code technical requirements for wind farms," IET Renewable Power Generation, vol. 3, no. 3, pp. 308-332, 2009.

[5] "Grid connection of wind turbines to networks with voltages below $100 \mathrm{kv}$," Regulation TF 3.2.6, Energinet, Frederica, Denmark, 2004.

[6] S. M. Muyeen, J. Tamura, and T. Murata, Stability Augmentation of a Gridconnected Wind Farm. Springer, 2008.

[7] O. Anaya-Lara, N. Jenkins, J. Ekanayake, P. Cartwright, and M. Hughes, Wind energy generation modeling and control. Wiley, 2009.

[8] A. Moghadasi and A. Islam, "Enhancing LVRT capability of FSIG wind turbine using current source UPQC based on resistive SFCL," TD Conference and Exposition, 2014 IEEE PES, pp. 1-5, 2014.

[9] A. H. Moghadasi, A. Islam, and M. Amini, "LVRT capability assessment of FSIGbased wind turbine utilizing UPQC and SFCL," PES General Meeting, Conference Exposition, 2014 IEEE, pp. 1-5, 2014.

[10] Z. Zhang, Y. Zhao, W. Qiao, and L. Qu, "A discrete-time direct torque control for direct-drive PMSG-based wind energy conversion systems," IEEE Transactions on Industry Applications, vol. 51, no. 4, pp. 3504-3514, 2015.

[11] K. H. Kim, Y. C. Jeung, D. C. Lee, and H. G. Kim, "LVRT scheme of PMSG wind power systems based on feedback linearization," IEEE Transactions on Power Electronics, vol. 27, no. 5, pp. 2376-2384, 2012. 
[12] V. Yaramasu, B. Wu, S. Alepuz, and S. Kouro, "Predictive control for low-voltage ride-through enhancement of three-level-boost and NPC-converter-based PMSG wind turbine," IEEE Transactions on Industrial Electronics, vol. 61, no. 12, pp. 6832-6843, 2014.

[13] W. Qiao, G. Venayagamoorthy, and R. Harley, "Real-time implementation of a STATCOM on a wind farm equipped with doubly fed induction generators," IEEE Transactions on Industry Applications, vol. 45, no. 1, pp. 98-107, 2009.

[14] Y. Tang, P. Ju, H. He, C. Qin, and F. Wu, "Optimized control of DFIG-based wind generation using sensitivity analysis and particle swarm optimization," IEEE Transactions on Smart Grid, vol. 4, no. 1, pp. 509-520, 2013.

[15] W. Qiao, R. G. Harley, and G. K. Venayagamoorthy, "Coordinated reactive power control of a large wind farm and a STATCOM using heuristic dynamic programming," IEEE Transactions on Energy Conversion, vol. 24, no. 2, pp. 493-503, 2009.

[16] H. Gaztanaga, I. Etxeberria-Otadui, D. Ocnasu, and S. Bacha, "Real-time analysis of the transient response improvement of fixed-speed wind farms by using a reduced-scale STATCOM prototype," IEEE Transactions on Power Systems, vol. 22, no. 2, pp. 658-666, 2007.

[17] R. Singh, G. Alapatt, and A. Lakhtakia, "Making solar cells a reality in every home: Opportunities and challenges for photovoltaic device design," Electron Devices Society, IEEE Journal of the, vol. 1, no. 6, pp. 129-144, 2013.

[18] S. A. Khajehoddin, M. Karimi-Ghartemani, A. Bakhshai, and P. Jain, "A power control method with simple structure and fast dynamic response for single-phase grid-connected DG systems," IEEE Transactions on Power Electronics, vol. 28, no. 1, pp. 221-233, 2013.

[19] Q. Li and P. Wolfs, "A review of the single phase photovoltaic module integrated converter topologies with three different DC link configurations," Power Electronics, IEEE Transactions on, vol. 23, no. 3, pp. 1320-1333, 2008.

[20] M. Noe and B. R. Oswald, "Technical and economical benefits of superconducting fault current limiters in power systems," IEEE Transactions on Applied Superconductivity, vol. 9, no. 2, pp. 1347-1350, 1999. 
[21] H. Heydari and A. H. Moghadasi, "Optimization scheme in combinatorial UPQC and SFCL using normalized simulated annealing," IEEE Transactions on Power Delivery, vol. 26, no. 3, pp. 1489-1498, 2011.

[22] S. Eckroad, "Superconducting fault current limiters," Electric Power Research Institute (EPRI) Transactions on Power Delivery, 2008.

[23] Y. Du, S. Baek, S. Bhattacharya, and A. Q. Huang, "High-voltage high-frequency transformer design for a $7.2 \mathrm{kv}$ to $120 \mathrm{v} / 240 \mathrm{v} 20 \mathrm{kva}$ solid state transformer," in IECON 2010 - 36th Annual Conference on IEEE Industrial Electronics Society, 2010, pp. 493-498.

[24] Y. Zhu, J. Yan, Y. Sun, and H. He, "Revealing cascading failure vulnerability in power grids using risk-graph," IEEE Transactions on Parallel and Distributed Systems, vol. 25, no. 12, pp. 3274-3284, 2014.

[25] "The economic impacts of the august 2003 blackout," The Electricity Consumers Resource Council (ELCON), 2004.

[26] "High-impact, low-frequency event risk to the north american bulk power system," North American Electric Reliability Corporation (NERC), 2010.

[27] I. Erlich, W. Winter, and A. Dittrich, "Advanced grid requirements for the integration of wind turbines into the german transmission system," in Power Engineering Society General Meeting, 2006. IEEE, 2006, p. 7.

[28] S. Sheng, K. K. Li, W. L. Chan, Z. Xiangjun, and D. Xianzhong, "Agent-based self-healing protection system," IEEE Transactions on Power Delivery, vol. 21, no. 2, pp. 610-618, 2006.

[29] D. Dustegor, T. E. Mezyani, and S. K. Srivastava, "A distributed fault protection method for power grid with high penetration of renewable energy sources," in $S$ mart Grid Communications (SmartGridComm), 2011 IEEE International Conference on, 2011, pp. 564-569.

[30] L. Ye and L. Z. Lin, "Study of superconducting fault current limiters for system integration of wind farms," IEEE Transactions on Applied Superconductivity, vol. 20, no. 3, pp. 1233-1237, 2010.

[31] S. Muyeen, M. Ali, T. Murata, and J. Tamura, “Transient stability enhancement of wind generator by a new logical pitch controller," IEEE Transactions on Power Energy, vol. 126, p. 742752, 2006. 
[32] M. J. Hossain, H. R. Pota, V. A. Ugrinovskii, and R. A. Ramos, "Simultaneous STATCOM and pitch angle control for improved LVRT capability of fixed-speed wind turbines," IEEE Transactions on Sustainable Energy, vol. 1, no. 3, pp. 142$151,2010$.

[33] T. Kinjo, T. Senjyu, N. Urasaki, and H. Fujita, “Terminal-voltage and output-power regulation of wind-turbine generator by series and parallel compensation using SMES," IEE Proceedings - Generation, Transmission and Distribution, vol. 153, no. 3, pp. 276-282, 2006.

[34] A. Moghadasi, A. Sarwat, and J. M. Guerrero, "A comprehensive review of lowvoltage-ride-through methods for fixed-speed wind power generations," Renewable and Sustainable Energy reviews, Elsevier, vol. 55, pp. 823-839, 2016.

[35] R. A. Ibrahim, M. S. Hamad, Y. G. Dessouky, and B. W. Williams, "A novel topology for enhancing the low voltage ride through capability for grid connected wind turbine generators," in Energy Conversion Congress and Exposition (ECCE), 2012 IEEE, 2012, pp. 2389-2395.

[36] Y. Gui, C. Kim, and C. C. Chung, "Nonlinear control for pmsg wind turbine via port-controlled hamiltonian system," in PowerTech, 2015 IEEE Eindhoven, 2015, pp. 1-6.

[37] A. Mullane, G. Lightbody, and R. Yacamini, "Wind-turbine fault ride-through enhancement," IEEE Transactions on Power Systems, vol. 20, no. 4, pp. 1929-1937, 2005.

[38] K. Amei, Y. Takayasu, T. Ohji, and M. Sakui, "A maximum power control of wind generator system using a permanent magnet synchronous generator and a boost chopper circuit," in Power Conversion Conference, 2002. PCC-Osaka 2002. Proceedings of the, vol. 3, 2002, pp. 1447-1452 vol.3.

[39] M. Farhadi and O. Mohammed, "Adaptive energy management in redundant hybrid DC microgrid for pulse load mitigation," IEEE Transactions on Smart Grid, vol. 6, no. 1, pp. 54-62, 2015.

[40] F. Deng and Z. Chen, "Adaptive energy management in redundant hybrid DC microgrid for pulse load mitigation," in Proc. IEEE IECON, , p. 621626, 2009.

[41] J. Matas, M. Castilla, J. M. Guerrero, L. G. de Vicuna, and J. Miret, "Feedback linearization of direct-drive synchronous wind-turbines via a sliding mode approach," IEEE Transactions on Power Electronics, vol. 23, no. 3, pp. 1093-1103, 2008. 
[42] L. Xu and D. Chen, "Control and operation of a DC microgrid with variable generation and energy storage," IEEE Transactions on Power Delivery, vol. 26, no. 4, pp. 2513-2522, 2011.

[43] A. Moghadasi, H. Heydari, and M. Farhadi, "Pareto optimality for the design of SMES solenoid coils verified by magnetic field analysis," IEEE Transactions on Applied Superconductivity, vol. 21, no. 1, pp. 13-20, 2011.

[44] S. Nomura, Y. Ohata, T. Hagita, H. Tsutsui, S. Tsuji-Iio, and R. Shimada, "Wind farms linked by SMES systems," IEEE Transactions on Applied Superconductivity, vol. 15, no. 2, pp. 1951-1954, 2005.

[45] H. Louie and K. Strunz, "Superconducting magnetic energy storage (SMES) for energy cache control in modular distributed hydrogen-electric energy systems," IEEE Transactions on Applied Superconductivity, vol. 17, no. 2, pp. 2361-2364, 2007.

[46] H. Kakigano, Y. Miura, and T. Ise, "Low-voltage bipolar-type DC microgrid for super high quality distribution," IEEE Transactions on Power Electronics, vol. 25, no. 12, pp. 3066-3075, 2010.

[47] "http://www.superpower-inc.com/content/superconducting-magnetic-energystorage-smes."

[48] Y. Mishra, S. Mishra, and F. Li, "Coordinated tuning of DFIG-based wind turbines and batteries using bacteria foraging technique for maintaining constant grid power output," IEEE Systems Journal, vol. 6, no. 1, pp. 16-26, 2012.

[49] F. M. Gonzalez-Longatt, P. Wall, P. Regulski, and V. Terzija, "Optimal electric network design for a large offshore wind farm based on a modified genetic algorithm approach," IEEE Systems Journal, vol. 6, no. 1, pp. 164-172, 2012.

[50] F. Wu, X. P. Zhang, K. Godfrey, and P. Ju, "Small signal stability analysis and optimal control of a wind turbine with doubly fed induction generator," IET Generation, Transmission Distribution, vol. 1, no. 5, pp. 751-760, 2007.

[51] T. D. Vrionis, X. I. Koutiva, and N. A. Vovos, "A genetic algorithm-based low voltage ride-through control strategy for grid connected doubly fed induction wind generators," IEEE Transactions on Power Systems, vol. 29, no. 3, pp. 1325-1334, 2014. 
[52] G. Venayagamoorthy, "Dynamic, stochastic, computational, and scalable technologies for smart grids," IEEE Computational Intelligence Magazine, vol. 6, no. 3, pp. 22-35, 2011.

[53] Y. Tanga, H. Hea, Z. Nia, J. Wenb, and X. Suib, "Dynamic, stochastic, computational, and scalable technologies for smart grids," Neurocomputing, vol. 125, pp. 125-133, 2014.

[54] E. Aggelogiannaki and H. Sarimveis, "A simulated annealing algorithm for prioritized multiobjective optimization mdash;implementation in an adaptive model predictive control configuration," IEEE Transactions on Systems, Man, and Cybernetics, Part B (Cybernetics), vol. 37, no. 4, pp. 902-915, 2007.

[55] E. Aggeloginnaki and H. Sarimveis, "Optimization by simulated annealing," Science, vol. 220, pp. 671-680, 1983.

[56] Y. Zhou, L. Liu, and H. Li, "A high-performance photovoltaic module-integrated converter (MIC) based on cascaded quasi-z-source inverters (qZSI) using egan FETs," IEEE Transactions on Power Electronics, vol. 28, no. 6, pp. 2727-2738, 2013.

[57] B. Mirafzal, M. Saghaleini, and A. K. Kaviani, "An svpwm-based switching pattern for stand-alone and grid-connected three-phase single-stage boost inverters," IEEE Transactions on Power Electronics, vol. 26, no. 4, pp. 1102-1111, 2011.

[58] F. Z. Peng, "Z-source inverter," IEEE Transactions on Industry Applications, vol. 39, no. 2, pp. 504-510, 2003.

[59] Y. Chen and K. Smedley, "Three-phase boost-type grid-connected inverter," IEEE Transactions on Power Electronics, vol. 23, no. 5, pp. 2301-2309, 2008.

[60] B. Sahan, A. N. Vergara, N. Henze, A. Engler, and P. Zacharias, "A single-stage pv module integrated converter based on a low-power current-source inverter," IEEE Transactions on Industrial Electronics, vol. 55, no. 7, pp. 2602-2609, 2008.

[61] D. N. Zmood and D. G. Holmes, "Improved voltage regulation for current source inverters," in Industry Applications Conference, 2000. Conference Record of the 2000 IEEE, vol. 4, 2000, pp. 2353-2360 vol.4.

[62] M. Salo and H. Tuusa, "A vector controlled current-source pwm rectifier with a novel current damping method," IEEE Transactions on Power Electronics, vol. 15, no. 3, pp. 464-470, 2000. 
[63] L. Chen, A. Amirahmadi, Q. Zhang, N. Kutkut, and I. Batarseh, "Design and implementation of three-phase two-stage grid-connected module integrated converter," IEEE Transactions on Power Electronics, vol. 29, no. 8, pp. 3881-3892, 2014.

[64] C. Schauder and H. Mehta, "Vector analysis and control of advanced static var compensators," IEE Proceedings C - Generation, Transmission and Distribution, vol. 140, no. 4, pp. 299-306, 1993.

[65] F. Blaabjerg, R. Teodorescu, M. Liserre, and A. V. Timbus, "Overview of control and grid synchronization for distributed power generation systems," IEEE Transactions on Industrial Electronics, vol. 53, no. 5, pp. 1398-1409, 2006.

[66] M. Liserre, R. Teodorescu, and F. Blaabjerg, "Multiple harmonics control for threephase grid converter systems with the use of PI-RES current controller in a rotating frame," IEEE Transactions on Power Electronics, vol. 21, no. 3, pp. 836-841, 2006.

[67] M. Castilla, J. Miret, J. Matas, L. G. de Vicuna, and J. M. Guerrero, "Control design guidelines for single-phase grid-connected photovoltaic inverters with damped resonant harmonic compensators," IEEE Transactions on Industrial Electronics, vol. 56, no. 11, pp. 4492-4501, 2009.

[68] B. Bahrani, S. Kenzelmann, and A. Rufer, "Multivariable-PI-based dq current control of voltage source converters with superior axis decoupling capability," IEEE Transactions on Industrial Electronics, vol. 58, no. 7, pp. 3016-3026, 2011.

[69] V. Krishnan, "Machine learning aided efficient tools for risk evaluation and operational planning of multiple contingencies," Springer International Publishing Switzerland, 2015.

[70] J. Kim, J. A. Bucklew, and I. Dobson, "Splitting method for speedy simulation of cascading blackouts," in Power and Energy Society General Meeting (PES), 2013 IEEE, 2013, pp. 1-1.

[71] A. Chegu and F. Li, "High order contingency selection based on particle swarm optimization and branch reordering," in IPEC, 2010 Conference Proceedings, 2010, pp. 1111-1116.

[72] B. O. Mkandawire, N. M. Ijumba, and H. Whitehead, "Asset management optimization through integrated systems thinking and N-1 contingency capability for refurbishment," IEEE Systems Journal, vol. 5, no. 3, pp. 321-331, 2011. 
[73] B. C. Lesieutre, S. Roy, V. Donde, and A. Pinar, "Power system extreme event screening using graph partitioning," in Power Symposium, 2006. NAPS 2006. 38th North American, 2006, pp. 503-510.

[74] L. Mili and K. Dooley, "Risk-based power system planning integrating social and economic direct and indirect costs," in John Wiley, vol. 2, 2008, pp. 503-510.

[75] W. Saad, Z. Han, H. V. Poor, and T. Basar, "Game-theoretic methods for the smart grid: An overview of microgrid systems, demand-side management, and smart grid communications," IEEE Signal Processing Magazine, vol. 29, no. 5, pp. 86-105, 2012.

[76] C. Ma, D. Yau, X. Lou, and N. Rao, "Markov game analysis for attack-defense of power networks under possible misinformation," IEEE Transactions on Power Systems, vol. 28, no. 2, pp. 1676-1686, 2013.

[77] Y. W. Law, T. Alpcan, and M. Palaniswami, "Security games for risk minimization in automatic generation control," IEEE Transactions on Power Systems, vol. 30, no. 1, pp. 223-232, 2015.

[78] M. Anghel, K. A. Werley, and A. E. Motter, "Stochastic model for power grid dynamics," in System Sciences, 2007. HICSS 2007. 40th Annual Hawaii International Conference on, 2007, pp. 113-113.

[79] L. Holdsworth, X. G. Wu, J. B. Ekanayake, and N. Jenkins, "Comparison of fixed speed and doubly-fed induction wind turbines during power system disturbances," IEE Proceedings - Generation, Transmission and Distribution, vol. 150, no. 3, pp. 343-352, 2003.

[80] F. Blaabjerg, Z. Chen, and S. B. Kjaer, "Power electronics as efficient interface in dispersed power generation systems," IEEE Transactions on Power Electronics, vol. 19, no. 5, pp. 1184-1194, 2004.

[81] Z. Chen, J. M. Guerrero, and F. Blaabjerg, "A review of the state of the art of power electronics for wind turbines," IEEE Transactions on Power Electronics, vol. 24, no. 8, pp. 1859-1875, 2009.

[82] M. Sathyajith, "Wind energy: fundamentals, resource analysis and economics," Springer Science and Business Media, 2006. 
[83] V. Akhmatova, H. Knudsena, A. H. Nielsenb, J. K. Pedersenb, and N. K. Poulsen, "Modelling and transient stability of large wind farms," International Journal of Electrical Power and Energy Systems, vol. 25, pp. 123-144, 2003.

[84] F. Bianchi, R. Mantz, and C. Christiansen, "Gain scheduling control of variablespeed wind energy conversion systems using quasi-LPV models," Control Engineering Practice, vol. 13, pp. 247-255, 2005.

[85] R. Rocha and L. S. M. Filho, "A multivariable h; control for wind energy conversion system," in Control Applications, 2003. CCA 2003. Proceedings of 2003 IEEE Conference on, vol. 1, 2003, pp. 206-211.

[86] J. Slootweg, S. D. Haan, H. Polinder, and W. Kling, "General model for representing variable speed wind turbines in power system dynamics simulations," IEEE Transactions on Power Systems, vol. 18, no. 1, pp. 144-151, 2003.

[87] R. Chedid, F. Mrad, and M. Basma, "Intelligent control of a class of wind energy conversion systems," IEEE Transactions on Energy Conversion, vol. 14, no. 4, pp. 1597-1604, 1999.

[88] A. Sumper, O. Gomis-Bellmunt, A. Sudria-Andreu, R. Villafafila-Robles, and J. Rull-Duran, "Response of fixed speed wind turbines to system frequency disturbances," IEEE Transactions on Power Systems, vol. 24, no. 1, pp. 181-192, 2009.

[89] R. C. Bansal, "Three-phase self-excited induction generators: an overview," IEEE Transactions on Energy Conversion, vol. 20, no. 2, pp. 292-299, 2005.

[90] J. Dixon, L. Moran, J. Rodriguez, and R. Domke, "Reactive power compensation technologies: State-of-the-art review," Proceedings of the IEEE, vol. 93, no. 12, pp. 2144-2164, 2005.

[91] E. Muljadi and C. P. Butterfield, "Pitch-controlled variable-speed wind turbine generation," IEEE Transactions on Industry Applications, vol. 37, no. 1, pp. 240-246, 2001.

[92] R. Piwko, N. Miller, J. Sanchez-Gasca, X. Yuan, R. Dai, and J. Lyons, "Integrating large wind farms into weak power grids with long transmission lines," in Transmission and Distribution Conference and Exhibition: Asia and Pacific, 2005 IEEE/PES, 2005, pp. 1-7. 
[93] O. Gomis-Bellmunt, A. Junyent-Ferr, A. Sumper, and J. Bergas-Jan, "Ride-through control of a doubly fed induction generator under unbalanced voltage sags," IEEE Transactions on Energy Conversion, vol. 23, no. 4, pp. 1036-1045, 2008.

[94] E. Muljadi, D. Yildirim, T. Batan, and C. P. Butterfield, "Understanding the unbalanced-voltage problem in wind turbine generation," in Industry Applications Conference, 1999. Thirty-Fourth IAS Annual Meeting. Conference Record of the 1999 IEEE, vol. 2, 1999, pp. 1359-1365.

[95] C. Wessels, N. Hoffmann, M. Molinas, and F. W. Fuchs, "StatCom control at wind farms with fixed-speed induction generators under asymmetrical grid faults," IEEE Transactions on Industrial Electronics, vol. 60, no. 7, pp. 2864-2873, 2013.

[96] C. Wessels, F. W. Fuchs, and M. Molinas, "Voltage control of a statcom at a fixed speed wind farm under unbalanced grid faults," in IECON 2011 - 37th Annual Conference on IEEE Industrial Electronics Society, 2011, pp. 979-984.

[97] B. Singh, S. S. Murthy, and S. Gupta, "STATCOM-based voltage regulator for self-excited induction generator feeding nonlinear loads," IEEE Transactions on Industrial Electronics, vol. 53, no. 5, pp. 1437-1452, 2006.

[98] G. Huang and T. Zhu, "TCSC as a transient voltage stabilizing controller," in Power Engineering Society Winter Meeting, 2001. IEEE, vol. 2, 2001, pp. 628-633 vol.2.

[99] A. D. Rosso, C. Canizares, and V. Dona, "A study of TCSC controller design for power system stability improvement," IEEE Transactions on Power Systems, vol. 18, no. 4, pp. 1487-1496, 2003.

[100] P. Mattavelli, G. C. Verghese, and A. M. Stankovic, "Phasor dynamics of thyristorcontrolled series capacitor systems," IEEE Transactions on Power Systems, vol. 12, no. 3, pp. 1259-1267, 1997.

[101] J. J. Paserba, "How FACTS controllers benefit ac transmission systems," in Power Engineering Society General Meeting, 2004. IEEE, 2004, pp. 1257-1262 Vol.2.

[102] H. Zhou, H. Wei, X. Qiu, J. Xu, X. Wei, and S. Wang, "Improvement of transient voltage stability of the wind farm using SVC and TCSC," in Power and Energy Engineering Conference (APPEEC), 2011 Asia-Pacific, 2011, pp. 1-4.

[103] N. N. Joshi and N. Mohan, "Application of TCSC in wind farm application," in Power Electronics, Electrical Drives, Automation and Motion, 2006. SPEEDAM 2006. International Symposium on, 2006, pp. 1196-1200. 
[104] E. V. Larsen, K. Clark, S. A. Miske, and J. Urbanek, "Characteristics and rating considerations of thyristor controlled series compensation," IEEE Transactions on Power Delivery, vol. 9, no. 2, pp. 992-1000, 1994.

[105] H. Gaztanaga, I. Etxeberria-Otadui, S. Bacha, and D. Roye, "Fixed-speed wind farm operation improvement by using DVR devices," in Industrial Electronics, 2007. ISIE 2007. IEEE International Symposium on, 2007, pp. 2679-2684.

[106] D. Ramirez, S. Martinez, C. A. Platero, F. Blazquez, and R. M. de Castro, "Lowvoltage ride-through capability for wind generators based on dynamic voltage restorers," IEEE Transactions on Energy Conversion, vol. 26, no. 1, pp. 195-203, 2011.

[107] C. Meyer, R. W. D. Doncker, Y. W. Li, and F. Blaabjerg, “Optimized control strategy for a medium-voltage DVR: Theoretical investigations and experimental results," IEEE Transactions on Power Electronics, vol. 23, no. 6, pp. 2746-2754, 2008.

[108] N. Joshi and N. Mohan, "A novel scheme to connect wind turbines to the power grid," IEEE Transactions on Energy Conversion, vol. 24, no. 2, pp. 504-510, 2009.

[109] Y. W. Li, D. M. Vilathgamuwa, F. Blaabjerg, and P. C. Loh, "A robust control scheme for medium-voltage-level DVR implementation," IEEE Transactions on Industrial Electronics, vol. 54, no. 4, pp. 2249-2261, 2007.

[110] A. Causebrook, D. J. Atkinson, and A. G. Jack, "Fault ride-through of large wind farms using series dynamic braking resistors (march 2007)," IEEE Transactions on Power Systems, vol. 22, no. 3, pp. 966-975, 2007.

[111] W. Freitas, A. Morelato, and W. Xu, "Improvement of induction generator stability using braking resistors," IEEE Transactions on Power Systems, vol. 19, no. 2, pp. 1247-1249, 2004.

[112] J. A. Wiik, F. D. Widjaya, T. Isobe, T. Kitahara, and R. Shimada, "Series connected power flow control using magnetic energy recovery switch (MERS)," in Power Conversion Conference - Nagoya, 2007. PCC '07, 2007, pp. 983-990.

[113] J. A. Wiik, O. J. Fnstelien, and R. Shimada, "A MERS type series FACTS controller for low voltage ride through of induction generators in wind farms," in IEEE Conference, 20010, pp. 1-10. 
[114] J. A. Wiik, F. D. Wijaya, and R. Shimada, "An innovative series connected power flow controller, magnetic energy recovery switch (MERS)," in Power Engineering Society General Meeting, 2007. IEEE, 2007, pp. 1-7.

[115] J. Wiik, F. Wijaya, and R. Shimada, "Characteristics of the magnetic energy recovery switch (MERS) as a series FACTS controller," IEEE Transactions on Power Delivery, vol. 24, no. 2, pp. 828-836, 2009.

[116] A. R. Fereidouni, B. Vahidi, and T. H. Mehr, "The impact of solid state fault current limiter on power network with wind-turbine power generation," IEEE Transactions on Smart Grid, vol. 4, no. 2, pp. 1188-1196, 2013.

[117] H. G. Sarmiento, R. Castellanos, G. Pampin, C. Tovar, and J. Naude, "An example in controlling short circuit levels in a large metropolitan area," in Power Engineering Society General Meeting, 2003, IEEE, vol. 2, 2003, p. 594 Vol. 2.

[118] M. Firouzi and G. B. Gharehpetian, "Improving fault ride-through capability of fixed-speed wind turbine by using bridge-type fault current limiter," IEEE Transactions on Energy Conversion, vol. 28, no. 2, pp. 361-369, 2013.

[119] M. Jafari, S. B. Naderi, M. T. Hagh, M. Abapour, and S. H. Hosseini, "Voltage sag compensation of point of common coupling (PCC) using fault current limiter," IEEE Transactions on Power Delivery, vol. 26, no. 4, pp. 2638-2646, 2011.

[120] G. Rashid and M. H. Ali, "A modified bridge-type fault current limiter for fault ride-through capacity enhancement of fixed speed wind generator," IEEE Transactions on Energy Conversion, vol. 29, no. 2, pp. 527-534, 2014.

[121] Y. Salami and M. Firouzi, "Dynamic performance of wind farms with bridgetype superconducting fault current limiter in distribution grid," in Electric Power and Energy Conversion Systems (EPECS), 2011 2nd International Conference on, 2011, pp. 1-6.

[122] M. T. Hagh, K. M. Muttaqi, D. Sutanto, M. S. A. Hossain, and A. M. A. Haidar, "Improving fault ride-through capability of DFIG based wind generators by using bridge-type superconducting fault current limiter," in Power Engineering Conference (UPEC), 2015 50th International Universities, 2015, pp. 1-6.

[123] M. H. Haque, "Improvement of first swing stability limit by utilizing full benefit of shunt FACTS devices," in IEEE TRANSACTIONS ON POWER SYSTEMS, vol. 19, no. 4, 2004, pp. 1894-1902. 
[124] M. Haque, "Thyristor-based FACTS controllers for electrical transmission systems," in IEEE Press and Wiley, New York, 2002.

[125] M. Narimani and R. K. Varma, "Application of static var compensator (SVC) with fuzzy controller for grid integration of wind farm," in Electrical and Computer Engineering (CCECE), 2010 23rd Canadian Conference on, 2010, pp. 1-6.

[126] S. H. Huang, J. Schmall, J. Conto, J. Adams, Y. Zhang, and C. Carter, "Voltage control challenges on weak grids with high penetration of wind generation: ERCOT experience," in Power and Energy Society General Meeting, 2012 IEEE, 2012, pp. $1-7$.

[127] L. Xu, L. Yao, and C. Sasse, "Comparison of using SVC and STATCOM for wind farm integration," in Power System Technology, 2006. PowerCon 2006. International Conference on, 2006, pp. 1-7.

[128] M. Molinas, J. A. Suul, and T. Undeland, "Low voltage ride through of wind farms with cage generators: STATCOM versus SVC," IEEE Transactions on Power Electronics, vol. 23, no. 3, pp. 1104-1117, 2008.

[129] R. J. Koessler, "Dynamic simulation of static VAr compensators in distribution systems," in Transmission and Distribution Conference, 1991., Proceedings of the 1991 IEEE Power Engineering Society, 1991, pp. 273-279.

[130] M. Noroozian, N. A. Petersson, B. Thorvaldson, A. B. Nilsson, and C. W. Taylor, "Benefits of SVC and STATCOM for electric utility application," in Transmission and Distribution Conference and Exposition, 2003 IEEE PES, vol. 3, 2003, pp. 1143-1150 vol.3.

[131] S. Muyeena, R. Takahashia, T. Murataa, J. Tamuraa, and M. H. Ali, "Application of statcom/bess for wind power smoothening and hydrogen generation," in Electric Power Systems Research, vol. 79, 2009, pp. 365-373.

[132] J. A. Suul, M. Molinas, and T. Undeland, "STATCOM-based indirect torque control of induction machines during voltage recovery after grid faults," IEEE Transactions on Power Electronics, vol. 25, no. 5, 2010.

[133] H. MJ., P. HR., and R. RA., "Robust STATCOM control for the stabilization of fixed-speed wind turbines during low voltages," Renewable Energy, vol. 36, no. 11, 2011. 
[134] L. Zhou, J. Liu, and F. Liu, "Design and implementation of STATCOM combined with series dynamic breaking resistor for low voltage ride-through of wind farms," in Energy Conversion Congress and Exposition (ECCE), 2010 IEEE, 2010, pp. 2501-2506.

[135] M. Hossain, H. Pota, and R. Ramos, "Improved low-voltage-ride-through capability of fixed-speed wind turbines using decentralised control of STATCOM with energy storage system," in IET Generation, Transmission and Distribution,, vol. 6, no. 8, 2012.

[136] D. T. Bradshaw, "'super" reactive power for the power system through supervar/spl trade/ high temperature superconductor dynamic synchronous condensers," in Power Engineering Society General Meeting, 2004. IEEE, 2004, pp. 2058-2061.

[137] M. Ross and S. Kalsi, "Applications of superconducting synchronous condenser$\mathrm{s}$ in wind power integration," in Transmission and Distribution Conference and Exhibition, 2005/2006 IEEE PES, 2006, pp. 272-277.

[138] S. Teleke, T. Abdulahovic, T. Thiringer, and J. Svensson, "Dynamic performance comparison of synchronous condenser and SVC," IEEE Transactions on Power Delivery, vol. 23, no. 3, pp. 1606-1612, 2008.

[139] N. G. Jayanti, M. Basu, M. F. Conlon, and K. Gaughan, "Rating requirements of the unified power quality conditioner to integrate the fixedspeed induction generatortype wind generation to the grid," IET Renewable Power Generation, vol. 3, no. 2, pp. 133-143, 2009.

[140] A. Moghadasi, H. Heydari, and M. Salehifar, "Reduction in VA rating of the unified power quality conditioner with superconducting fault current limiters," in Power Electronic Drive Systems Technologies Conference (PEDSTC), 2010 1st, 2010, pp. 382-387.

[141] P. Huang, M. Moursi, W. Xiao, and J. Kirtley, "Fault ride-through configuration and transient management scheme for self-excited induction generator-based wind turbine," IEEE Transactions on Sustainable Energy, vol. 5, no. 1, pp. 148-159, 2014.

[142] B. Singh, "Introduction to FACTS controllers in wind power farms: A technological review," International Journal of Renewable Energy Research-IJRER, vol. 2, no. 2, pp. 1-47, 2012. 
[143] M. Saravanan, S. M. R. Slochanal, P. Venkatesh, and J. P. Abraham, "Application of particle swarm optimization technique for optimal location of FACTS devices considering cost of installation and system loadability," Electric Power Systems Research, vol. 77, no. 3, pp. 276-283, 2007.

[144] Y. Zhang, "Techno-economic assessment of voltage sag performance and mitigation," PhD Thesis, University of Manchester, 2008.

[145] M. Brito, M. Cavalcanti, L. Limongiu, and F. Neves, "Low cost dynamic voltage restorer," International Conference on Renewable Energies and Power Quality (ICREPQ12), 2012.

[146] "World dynamic voltage restorer (DVR) markets," Frost and Sullivan Research Service, 2005.

[147] W. Gong, Y. Wang, S. Hu, and H. Xu, "A survey on recent low voltage ride-through solutions of large scale wind farm," in Power and Energy Engineering Conference (APPEEC), 2011 Asia-Pacific, 2011, pp. 1-5.

[148] F. Fran, K. John, T. Rizy, and T. King, "A preliminary analysis of the economics of using distributed energy as a source of reactive power supply," in Department of Energy (DOE), 2006.

[149] H. M. Chang, G. H. Lee, J. Sim, K. B. Park, I. S. Oh, J. B. Song, and H. Lee, "Two-stage cryocooling design for hybrid superconducting fault current limiter," IEEE Transactions on Applied Superconductivity, vol. 20, no. 3, pp. 2047-2050, 2010.

[150] E. F. Pleva and S. W. Schwenterly, "Assembly and test of 5/10 MVA HTS transformer," in Power Engineering Society General Meeting, 2004. IEEE, 2004, pp. 1431-1435.

[151] S. Heier, "Grid integration of wind energy conversion systems," in ohn Wiley and Sons, 1998.

[152] L. Fernndeza, C. Garcaa, J. Saenza, and F. Juradob, "Equivalent models of wind farms by using aggregated wind turbines and equivalent winds," Energy Conversion and Management, vol. 50, no. 3, pp. 691-704, 2009.

[153] E. S. Abdin and W. Xu, "Control design and dynamic performance analysis of a wind turbine-induction generator unit," IEEE Transactions on Energy Conversion, vol. 15, no. 1, pp. 91-96, 2000. 
[154] B. Gromoll, G. Ries, W. Schmidt, H.-P. Kramer, P. Kummeth, and H.-W. Neumuller, "Resistive current limiters with YBCO films s," IEEE Transactions on Applied Superconductivity, vol. 7, no. 2, pp. 828-831, 1997.

[155] D. K. Park, M. C. Ahn, S. E. Yang, Y. S. Yoon, B. Y. Seok, C. Lee, H. M. Chang, and T. K. Ko, "Development of $220 \mathrm{v} / 300$ a class non-inductive winding type fault current limiter using 2g HTS wire," IEEE Transactions on Applied Superconductivity, vol. 17, no. 2, pp. 1863-1866, 2007.

[156] H. Shimizu, Y. Yokomizu, M. Goto, T. Matsumura, and N. Murayama, "A study on required volume of superconducting element for flux flow resistance type fault current limiter," IEEE Transactions on Applied Superconductivity, vol. 13, no. 2, pp. 2052-2055, 2003.

[157] S. Y. Kim, W. W. Kim, and J. O. Kim, "Determining the location of superconducting fault current limiter considering distribution reliability," IET Generation, Transmission Distribution, vol. 6, no. 3, pp. 240-246, 2012.

[158] J. Adhikari, I. V. Prasanna, and S. K. Panda, "Maximum power-point tracking of high altitude wind power generating system using optimal vector control technique," in Power Electronics and Drive Systems (PEDS), 2015 IEEE 11th International Conference on, 2015, pp. 773-778.

[159] A. Gyore, S. Semperger, V. Tihanyi, I. Vajda, M. R. Gonal, K. P. Muthe, S. C. Kashyap, and D. K. Pandya, "Experimental analysis of different type HTS rings in fault current limiter," IEEE Transactions on Applied Superconductivity, vol. 17, no. 2, pp. 1899-1902, 2007.

[160] H. Heydari and R. Sharifi, "Three-dimensional pareto-optimal design of inductive superconducting fault current limiters," IEEE Transactions on Applied Superconductivity, vol. 20, no. 5, pp. 2301-2311, 2010.

[161] P. F. Ribeiro, B. K. Johnson, M. L. Crow, A. Arsoy, and Y. Liu, "Energy storage systems for advanced power applications," Proceedings of the IEEE, vol. 89, no. 12, pp. 1744-1756, 2001.

[162] G. Zhu, M. Song, Z. Wang, and S. Dai, "Design of LTS coil used for the combined device of SMES-SFCL," IEEE Transactions on Applied Superconductivity, vol. 16, no. 2, pp. 674-677, 2006. 
[163] M. H. Amini, B. Nabi, and M. R. Haghifam, "Load management using multiagent systems in smart distribution network," in Power and Energy Society General Meeting (PES), 2013 IEEE, 2013, pp. 1-5.

[164] M. H. Amini, B. Nabi, M. P. Moghaddam, and S. A. Mortazavi, "Evaluating the effect of demand response programs and fuel cost on PHEV owners behavior, a mathematical approach," in Smart Grids (ICSG), 2012 2nd Iranian Conference on, 2012, pp. 1-6.

[165] S. M. Blair, C. D. Booth, N. K. Singh, G. M. Burt, and C. G. Bright, "Analysis of energy dissipation in resistive superconducting fault-current limiters for optimal power system performance," IEEE Transactions on Applied Superconductivity, vol. 21, no. 4, pp. 3452-3457, 2011.

[166] Y. Xu, Z. Y. Dong, K. Meng, W. F. Yao, R. Zhang, and K. P. Wong, "Multiobjective dynamic VAR planning against short-term voltage instability using a decomposition-based evolutionary algorithm," IEEE Transactions on Power Systems, vol. 29, no. 6, pp. 2813-2822, 2014.

[167] J. M. Garibaldi and E. C. Ifeachor, "Application of simulated annealing fuzzy model tuning to umbilical cord acid-base interpretation," IEEE Transactions on Fuzzy Systems, vol. 7, no. 1, pp. 72-84, 1999.

[168] S. Jiang, U. D. Annakkage, and A. M. Gole, "A platform for validation of FACTS models," IEEE Transactions on Power Delivery, vol. 21, no. 1, pp. 484-491, 2006.

[169] A. Singh, A. Kaviani, and B. Mirafzal, "On dynamic models and stability analysis of three-phase phasor PWM-based CSI for stand-alone applications," Industrial Electronics, IEEE Transactions on, vol. 62, no. 5, pp. 2698-2707, 2015.

[170] B. Mirafzal, M. Saghaleini, and A. Kaviani, "An SVPWM-based switching pattern for stand-alone and grid-connected three-phase single-stage boost inverters," Power Electronics, IEEE Transactions on, vol. 26, no. 4, pp. 1102-1111, 2011.

[171] J. A. Sanders and F. Verhulst, "Averaging methods in nonlinear dynamical systems," New York, Springer-Verlag, 1985.

[172] A. K. Kaviani, "Dynamic modeling and analysis of single-stage boost inverters under normal and abnormal conditions," PhD Dissertation, Florida International University, 2012. 
[173] J. D. Bastidas-Rodriguez, E. Franco, G. Petrone, C. A. Ramos-Paja, and G. Spagnuolo, "Maximum power point tracking architectures for photovoltaic systems in mismatching conditions: a review," IET Power Electronics, vol. 7, no. 6, pp. 1396-1413, 2014.

[174] B. Sahan, A. Vergara, N. Henze, A. Engler, and P. Zacharias, "A single-stage pv module integrated converter based on a low-power current-source inverter," Industrial Electronics, IEEE Transactions on, vol. 55, no. 7, pp. 2602-2609, 2008.

[175] A. Pinar, J. Meza, V. Donde, and B. Lessieutre, "Optimization strategies for the vulnerability analysis of the electric power grid," SIAM J. Optimiz, vol. 20, no. 4, pp. 1786-1810, 2010.

[176] J. Nocedal and S. Wright, “Numerical optimization,” Springer Press, 2006.

[177] J. Nocedal and S. Wrighty, "Convex optimization," Cambridge, U.K.: Cambridge Univ. Press, 2004.

[178] A. Neyman and S. Sorin, "Stochastic games and applications," New York: Kluwer Academic, 1999.

[179] Y. Shoham and K. Leyton-Brown, "Multiagent systems: Algorithmic, gametheoretic, and logical foundations," Cambridge, U.K.: Cambridge Univ. Press, 2009.

[180] E. Alpaydin, “Introduction to machine learning," MIT Press, 2012.

[181] J. Yan, Y. Tang, H. He, and Y. Sun, "Cascading failure analysis with DC power flow model and transient stability analysis," IEEE Transactions on Power Systems, vol. 30, no. 1, pp. 285-297, 2015. 
VITA

\section{AMIRHASAN MOGHADASIRISEH}

June 14, 1984

2001-2004

2004-2008

2008-2010

2013-2016

2016
Born, Tehran, Iran

National Diploma, Mathematics and Physics Alborz High School

Tehran, Iran

B.S., Electrical Engineering Shahed University

Tehran, Iran

M.S., Electrical Engineering Iran University of science and technology Tehran, Iran

Doctoral Candidate, Electrical Engineering Florida International University

Miami, Florida

Dissertation Evidence Acquisition Fellowship Florida International University

\section{PUBLICATIONS AND PRESENTATIONS}

[1] A. Moghadasi, A. Sarwat, J. M. Guerrero, "A Comprehensive Review of Low-VoltageRide-Through Methods for Fixed-Speed Wind Power Generations," Renewable and Sustainable Energy Reviews, Elsevier., vol.55, No.1, pp. 823-839, March 2016.

[2] A. Moghadasi, A. Sarwat, J. M. Guerrero, "Multiobjective Optimization in Combinatorial Wind Farms System Integration and Resistive SFCL Using Analytical Hierarchy Process," Renewable Energy, Elsevier., vol.136, No.1, pp. 173-180, March 2016

[3] M. Moghaddami, A. Moghadasi and A. I. Sarwat "An Algorithm for Fast Calculation of Short Circuit Forces in High Current Busbars of EAF Transformers Based on Method of Images," Electric Power System Research, Elsevier., vol.136, pp. 173-180, 2016.

[4] A. Moghadasi, A. Sargolzaei, A. I. Sarwat, Akshay K. Rathore and K. Yen, "A Simplified Power Control Approach with Reliable Axis Decoupling Capability for ThreePhase Current Source Inverter.," IEEE Transaction on Power Electronic., Under Review, Manuscript ID.: TPEL-Reg-2016-03-0461. 
[5] A. Moghadasi, M. Moghaddami, A. Anzalchi, A. I. Sarwat, and Osama A. Mohammed, "Prioritized Coordinated Reactive Power Control of Wind Turbine Involving STATCOM Using Multi-Objective Optimization," IEEE Transaction on Industrial Application., Under Review, Manuscript ID.: 2016-ESC-0075.

[6] A. Anzalchi, A. Moghadasi, M. Moghaddami, A. I. Sarwat, and M. Akshay, "A New Topology of Higher Order Power Filter for Single-Phase Grid-tied Voltage Source Inverters," IEEE Transaction on Industrial Electronics., Under Review (Revised), Manuscript No.: 15-TIE-3933.

[7] A. Anzalchi, M. Moghaddami, A. Moghadasi, Maneli Malek Pour, and A. I. Sarwat, "A Modified Higher Order Power Filter for Grid-Connected Renewable Energy Systems," IEEE Transaction on Industrial Application., Under Review, Manuscript ID.: 2016-ESC0069

[8] A. Moghadasi, A. I. Sarwat, H. Amini, "LVRT capability assessment of FSIG-based wind turbine utilizing UPQC and SFCL," PES General Meeting Conference and Exposition., 2014 IEEE, National Harbor, MD, 2014, pp. 1-5.

[9] A. Moghadasi and A. Islam, "Enhancing LVRT capability of FSIG wind turbine using current source UPQC based on resistive SFCL," T and D Conference and Exposition., 2014 IEEE PES, Chicago, IL, USA, 2014, pp. 1-5.

[10] A. Moghadasi and A. I. Sarwat, "Optimal analysis of resistive superconducting fault current limiters applied to a variable speed wind turbine system," SoutheastCon 2015. Fort Lauderdale, FL, 2015, pp. 1-7.

[11] A. Moghadasi, A. Sundararajan and A. I. Sarwat, "Power Management and Control Strategy in Standalone DC Microgrid along with SMES Solenoid Coil," Journal of Enhanced Research in Science Technology and Engineering., Vol. 3 Issue 10, October-2014, pp 102-112.

[12] A. Moghadasi, A. Sargolzaei, A. Sarwat, K. Yen, "Active and Reactive Power Control Method for Three-Phase PV Module-Integrated Converter Based on a Single-Stage Inverter," Accepted in IEEE International Symposium on Industrial Electronics (ISIE 2016) Santa Clara, CA, June 08-10, 2016.

[13] A. Sargolzaei, A. Moghadasi, K. Yen and A. I. Sarwat, "Time-delay analysis on grid-connected three-phase current source inverter based on SVPWM switching pattern," Computational Intelligence Applications in Smart Grid (CIASG)., 2014 IEEE Symposium on, Orlando, FL, 2014, pp. 1-5.

[14] M. Moghaddami, A. Moghadasi, A. I. Sarwat, "A Single-Stage Three-Phase AC-AC Converter for Inductive Power Transfer Systems," Accepted to 2016 IEEE Power and Energy Society General Meeting (PES-GM).

[15] A. I. Sarwat, A. Domijan, M. H. Amini, and A. Moghadasi, "Smart Grid reliability assessment utilizing Boolean Driven Markov Process and variable weather conditions," North American Power Symposium (NAPS)., Charlotte, NC, 2015, pp. 1-6. 